\title{
How cardiac myofibers keep pace : mathematical modeling of adaptive myofiber reorientation and electromechanics
}

Citation for published version (APA):

Pluijmert, M. H. (2015). How cardiac myofibers keep pace : mathematical modeling of adaptive myofiber reorientation and electromechanics. [Doctoral Thesis, Maastricht University]. Maastricht University. https://doi.org/10.26481/dis.20150924mp

Document status and date:

Published: 01/01/2015

DOI:

10.26481/dis.20150924mp

Document Version:

Publisher's PDF, also known as Version of record

Please check the document version of this publication:

- A submitted manuscript is the version of the article upon submission and before peer-review. There can be important differences between the submitted version and the official published version of record.

People interested in the research are advised to contact the author for the final version of the publication, or visit the DOI to the publisher's website.

- The final author version and the galley proof are versions of the publication after peer review.

- The final published version features the final layout of the paper including the volume, issue and page numbers.

Link to publication

\footnotetext{
General rights rights.

- You may freely distribute the URL identifying the publication in the public portal. please follow below link for the End User Agreement:

www.umlib.nl/taverne-license

Take down policy

If you believe that this document breaches copyright please contact us at:

repository@maastrichtuniversity.nl

providing details and we will investigate your claim.
}

Copyright and moral rights for the publications made accessible in the public portal are retained by the authors and/or other copyright owners and it is a condition of accessing publications that users recognise and abide by the legal requirements associated with these

- Users may download and print one copy of any publication from the public portal for the purpose of private study or research.

- You may not further distribute the material or use it for any profit-making activity or commercial gain

If the publication is distributed under the terms of Article $25 \mathrm{fa}$ of the Dutch Copyright Act, indicated by the "Taverne" license above, 


\section{HOW CARDIAC MYOFIBERS KEEP PACE}

\section{Mathematical Modeling of Adaptive Myofiber \\ Reorientation and Electromechanics}

Marieke Lijnkamp-Pluijmert 
ISBN 978-90-9029213-7

Copyright (C) 2015 by M. Lijnkamp-Pluijmert

All rights reserved. No part of this publication may be produced, stored in a retrieval system, or transmitted, in any form, or by any means, electronically, mechanically, by print, photo print, recording or any other means without the prior written permission by the author.

Cover design by Vienna Romanée

Printed by Gildeprint, Enschede, The Netherlands 


\title{
HOW CARDIAC MYOFIBERS KEEP PACE
}

\author{
Mathematical Modeling of Adaptive Myofiber \\ Reorientation and Electromechanics
}

\begin{abstract}
PROEFSCHRIFT
ter verkrijging van de graad van doctor aan de Universiteit Maastricht, op gezag van de Rector Magnificus, Prof. dr. L.L.G. Soete volgens het besluit van het College van Decanen, in het openbaar te verdedigen op donderdag 24 september 2015 om 14:00u
\end{abstract}

door

\section{Marieke Hesther Pluijmert}

geboren op 3 september 1985,

te Woerden, Nederland 


\section{Promotores}

Prof. dr. T. Delhaas

Prof. dr. F.W. Prinzen

\section{Copromotor}

Dr. ir. P.H.M. Bovendeerd, Technische Universiteit Eindhoven

\section{Beoordelingscommissie}

Prof. dr. U. Schotten (voorzitter)

Prof. dr. H.-P. Brunner-La Rocca

Prof. dr. ir. R. Peeters

Prof. dr. N.H.J. Pijls (Technische Universiteit Eindhoven)

Prof. dr. ir. F.N. van de Vosse (Technische Universiteit Eindhoven)

Parts of the research described is this thesis were performed within the framework of CTMM, the Center for Translational Molecular Medicine, project COHFAR (COngestive Heart Failure and ARrhythmia; grant 01C-203).

Financial support by the Dutch Heart Foundation for the publication of this thesis is gratefully acknowledged.

Financial support for the publication of this thesis as provided by the Maastricht University, St. Jude Medical, and Biotronik is gratefully acknowledged. 



\section{Contents}

1 Background and aim of the thesis 9

1.1 General introduction . . . . . . . . . . . . . . . . . 10

1.2 Cardiac anatomy and physiology . . . . . . . . . . . 11

1.3 Cardiac disease . . . . . . . . . . . . . . . . . . 13

1.4 Towards model-assisted clinical decision-making for cardiac dis-

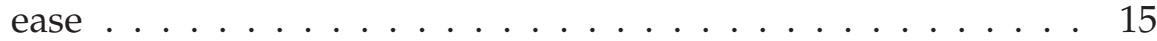

1.5 Aim and outline of the thesis . . . . . . . . . . . 17

2 Adaptive myofiber reorientation on the long term 25

2.1 Introduction . . . . . . . . . . . . . . . 27

2.2 Methods . . . . . . . . . . . . . . . . 27

2.3 Results ....................... . . 32

2.4 Discussion . . . . . . . . . . . . . . . . . 37

2.5 Conclusion . . . . . . . . . . . . . . . . . . . . 41

3 Myofiber reorientation in the situs inversus totalis left ventricle $\quad 45$

3.1 Introduction . . . . . . . . . . . . . . . 47

3.2 Methods . . . . . . . . . . . . . . . . . . . . 49

3.3 Results ...................... 56

3.4 Discussion . . . . . . . . . . . . . . . 57

3.5 Conclusion . . . . . . . . . . . . . . . . 66

4 Effect of activation and triaxial active stress development $\quad 71$

4.1 Introduction . . . . . . . . . . . . . . . 73

4.2 Methods ....................... . . 74

4.3 Results . . . . . . . . . . . . . . . 77

4.4 Discussion . . . . . . . . . . . . . . . . . . 84

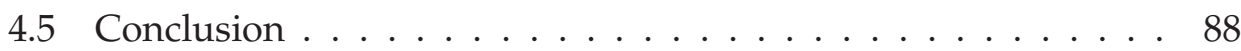

5 Determinants of biventricular cardiac function 91

5.1 Introduction . . . . . . . . . . . . . . . 93

5.2 Methods . . . . . . . . . . . . . . . . . . . 95

5.3 Results . . . . . . . . . . . . . . . . . . . . . 101 
5.4 Discussion . . . . . . . . . . . . . . . . . . 107

5.5 Conclusion . . . . . . . . . . . . . . . . . . . . 112

6 Left ventricular pacing site and response to CRT 117

6.1 Introduction . . . . . . . . . . . . . . . . . . . . 119

6.2 Methods . . . . . . . . . . . . . . . . . . . . . . . 119

6.3 Results . . . . . . . . . . . . . . . . . . . 121

6.4 Discussion . . . . . . . . . . . . . . . . . . . 127

6.5 Conclusion . . . . . . . . . . . . . . . . . . 131

7 General Discussion $\quad 135$

7.1 Introduction . . . . . . . . . . . . . . . 136

7.2 Adaptative reorientation as a tool to estimate myofiber orientation . . . . . . . . . . . . . . . . 136

7.3 Towards clinical application of computational models . . . . . 139

7.4 Future perspective . . . . . . . . . . . . . . . . . . 141

7.5 General Conclusions . . . . . . . . . . . . . . . . . 143

$\begin{array}{ll}\text { Summary } & 149\end{array}$

$\begin{array}{ll}\text { Samenvatting } & 153\end{array}$

$\begin{array}{ll}\text { Valorisatie } & 157\end{array}$

$\begin{array}{ll}\text { Dankwoord } & 161\end{array}$

$\begin{array}{ll}\text { About the author } & 165\end{array}$ 

Chapter 1

\section{Background and aim of the thesis}




\subsection{General introduction}

The heart is the pump of the cardiovascular system and it ensures blood to circulate throughout the body to exchange oxygen, nutrients, and metabolites. The pumping force is generated by contraction of the myocardial fibers. Contraction of the myofibers is initiated by electrical activation of myocardial cells. A specialized rapid conduction system ensures fast propagation of this electrical activity across the ventricular walls. Consequently, myofiber contraction is nearly synchronous, which is imperative for efficient pump function. On the other hand, abnormalities at any level in the complex system of the heart can prohibit efficient pump function.

In clinical practice, the diagnosis of cardiac pathology ideally consists of identifying the myocardial tissue abnormalities. However, direct assessment of tissue properties is difficult because clinical measurements give indirect information about the state of the heart. Hence, traditional diagnosis and treatment selection is based on interpretation of the clinical data in a statistical sense, using the experience of a clinician. In fact, it represents a best diagnosis and treatment for an average patient.

Mathematical models that describe the forward relation between cardiac tissue properties and clinical observations could serve as a useful clinical tool in determining pathology [30,58]. In an inverse analysis, model parameters would have to be adjusted iteratively to minimize differences between model predicted and clinically observed cardiac function. The spatial maps of tissue properties of that particular patient would then characterize the cardiac pathology. The clinician could take this information into account to arrive at a diagnosis and treatment selection that is more tailored to the patient at hand (figure 1.1). The inverse analysis, however, introduces a risk for nonuniqueness of the solution that may be decreased by increasing the number of clinical observations. Unfortunately, methods to assess relevant information are limited, both in number and accuracy. An alternative approach to overcome these limitations is to use knowledge on cardiac adaptation [4].

The aim of this thesis is to contribute to the procedure of model-assisted diagnosis. To this purpose, a mathematical model of biventricular (BiV) electromechanics was developed and applied for a clinically relevant case. We investigate whether input settings for myofiber orientation can be determined through adaptation. The model will be applied to gain additional insight in the mechanism behind Cardiac Resynchronization Therapy (CRT), a treatment for dyssynchronous heart failure patients, and how the benefit from this therapy can be maximized.

In this chapter, we briefly describe the cardiac anatomy and (patho) physiology, myofiber orientation and functional features thereof, and the clinical problems encountered with CRT. Subsequently, we elaborate on the develop- 


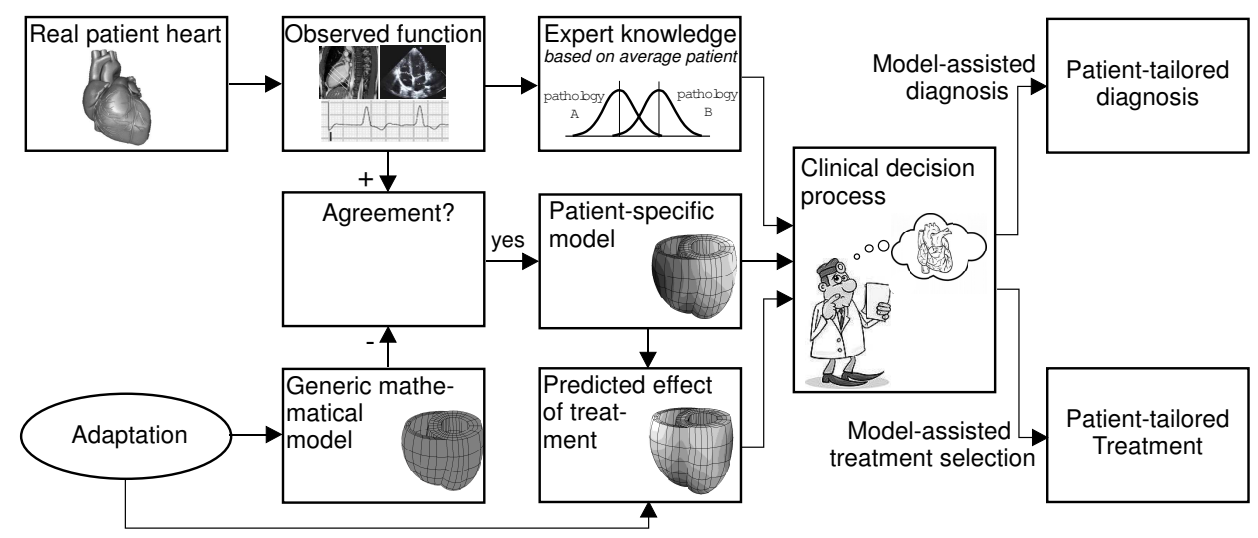

Figure 1.1: Flow chart for model-assisted diagnosis and treatment selection. In the diagnostic process, clinical measurements of the patients heart are evaluated in a statistical sense using the experience of the clinician. In model-assisted diagnosis, a patient-specific model is constructed by minimizing differences between model predicted and clinically observed cardiac function. The spatial maps of tissue properties from the patient-specific model can be used by the clinician as an extra source of information to arrive at a patient-tailored diagnosis and it can be used to evaluate the effect of candidate treatment options beforehand. Models of adaptation can be used both to estimate model parameters and to simulate the long-term effect of treatment.

ment and use of computational models to investigate cardiac electromechanical function and we conclude with the specific aims and outline of the thesis.

\subsection{Cardiac anatomy and physiology}

\subsubsection{Anatomy and pump function}

The heart contains four chambers: two atria and two ventricles (figure 1.2A). The atria collect blood that returns to the heart and facilitate filling of the ventricles. Electrical excitation of the ventricles results in simultaneous contraction of the left and right ventricle (LV and RV, respectively), and subsequently, ejection of blood into the arterial system.

Blood returning from the body enters the right atrium (RA). When pressure in the RV drops below RA pressure, the tricuspid valve opens and blood flows into the RV. Activation of the myofibers induces an immediate increase of RV cavity pressure. When pressure in the RV exceeds pressure in the pulmonary artery, the pulmonary valve opens and blood is pumped towards the lungs where it is oxygenized. Via the pulmonary veins, oxygenized blood is collected in the left atrium (LA). When pressure in the LV drops below the LA pressure, the mitral valve opens and blood flows into the LV. Again, acti- 
vation of the myofibers causes the LV cavity pressure to rise and the blood is pumped into the aorta towards the rest of the body.

\subsubsection{Geometry and Myofiber Orientation}

Geometrically, the LV approaches a thick-walled truncated ellipsoid. The relatively thin-walled RV is attached to the outer layers of the LV and covers about half of the LV outer surface. The ventricular cavities are separated by the interventricular septum. The atria and ventricles are separated by the basal plane.

Cardiac tissue consists of myocytes that are embedded in the extracellular matrix. Locally, the myocytes are aligned to form myofibers. In the ventricles, myofibers follow a specific orientation pattern that has an invariant nature among mammals, including humans [22, 23, 49, 61] (figure 1.2B). Near the endocardium, myofibers follow a right handed helical path. In the midwall, they are circumferentially oriented. And in the epicardium, they follow a left handed helical path. In addition, myofibers cross-over between the endo- and epicardium.

Alignment of myofibers in cardiac muscle tissue is substantially different from alignment of myofibers in skeletal muscle tissue. The structural features of cardiac myofiber orientation play an important role in the mechanical function of the ventricular wall. Due to the large tissue anisotropy as induced during myofiber contraction, the myofiber orientation strongly affects wall deformation. An important deformation mode typical for the left ventricle is torsion, i.e. the rotation of the apex with respect to the base. Under the assumption that cells are operating in the same range for values of stress and strain, little differences in myofiber shortening across the wall during ejection are expected. Torsion compensates for the difference in shortening between endocardial and epicardial myofibers that would occur from decrease in cavity volume alone [5].

\subsubsection{Electrical wave propagation}

A normal heartbeat is initiated by electrical stimulation of the myocytes. The electrical signal originates from the sinoatrial (SA) node, located at the right atrium. The SA node consists of natural pacemaker cells, which generate action potentials (APs) about 70 times per minute. Once an AP is generated, activation spreads throughout the atria and is captured by the atrioventricular $(\mathrm{AV})$ node. The AV node is the only conducting path between the atria and ventricles. Conduction of the impulse through the AV node occurs relatively slow. The later activation of the ventricles with respect to the atria ensures ejection of blood into the ventricles first before the ventricles start to contract. 
A Global anatomy

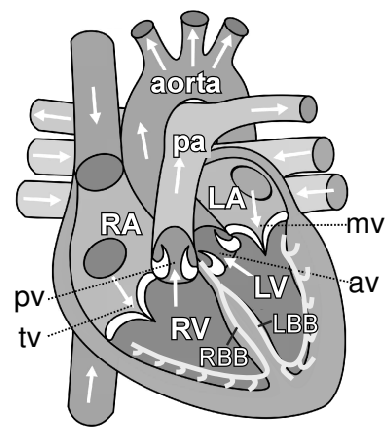

B Myofiber orientation

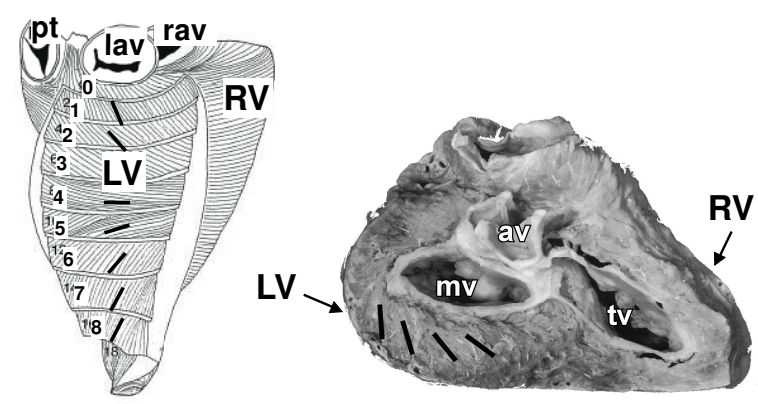

Figure 1.2: Basic anatomy and physiology of the heart. A: Cross-section of the heart along a long axis showing the four cavities. The white arrows indicate the direction of blood flow. LA=left atrium; LV=left ventricle; $R A=$ right atrium; RV=right ventricle; av=aortic valve; $\mathrm{mv}=$ mitral valve; $\mathrm{pv}=$ pulmonary valve; $\mathrm{tv}=$ tricuspid valve; pa=pulmonary artery; $\mathrm{LBB}=$ left bundle branch; $\mathrm{RBB}=$ right bundle branch. B: Direction of the myofibers in the LV at several heights between base and apex, and several depths from epicardium (0) to endocardium (8) [44] (left). The myofiber orientation changes from a left handed helical path near the epicardium (0-2), to circumferentially oriented near the midwall (3-5), and a right handed helical path near the endocardium (6-8). Short-axis cross-section near the base showing cross-over of myofiber between endo- and epicardium [3] (right).

Distal to the AV node, activation enters the ventricles in the specialized rapid conduction system. First, it travels through the bundle of His that separates in two main bundles, the left and right bundle branch (LBB and RBB, respectively). Further distally, the LBB and RBB ramify in Purkinje fibers. The closeending of the Purkinje-fibers near the endocardium ensures a fast and rather simultaneous activation of the (sub)endocardium. Subsequently the activation travels much slower from cell-to-cell in the working myocardium to the epicardium. It takes about $70 \mathrm{~ms}$ to activate the ventricles of a healthy human heart. An example of sequence of activation in the ventricles of a normal human heart is illustrated in the left panel of figure 1.3 [17]. The electrocardiogram (ECG) is a noninvasive recording of the electrical activity of the heart. Activation of the ventricles is reflected in the QRS-complex.

\subsection{Cardiac disease}

\subsubsection{Dyssynchronous Heart Failure}

Cardiac disease leading to inefficient pump function has various causes and forms. One form is dyssynchronous heart failure (DHF), associated with ab- 


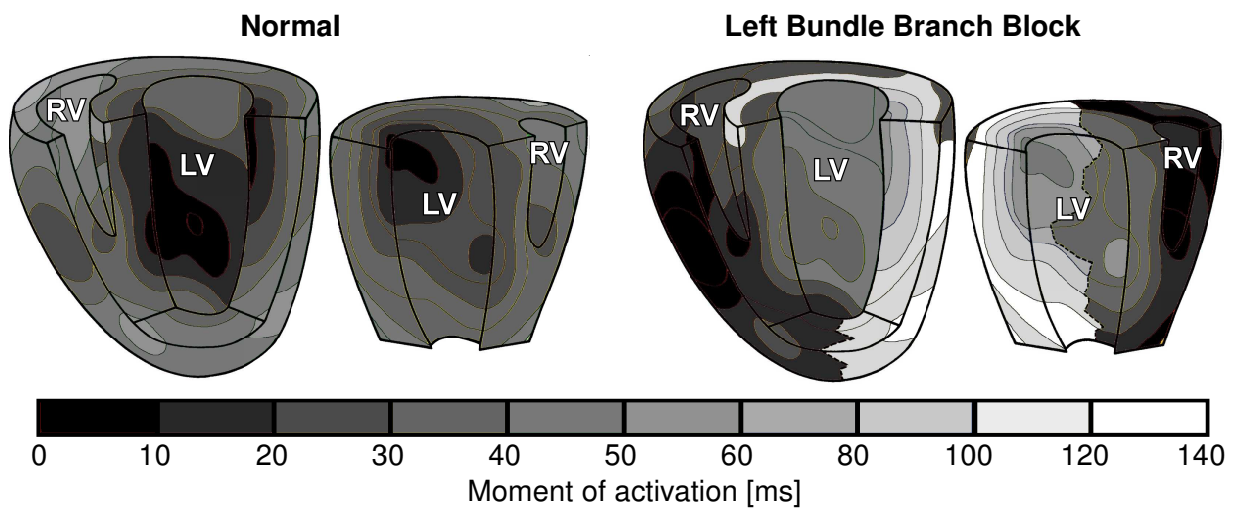

Figure 1.3: Sequence of electrical activation in the ventricular walls during normal activation (left) and left bundle branch block (right) [17].

normally slow ventricular impulse conduction. Disabled conduction through the LBB, i.e., left bundle branch block (LBBB), is a common conduction disorder in DHF. In LBBB, onset of ventricular activation starts in the RV and activation propagates slowly from cell-to-cell to the LV causing delayed activation of the LV free wall (LVfw) (figure 1.3, right panel). Contraction of the RV and septum precedes contraction of the LVfw. These regional differences in mechanical function are associated with discoordinated contraction, impairment of cardiac pump function, adverse structural remodeling, and increased risk of heart failure $[60,62,73]$.

\subsubsection{Cardiac Resynchronization Therapy}

At the end of the 1990s, it became apparent that ventricular pacing can improve cardiac function in DHF patients $[8,9,31]$. Biventricular (BiV) pacing should resynchronize electrical activation of the RV and LV. Subsequently, large clinical trials have shown that $\mathrm{BiV}$ pacing or cardiac resynchronization therapy (CRT) benefits the majority of moderate-to-severe HF patients with reduced ejection fraction and prolonged QRS duration [1, 12]. Yet, up to half of the patients included in CRT trails fail to show significant improvement of symptoms related to DHF $[1,13]$. This high number of non-responders emphasizes the need for improved strategies to achieve maximal benefit of CRT. Such strategies consist of optimizing 1) patient selection, 2) the position of the pacing leads, and 3) the settings of the pacing device.

Important determinants of the clinical benefit of CRT are the electrical and structural substrate and the site of implantation. While clinical studies and experimental work have provided a large amount of evidence for certain approaches $[38,55,59,63]$, evidence is lacking in some areas. For example, it re- 
mains unclear whether to position the LV lead in a specific anatomical region $[18,19,45,55,59]$ or in a region characterized by specific electrical or mechanical properties [21, 38, 74, 75]. Therefore, improved understanding is needed of the relation between pump function and ventricular activation sequence as induced by the location of the LV electrode. However, detailed electrical mapping in patients is difficult because of its highly invasive nature. In addition, quantitative information on local tissue variables, e.g. tissue stress and contractility, cannot be obtained reliably in vivo [29]. It is here that mathematical models based on physical and physiological principles can help to gain additional insight in the relation between pacing mode and pump function.

\subsection{Towards model-assisted clinical decision-making for cardiac disease}

Most of current knowledge on cardiac (patho)physiology is derived from in vivo and in vitro experiments. Additionally, in silico research on cardiac physiology is growing. The use of biophysical models containing local cardiac tissue composition and structure as well as global organ shape can give additional insight into the forward relation between cardiac tissue properties and pump function. Nowadays, models of cardiac electromechanics are evolving to a state where they can assist in clinical decision making and treatment selection, e.g., by predicting response to CRT $[15,47,56]$.

\subsubsection{Models of electrophysiology}

The entire process of electromechanical activation starts with the action potential (AP). Current mathematical models to describe the AP are based on the approach proposed by Hodgkin and Huxley [27]. Once the AP is generated, it propagates over neighboring cells. The propagation is often described using a bidomain model that describes the extracellular and transmembrane potential as function of the location in the wall and time. Bidomain models can simulate the effects of external stimuli and defibrillation currents [65]. When the bidomain model is applied to cardiac tissue surrounded by a model of the thorax, it can be used to compute the surface ECG [51].

Due to the steepness of the action potential upstroke and the resulting steep spatial potential gradients, bidomain models need high temporal and spatial resolution. Consequently, published electrophysiological models have 10 to 100 million elements $[25,52]$ and, hence, they are computationally expensive. Therefore, coupling of these models of electrophysiology to models of cardiac mechanics, i.e., relating electrical activity to active stress development in the sarcomeres, is not yet feasible for clinical application. As an alter- 
native approach to model electromechanical coupling, the bidomain model is often simplified to the monodomain model. This monodomain model is then weakly coupled to a model of cardiac mechanics, i.e., electrical activation sequence is computed separately from cardiac mechanics $[25,26,47,70,69]$. The first computational studies have been published that offer some new clinically relevant insights in the relation between biventricular pacing and response to CRT $[15,36,48]$.

If details with respect to the ion-currents and membrane potential are not taken into account and impulse propagation alone is to be computed, the Eikonal model can be used. This equation was derived from the bidomain model by Colli-Franzone et al. [14] and solves for the spatial variation of electrical activation times. The timing of activation is used to initiate active stress development in a model of cardiac mechanics. This approach is computationally less demanding and has been successfully applied in models of cardiac mechanics to simulate cardiac electromechanics $[32,33,34,37,56]$.

\subsubsection{Models of mechanics}

The deformation of the heart during the cardiac cycle is determined by the mechanical equilibrium between forces related to pressure in the cardiac cavities, passive stretch of the connective tissue matrix, and active contraction of the myofibers. The finite element (FE) method enables evaluation of this equilibrium in three dimensions. The first FE models of cardiac mechanics contained the LV only [11, 24, 46, 68]. The LV has a well-defined shape that is easy to mesh, and resulting computation times were acceptable for this reduced geometry. Nowadays, geometrically more complex models of BiV cardiac mechanics have been developed [2, 15, 35, 47, 54, 67].

While FE computations can be performed with high accuracy, the predictive value of the computational results is limited by the accuracy at which the model input parameters can be experimentally measured (figure 1.1). Geometrical characteristics can be captured relatively easy from imaging data and included into models through sophisticated meshing algorithms [41]. Experimental measurement of other input parameters, such as myofiber orientation or material properties, is more challenging. While first steps have been made to measure myofiber orientations in vivo using magnetic resonance diffusion tensor imaging (MRDTI) [64], researchers still have to rely on generic data from in vitro MRDTI [57] or histological measurements [42, 49]. Thereby, the inaccuracy introduced into the model is twofold. First, it has been questioned whether these techniques measure myofiber orientation with sufficient accuracy [20]. And second, the generic data is morphed into a patient-specific geometry. Considering the high sensitivity of the spatial variation in myofiber stress and strain for the choice of myofiber orientation within the reported 
range of experimental data $[11,10,66]$, these inaccuracies might strongly determine the model output.

Another approach for determining input parameters would be to consider the heart as an adaptive organ [4]. Subsequently, adaptation rules would then describe a local feedback mechanism from mechanical load to changes in tissue properties. A clear example of cardiac adaptation is the global change in ventricular wall mass and cavity volume in response to changes in hemodynamic loading $[16,28,50]$. In addition, it has been suggested that myofiber orientation is controlled to ensure homogeneous distribution of mechanical load throughout the wall [7, 43, 71]. The hypothesis of homogeneous distribution of myofiber stress and strain was used in a mathematical model of adaptation, and indeed, realistic values for LV geometry and myofiber orientation were estimated [6].

This hypothesis was first tested by optimizing myofiber orientation for minimal heterogeneity in myofiber shortening during ejection, sarcomere length, and myofiber stress [53, 72]. Orientations thus obtained compared favorably with those measured experimentally. As it is more likely that cells adapt in response to local mechanical stimuli [6], Kroon et al. [39] developed a model in which myofiber orientation changed in response to local fiber crossfiber shear strain. Their model was also capable of predicting a realistic LV structure together with a significant increase of homogenization of mechanical load throughout the LV wall. In addition, pump work was increased in the adapted LV structure, while differences between measured and model computed deformation were significantly reduced [40].

\subsection{Aim and outline of the thesis}

The aim of this thesis is to contribute to the procedure of model-assisted clinical decision-making for cardiac disease. To this purpose a biventricular (BiV) finite element model of cardiac electromechanics is developed and applied. In light of the difficulty to measure myofiber orientation with sufficient accuracy, we investigated whether the adaptation model developed by Kroon et al. [39] could be used to obtain a more accurate estimate for the myofiber orientation.

In chapter 2, we build upon the hypothesis of shear-induced myofiber reorientation, proposed by [39] et al. and further investigate the computational model with respect to the effect of initial and boundary conditions at the base on long-term outcome of the adaptive process.

Besides the normal heart, we also consider the LV in a mirror-imaged position of organs, i.e. Situs Inversus Totalis (SIT). The SIT LV has quite an abnormal myofiber orientation pattern with respect to the normal heart. As the SIT heart consists of the same type of cells as the normal heart, a similar stimulus- 
response relation is expected. In chapter 3, we used the SIT LV as an extreme test case to explore whether myofiber orientations of the SIT LV can also be predicted by shear-induced myofiber reorientation.

In chapter 4 we investigate whether remaining differences between computed and measured patterns of circumferential-radial shear strain are caused by limitations in the LV mechanics model. Therefore, a physiological sequence of activation and active stress development in not only fiber but also cross-fiber direction were included into the model. These two phenomenons are known to directly affect the myocardial shear deformation, and thus, indirectly affect the process of shear-induced myofiber reorientation.

For use of FE models to assist in clinical decision-making, models including both RV and LV are needed. In chapter 5 , a BiV model of cardiac mechanics is presented. We investigate the sensitivity of cardiac function to geometry and myofiber orientation. A parameterized description of the BiV geometry enabled well-defined variations of the geometry. In addition, myofiber orientations are changed through shear-induced myofiber reorientation.

In chapter 6 , the $\mathrm{BiV}$ mechanics model with adapted myofiber orientation is combined with the Eikonal model for electrical wave propagation [32]. The combined model enables simulation of cardiac function during normal activation, in case of $\mathrm{LBBB}$ and during $\mathrm{BiV}$ pacing. We study the relation between $\mathrm{LV}$ pacing site during biventricular $(\mathrm{BiV})$ pacing and cardiac function.

This thesis concludes with a general discussion in chapter 7 in which the findings of the various chapters are put into a broader context. 


\section{References}

1. Abraham WT (2002). "Cardiac resynchronization therapy for heart failure: biventricular pacing and beyond." Curr Opin Cardiol 17.4, pp. 346-352.

2. Aguado-Sierra J, Krishnamurthy A, Villongco C, Chuang J, Howard E, Gonzales MJ, Omens J, Krummen DE, Narayan S, Kerckhoffs RCP, and McCulloch AD (2011). "Patientspecific modeling of dyssynchronous heart failure: a case study." Prog Biophys Mol Biol 107.1, pp. 147-155.

3. Anderson R and Becker A (1980). "Cardiac Anatomy". Gowes Medical Publishing.

4. Arts T, Bovendeerd P, Delhaas T, and Prinzen F (2003). "Modeling the relation between cardiac pump function and myofiber mechanics". J Biomech 36, pp. 731-736.

5. Arts T, Meerbaum S, Reneman RS, and Corday E (1984). "Torsion of the left ventricle during the ejection phase in the intact dog." Cardiovasc Res 18.3, pp. 183-193.

6. Arts T, Prinzen FW, Snoeckx LH, Rijcken JM, and Reneman RS (1994). "Adaptation of cardiac structure by mechanical feedback in the environment of the cell: a model study". Biophysical Journal 66, pp. 953-961.

7. Arts T, Veenstra PC, and Reneman RS (1982). "Epicardial deformation and left ventricular wall mechanisms during ejection in the dog." Am J Physiol 243.3, H379-H390.

8. Auricchio A, Stellbrink C, Block M, Sack S, Vogt J, Bakker P, Klein H, Kramer A, Ding J, Salo R, Tockman B, Pochet T, and Spinelli J (1999). "Effect of pacing chamber and atrioventricular delay on acute systolic function of paced patients with congestive heart failure. The Pacing Therapies for Congestive Heart Failure Study Group. The Guidant Congestive Heart Failure Research Group." Circulation 99.23, pp. 2993-3001.

9. Blanc JJ, Etienne Y, Gilard M, Mansourati J, Munier S, Boschat J, Benditt DG, and Lurie KG (1997). "Evaluation of different ventricular pacing sites in patients with severe heart failure: results of an acute hemodynamic study." Circulation 96.10, pp. 3273-3277.

10. Bovendeerd PHM, Kroon W, and Delhaas T (2009). "Determinants of left ventricular shear strain". Am J Physiol - Heart Circ Physiol 297.3, H1058-H1068.

11. Bovendeerd P, Arts T, Huyghe J, Campen D van, and Reneman R (1992). "Dependency of local left ventricular wall mechanics on myocardial myofiber orientation: a model study". $J$ Biomech 25, pp. 1129-1135.

12. Cazeau S, Leclercq C, Lavergne T, Walker S, Varma C, Linde C, Garrigue S, Kappenberger L, Haywood GA, Santini M, Bailleul C, Daubert JC, and Multisite Stimulation in Cardiomyopathies (M. U. S. T. I. C) Study Investigators (2001). "Effects of multisite biventricular pacing in patients with heart failure and intraventricular conduction delay." N Engl J Med 344.12, pp. 873-880.

13. Cleland JGF, Daubert JC, Erdmann E, Freemantle N, Gras D, Kappenberger L, Tavazzi L, and C A R E-H F Study Investigators (2005). "The effect of cardiac resynchronization on morbidity and mortality in heart failure." N Engl J Med 352.15, pp. 1539-1549.

14. Colli Franzone P, Guerri L, and Tentoni S (1990). "Mathematical Modeling of the Excitation Process in Myocardial Tissue: Influence of Fiber Rotation on Wavefront Propagation and 
Potential Field". Mathematical Biosciences 101, pp. 155-235.

15. Constantino J, Hu Y, and Trayanova NA (2012). "A computational approach to understanding the cardiac electromechanical activation sequence in the normal and failing heart, with translation to the clinical practice of CRT.". Prog Biophys Mol Biol 110.2-3, pp. 372-379.

16. Donker DW, Volders PGA, Arts T, Bekkers BCAM, Hofstra L, Spätjens RLHMG, Beekman JDM, Borgers M, Crijns HJGM, and Vos MA (2005). “End-diastolic myofiber stress and ejection strain increase with ventricular volume overload-Serial in-vivo analyses in dogs with complete atrioventricular block." Basic Res Cardiol 100.4, pp. 372-382.

17. Durrer D, van Dam RT, Freud GE, Janse MJ, Meijler FL, and Arzbaecher RC (1970). “Total excitation of the isolated human heart." Circulation 41.6, pp. 899-912.

18. Foley PWX, Chalil S, Ratib K, Smith R, Prinzen F, Auricchio A, and Leyva F (2011). "Fluoroscopic left ventricular lead position and the long-term clinical outcome of cardiac resynchronization therapy." Pacing Clin Electrophysiol 34.7, pp. 785-797.

19. Fung JWH, Lam YY, Zhang Q, Yip GWK, Chan WWL, Chan GCP, Chan JYS, and Yu CM (2009). "Effect of left ventricular lead concordance to the delayed contraction segment on echocardiographic and clinical outcomes after cardiac resynchronization therapy." J Cardiovasc Electrophysiol 20.5, pp. 530-535.

20. Geerts-Ossevoort L, Kerckhoffs R, Bovendeerd P, and Arts T (2003). “Towards Patient Specific Models of Cardiac Mechanics: A Sensitivity Study". In: Functional Imaging and Modeling of the Heart. Ed. by I Magnin, J Montagnat, P Clarysse, J Nenonen, and T Katila. Vol. 2674. Lecture Notes in Computer Science. Springer Berlin Heidelberg, pp. 81-90.

21. Gold MR, Leman RB, Wold N, Sturdivant JL, and Yu Y (2014). “The effect of left ventricular electrical delay on the acute hemodynamic response with cardiac resynchronization therapy." J Cardiovasc Electrophysiol 25.6, pp. 624-630.

22. Greenbaum R, Ho S, Gibson D, Becker A, and Anderson R (1981). "Left ventricular fibre architecture in man". Br Heart J 45, pp. 248-263.

23. Grimm A, Katele K, and Lin H (1976). "Fiber bundle direction in the mammalian heart. An extension of the 'nested shells' model." Basic Res. Cardiol. 71, pp. 381-388.

24. Guccione J, Costa K, and McCulloch A (1995). "Finite element stress analysis of left ventricular mechanics in the beating dog heart." J Biomech 28.10, pp. 1167-1177.

25. Gurev V, Constantino J, Rice JJ, and Trayanova NA (2010). "Distribution of electromechanical delay in the heart: insights from a three-dimensional electromechanical model." Biophys J 99.3, pp. 745-754.

26. Gurev V, Lee T, Constantino J, Arevalo H, and Trayanova NA (2011). "Models of cardiac electromechanics based on individual hearts imaging data: image-based electromechanical models of the heart." Biomech Model Mechanobiol 10.3, pp. 295-306.

27. Hodgkin AL and Huxley AF (1952). "A quantitative description of membrane current and its application to conduction and excitation in nerve." J Physiol 117.4, pp. 500-544.

28. Holmes JW (2004). "Candidate mechanical stimuli for hypertrophy during volume overload." J Appl Physiol (1985) 97.4, pp. 1453-1460.

29. Huisman RM, Elzinga G, Westerhof N, and Sipkema P (1980). "Measurement of left ventricular wall stress." Cardiovasc Res 14.3, pp. 142-153.

30. Hunter PJ, Coveney PV, De Bono B, Diaz V, Fenner J, Frangi AF, Harris P, Hose R, Kohl $\mathrm{P}$, Lawford P, et al. (2010). "A vision and strategy for the virtual physiological human in 2010 and beyond". Phil Trans R Soc Lond A 368, pp. 2595-2614.

31. Kass DA, Chen CH, Curry C, Talbot M, Berger R, Fetics B, and Nevo E (1999). “Improved left ventricular mechanics from acute VDD pacing in patients with dilated cardiomyopathy and ventricular conduction delay." Circulation 99.12, pp. 1567-1573.

32. Kerckhoffs RCP, Bovendeerd PHM, Kotte JCS, Prinzen FW, Smits K, and Arts T (2003a).

"Homogeneity of cardiac contraction despite physiological asynchrony of depolarization: a model study." Ann Biomed Eng 31.5, pp. 536-547. 
33. Kerckhoffs RCP, Bovendeerd PHM, Prinzen FW, Smits K, and Arts T (2003b). “Intraand interventricular asynchrony of electromechanics in the ventricularly paced heart". J Eng Math 47, pp. 201-216.

34. Kerckhoffs RCP, Faris OP, Bovendeerd PHM, Prinzen FW, Smits K McVeigh ER, and Arts $\mathrm{T}$ (2005). "Electromechanics of paced left ventricle simulated by straightforward mathematical model: comparison with experiments". Am J Physiol - Heart Circ Physiol 289, H1889-H1897.

35. Kerckhoffs RCP, Healy SN, Usyk TP, and D MA (2006). "Computational Methods for Cardiac Electromechanics". J PROC IEEE 94.4, pp. 769-783.

36. Kerckhoffs RCP, McCulloch AD, Omens JH, and Mulligan LJ (2009). “Effects of biventricular pacing and scar size in a computational model of the failing heart with left bundle branch block". Med Im Anal 13, pp. 362-369.

37. Kerckhoffs RCP, Faris OP, Bovendeerd PHM, Prinzen FW, Smits K, McVeigh ER, and Arts T (2003c). "Timing of depolarization and contraction in the paced canine left ventricle: model and experiment." J Cardiovasc Electrophysiol 14.10 Suppl, S188-S195.

38. Khan FZ, Virdee MS, Palmer CR, Pugh PJ, O'Halloran D, Elsik M, Read PA, Begley D, Fynn SP, and Dutka DP (2012). “Targeted left ventricular lead placement to guide cardiac resynchronization therapy: the TARGET study: a randomized, controlled trial." J Am Coll Cardiol 59.17, pp. 1509-1518.

39. Kroon W, Delhaas T, Arts T, and Bovendeerd P (2009a). “Computational analysis of the myocardial structure: Adaptation of myofiber orientations through deformation in three dimensions". Med Imag Anal 13, pp. 346-353.

40. Kroon W, Delhaas T, Bovendeerd P, and Arts T (2009b). "Adaptive Reorientation of Cardiac Myofibers: Comparison of Left Ventricular Shear in Model and Experiment". In: Functional Imaging and Modeling of the Heart. Ed. by N Ayache, H Delingette, and M Sermesant. Vol. 5528. Lecture Notes in Computer Science. Springer Berlin Heidelberg, pp. 58-67.

41. Lamata P, Sinclair M, Kerfoot E, Lee A, Crozier A, Blazevic B, Land S, Lewandowski AJ, Barber D, Niederer S, and Smith N (2014). "An automatic service for the personalization of ventricular cardiac meshes." J R Soc Interface 11.91, p. 20131023.

42. Legrice IJ, Hunter PJ, and Smaill BH (1997). "Laminar structure of the heart: a mathematical model." Am J Physiol 272.5, H2466-H2476.

43. MacGowan GA, Shapiro EP, Azhari H, Siu CO, Hees PS, Hutchins GM, Weiss JL, and Rademakers FE (1997). "Noninvasive measurement of shortening in the fiber and cross-fiber directions in the normal human left ventricle and in idiopathic dilated cardiomyopathy". Circulation 96 , pp. 535-541.

44. Matsumura H, Aizawa Y, and Kumaki K (1990). "Myocardial architecture in situs inversus vicerum totalis". Clark EB, Takao A (Eds) Developmental cardiology: morphogenesis and function, Futura Pub Co, Mount Kisco ISBN-13: 978-0-879-93382-1. Ed. by E Clark and A Takao, pp. 605-624.

45. Merchant FM, Heist EK, McCarty D, Kumar P, Das S, Blendea D, Ellinor PT, Mela T, Picard MH, Ruskin JN, and Singh JP (2010). "Impact of segmental left ventricle lead position on cardiac resynchronization therapy outcomes." Heart Rhythm 7.5, pp. 639-644.

46. Nash MP and Hunter PJ (2000). "Computational mechanics of the heart". J Elasticity 61, pp. 113-141.

47. Niederer SA, Plank G, Chinchapatnam P, Ginks M, Lamata P, Rhode KS, Rinaldi CA, Razavi R, and Smith NP (2011). "Length-dependent tension in the failing heart and the efficacy of cardiac resynchronization therapy". Cardiovascular Research 89(2), pp. 336-343.

48. Niederer SA, Lamata P, Plank G, Chinchapatnam P, Ginks M, Rhode K, Rinaldi CA, Razavi R, and Smith NP (2012). "Analyses of the redistribution of work following cardiac resynchronisation therapy in a patient specific model." PLoS One 7.8, e43504.

49. Nielsen PM, LeGrice IJ, Smaill BH, and Hunter PJ (1991). "Mathematical model of geometry and fibrous structure of the heart". Am J Physiol - Heart Circ Physiol 260, H1365-H1378. 
50. Omens JH (1998). "Stress and strain as regulators of myocardial growth." Prog Biophys Mol Biol 69.2-3, pp. 559-572.

51. Potse M, Dubé B, Richer J, Vinet A, and Gulrajani RM (2006). “A comparison of monodomain and bidomain reaction-diffusion models for action potential propagation in the human heart." IEEE Trans Biomed Eng 53.12 Pt 1, pp. 2425-2435.

52. Potse M, Krause D, Bacharova L, Krause R, Prinzen FW, and Auricchio A (2012). "Similarities and differences between electrocardiogram signs of left bundle-branch block and leftventricular uncoupling." Europace 14 Suppl 5, pp. v33-v39.

53. Rijcken JM, Bovendeerd PHM, Schoofs AJG, Van Campen DH, and Arts T (1999). “Optimization of cardiac fiber orientation for homogeneous fiber strain during ejection". Ann Biomed Eng 27, pp. 289-297.

54. Saint-Marie J, Chapelle D, Cimrman R, and Sorine M (2006). “Modeling and estimation of the cardiac electromechanical activity". Comp and Struc 84, pp. 1743-1759.

55. Saxon LA, Olshansky B, Volosin K, Steinberg JS, Lee BK, Tomassoni G, Guarnieri T, Rao A, Yong P, Galle E, Leigh J, Ecklund F, and Bristow MR (2009). "Influence of left ventricular lead location on outcomes in the COMPANION study." J Cardiovasc Electrophysiol 20.7, pp. 764 768 .

56. Sermesant M, Chabiniok R, Chinchapatnam P, Mansi T, Billet F, Moireau P, Peyrat JM, Wong K, Relan J, Rhode K, et al. (2012). "Patient-specific electromechanical models of the heart for the prediction of pacing acute effects in CRT: a preliminary clinical validation." Med Image Anal 16.1, pp. 201-215.

57. Sermesant M, Billet F, Chabiniok R, Mansi T, Chinchapatnam P, Moireau P, Peyrat JM, Rhode K, Ginks M, Lambiase P, et al. (2009). "Personalised Electromechanical Model of the Heart for the Prediction of the Acute Effects of Cardiac Resynchronisation Therapy". In: Functional Imaging and Modeling of the Heart. Ed. by N Ayache, H Delingette, and M Sermesant. Vol. 5528. Lecture Notes in Computer Science. Springer Berlin Heidelberg, pp. 239-248.

58. Sermesant M, Peyrat JM, Chinchapatnam P, Billet F, Mansi T, Rhode K, Delingette H, Razavi R, and Ayache N (2008). "Toward patient-specific myocardial models of the heart." Heart Fail Clin 4.3, pp. 289-301.

59. Singh JP, Klein HU, Huang DT, Reek S, Kuniss M, Quesada A, Barsheshet A, Cannom D, Goldenberg I, McNitt S, Daubert JP, Zareba W, and Moss AJ (2011). "Left ventricular lead position and clinical outcome in the multicenter automatic defibrillator implantation trial-cardiac resynchronization therapy (MADIT-CRT) trial." Circulation 123.11, pp. 1159-1166.

60. Spragg DD, Akar FG, Helm RH, Tunin RS, Tomaselli GF, and Kass DA (2005). “Abnormal conduction and repolarization in late-activated myocardium of dyssynchronously contracting hearts." Cardiovasc Res 67.1, pp. 77-86.

61. Streeter Jr D, Spotnitz HM, Patel DP, Ross Jr J, and Sonnenblick EH (1969). "Fiber orientation in the canine left ventricle during diastole and systole." Circ Res 24.3, pp. 339-347.

62. Sweeney MO, Hellkamp AS, Ellenbogen KA, Greenspon AJ, Freedman RA, Lee KL, Lamas GA, and for the MOde Selection Trial (MOST) Investigators (2003). "Adverse effect of ventricular pacing on heart failure and atrial fibrillation among patients with normal baseline QRS duration in a clinical trial of pacemaker therapy for sinus node dysfunction." Circulation 107.23, pp. 2932-2937.

63. Thébault C, Donal E, Meunier C, Gervais R, Gerritse B, Gold MR, Abraham WT, Linde C, Daubert JC, and R.E.V.E.R.S.E. Study Group (2012). "Sites of left and right ventricular lead implantation and response to cardiac resynchronization therapy observations from the REVERSE trial." Eur Heart J 33.21, pp. 2662-2671.

64. Toussaint N, Sermesant M, Stoeck CT, Kozerke S, and Batchelor PG (2010). "In vivo human 3D cardiac fibre architecture: reconstruction using curvilinear interpolation of diffusion tensor images." Med Image Comput Comput Assist Interv 13.Pt 1, pp. 418-425.

65. Trayanova NA, Constantino J, and Gurev V (2010). "Models of stretch-activated ventric- 
ular arrhythmias." J Electrocardiol 43.6, pp. 479-485.

66. Ubbink SWJ, Bovendeerd PHM, Delhaas T, Arts T, and Van de Vosse FN (2006). "Towards model-based analysis of cardiac MR tagging data: Relation between left ventricular shear strain and myofiber orientation". Med Imag Anal 10, pp. 623-641.

67. Usyk TP, LeGrice IJ, and McCulloch AD (2002). "Computational model three dimensional cardiac electromechanics". Comp Visual Sci 4, pp. 249-257.

68. Usyk TP, Mazhari R, and McCulloch AD (2000). "Effect of laminar orthotropic myofiber architecture on regional stress and strain in the canine left ventricle". J Elasticity 61, pp. 143164.

69. Usyk TP and McCulloch AD (2003a). "Electromechanical model of cardiac resynchronization in the dilated failing heart with left bundle branch block". J Electrocardiol 36, pp. 5761.

70. Usyk TP and McCulloch A (2003b). “Relationship Between Regional Shortening and Asynchronous Electrical Activation in a Three-Dimensional Model of Ventricular Electromechanics". Journal of Cardiovascular Electrophysiology 14, S196-S202.

71. van der Vusse GJ, Arts T, Glatz JF, and Reneman RS (1990). "Transmural differences in energy metabolism of the left ventricular myocardium: fact or fiction." J Mol Cell Cardiol 22.1, pp. 23-37.

72. Vendelin M, Bovendeerd PHM, Engelbrecht J, and Arts T (2002). “Optimizing ventricular fibers: uniform strain of stress, but not ATP consumption, leads to high efficiency". Am J Physiol - Heart Circ Physiol 283, H1072-H1081.

73. Vernooy K, Verbeek XAAM, Peschar M, Crijns HJGM, Arts T, Cornelussen RNM, and Prinzen FW (2005). "Left bundle branch block induces ventricular remodelling and functional septal hypoperfusion." Eur Heart J 26.1, pp. 91-98.

74. Ypenburg C, van Bommel RJ, Delgado V, Mollema SA, Bleeker GB, Boersma E, Schalij MJ, and Bax JJ (2008). “Optimal left ventricular lead position predicts reverse remodeling and survival after cardiac resynchronization therapy." J Am Coll Cardiol 52.17, pp. 1402-1409.

75. Zanon F, Baracca E, Pastore G, Fraccaro C, Roncon L, Aggio S, Noventa F, Mazza A, and Prinzen F (2014). "Determination of the longest intrapatient left ventricular electrical delay may predict acute hemodynamic improvement in patients after cardiac resynchronization therapy." Circ Arrhythm Electrophysiol 7.3, pp. 377-383. 
Chapter 1 


\section{Chapter 2}

\section{Adaptive myofiber reorientation on the long term}

The contents of this chapter are based on:

M. Pluijmert, W. Kroon, T. Delhaas, P.H.M. Bovendeerd, Adaptive reorientation of cardiac myofibers: the long-term effect of initial and boundary conditions, Mechanics Research Communications, 42:60-67, 2012 


\section{Abstract}

On the basis of results from modeling and experimental studies it has been hypothesized that myocytes adapt their orientation to achieve a preferred mechanical load. In a previous computational model study in which fiber reorientation was considered as a local response to local fiber cross-fiber shear strain, we have shown that predicted left ventricular (LV) myofiber orientations agreed well with experimental data. In this study, in the latter model we investigated the effect of initial and boundary conditions on predicted fiber orientations on the long term. After adaptation, predicted fiber orientation and deformation became more realistic, irrespective of initial and boundary conditions. As adaptation proceeded, the effect of initial conditions was found to disappear, suggesting that one single optimal fiber orientation field exists for the heart. In contrast, the effect of the boundary conditions persisted, indicating that modeling of in particular the interaction between myocardium and valvular annulus is relevant for predicting LV myofiber reorientation. 


\subsection{Introduction}

Several finite element (FE) models have been developed that are capable of describing the forward relation between cardiac tissue properties and clinically assessable parameters of pump (dys)function [2, 3, 8]. Gradually, attempts are made to enhance clinically obtained diagnostic information by employing these computational models in an inverse mode [7]. In addition, the models might be used to assist in treatment selection, by simulating the acute effect of candidate interventions beforehand. As interventions may cause cardiac adaptation on the long term, the current models should be extended with a description of the adaptive properties of the myocardium.

Previous model studies have shown that strain distribution in the cardiac wall is highly dependent on myofiber orientation $[2,16]$. In experimental studies a change in myofiber orientation was observed when mechanical properties of the surrounding tissue were altered $[19,21]$. In addition, myofiber strain exhibits little regional heterogeneity during ejection throughout the LV wall $[4,18]$. From these observations it was hypothesized that myocytes might adapt orientation to achieve a preferred mechanical load [1].

According to the hypothesis of myofiber reorientation, Rijcken et al. [12] performed a model study where a parameterized distribution of fiber orientations was optimized for minimal heterogeneity in myofiber shortening during ejection. At minimum heterogeneity, fiber orientations were found to compare favorably with experimental data. While it was shown that myofiber orientations can be predicted on the basis of a simple criterion, a global parameterized distribution is not apt to predict local reorientation of myofibers during, e.g., disease. Kroon et al. [9] developed a non-parameterized model in which fiber reorientation was considered as a local response to local fiber cross-fiber shear strain. Their model was also capable of predicting a realistic myofiber structure. During the adaptation process a significant increase in homogenization of mechanical load over the LV wall was obtained and the LV generated more pump work with the same amount of mass.

The aim of this study is to further investigate the computational model by Kroon et al. [9], in particular with respect to the effect of initial fiber orientations and the influence of boundary conditions at the base on long-term outcome of the adaptive process.

\subsection{Methods}

\subsubsection{Modeling myofiber reorientation}

Kroon et al. [9] modeled myofiber reorientation as a response to local loss of myocardial integrity due to forces generated by fiber cross-fiber shear strains 


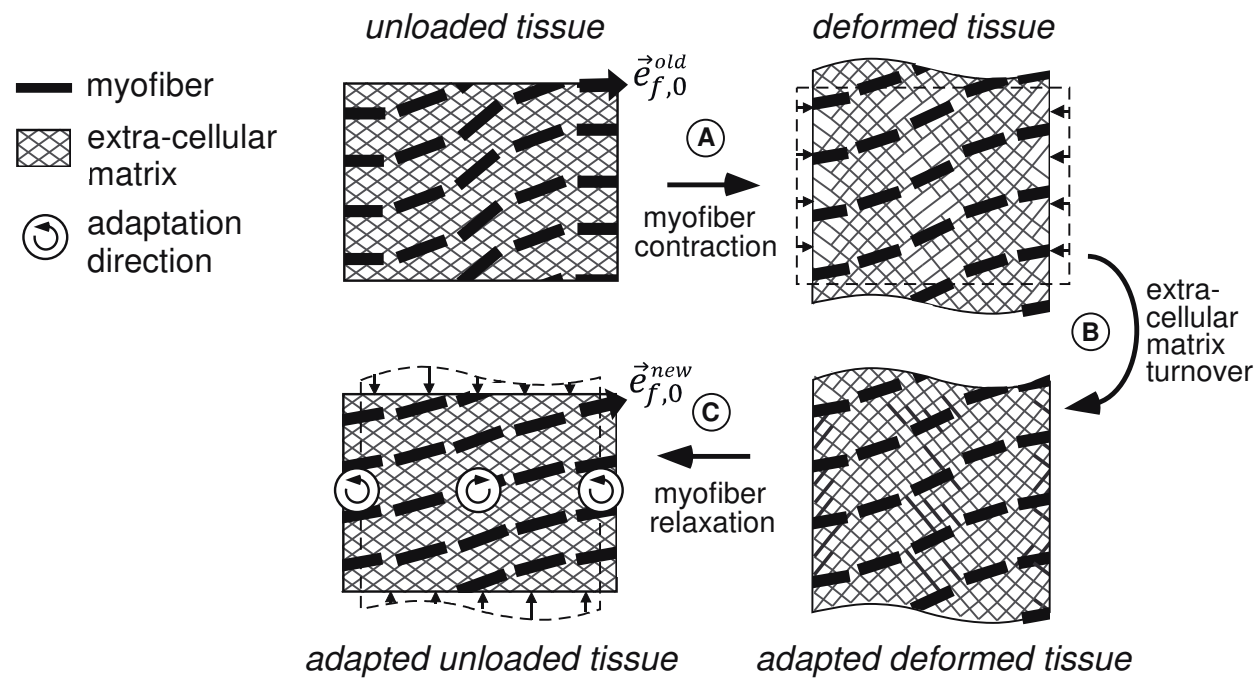

Figure 2.1: Schematic description of the hypothesis on adaptation of myofiber orientation where the old unloaded myofiber direction $\vec{e}_{f, 0}^{\text {old }}$ evolves through $A, B$, and $C$ towards the new adapted unloaded myofiber direction $\vec{e}_{f, 0}^{\text {new }}$ [9].

during myofiber contraction. These shear forces affect connections between extra-cellular matrix (ECM) and myofibers (Fig 2.1). New connections are continuously formed during diastolic and systolic phase of the cardiac cycle. When a certain connection is made, the actual orientation field tends to be fixed within the tissue. The process of breaking and forming of ECM connections itself was not explicitly modeled. Instead, the evolution of the myofiber orientation in the unloaded state $\vec{e}_{f, 0}$ towards the actual myofiber orientation $\vec{e}_{f}$ corrected for rigid body rotation was phenomenologically described by [9]:

$$
\frac{\partial \vec{e}_{f, 0}}{\partial t}=\frac{1}{\kappa}\left(\vec{e}_{f}^{*}-\vec{e}_{f, 0}\right)
$$

with $\kappa$ the adaptation time constant and $\vec{e}_{f}^{*}$ the myofiber direction corrected for rigid body rotations described by:

$$
\vec{e}_{f}^{*}=\frac{\boldsymbol{U} \cdot \vec{e}_{f, 0}}{\lambda_{f}} ; \quad \lambda_{f}=\left|\boldsymbol{U} \cdot \vec{e}_{f, 0}\right|
$$

Here, $\lambda_{f}$ represents the myofiber stretch ratio and $U$ the tensor that describes deformation of the tissue excluding rigid body rotations $R$ according to:

$$
\boldsymbol{U}=\boldsymbol{R}^{-1} \cdot \boldsymbol{F}
$$


with $\boldsymbol{F}$ the deformation gradient tensor. Since rigid body rotations are unlikely to be sensed by the tissue, they are considered irrelevant to adaptation and therefore excluded in evolution Eq 2.1.

Notice that according to Eqs 2.1-2.3, myofiber reorientation only occurs in case neither of the principal strain directions (eigenvectors of $\boldsymbol{U}$ ) coincides with the unloaded fiber direction $\vec{e}_{f, 0}$, i.e., in case of fiber cross-fiber shear. To which of the principal strain directions the fiber orientation ultimately aligns, is not prescribed but follows from the deformation field.

\subsubsection{Modeling cardiac mechanics}

Tissue deformations during the cardiac cycle are calculated with the finite element (FE) model of LV mechanics by Bovendeerd et al. [2], that will only be described in brief.

Geometry and myofiber orientation In the passive stress-free state, a thickwalled geometry is assumed (Fig 2.2). The endocardial and epicardial surfaces are described by truncated ellipsoids. In this state, wall and cavity volumes equal $136 \mathrm{ml}$ and $44 \mathrm{ml}$, respectively. The unloaded geometry remains unaltered during adaptation. Myofiber orientation $\vec{e}_{f, 0}$ is prescribed with respect to the local cardiac coordinate system $\left\{\vec{e}_{l, 0}, \vec{e}_{t, 0}, \vec{e}_{c, 0}\right\}$, where the subscript 0 refers to the unloaded state. The transmural direction $\vec{e}_{t, 0}$ is defined as the outer normal to the cardiac surfaces. The longitudinal direction $\vec{e}_{l, 0}$ is defined perpendicular to $\vec{e}_{t, 0}$ from apex to base. To obtain a righthanded coordinate system, the circumferential direction $\vec{e}_{c, 0}$ is defined in clockwise direction when viewing the LV in apex-to-base direction. Myofiber orientations are described by two angles. The helix angle $\alpha_{h, 0}$ is defined as the angle between $\vec{e}_{c, 0}$ and the projection of $\vec{e}_{f, 0}$ on the circumferential-longitudinal plane $\left(\vec{e}_{c, 0}, \vec{e}_{l, 0}\right)$. The transverse angle $\alpha_{t, 0}$ is defined as the angle between $\vec{e}_{c, 0}$ and the projection of $\vec{e}_{f, 0}$ on the circumferential-transmural plane $\left(\vec{e}_{c, 0}, \vec{e}_{t, 0}\right)$ (Fig 2.2A).

Material properties Myocardial tissue Cauchy stress $\sigma$ is composed of a passive component $\sigma_{p}$ and an active component $\sigma_{a}$ :

$$
\sigma=\sigma_{p}+\sigma_{a} \vec{e}_{f} \vec{e}_{f}
$$

Active stress $\sigma_{a}$ is modeled through a series arrangement of a contractile and a series elastic element. The magnitude of $\sigma_{a}$ depends on time $t_{a}$ elapsed since activation, sarcomere length $l_{s}$, and sarcomere shortening velocity $\partial l_{s} / \partial t$ [8]:

$$
\sigma_{a}=f\left(t_{a}, l_{s},-\frac{\partial l_{s}}{\partial t}\right)
$$


A

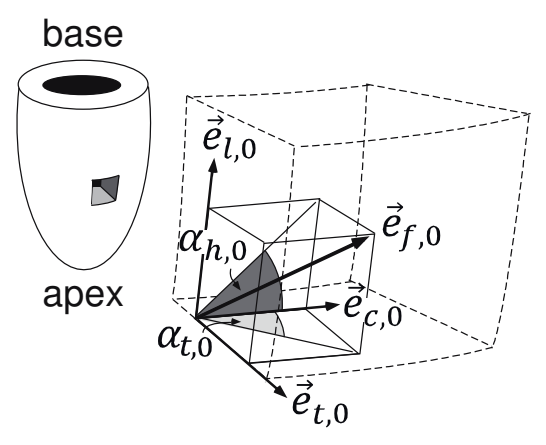

B

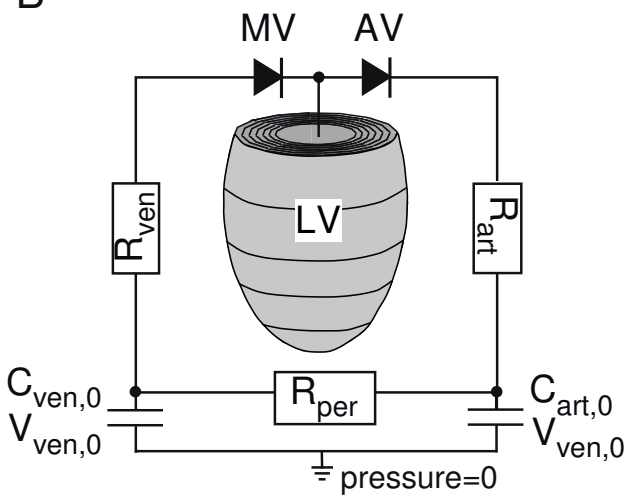

Figure 2.2: Computational model of LV mechanics. A: Ellipsoidally shaped model of LV mechanics showing myofiber orientation vector $\vec{e}_{f, 0}$ described by $\alpha_{h, 0}$ and $\alpha_{t, 0}$ in a local cardiac coordinate system $\left\{\vec{e}_{t, 0}, \vec{e}_{t, 0}, \vec{e}_{c, 0}\right\}$. B: The finite element (FE) mesh consists of 30 elements and is incorporated in a lumped parameter model of the circulation. $\mathrm{AV}$, aortic valve; $C_{\text {art }}$, arterial compliance; $C_{\text {ven }}$, venous compliance; $\mathrm{MV}$, mitral valve; $R_{\text {art }}$, arterial resistance; $R_{\text {per }}$, peripheral resistance; $R_{v e n}$, venous resistance; $V_{\text {art }, 0}$,

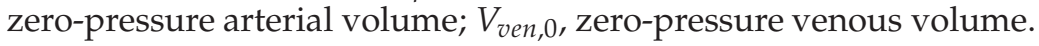

Active stress development is initiated simultaneously with a cycle time of 800 ms. Passive material behavior is assumed nonlinearly elastic, transversely isotropic, and nearly incompressible.

Governing equations and boundary conditions In the model, the equations of conservation of linear momentum are solved:

$$
\vec{\nabla} \cdot \sigma=\overrightarrow{0}
$$

with $\vec{\nabla}$ the spatial gradient operator. At the base, essential boundary conditions are defined to suppress rigid body motion and to represent the mechanical effect of structures left out of the model, e.g., the valvular annulus. The basal boundary conditions are subject of variation in this study (see Section 2.2.3). The epicardial surface is assumed to be traction free while the endocardial surface is uniformly subjected to left ventricular pressure $p_{l v}$. During isovolumic contraction (IC) and relaxation (IR) phases of the cardiac cycle, $p_{l v}$ is determined such that mechanical equilibrium of the myocardial tissue is obtained at a constant end-diastolic or end-systolic left ventricular volume, respectively. During the filling and ejection phase, $p_{l v}$ is computed from the interaction of the LV with the circulation as described in [2]. 
Numerical implementation The equilibrium equations (5.4) are solved numerically with a Galerkin type finite element method using 27-noded hexahedral elements with a tri-quadratic interpolation of the displacement field. The tensor components in (5.4) are described with respect to a cylindrical coordinate system. This allows description of the LV wall with 1 circumferential element, thus reducing computational demand. In total, the LV wall is represented by 30 elements: 6 elements in radial, 1 in circumferential and 5 in longitudinal direction.

\subsubsection{Simulations performed}

Four simulations were performed with combinations of 2 initial conditions of $\alpha_{h, 0}$ and 2 settings for the basal boundary conditions. Initial values of $\alpha_{h, 0}$ were assumed to vary either nonlinearly $(n L I N)$ or linearly $(L I N)$ with the transmural position from endocardium to epicardium. The nonlinear distribution is obtained from [2] (Fig 2.3 dashed lines). The linear distribution varies from $60^{\circ}$ at the endocardium to $-60^{\circ}$ at the epicardium (Fig 2.4 dashed lines in simulations LIN). At the base, axial displacement was suppressed either at the whole basal surface (FULL), or at the endocardial ring only (ENDO). In both cases, circumferential displacement was only suppressed at the endocardial ring.

In all simulations, the first 10 consecutive cardiac cycles were used to reach a hemodynamic steady state and myofiber reorientation was not included. After these 10 cycles myofiber reorientation was simulated over 25 adaptation cycles through the whole LV. Adaptation time constant $\kappa$ was set to $3200 \mathrm{~ms}$. Before reorientation, the transverse angle $\alpha_{t, 0}$ in the reference state was set to zero.

\subsubsection{Postprocessing}

Quantification of adaptation process Evolving fiber orientations, local mechanics, and global hemodynamics are analyzed to asses the effect of the adaptation process on LV structure and function. Baseline values are derived from the hemodynamic steady state after 10 cardiac cycles.

Structural response is quantified by changes in transmural distribution of angles $\alpha_{h, 0}$ and $\alpha_{t, 0}$ in 7 longitudinal levels from apex to base (Fig 2.3 right). The angles are determined from the fiber vectors in a postprocessing step.

The effect of adaptation on local tissue mechanics is quantified by change in mean and standard deviation (SD) of five variables: maximum myofiber stress $\sigma_{f, \max }$, stroke work density $w_{f}$, natural myofiber strain during isovolumic contraction $\epsilon_{f, i c}$, during ejection $\epsilon_{f, e j}$, and during isovolumic relaxation $\epsilon_{f, i r}$. The $w_{f}$ at each point is defined as the area enclosed by the myofiber 
stress-natural strain loop:

$$
w_{f}=\oint \sigma_{f} d \epsilon_{f} ; \quad \epsilon_{f}=\ln \lambda_{f}
$$

Evolution of homogeneity is quantified by the average of the SDs of strains $\epsilon_{f, i c}, \epsilon_{f, e j}$, and $\epsilon_{f, i r}$ :

$$
S \bar{D}_{\epsilon_{f}}=\frac{S D_{\epsilon_{f, i c}}+S D_{\epsilon_{f, e j}}+S D_{\epsilon_{f, i r}}}{3}
$$

with $S D_{\epsilon_{f, i c}} S D_{\epsilon_{f, e j}} S D_{\epsilon_{f, i r}}$ the SDs of $\epsilon_{f, i c}, \epsilon_{f, e j}$, and $\epsilon_{f, i r}$, respectively. Evolution of the SD of $\sigma_{f, \max }\left(S D_{\sigma}\right)$ is analyzed too.

Global cardiac function is quantified by maximum LV pressure $p_{l v, \max }$, stroke volume $S V$ and stroke work $W$ given by the area enclosed by the LV pressure-volume loop:

$$
W=\oint p_{l v} d V_{l v}
$$

Comparison with experimental data Left ventricular circumferential-radial shear $E_{c r}$ and torsion $\tau$ as computed with the model before and after myofiber reorientation are compared with those determined from magnetic resonance tagging (MRT) experiments. The protocol for the MRT measurements and determination of the Green-Lagrange strain tensor component $E_{c r}$ have been described previously in [2] and [16]. Definition of $\tau$ has been described previously in [5]. Essentially, $\tau$ quantifies the base-to-apex gradient of rotation about the LV long axis. $E_{c r}$ quantifies the endo-to-epi gradient of this rotation. In the model, $E_{c r}$ and $\tau$ are determined at the levels of the MR slices by interpolation from adjacent nodal points in the FE mesh. The locations of the MR slices relative to the model geometry are shown in Fig 2.8 on the top left. In agreement with the experimental procedure, strains are computed with respect to begin-ejection and averaged in radial direction.

\subsection{Results}

Changes in transmural distribution of myofiber angles $\alpha_{h, 0}$ and $\alpha_{t, 0}$ in simulation FULL+nLIN are shown in Fig 2.3. Within 1 adaptation cycle, a gradient for $\alpha_{t, 0}$ develops with negative values at the apex and positive values at the base. Largest amplitudes develop within 5 adaptation cycles. Thereafter, the location of the extrema shifts from endocardium towards mid-wall. At the apical levels the amplitude decreases. As adaptation proceeds, endocardial $\alpha_{h, 0}\left(\alpha_{h, 0}^{\text {endo }}\right)$ in the upper half of the LV wall evolves towards $0^{\circ}$ (circumferentially oriented myofibers), whereas epicardial $\alpha_{h, 0}\left(\alpha_{h, 0}^{e p i}\right)$ evolves towards $-90^{\circ}$ (axially oriented myofibers). 


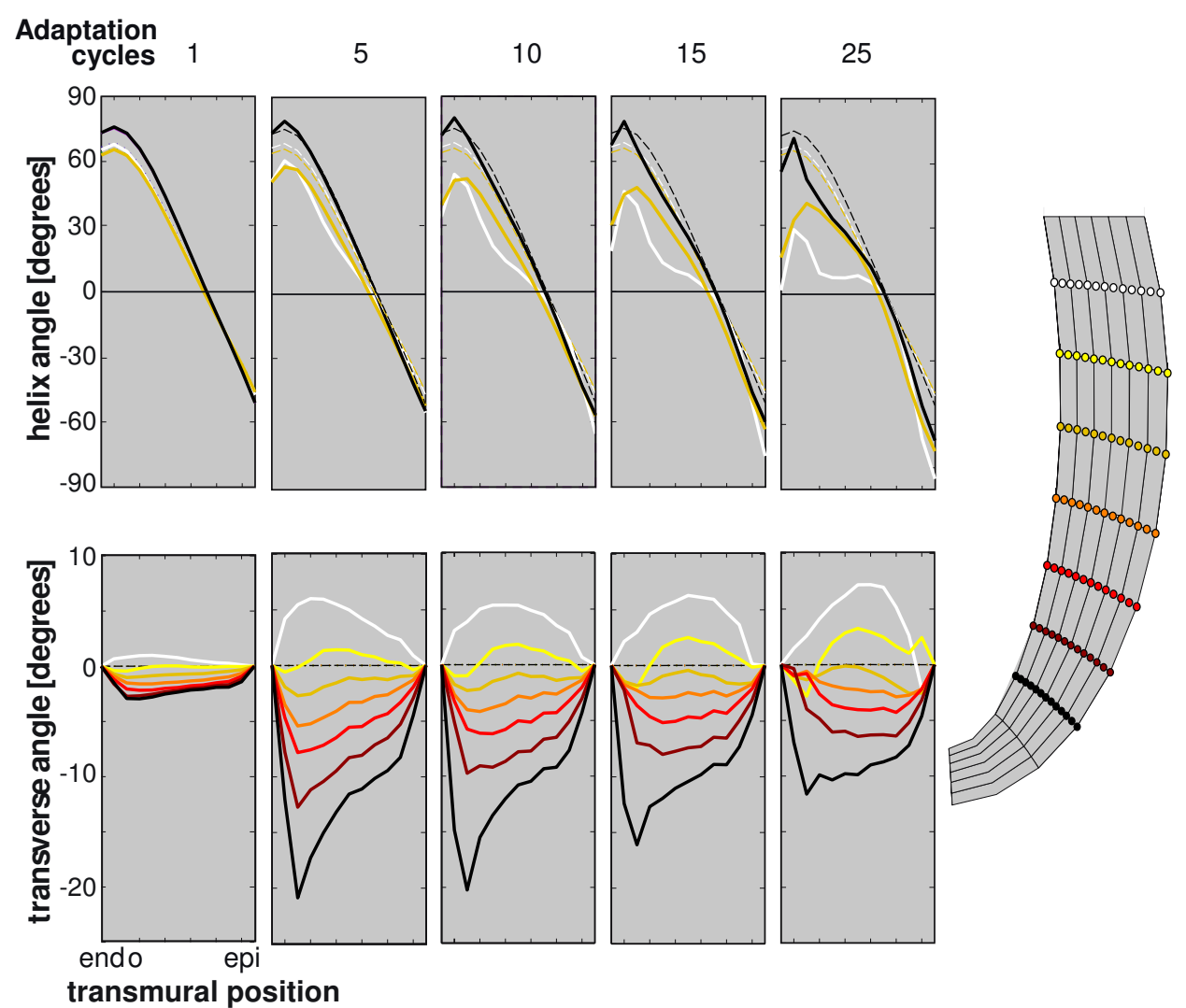

Figure 2.3: Simulation FULL+nLIN. Development of helix angle $\alpha_{h, 0}$ (top) and transverse angle $\alpha_{t, 0}$ (bottom) after 1, 5, 10,15, and 25 adaptation cycles. Transmural course in myofiber angles before (- $)$ and after (-) myofiber adaptation is shown for 7 longitudinal levels in the LV wall (right). 


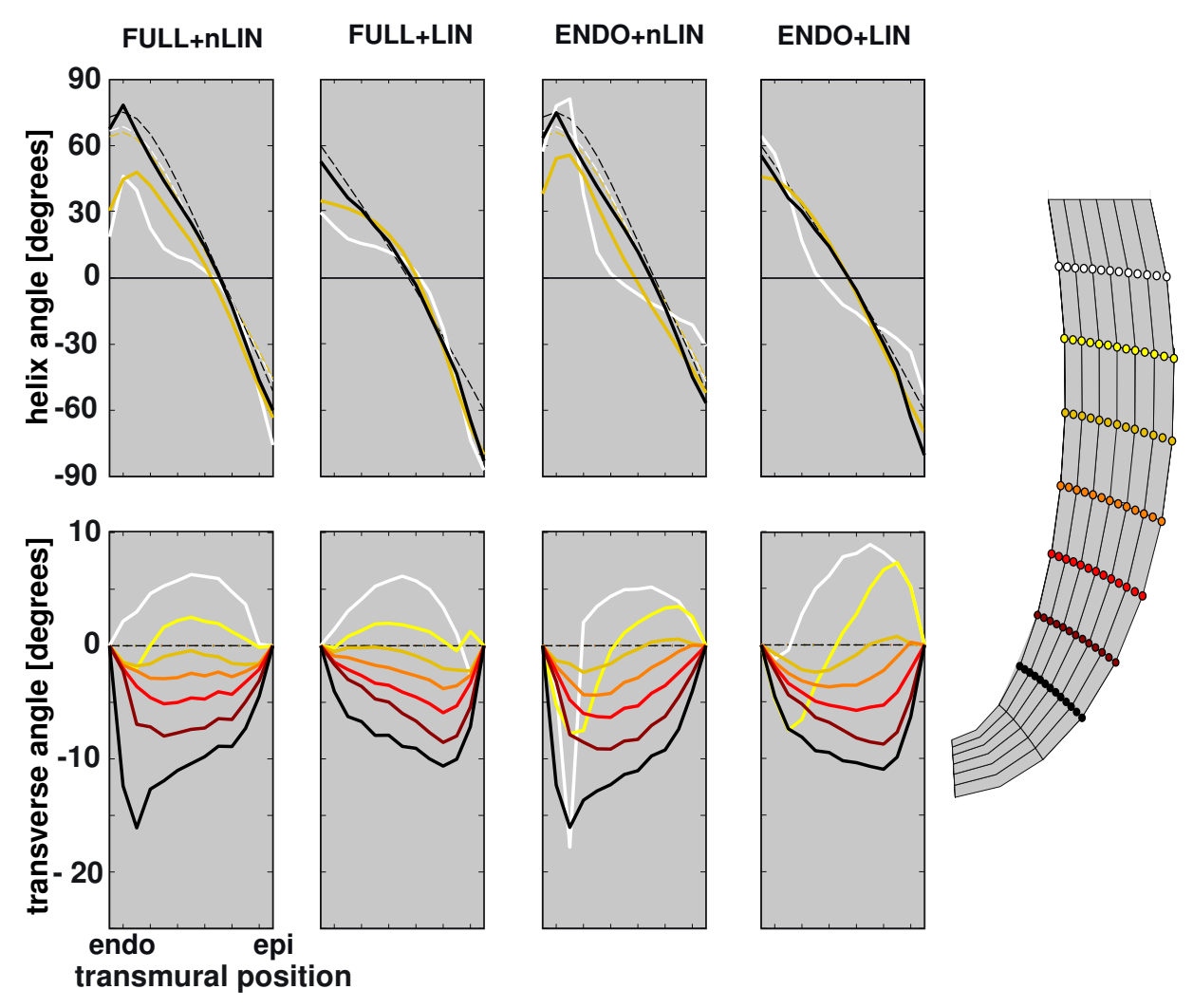

Figure 2.4: Transmural course in helix angle $\alpha_{h, 0}$ (top) and transverse angle $\alpha_{t, 0}$ (bottom) in all simulations before adaptation (- -) and after 15 adaptation cycles (-) at the longitudinal levels shown in Fig 2.3. 
In Fig 2.4, transmural distributions of $\alpha_{h, 0}$ and $\alpha_{t, 0}$ are shown for all simulations after 15 adaptation cycles. The fast response of $\alpha_{t, 0}$ and slow response of $\alpha_{h, 0}$ was observed in the other simulations too. After 15 adaptation cycles, the effect of the initial condition of $\alpha_{h, 0}$ (LIN vs. $\left.n L I N\right)$ was found to be reduced, while the effect of the basal boundary conditions (FULL vs. ENDO) persisted. As in FULL+nLIN, $\alpha_{h, 0}^{e n d o}$ evolves towards $0^{\circ}$ and $\alpha_{h, 0}^{e p i}$ towards $-90^{\circ}$ in $F U L L+L I N$. For $E N D O+n L I N$ and $E N D O+L I N$ the opposite can be observed. In these simulations $\alpha_{h, 0}^{\text {endo }}$ evolves towards $90^{\circ}$ (axial myofiber orientation) and $\alpha_{h, 0}^{e p i}$ towards $0^{\circ}$ (circumferential orientation). A similar gradient for $\alpha_{t, 0}$ develops in all simulations. Near the base, $\alpha_{t, 0}^{\text {endo }}$ is positive for FULL+nLIN and FULL+LIN, whereas $\alpha_{t, 0}^{\text {endo }}$ is negative for ENDO+nLIN and ENDO+LIN.

In all simulations, local and global cardiac function increase significantly during the first adaptation cycles as indicated by the increase in maximum myofiber stress $\sigma_{f, \max }$, stroke work density $w_{f}$, myofiber shortening (decrease of $\left.\epsilon_{f, e j}\right)$, maximum left ventricular pressure $p_{l v}$, stroke volume $S V$, and stroke work $W$. In addition, fiber strains during the isovolumic phases, $\epsilon_{f, i c}$ and $\epsilon_{f, i r}$, decrease significantly. Detailed results of simulation FULL+nLIN are shown in Fig 2.5. For all simulations, evolution of homogeneity is indicated in Fig 2.6 where development of $S \bar{D}_{\epsilon_{f}}$ and $S D_{\sigma}$ are shown. Here, a fast response during the first adaptation cycles is observed too. All simulations show an increase in homogeneity of average strain during the first adaptation cycles. Strain homogeneity increases further in simulations LIN, while it decreases in simulations $n L I N$. During the whole adaptation process, homogeneity of $\sigma_{f, \max }$ increases for all simulations except for ENDO+nLIN. In ENDO+nLIN, both $S \bar{D}_{\epsilon_{f}}$ and $S D_{\sigma}$ reach a minimum after 2 adaptation cycles. Finally, $S \bar{D}_{\epsilon_{f}}$ and $S D_{\sigma}$ evolve to 2 values, solely dependent on the basal boundary conditions.

For all simulations, the model predicts fiber structures with distributions of $\alpha_{h, 0}$ and $\alpha_{t, 0}$ within the, admittedly wide, range of experimental data (Fig 2.7). Furthermore, patterns of circumferential-radial shear $E_{c r}$ are more realistic after myofiber reorientation (Fig 2.8). $E_{c r}$ amplitudes reduce from $\sim 0.35$ before to $\sim 0.18$ after reorientation, while experimental amplitudes of $\sim 0.1$ are observed. Before adaptation, the axial gradient of $E_{c r}$ at end ejection is opposite to experimental observations. After adaptation, the gradient flips and becomes similar to the experiment. Before adaptation, two characteristic patterns of torsion $\tau$ can be observed determined by the initial conditions of $\alpha_{h, 0}$ (LIN vs. $n L I N)$. Differences in $\tau$ disappear after adaptation. Minimum $\tau$ is reached before end of ejection, which is early when compared with experimental $\tau$. The return to zero of $\tau$ occurs slower in the model than in the experiment. 

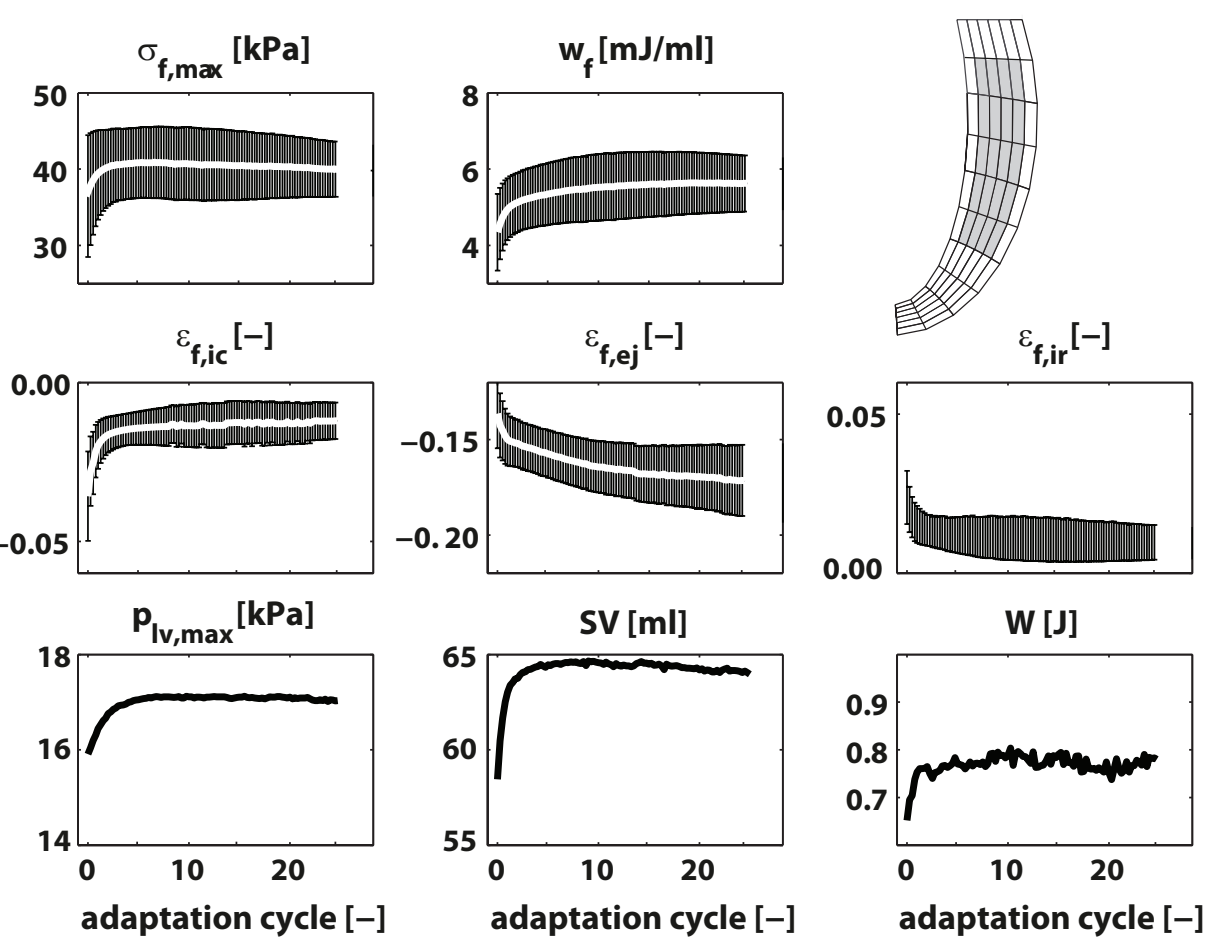

Figure 2.5: Evolution of local and global function in FULL+nLIN during 25 adaptation cycles. Adaptation cycle is defined as time $t$ normalized with respect to adaptation time constant $\kappa(t / \kappa)$. Local function is presented by means and standard deviations calculated from the grey area indicated in the geometry (top right) of maximum myofiber stress $\sigma_{f, \text { max }}$, stroke work density $w_{f}$ (top row), natural myofiber strain during isovolumic contraction $\epsilon_{f, i c}$, during ejection $\epsilon_{f, e j}$, and during isovolumic relaxation $\epsilon_{f, i r}$ (middle row). Global function is presented by maximum LV pressure $p_{l v, \text { max }}$ stroke volume $S V$, and stroke work $W$ (bottom row). 
$\overline{\mathbf{S D}}_{\varepsilon_{f}}[-]$

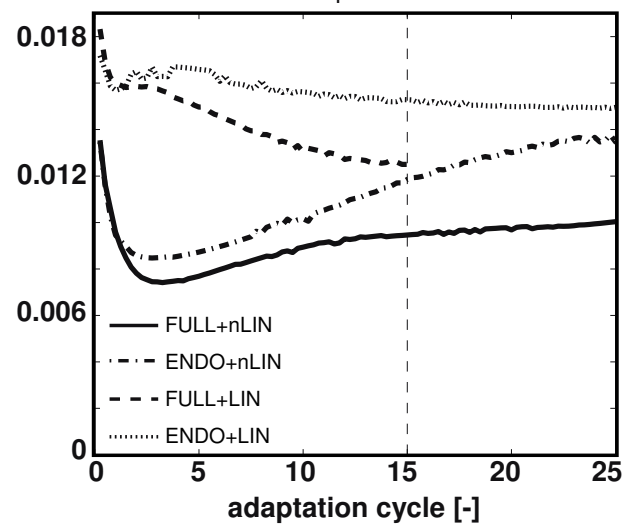

$\mathrm{SD}_{\sigma_{f}}[\mathrm{kPa}]$

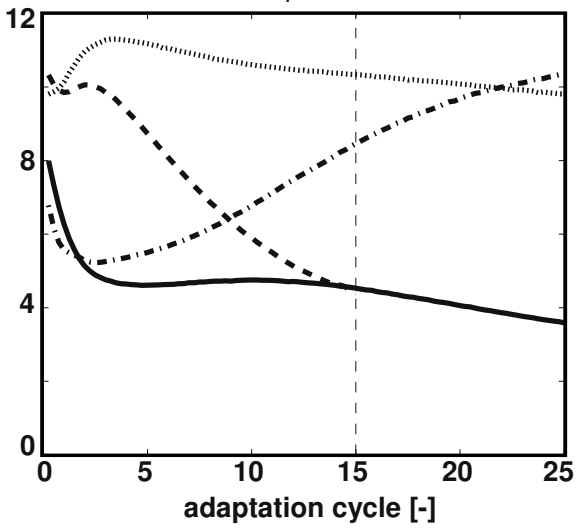

Figure 2.6: Evolution of average standard deviation (SD) of strains $\left(S \bar{D}_{\epsilon_{f}}\right.$, left) and SD of maximum myofiber stress $\sigma_{f, \max }\left(S D_{\sigma_{f}}\right.$, right) in simulations FULL+nLIN (-),

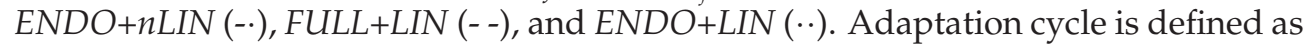
time $t$ normalized with respect to adaptation time constant $\kappa(t / \kappa)$. Results in Fig 2.4 are presented after 15 adaptation cycles.

\subsection{Discussion}

In this study, we further investigated a computational model in which left ventricular (LV) myofiber reorientation was considered as a local response to local fiber cross-fiber shear strain [9]. The effect of the initial fiber orientations, and the influence of the boundary conditions at the base on the long-term outcome of the adaptive process were investigated.

In all simulations a fast development of transverse angle $\alpha_{t, 0}$ and a slow response of helix angle $\alpha_{h, 0}$ was observed. This is presumably caused by the fact that the initial $\alpha_{h, 0}$ was chosen in the range of experimental data, while $\alpha_{t, 0}$ was set to an unphysiological value of zero. From the transmural distributions of fiber angles (Fig 2.4) and from the evolution of homogeneity in stress and strain (Fig 2.6), it was observed that the simulations with the same boundary condition showed a similar behavior during adaptation that was irrespective of the initial fiber orientation. The effect of the initial condition of $\alpha_{h, 0}$ on especially homogeneity diminishes during adaptation: simulations where boundary conditions were applied at the whole basal surface (FULL) evolved to similar SD values of (Fig 2.6). So did the simulations where boundary conditions were applied at the endocardial basal ring (ENDO). This suggests that one single optimal fiber orientation exists, which agrees with observations in optimization studies [12,17].

The basal boundary conditions had a significant effect on the adaptation process. Initially, the basal boundary conditions affect deformation near the 
base only. This deformation pattern affects fiber reorientation in the basal region, which in turn affects deformation also in regions closer to the equator. Thus, basal boundary conditions gradually affects fiber orientation in the complete LV (Fig 2.3).

To enforce boundary conditions, external forces are implicitly applied. Fibers near the base tend to align in the direction of these forces. Consequently, in simulations FULL fibers oriented perpendicular to the epicardial base $\left(\alpha_{h, 0}\right.$ towards $\left.-90^{\circ}\right)$, whereas in simulations $E N D O$, they oriented towards the endocardial basal ring only (negative $\alpha_{t, 0}, \alpha_{h, 0}$ towards $90^{\circ}$, Fig 2.4). The latter pattern is more in agreement with anatomical data. However, considering the amplitude of radial-axial shear strain $E_{r z}$ near the base, values in simulations FULL $(\sim 0.08)$ are closer to experimental values $(\sim 0.02,[20])$ than those in simulations $E N D O(\sim 0.20)$, suggesting that the boundary conditions in simulations FULL are more realistic. Prescribing boundary conditions in terms of forces instead of displacements, i.e., allowing limited axial basal movement, might be a more realistic way for modeling boundary conditions at the base [14].

A decrease of myofiber strain during the isovolumic phases $\left(\epsilon_{f, i c}\right.$ and $\left.\epsilon_{f, i r}\right)$ was observed in all simulations and indicated that differences between fiber orientations in the loaded and unloaded tissue indeed decreased. Thus, fiber cross-fiber shear minimized as a result of deformation-induced reorientation. Fiber directions evolved towards a principal strain direction that was not prescribed in Eq 2.1. At begin ejection, we found that the minimal stretch direction was oriented perpendicular to the LV wall. Consequently, intermediate and maximum stretch direction are oriented within the plane of the wall. It turned out that fiber directions evolved towards the intermediate stretch direction, in agreement with the expectation that active fibers generate a compressive force on the tissue along their direction.

Comparison with experimental data From the comparison with experimental data (Fig 2.7), no model can be preferred since predicted values of $\alpha_{h, 0}$ and mid-wall $\alpha_{t, 0}$ were quite similar for all simulations. However, restriction to presentation of mid-wall $\alpha_{t, 0}$ neglects the transmural differences which were clearly visible in Fig 2.4. Since no experimental data on the transmural variation of the transverse angle exists, the model could not be verified in this respect.

The transmural distribution of the transverse angle is important since it determines the pattern of circumferential-radial shear $E_{c r}$ [2]. $E_{c r}$ patterns were more realistic after myofiber reorientation in all simulations (Fig 2.8). The improved agreement between $E_{c r}$ in simulation FULL+nLIN and experiment was previously presented by Kroon et al. [10], and is in line with the 

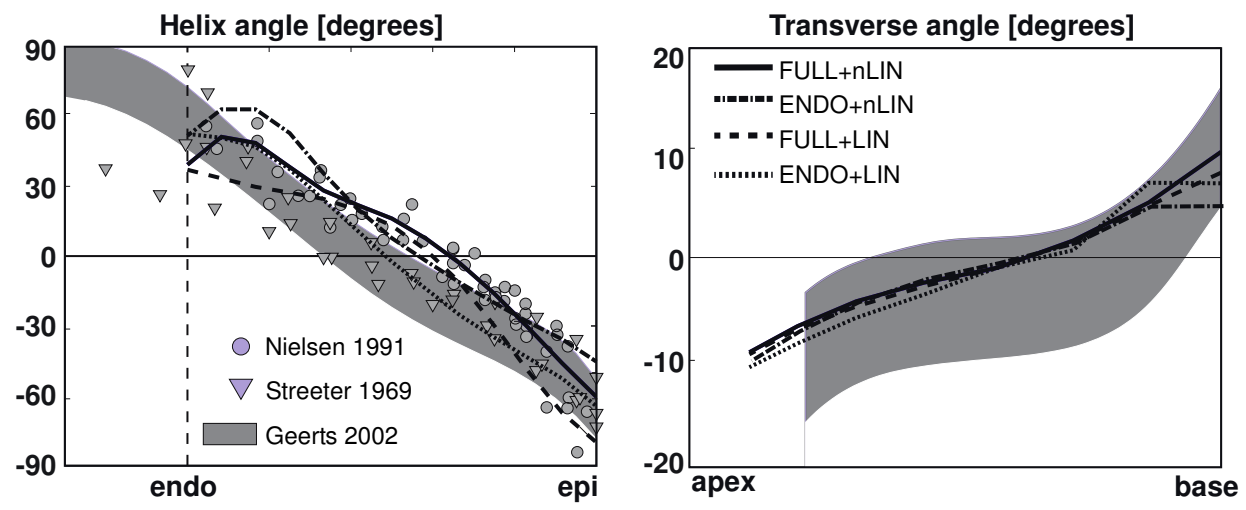

Figure 2.7: Comparison of predicted myocardial structure after 15 adaptation cycles with experimental data $[6,11,15]$. Left : Helix angle $\alpha_{h, 0}$ at equatorial level. Right : Transverse angle $\alpha_{t, 0}$ at mid-wall. The mid-wall region was defined as the transmural region with an absolute helix angle value of less than $5^{\circ}$.

study by Bovendeerd et al. [2], where it was concluded that agreement with experimental data could only be obtained when a non-zero $\alpha_{t, 0}$ was included. Ubbink et al. [16] demonstrated a high sensitivity of $E_{c r}$ on $\alpha_{t, 0}$. Differences in transmural variation of $\alpha_{t, 0}$ might therefore explain the differences in $E_{c r}$ patterns between simulations.

Before reorientation, two torsion patterns can be distinguished (Fig 2.8). Since $\alpha_{t, 0}$ was set to zero in all simulations, the differences might be a result of the differences in $\alpha_{h, 0}$. After reorientation, $\tau$-patterns were similar, despite differences in $\alpha_{h, 0}$ and $\alpha_{t, 0}$. The early appearance of the minimum in $\tau$ and the slow return to zero as compared to experiments, presumably were a result of the choice of the time constants for onset and decay of active stress. Furthermore, the 'hump' in the $\tau$-pattern early in the filling phase, was found to be the result of inhomogeneous relaxation. In the model, sarcomere length was assumed homogeneous in the unloaded LV. As a result, sarcomere length is inhomogeneous at begin contraction. Since both the level of actively generated myofiber stress and the duration of the twitch are directly related to sarcomere length, relaxation is inhomogeneous. In a simulation where sarcomere length was chosen homogeneous at begin contraction, relaxation was homogeneous and the 'hump' disappeared.

Study assumptions and model limitations The model presented only accounts for myofiber reorientation. Other adaptation mechanisms are likely to be active as well. Clinically, one of the most evident examples of adaptation is the change in LV wall mass and cavity volume in response to pressure and volume overload, respectively. In addition, in reality the externally un- 


\section{Before reorientation}
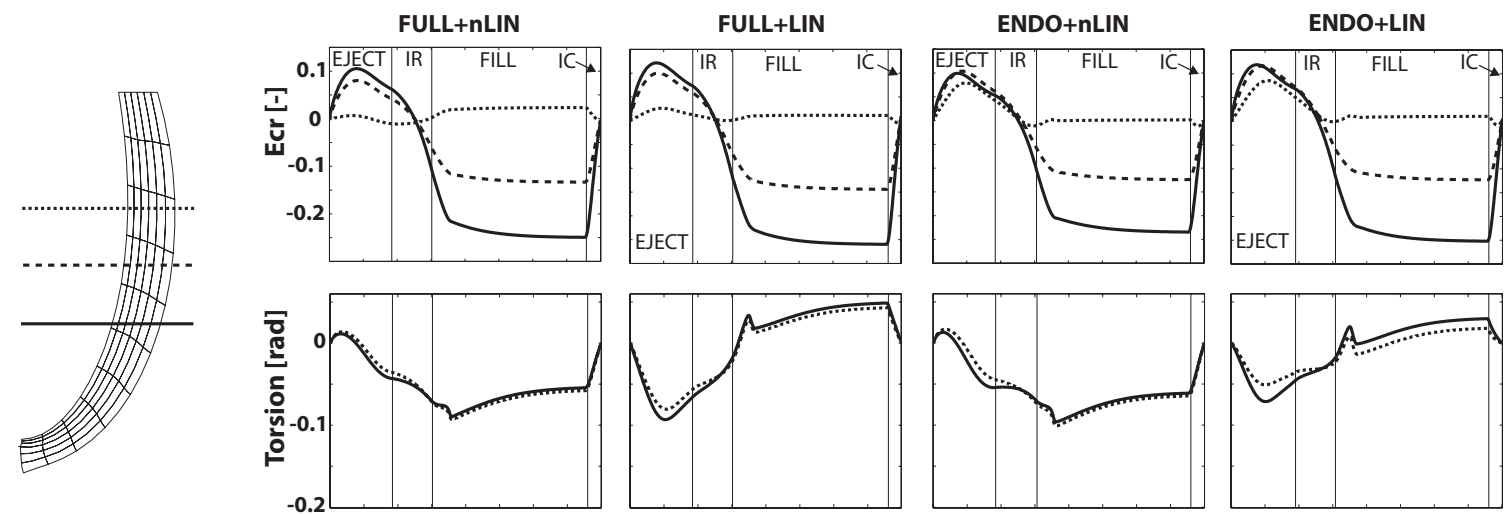

Experimental data
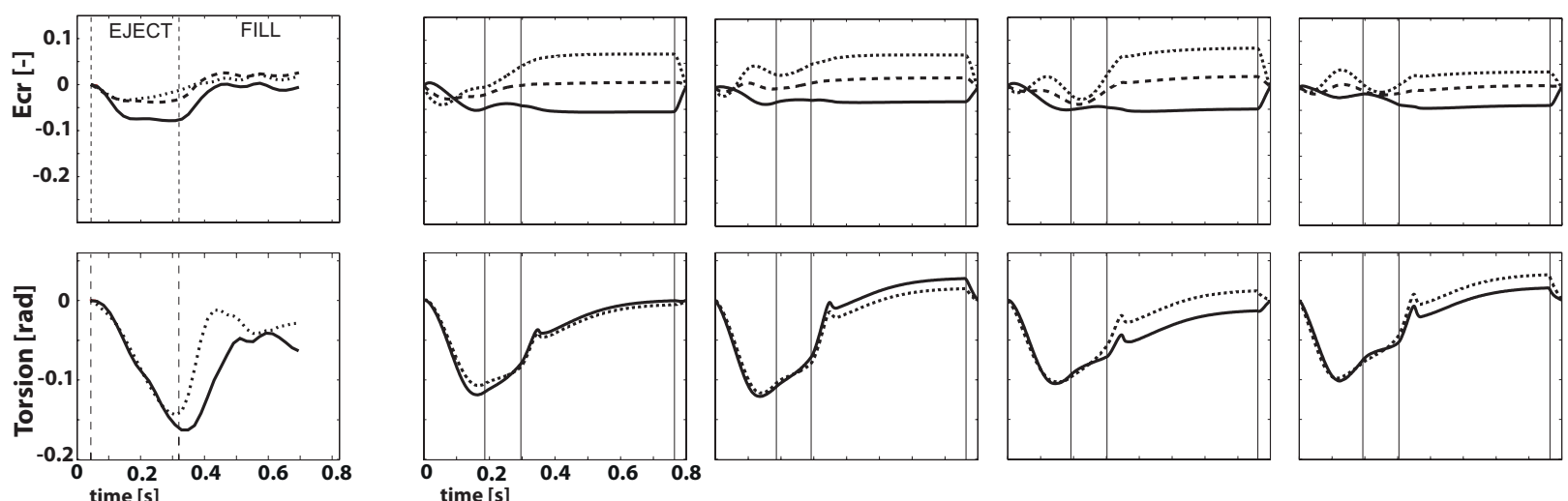

Figure 2.8: Model computed circumferential-radial shear $E_{c r}$ and torsion $\tau$ before adaptation (top) and after 15 adaptation cycles (bottom). On the top left, the LV geometry in simulation FULL+nLIN at begin ejection is shown with the position of the 3 short axis slices relative to the geometry. On the bottom left, a typical example of experimental data is presented [2]. EJECT=ejection phase; $I R=$ isovolumic relaxation phase; $F I L L=$ filling phase; $I C=$ isovolumic contraction phase. 
loaded LV exhibits a transmural gradient in sarcomere length with epicardial sarcomeres being longer than endocardial ones [13]. This might be a result of mechanically induced adaptation as well. Extension of the model with these adaptive mechanisms should be considered.

\subsection{Conclusion}

In this study, we further investigated a computational model of shear-induced myofiber reorientation in the left ventricular wall. It is concluded that the choice of boundary conditions at the base influences long-term development of fiber orientation while the effect of initial setting of fiber orientation field disappears. Thus, for modeling adaptive reorientation of myofibers, physiological boundary conditions at the base are relevant. Due to large spread in experimental data on fiber orientation and the lack of detailed data on the transverse angle at the base, no clear conclusion can be drawn with respect to which of the boundary conditions is preferable. After reorientation, differences between model predicted and experimental observation of LV structure and deformation were reduced significantly, but a complete match could not be obtained. 


\section{References}

1. Arts T, Prinzen FW, Snoeckx LH, Rijcken JM, and Reneman RS (1994). "Adaptation of cardiac structure by mechanical feedback in the environment of the cell: a model study". Biophysical Journal 66, pp. 953-961.

2. Bovendeerd PHM, Kroon W, and Delhaas T (2009). "Determinants of left ventricular shear strain". Am J Physiol - Heart Circ Physiol 297.3, H1058-H1068.

3. Costa KD, Holmes JW, and McCulloch AD (2001). "Modelling cardiac mechanical properties in three dimensions". Phil Trans R Soc Lond A 359, pp. 1233-1250.

4. Delhaas T, Arts T, Bovendeerd P, Prinzen F, and Reneman R (1993). “Subepicardial fiber strain and stress as related to left ventricular pressure and volume". Am J Physiol - Heart Circ Physiol 264, H1548-H1559.

5. Delhaas T, Kroon W, Decaluwe W, Rubbens M, Bovendeerd P, and Arts T (2008). "Structure and torsion of the normal and situs inversus totalis cardiac left ventricle; Part I: experimental data in humans". Am J Physiol - Heart Circ Physiol 295, H197-H201.

6. Geerts-Ossevoort L, Bovendeerd P, Nicolay K, and Arts T (2002). “Characterization of the normal cardiac myofiber field in goat measured with MR-diffusion tensor imaging". Am J Physiol - Heart Circ Physiol 283.1, H139-H145.

7. Hunter PJ, Coveney PV, De Bono B, Diaz V, Fenner J, Frangi AF, Harris P, Hose R, Kohl P, Lawford P, et al. (2010). "A vision and strategy for the virtual physiological human in 2010 and beyond". Phil Trans R Soc Lond A 368, pp. 2595-2614.

8. Kerckhoffs RCP, Bovendeerd PHM, Kotte JCS, Prinzen FW, Smits K, and Arts T (2003). "Homogeneity of cardiac contraction despite physiological asynchrony of depolarization: a model study." Ann Biomed Eng 31.5, pp. 536-547.

9. Kroon W, Delhaas T, Arts T, and Bovendeerd P (2009a). "Computational analysis of the myocardial structure: Adaptation of myofiber orientations through deformation in three dimensions". Med Imag Anal 13, pp. 346-353.

10. Kroon W, Delhaas T, Bovendeerd P, and Arts T (2009b). "Adaptive Reorientation of Cardiac Myofibers: Comparison of Left Ventricular Shear in Model and Experiment". In: Functional Imaging and Modeling of the Heart. Ed. by N Ayache, H Delingette, and M Sermesant. Vol. 5528. Lecture Notes in Computer Science. Springer Berlin Heidelberg, pp. 58-67.

11. Nielsen PM, LeGrice IJ, Smaill BH, and Hunter PJ (1991). "Mathematical model of geometry and fibrous structure of the heart". Am J Physiol - Heart Circ Physiol 260, H1365-H1378.

12. Rijcken JM, Bovendeerd PHM, Schoofs AJG, Van Campen DH, and Arts T (1999). “Optimization of cardiac fiber orientation for homogeneous fiber strain during ejection". Ann Biomed Eng 27, pp. 289-297.

13. Rodriguez EK, Omens JH, Waldman LK, and McCulloch AD (1993). "Effect of residual stress on transmural sarcomere length distributions in rat left ventricle". Am J Physiol - Heart Circ Physiol 264, H1048-H1056. 
14. Saint-Marie J, Chapelle D, Cimrman R, and Sorine M (2006). "Modeling and estimation of the cardiac electromechanical activity". Comp and Struc 84, pp. 1743-1759.

15. Streeter Jr D, Spotnitz HM, Patel DP, Ross Jr J, and Sonnenblick EH (1969). "Fiber orientation in the canine left ventricle during diastole and systole." Circ Res 24.3, pp. 339-347.

16. Ubbink SWJ, Bovendeerd PHM, Delhaas T, Arts T, and Van de Vosse FN (2006). “Towards model-based analysis of cardiac MR tagging data: Relation between left ventricular shear strain and myofiber orientation". Med Imag Anal 10, pp. 623-641.

17. Vendelin M, Bovendeerd PHM, Engelbrecht J, and Arts T (2002). “Optimizing ventricular fibers: uniform strain of stress, but not ATP consumption, leads to high efficiency". Am J Physiol - Heart Circ Physiol 283, H1072-H1081.

18. Waldman LK, Nosan D, Villarreal F, and Covell JW (1988). "Relation between transmural deformation and local myofiber direction in canine left ventricle". Circ Res 63, pp. 550-562.

19. Weis SM, Emery JL, Becker KD, McBride Jr. DJ, Omens JH, and McCulloch AD (2000). "Myocardial mechanics and collagen structure in the osteogenesis imperfecta murine (oim)". Circ Res 87, pp. 663-669.

20. Young A, Kramer C, Ferrari V, Axel L, and Reichek N (1994). “Three-dimensional left ventricular deformation in hypertrophic cardiomyopathy". Circulation 90, pp. 854-867.

21. Zimmerman SD, Karlon WJ, Holmes JW, Omens JH, and Covell JW (2000). "Structural and mechanical factors influencing infarct scar collagen organization". Am J Physiol - Heart Circ Physiol 278, H194-H200. 
Chapter 2 


\section{Chapter 3}

\section{Myofiber reorientation in the situs inversus totalis left ventricle}

The contents of this chapter are based on:

M. Pluijmert, W. Kroon, A.C. Rossi, P.H.M. Bovendeerd, T. Delhaas, Why SIT Works: Normal Function Despite Typical Myofiber Pattern in Situs Inversus Totalis (SIT) Hearts Derived by Shear-induced Myofiber Reorientation, PLoS Computational Biology, 8(7):e1002611, 2012 


\section{Abstract}

The left ventricle (LV) of mammals with Situs Solitus (SS, normal organ arrangement) displays hardly any interindividual variation in myofiber pattern and experimentally determined torsion. SS LV myofiber pattern has been suggested to result from adaptive myofiber reorientation, in turn leading to efficient pump and myofiber function. Limited data from the Situs Inversus Totalis (SIT, a complete mirror image of organ anatomy and position) LV demonstrated an essential different myofiber pattern, being normal at the apex but mirrored at the base. Considerable differences in torsion patterns in between human SIT LVs even suggest variation in myofiber pattern among SIT LVs themselves. We addressed whether different myofiber patterns in the SIT LV can be predicted by adaptive myofiber reorientation and whether they yield similar pump and myofiber function as in the SS LV. With a mathematical model of LV mechanics including shear induced myofiber reorientation, we predicted myofiber patterns of one SS and three different SIT LVs. Initial conditions for SIT were based on scarce information on the helix angle. The transverse angle was set to zero. During reorientation, a non-zero transverse angle developed, pump function increased, and myofiber function increased and became more homogeneous. Three continuous SIT structures emerged with a different location of transition between normal and mirrored myofiber orientation pattern. Predicted SIT torsion patterns matched experimentally determined ones. Pump and myofiber function in SIT and SS LVs are similar, despite essential differences in myocardial structure. SS and SIT LV structure and function may originate from same processes of adaptive myofiber reorientation. 


\subsection{Introduction}

The myofiber orientation pattern in the cardiac left ventricular wall has an invariant nature among mammals, including humans, with a normal organ arrangement (Situs Solitus, SS) [14, 15, 25, 33]. Myofibers follow a left-handed helical path near the epicardium and gradually change their pitch through a circumferential path in the midventricular wall towards a right-handed helical path near the endocardium (figure 3.1, left). The transmural change in helix angle $\alpha_{h}$ is qualitatively the same from apex to base. Moreover, myofibers cross over between endo- and epicardium. The direction of crossover gradually changes from apex to base [11] and is quantified by the transverse angle $\alpha_{t}$.

SS LVs not only display an invariant myofiber pattern, but also a large similarity in experimentally determined measures of deformation, such as torsion $[8,34]$. Contraction of sub-endocardial myofibers with a right-handed helical orientation tends to rotate the apex in a clockwise direction with respect to the base, when viewed from the apex (figure 3.2C). The opposite is true for the sub-epicardium: contraction of myofibers with a left-handed helical orientation, tends to rotate the apex in counterclockwise direction during myofiber contraction. A net counterclockwise rotation of the apex as obtained from measurements [8], indicate that epicardial myofibers dominate endocardial myofibers (figure 3.2D).

Several model studies demonstrated that myofiber orientation pattern is a major determinant of strain distribution in the cardiac wall $[4,6,34]$. In addition, magnetic resonance tagging (MRT) studies showed that myofiber shortening during ejection exhibits little heterogeneity [23]. Even more so, when coefficients of a polynomial that described the spatial distribution of myofiber orientations were optimized for minimal heterogeneity in myofiber shortening during ejection, realistic myofiber orientations were found [29]. Consequently, it was hypothesized that reorientation is an important adaptive mechanism for a myocyte to achieve a preferred mechanical loading state. Indeed, in a computational model of shear-induced adaptive myofiber reorientation, global LV pump as well as local myofiber function increased upon reorientation, while the latter displayed less spatial heterogeneity [19]. This suggests that the invariant nature of myofiber orientation in the SS LV reflects the unique solution of a successful adaptation process at myocyte level.

Scarce experimental and anatomical studies demonstrated that in individuals with Situs Inversus Totalis (SIT), i.e., a complete mirror image of their organ anatomy and position, the myofiber orientation pattern of the $\mathrm{LV}$ is not a complete mirror image of the pattern in the SS LV [3, 7, 8, 24]. Instead, in SIT LVs the transmural change of $\alpha_{h}$ at the apex is as in the SS LV but it changes to a (partially) mirror-imaged transmural distribution at the base $[3,8,24]$ (fig- 
ure 3.1, right). Anatomical data suggest that the transition between the two distributions seems to be located more apically at the endocardium than at the epicardium [3,24], but detailed information is lacking. In addition, no quantitative data on the transmural course of $\alpha_{t}$ have yet been obtained. As can be expected considering the dependency of cardiac deformation on myofiber orientation pattern, torsion in the SIT LV was found to differ from that in the SS $\mathrm{LV}$. At the apex, torsion patterns of SS and SIT coincide, whereas at the base an inverted torsion pattern is observed in SIT when compared to SS (figure 3.2E). More interestingly, torsion patterns have been shown to differ considerably in between SIT LVs [8]. This suggests that myofiber orientations of the SIT $\mathrm{LV}$ not only deviate from that in the SS LV, but also display variation among SIT LVs themselves. Assuming that myocytes in the SIT LV have a normal adaptive response through reorientation, this adaptation process seems to result in multiple outcomes. Despite differences in deformation (and structure), none of the subjects in the SIT group studied by Delhaas et al. [8] showed any cardiac complaints.

In this study, we addressed the question whether variations in myofiber patterns of the SIT LV can be predicted by adaptive reorientation of myofibers, and whether these various outcomes yield similar pump and myofiber function as in the SS LV. To investigate this, we employ a mathematical model of LV mechanics [4] (figure 3.3) and include shear-induced adaptive myofiber reorientation [19]. In the latter model, we assume myofibers to adapt their orientation as a response to local loss of myocardial integrity due to forces generated by fiber cross-fiber shear strains during myofiber contraction. Scarce information on the distribution of $\alpha_{h}$ in the SIT LV is used to set a non-zero initial condition for $\alpha_{h}$ (figure 3.4) in the adaptation model. We performed three SIT simulations in which the longitudinal location of the transition between the normal and inverted transmural distribution of $\alpha_{h}$ is varied. The transition is located halfway between base and apex in simulation MID, more towards the base in simulation $B A S E$, and more towards the apex in simulation APEX. It is expected that variation in this location might explain the inter-individual differences in torsion in SIT. In absence of experimental data, the initial condition for $\alpha_{t}$ was set to zero. For reference purposes, a situs solitus simulation SS was performed in which a normal initial distribution of $\alpha_{h}$ was set [29]. As adaptation proceeded, we analyzed local and global LV function and compared model computed torsion with experimental torsion data. 

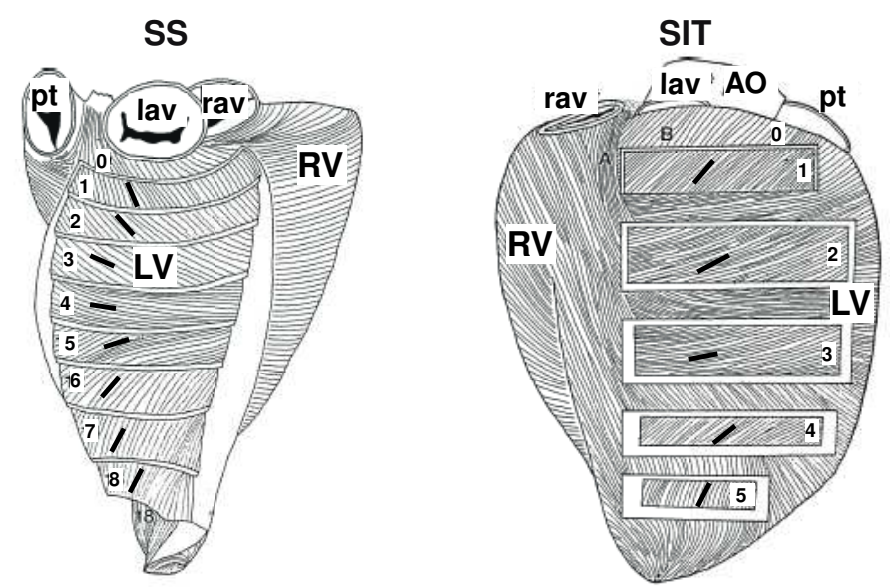

Figure 3.1: Direction of the myofibers in Situs Solitus (left) and Situs Inversus Totalis (right) at several heights between base and apex, and several depths from epicardium to endocardium (indicated by the numbers) [24]. AO: aorta; LAV: left atrioventricular orifice, LV: left ventricle; PT: pulmonary trunk; RAV: right atrioventricular orifice; RV: right ventricle.

\subsection{Methods}

\subsubsection{Ethics statement}

All subjects gave informed consent prior to enrolment in the study, in accordance to the joint ethical committee of Maastricht University and Academic Hospital Maastricht.

\subsubsection{Model of left ventricular mechanics}

Tissue deformations during the cardiac cycle are calculated with a generic finite element (FE) model of LV mechanics. With respect to geometry, material properties and the circulation in which the LV is embedded, this FE model is identical to the model presented in [4]. Therefore, it will only be described in brief.

Geometry In the passive stress-free state, a thick-walled geometry is assumed (figure 3.3A). The endocardial and epicardial surfaces are described by truncated ellipsoids. In this state, wall and cavity volumes equal $140 \mathrm{ml}$ and $40 \mathrm{ml}$, respectively. 


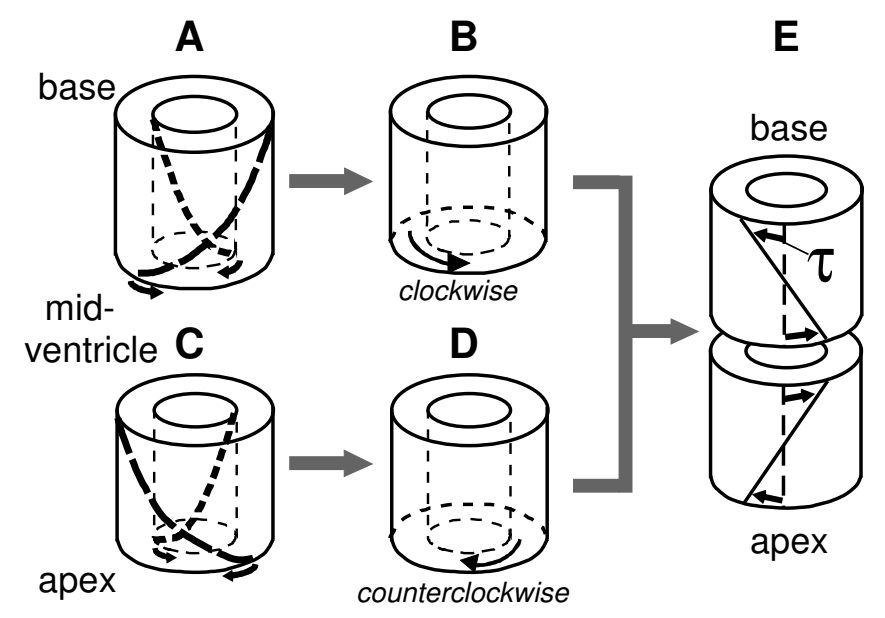

Figure 3.2: Relation between myofiber orientation and torsion. Top: At the base of the Situs Inversus Totalis (SIT) LV, myofibers follow a right-handed helical path at the sub-epicardium (A). Contraction of these myofibers, tends to rotate the midventricle in a clockwise direction with respect to the base, when viewed from the apex. The opposite is true for the sub-endocardium: myofibers follow a left-handed helical path, and contraction of these myofibers tends to rotate the midventricle in a counterclockwise direction with respect to the base (A). In general, a net clockwise rotation is measured at the base in SIT LV [8], indicating that epicardial myofibers dominate endocardial myofibers (B). Bottom: At the apex of the SIT LV, myofibers follow the same pattern as in the Situs Solitus (SS) LV. The dominant myofibers at the sub-epicardium follow a left-handed helical path $(\mathbf{C})$. During contraction, a net counterclockwise rotation is measured in the apical region with respect to the midventricle of the SIT LV or with respect to the base in the SS LV (D). In fact, C and D both represent a whole SS LV. Finally, the torsion angle $\tau$ in SIT is similar to SS at the apex and inverted at the base (E). 
Material properties Myocardial tissue Cauchy stress $\sigma$ is composed of a passive component $\sigma_{p}$ and an active component $\sigma_{a}$ :

$$
\sigma=\sigma_{p}+\sigma_{a} \vec{e}_{f} \vec{e}_{f}
$$

with $\vec{e}_{f}$ the current myofiber direction in the deformed tissue. Passive material behavior is assumed nonlinearly elastic, transversely isotropic, and nearly incompressible. The mathematical description of the strain energy density function can be found in [4] and is based on experiments in dogs [35].

Active stress $\sigma_{a}$ is modeled through a series arrangement of a contractile and a series elastic element. The magnitude of $\sigma_{a}$ depends on time elapsed since activation $t_{a}$, sarcomere length $l_{s}$, and sarcomere shortening velocity $-\partial l_{s} / \partial t[17]$ :

$$
\sigma_{a}=f\left(t_{a}, l_{s},-\frac{\partial l_{s}}{\partial t}\right)
$$

Principles of the active material behavior are based on experiments in dogs [2]. Parameters values for the active material are derived from experiments in rats $[16,18]$. Active stress development is initiated simultaneously at each location in the LV wall with a cycle time of $800 \mathrm{~ms}$.

Governing equations and boundary conditions In the model, the quasistatic equations of conservation of linear momentum are solved:

$$
\vec{\nabla} \cdot \sigma=\overrightarrow{0}
$$

with $\vec{\nabla}$ the spatial gradient operator. At the base, essential boundary conditions are defined to suppress rigid body motion and to represent the mechanical effect of structures left out of the model, e.g., the valvular annulus. Axial displacement is suppressed at the whole basal surface, whereas circumferential displacement is suppressed at the endocardial basal ring only. The epicardial surface is assumed to be traction free while the endocardial surface is uniformly subjected to left ventricular pressure $p_{l v}$. During isovolumic contraction (IC) and relaxation (IR) phases of the cardiac cycle, $p_{l v}$ is determined such that mechanical equilibrium of the myocardial tissue is obtained at a constant end-diastolic or end-systolic LV volume, respectively. During the filling and ejection phase, $p_{l v}$ is computed from the interaction of the LV with the circulation according to a lumped parameter model [4] (figure 3.3A). Parameter values of the circulation model are based on the human hemodynamic system.

Numerical implementation The equilibrium equations (5.4) are solved numerically with a Galerkin type finite element method using 27-noded hexahedral elements with a tri-quadratic interpolation of the displacement field. Because the model is rotationally symmetric, tissue displacements are described 
in a right-handed cylindrical coordinate system $\left\{\vec{e}_{z}, \vec{e}_{r}, \vec{e}_{c}\right\}$ with the axial direction $\vec{e}_{z}$ defined from apex-to-base. This allows description of the LV wall with 1 circumferential element, which reduces computational demand significantly. In total, the LV wall is represented by 60 elements: 6 elements in radial, 1 in circumferential and 10 in longitudinal direction.

\subsubsection{Myofiber orientation}

The myofiber orientation $\vec{e}_{f, 0}$ is prescribed with respect to the local cardiac coordinate system $\left\{\vec{e}_{l, 0}, \vec{e}_{t, 0}, \vec{e}_{c, 0}\right\}$ (figure 3.3B), where the subscript 0 refers to the mechanically unloaded state. The transmural direction $\vec{e}_{t, 0}$ is defined as the outer normal to the cardiac surfaces. The longitudinal direction $\vec{e}_{l, 0}$ is defined perpendicular to $\vec{e}_{t, 0}$ from apex to base. To obtain a right-handed coordinate system, the circumferential direction $\vec{e}_{c, 0}$ is defined in clockwise direction when viewing the LV in apex-to-base direction. Myofiber orientations are described by two angles. The helix angle $\alpha_{h, 0}$ is defined as the angle between $\vec{e}_{c, 0}$ and the projection of $\vec{e}_{f, 0}$ on the circumferential-longitudinal plane $\left(\vec{e}_{c, 0}, \vec{e}_{l, 0}\right)$. The transverse angle $\alpha_{t, 0}$ is defined as the angle between $\vec{e}_{c, 0}$ and the projection of $\vec{e}_{f, 0}$ on the circumferential-transmural plane $\left(\vec{e}_{c, 0}, \vec{e}_{t, 0}\right)$.

\subsubsection{Myofiber reorientation}

We simulated myofiber reorientation with the model by Kroon et al. [19]. In this model, it was assumed that structural changes of myofiber orientation occur as a response to local loss of myocardial integrity due to forces generated by fiber cross-fiber shear strains during myofiber contraction. These shear forces are assumed to damage connections between extra-cellular matrix (ECM) and myofibers. New connections are formed continuously during both the diastolic and systolic phase of the cardiac cycle. When a connection is made, the actual orientation field tends to be fixed within the tissue. This conceptual model was translated into a mathematical model in which the myofiber orientation in the unloaded state $\vec{e}_{f, 0}$ will evolve towards the actual myofiber orientation $\vec{e}_{f}$ corrected for rigid body rotation. In a previous study, we have shown that this mechanism leads to a realistic myofiber orientation pattern in the SS LV [19]. In particular, a non-zero $\alpha_{t}$ developed, that caused improved correspondence between model predicted and experimentally determined patterns of LV circumferential-radial shear strain and torsion [21].

\subsubsection{Simulations performed}

One SS simulation and three SIT simulations were performed. In all simulations, the first 10 consecutive cardiac cycles were used to reach a hemody- 
namic steady state and myofiber reorientation was not included. In subsequent cycles myofiber reorientation was simulated throughout the whole LV.

Initial myofiber orientation in SS At the start of the adaptation process, the transmural distribution of $\alpha_{h, 0}$ in SS $\left(\alpha_{h, 0}^{S S}\right)$ is described using the parameterized distribution in [4]. It varies nonlinearly with the transmural position from endocardium to epicardium (figure 3.8, dashed lines in left graph). This spatial distribution is a function of normalized coordinates ( $u, v$, figure 3.4B). The normalized longitudinal coordinate $(u)$ varies linearly with the geodesic distance from the equatorial plane. It changes from $u=+0.5$ in the basal plane, through $u=0$ at the equator to $u=-1$ at the apex. The normalized transmural coordinate $(v)$ varies linearly with the distance in the ventricular wall from $v=-1$ at the endocardial surface to $v=1$ at the epicardial surface. The initial condition for transmural distribution of $\alpha_{t, 0}$ is set to zero.

In both SS and SIT the parameterized description of fiber orientation is abandoned during adaption, and myofiber orientation is adapted per node.

Initial myofiber orientation in SIT According to anatomical data of SIT LVs, the helix angle $\alpha_{h, 0}$ must change from a normal transmural course at the apex to an inverted transmural course at the base [24]. Inverted $\alpha_{h, 0}\left(\alpha_{h, 0}^{i n v}\right)$ is defined as

$$
\alpha_{h, 0}^{i n v}=-\alpha_{h, 0}^{S S}
$$

The initial transition from an SS to an inverted pattern is characterized by three parameters: the location at midwall between apex and base $u_{\text {mid }}$, the slope of the transition between the endo- and epicardium $\gamma$, and the height of the transition zone $h$ (figure 3.4B). In the LV region below the transition zone, the transmural course of $\alpha_{h, 0}$ is as in the SS LV $\left(\alpha_{h, 0}^{S S}\right)$. In the region above the transition zone, the transmural course of $\alpha_{h, 0}$ follows an inverted pattern $\left(\alpha_{h, 0}^{i n v}\right)$. Across the transition zone, $\alpha_{h, 0}$ changes linearly from $\alpha_{h, 0}^{S S}$ to $\alpha_{h, 0}^{i n v}$ (figure $3.4 \mathrm{~B}$ and $\mathrm{C}$ ). As in simulation $S S$, the initial transmural distribution of $\alpha_{t, 0}$ is set to zero.

In this study, the location of the transition zone $u_{\text {mid }}$ was subject of variation, whereas $\gamma$ and $h$ remained unchanged. The transition is located at $u_{\text {mid }}=-0.25$ in simulation MID, more towards the base $\left(u_{\text {mid }}=0\right)$ in simulation BASE, and more towards the apex $\left(u_{\text {mid }}=-0.5\right)$ in simulation APEX. In the additional simulations (results presented in figure 3.11), $\gamma$ was subject of variation while $u_{\text {mid }}$ and $h$ remained unchanged.

\subsubsection{Postprocessing}

Quantification of LV function Local function was quantified by changes in mean and standard deviation (SD) of four parameters quantifying mechanical 

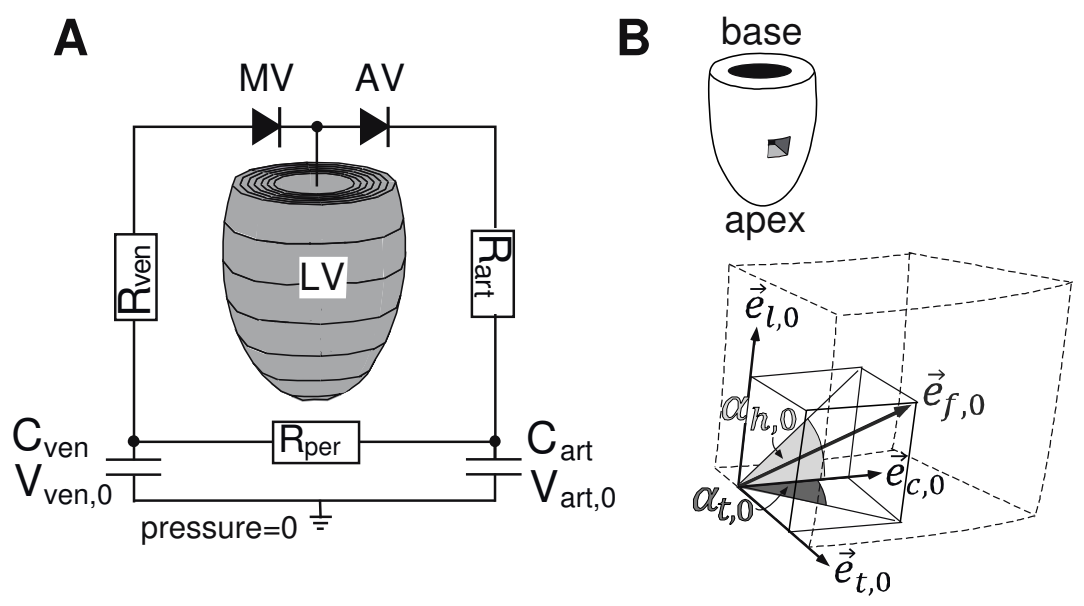

Figure 3.3: Computational model of LV mechanics. A: The ellipsoidally shaped finite element (FE) mesh of the left ventricle (LV) consists of 60 elements and is incorporated in a lumped parameter model of the circulation. $\mathrm{AV}$, aortic valve; $C_{\text {art }}$, arterial compliance; $C_{v e n}$, venous compliance; $\mathrm{MV}$, mitral valve; $R_{\text {art }}$, arterial resistance; $R_{\text {per }}$, peripheral resistance; $R_{\text {ven }}$, venous resistance; $V_{\text {art }, 0}$, zero-pressure arterial volume; $V_{\text {ven,0, }}$, zero-pressure venous volume. B: Description of myofiber orientation vector in the unloaded state $\vec{e}_{f, 0}$ by helix angle $\alpha_{h, 0}$ and transverse angle $\alpha_{t, 0}$ using a local cardiac coordinate system $\left\{\vec{e}_{l, 0}, \vec{e}_{t, 0}, \vec{e}_{c, 0}\right\}$.

tissue load: stroke work density $w_{f}$, and natural myofiber strain during isovolumic contraction $\epsilon_{f, i c}$, during ejection $\epsilon_{f, e j}$, and during isovolumic relaxation $\epsilon_{f, i r}$. The $w_{f}$ at each point is defined as the area enclosed by the myofiber Cauchy stress-natural strain loop:

$$
w_{f}=\oint \sigma_{f} d \epsilon_{f} ; \quad \epsilon_{f}=\ln \lambda_{f}
$$

Global cardiac function is quantified by maximum LV pressure $p_{l v, \text { max }}$ and stroke volume $S V$.

Comparison with experimental data Left ventricular torsion $\tau$ as computed with the model was compared with torsion determined from magnetic resonance tagging (MRT) experiments. The protocol for the MRT measurements and the definition of $\tau$ have been described previously in [8]. Essentially, $\tau$ quantifies the base-to-apex gradient of rotation about the LV long axis (see figure 3.2). In the model, $\tau$ was determined at the levels of the MR slices by interpolation from adjacent nodal points in the FE mesh. In agreement with the experimental procedure, $\tau$ was computed with respect to begin-ejection and averaged in radial direction. 
A

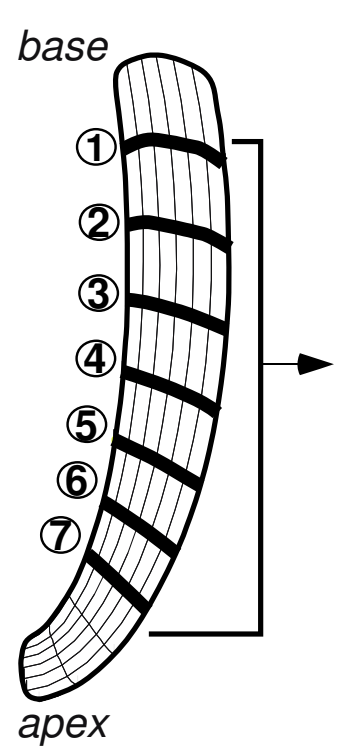

B

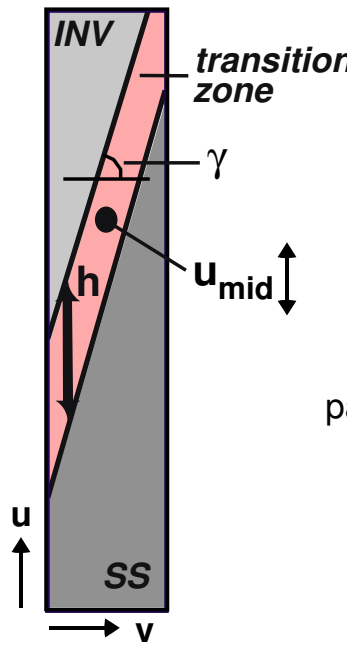

C

Helix angle

[deg]

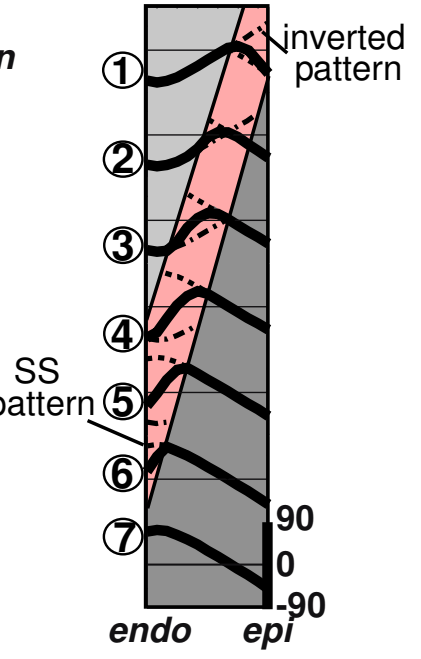

Figure 3.4: Initial myofiber orientation in SIT simulation MID. A: Long-axis crosssection of LV mesh. B: Mapping of the cross-section in A on a rectangular domain used to describe the spatial distribution of myofiber orientations. The transition from a normal (SS) pattern at the apex to an inverted (INV) pattern at the base is described by parameters $u_{\text {mid }}$ (location of transition at midwall), $\gamma$ (the slope of the transition between the endo- and epicardium), and $h$ (the height of the transition zone). $u_{\text {mid }}$ is subject of variation in between the SIT simulations BASE, MID and APEX. C: Initial (before reorientation) transmural course of helix angle $\alpha_{h, 0}$ in simulation MID (solid) at the 7 levels indicated in A. In the transition zone, the courses of the SS (- -) and inverted pattern (- .) are presented. The transmural distribution of the transverse angle $\alpha_{t, 0}$ was set to zero. 


\subsection{Results}

In all simulations, local and global LV function increased significantly during the adaptation process as indicated by the increase in 1) myofiber shortening (decrease of myofiber strain) during ejection $\epsilon_{e j}$, 2) stroke work density $w_{f}$ (area enclosed by myofiber Cauchy stress-natural myofiber strain loop), 3) maximum left ventricular pressure $p_{l v, \max }$, and 4) stroke volume $S V$. In addition, fiber strains during the isovolumic contraction (IC) and relaxation (IR) phases, $\epsilon_{i c}$ and $\epsilon_{i r}$, decreased significantly as a result of minimizing fiber cross-fiber shear. As an example, evolution of local and global function in simulation MID is shown in figure 3.5. Parameter values all reached a steady state value after about 15 adaptation cycles. In the steady state, standard deviations (SD) of the local function parameters are significantly decreased, which indicates increase in homogeneity. For example, the SD of $w_{f}$ decreased with $\sim 30 \%$.

After 15 adaptation cycles, function parameter values are not significantly different between the SIT simulations. Neither are the values in the SIT simulations significantly different in comparison to the SS simulation (figure 3.6). In simulation $S S, \epsilon_{e j}$ exhibits less heterogeneity when compared to the SIT simulations.

Local myocardial function in simulation MID is shown in more detail in figure 3.7. Myofiber Cauchy stress-natural strain loops are shown before (dashed line) and after 15 adaptation cycles (solid line) in several nodes across the LV wall. After reorientation, the loops become more homogeneous, as was also indicated by the decrease in SD of myofiber strains and stroke work density (figure 3.5). Although homogeneity increased significantly, locations in or near the transition zone in the SIT LV still show deviating local myocardial function after reorientation. This results in, for example, a larger SD in $\epsilon_{e j}$ when compared to simulation SS (figure 3.6).

Figure 3.8 shows transmural distributions of the helix angle $\alpha_{h, 0}$ and transverse angle $\alpha_{t, 0}$ in the mechanically unloaded state, indicated by subscript 0 (see figure 3.3B). Results are shown before (dashed line) and after 15 adaptation cycles (solid line) at 7 levels between apex and base. In all simulations, relatively small changes are observed between initial and final distributions of $\alpha_{h, 0}$. Though $\alpha_{h, 0}$ changed, especially in the transition zone, transmural patterns stayed present.

Larger changes are observed between initial and final distributions of $\alpha_{t, 0}$. In simulation $S S, \alpha_{t, 0}$ develops a characteristic pattern from positive basal values to negative apical values at midwall. In the SIT simulations, the pattern is more complex. In simulation MID, $\alpha_{t, 0}$ at the basal level varies from negative values at the endocardium to positive values at the epicardium. Going from base to apex, the region of positive $\alpha_{t, 0}$ shifts towards the endocardium and 
a region of negative $\alpha_{t, 0}$ develops near the epicardium. In simulations $B A S E$ and APEX the pattern is similar, except for a shift towards base and apex, respectively.

In figure 3.9, myofiber angles $\alpha_{h, 0}$ and $\alpha_{t, 0}$ are shown on a long axis crosssection of the LV mesh. Since fiber angles in the SIT simulations are generally the same, results of SIT simulation MID are presented next to the results of SS to visualize the difference in distribution pattern between SIT and SS. During adaptation, $\alpha_{h, 0}$ changes more in SIT than in SS, especially in the transition zone. In SS, the transmural course of helix angle $\alpha_{h}$ is qualitatively the same from apex-to-base, whereas in SIT the transmural course of $\alpha_{h}$ changes from apex-to-base. In SS, the maximum amplitude of $\alpha_{t, 0}$ is located near the endocardium and changes from negative at the apex to positive at the base. In SIT, maximum $\alpha_{t, 0}$ is located near the endocardium at the apex, but shifts towards the epicardium near the base. In addition, largest values of $\alpha_{t, 0}$ appear in the transition zone. In this respect, all SIT simulations resulted in similar structures. Though, in simulation $B A S E$, the area with highest amplitudes of $\alpha_{t, 0}$ is located more towards the base, and in simulation APEX more towards the apex. The translation of fiber angle distributions into a 3-D structure is presented in figure 3.9C. From 10 different points between endo- and epicardium but at the same level between apex and base, fiber paths were followed resulting in the partially filled LV as shown in the figure.

Figure 3.10 shows the results of torsion patterns in both model (left) and experiment (right). After reorientation, torsion patterns have changed significantly, especially in the SIT simulations. Torsion amplitudes after reorientation $(\sim 0.10 \mathrm{rad})$ are significantly lower than those before reorientation $(\sim 0.40$ $\mathrm{rad})$ and more in agreement with experimentally observed amplitudes $(\sim 0.15$ $\mathrm{rad})$. After reorientation, the torsion patterns, which are negative in the SS $\mathrm{LV}$, become less negative in simulation BASE and may even invert in simulation $A P E X$ during ejection. Thus, the torsion patterns shift along with the transition zone. For each of the torsion patterns in the SIT simulations, a corresponding pattern could be found in the experimental data set of 8 SIT subjects in [8]. In simulation SS, torsion is homogeneous between the sections, which is observed in all 9 SS subjects in [8] as well.

\subsection{Discussion}

In this study, different SIT LV structures were estimated using a finite element (FE) model of LV mechanics [4] including shear-induced myofiber reorientation [19]. In comparison to our previous study [20], geometry is more realistic and fibers are allowed to crossover between endo- and epicardium. Fibers reorient as a response to shear instead of shortening during ejection, and no 
Local function
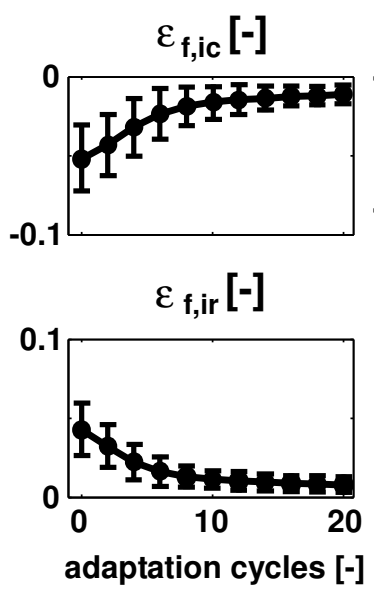

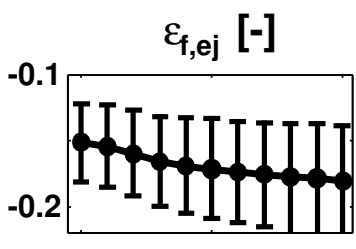

$\mathbf{w}_{\mathrm{f}}[\mathrm{mJ} / \mathrm{ml}]$

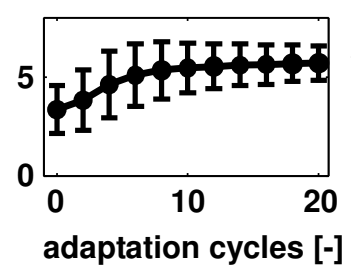

Global function

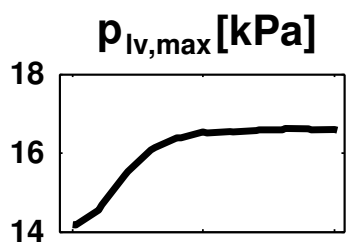

SV [ml]

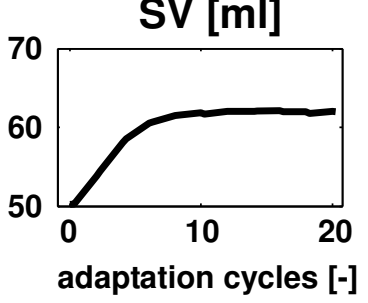

Figure 3.5: Evolution of local (left) and global (right) LV function in SIT simulation MID during the reorientation process. Local function is presented by means and standard deviations (SD) of variables natural myofiber strain during isovolumic contraction $\epsilon_{i c}$, during ejection $\epsilon_{e j}$, during isovolumic relaxation $\epsilon_{i r}$, and stroke work density $w_{f}$. The values were calculated from the grey area indicated in the long-axis cross-section of the LV mesh (mid). Global function is presented by maximum LV pressure $p_{l v, \text { max }}$ and stroke volume $S V$.

constraints are prescribed for fiber orientation at apex or base, allowing the structure to develop without restrictions. Although model set ups are different, this study also showed that local (myofiber) and global (pump) function in the SIT LV is similar to that in the SS LV. In contrast to the previous study, we now showed the possibility of multiple SIT LV structures and the importance of the transverse angle. The final distributions of the helix angle and transverse angle could be considered as the first detailed suggestion for fiber orientations in SIT.

In figure 3.9 it was shown that although the final SIT LV structure is essentially different from the final SS LV structure, it is a continuous structure. Fibers followed a path through the whole ventricular wall, as in the SS LV. Although no experimental data is available to confirm the model predicted structures, the similarities in model computed and experimental torsion indicate that the estimated structures might be realistic.

As a consequence of myofiber reorientation, local and global LV function increased significantly in all simulations. This suggests that, as in the SS LV, mechanical work could indeed be distributed homogeneously in the SIT LV too. Moreover, the location of the transition from a normal myofiber orientation pattern at the apex to an inverted pattern at the base had no influence on 
Local
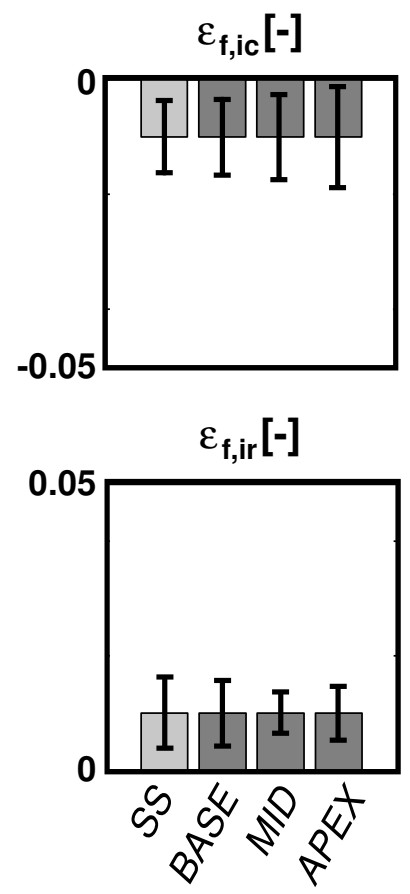
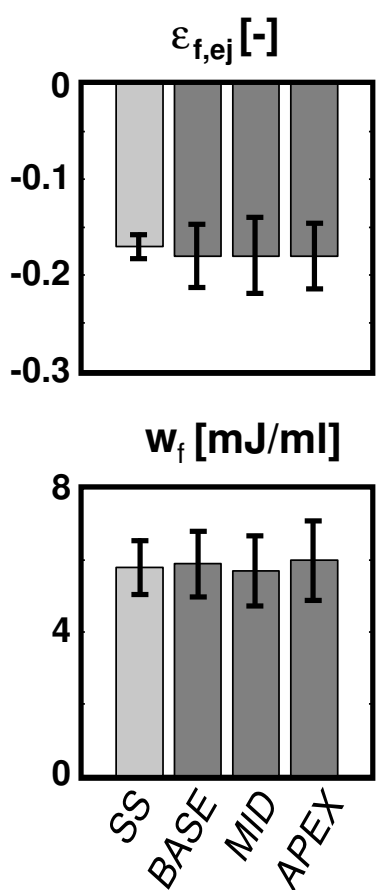

Global

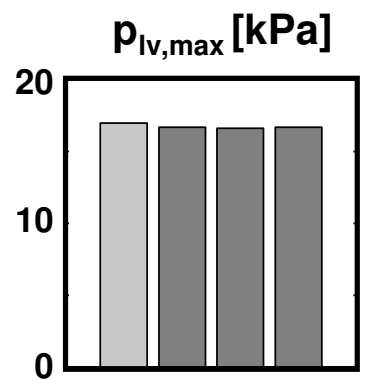

SV [ml]

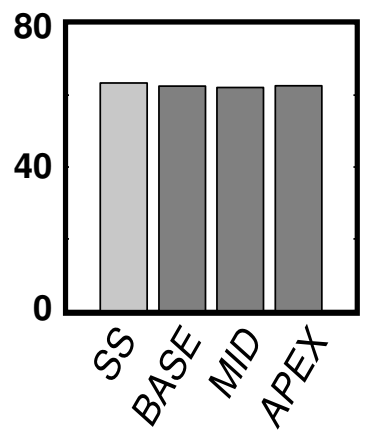

Figure 3.6: Final LV function after 15 adaptation cycles in the SS simulation and the three SIT simulations (BASE, MID, APEX). Mean values and standard deviation (SD) of local function parameters are presented left, values of global parameters right. Differences between simulations are not significant.

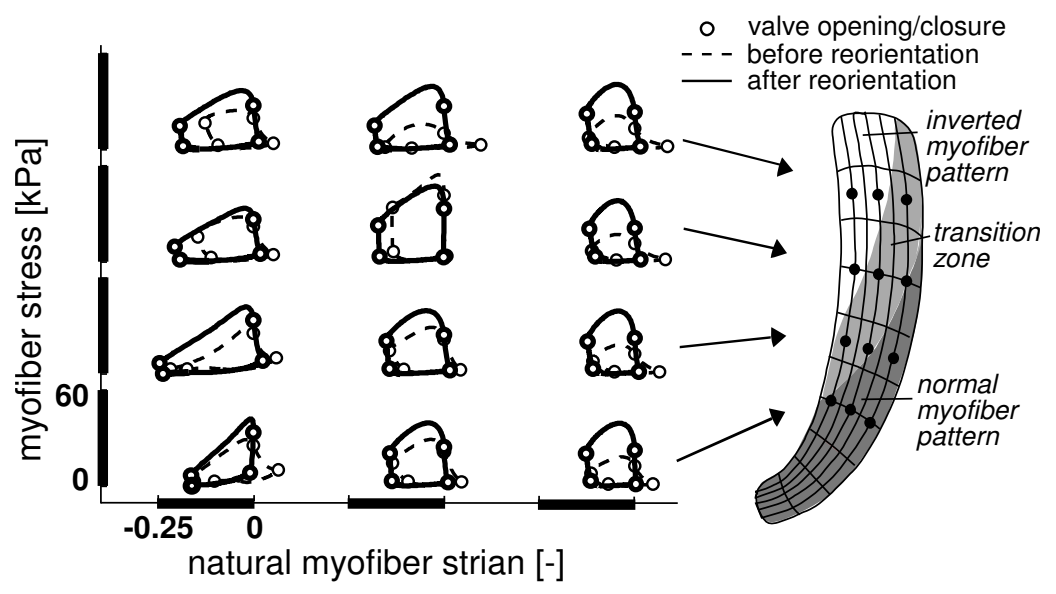

Figure 3.7: Change in local mechanics as a consequence of myofiber reorientation in SIT simulation MID. Myofiber Cauchy stress-natural strain loops are analyzed in the nodes indicated in the LV mesh (right) before (- -) and after (-) reorientation. The location of the transition zone before reorientation is also indicated in the LV mesh. 
SS

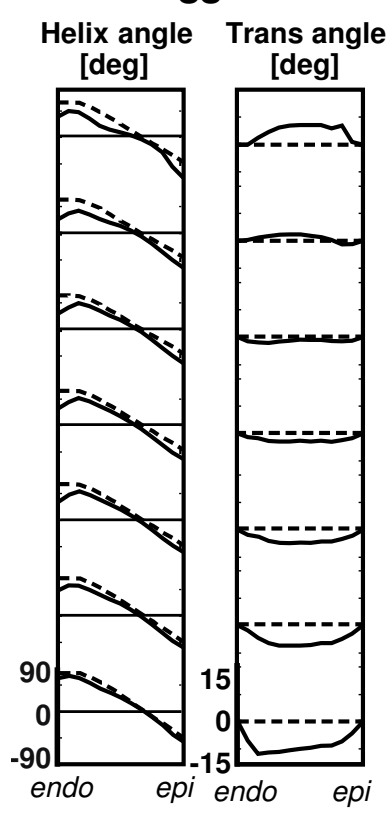

BASE

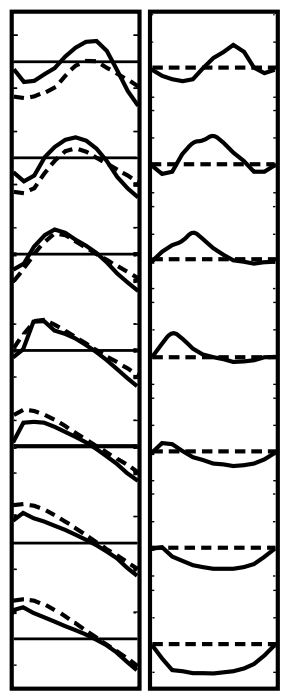

MID

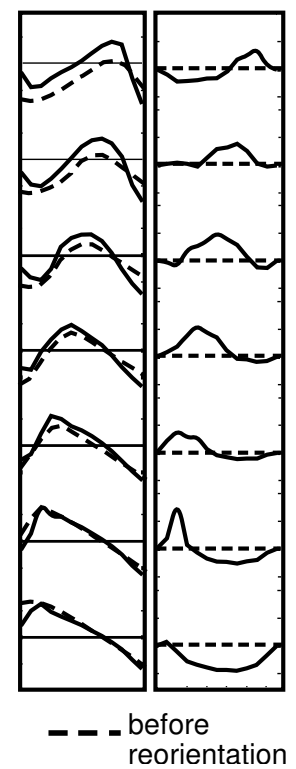

APEX

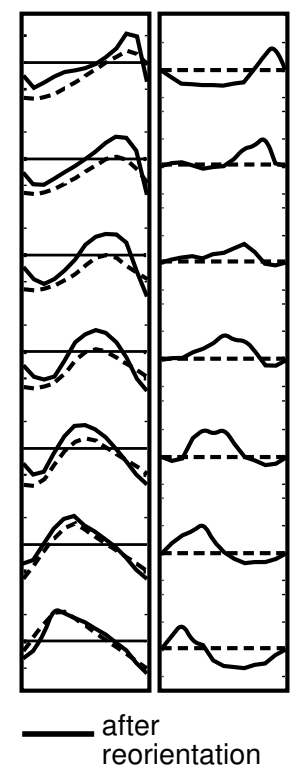

Figure 3.8: Transmural course in myofiber angles before (- -) and after (-) myofiber reorientation. Results of simulation NORM (left), and SIT simulations BASE, MID and APEX (right) are shown. Analysis is done at 7 different levels in the LV wall (see figure $3.4 \mathrm{~A})$. 
A
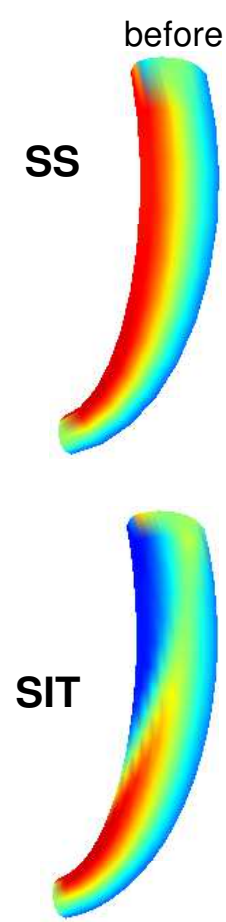

B

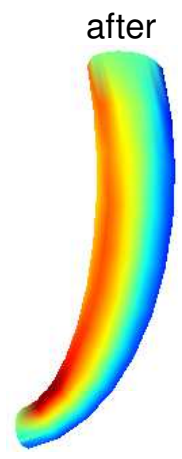

Helix angle

[deg]
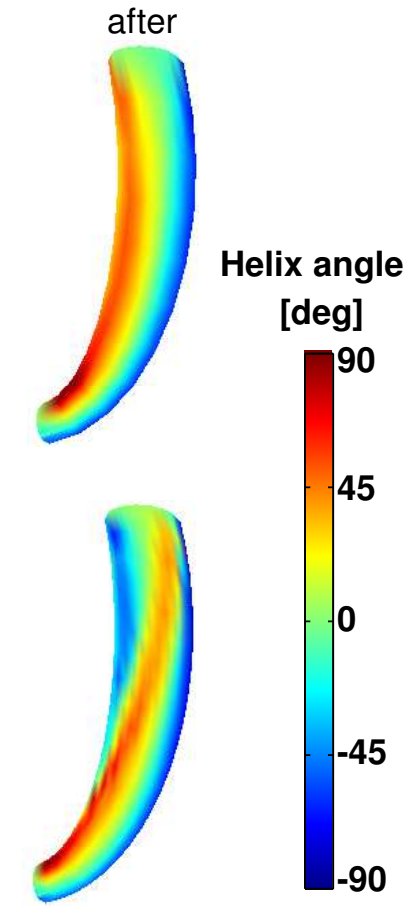

before

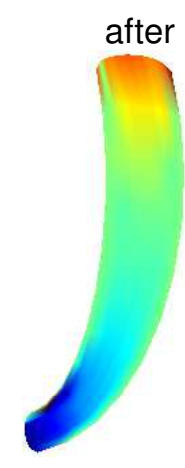

after
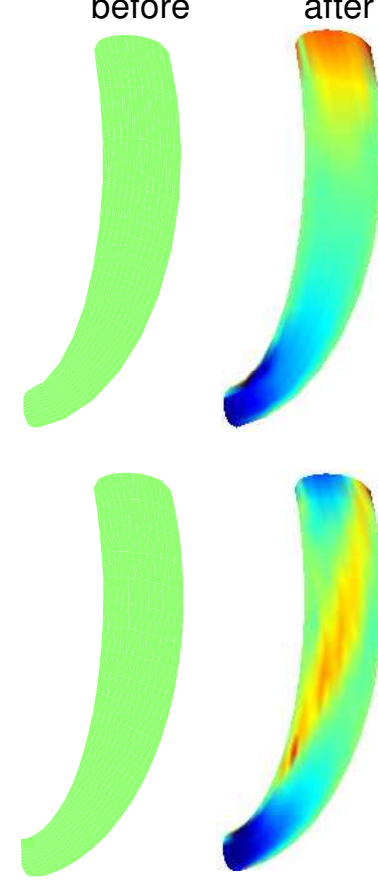

Trans angle

[deg]

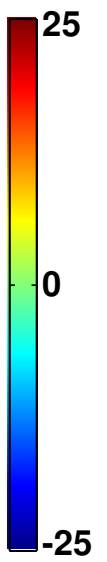

C
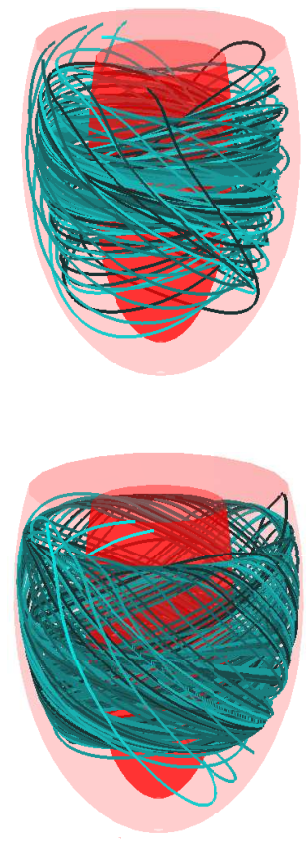

Figure 3.9: Structural results of simulation SS (top row) and SIT simulation MID (bottom row). A: Helix angle $\alpha_{h, 0}$ distribution on a long axis cross-section of the LV mesh before (left) and after (right) reorientation. B: Transverse angle $\alpha_{t, 0}$ distribution before (left) and after (right) reorientation. C: 3-D visualization of fiber paths through the LV mesh after reorientation. Ten paths are shown which started at different locations between endo- and epicardium. The color of the path refers to the starting point. 


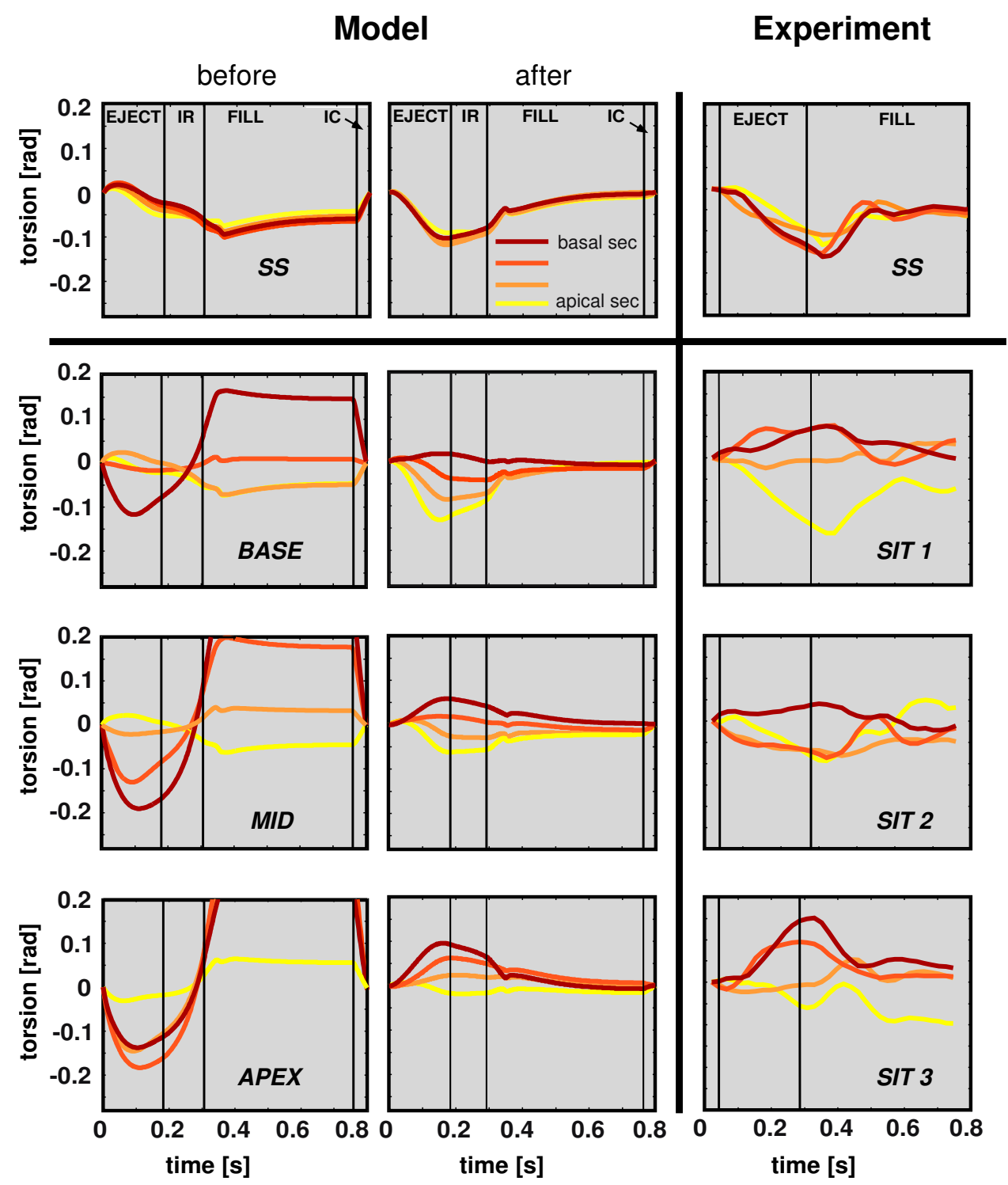

Figure 3.10: Torsion [rad] during a cardiac cycle. Results are presented from simulation SS (upper left), an SS subject (upper right), 3 SIT simulations (bottom left), and 3 SIT subjects (bottom right). Model results are shown before and after reorientation. Torsion was determined in four sections between apex and base. EJECT=ejection phase; $I R=$ isovolumic relaxation phase; $F I L L=$ filling phase; $I C=$ isovolumic contraction phase. 


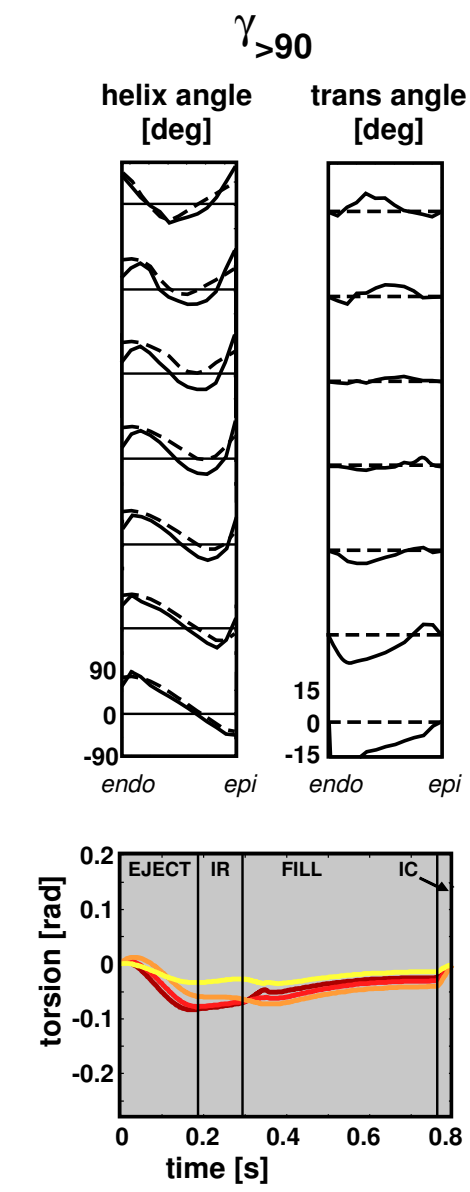

\section{$\gamma_{90}$}

$\gamma_{0}$
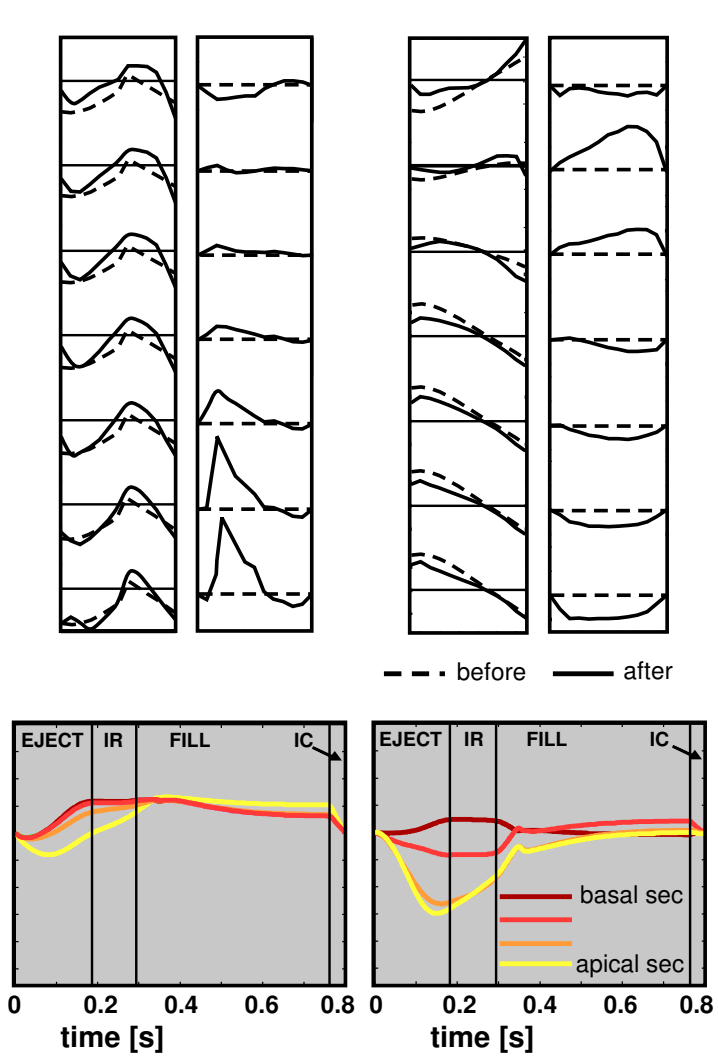

Figure 3.11: Results of additional simulations. The additional simulations were performed with a variation on simulation MID (with $\gamma_{\text {mid }}$, see figure 3.4 for definition of $\gamma): \gamma_{>90}\left(\gamma_{>90}=180-\gamma_{\text {mid }}\right), \gamma_{90}$, and $\gamma_{0}$, where the subscript 90 and 0 refer to a $\gamma$ of $90^{\circ}$ and $0^{\circ}$, respectively. Top: Transmural distribution of $\alpha_{h, 0}$ and $\alpha_{t, 0}$ before (- -) and after 15 adaptation cycles (-) at 7 levels between apex and base. Major pattern of $\alpha_{h, 0}$ remained closed to the initial distribution, while a non-zero distribution for $\alpha_{t, 0}$ developed. Bottom: Torsion patterns [rad] after 15 adaptation cycles. Amplitudes of torsion have decreased significantly after reorientation as compared to amplitudes before reorientation. 
the local and global SIT LV function. Finally, SIT LV function was comparable to SS LV function, which is in agreement with the finding that SIT individuals display no cardiac complaints [8].

The choice of simulations with fixed $\gamma$ and $h$, and a variation in $u_{\text {mid }}$ was based upon the scarce available data on myofiber orientation $[3,24]$ and deduced from experimental findings on torsion [8]. As far as we know, other SIT structures, for example characterized by a substantial variation in $\gamma$, are not reported in literature. Yet, to investigate the space of feasible solutions, we performed additional simulations. These new simulations are a variation on simulation MID (with $\gamma_{\text {mid }}$ ): $\gamma_{>90}$ (with $\gamma_{>90}=180^{\circ}-\gamma_{\text {mid }}$ ), $\gamma_{90}$, and $\gamma_{0}$, where the subscript 90 and 0 refer to a $\gamma$ of $90^{\circ}$ and $0^{\circ}$, respectively. In all additional simulations, both local and global LV function developed according to the patterns shown in figure 3.5. In addition, LV function after 15 adaptation cycles was not statistically different from that shown in figure 3.6. Fiber orientation also developed similarly to the results shown in figure 3.9: after adaptation, the distribution of $\alpha_{h}$ was still close to the initial distribution, while $\alpha_{t}$ developed a non-zero distribution (see figure 3.11). Torsion amplitude decreased significantly upon adaptation. Consequently, our model predicts the existence of many fiber architectures, characterized by a case specific match of the distributions of helix and transverse angles.

This finding seems to contradict the finding of our previous study in the SS LV, where we concluded that the effect of the initial condition of the helix angle disappeared upon adaptation [28]. Apparently, in the latter study the initial conditions for $\alpha_{h}$ were close enough for the remodeling process to end up in the same solution. In our SIT simulations BASE, MID and APEX, the differences in initial conditions persist after remodeling, although they are limited to a base-to-apex shift of the transition zone only. Out of the many possible solutions predicted by our model, only the SIT structures and the SS structure are found in practice. This suggests that additional physiological mechanisms exist, that regulate myofiber orientation.

Comparison with experimental data In figure 3.10 it is shown that agreement between model computed and experimentally determined torsion is significantly better after reorientation. The agreement suggests that the estimated structures could indeed be realistic. Thus, an abnormal torsion pattern could coincide with normal LV function [10]. The inter-individual differences in torsional deformation could originate from a different location of the transition in LV structure from normal at the apex to inverted at the base.

Because of relaxation of spins in the magnetized tissue, the strength of the MR signal decreases over time. This makes tracking of the tags more difficult at the end of the filling phase. The decrease in reliability of the estimation 
of torsion towards the end of the cardiac cycle is evident from the non-zero values of torsion amplitude: considering the cyclic deformation of the myocardium, these values are expected to return to zero.

The maximum amplitude of torsion occurred earlier in the model than in the experiment. This observation indicates that the timing of increase and decrease of active stress development in the model is not entirely realistic. However, the difference in timing had no influence on the increase in homogeneity in function nor on the gradient in torsion amplitude, which was developed in the SIT simulations after reorientation.

Study assumptions and limitations The results of the shear-induced adaptation may have been influenced by the absence of sheets in the constitutive model of the tissue. Sheets are predominantly oriented in transmural direction, facilitating thickening of the wall $[22,27]$. Their effect on normal and shear stiffness of the tissue has been demonstrated in experiments [9] and quantified in constitutive models [31]. Similar to the hypothesis on myofiber reorientation that we used to estimate myofiber orientations, the orientation of these sheets has been linked to shear as well [1]. As such, extension of the adaptation model by including sheets and reorientation thereof could be considered as a next step.

As mentioned before, other adaptation mechanisms are likely to be active as well. Clinically, one of the most evident examples of adaptation is the change in LV wall mass and cavity volume in response to pressure and volume overload, respectively. In addition, in reality the externally unloaded LV exhibits a transmural gradient in sarcomere length with epicardial sarcomeres being longer than endocardial ones [30]. This might be a result of mechanically induced adaptation as well. Extension of the model with these adaptive mechanisms should be considered.

In this study, torsional deformation was used to compare results of model and experiment. We also compared model predictions of the deformation mode circumferential-radial shear to experimental data. Similar to our previous study for SS [21], circumferential-radial shear decreased substantially upon fiber reorientation, but final patterns did not match experimental findings. The discrepancy is mainly explained by the large sensitivity of this shear component to the setting of $\alpha_{t}$ [34]. In addition, the discrepancy suggests that our model of shear-induced remodeling of fiber orientation must be complemented by other remodeling laws.

Our cardiac mechanics model has several limitations. For example, the onset of contraction was assumed to be homogeneous, despite the fact that there is a delay in electrical activation of about $40 \mathrm{~ms}$. This assumption is motivated by the observation that, at least in the normal healthy heart, a homogeneous 
onset of contraction yields more realistic strains than assuming the timing of the onset of contraction to follow the electrical activation [17]. LV shape, the major determinants of which are the ratio of cavity to wall volume and eccentricity, was based on data from dog hearts [26, 32]. LV size was set to $140 \mathrm{ml}$, the average volume of the dog hearts used to validate the original model [5]. This volume is representative for a small human heart as well, as indicated by the cardiac output of about $4.9 \mathrm{l} / \mathrm{min}$ in our simulations. Since tissue mechanics does not depend on absolute size and the influence of shape is minor [12], we consider our description of LV geometry adequate for this study.

Geometry and structure of the LV were assumed rotationally symmetric, while interaction of the LV with the right ventricle (RV) was not taken into account. Myofiber orientations show differences between septum and LV free wall [13] that could originate from the mechanical interaction of LV and RV. If experimental data of myofiber orientations in the SIT LV can be obtained, they should be measured in the free wall, since the effect of interaction will be least for this region. Our predictions on $\alpha_{t}$ suggest that these experiments might focus on the finding that the region of maximum positive $\alpha_{t}$ shifts from the epicardium to the endocardium, when traveling from base to apex.

\subsection{Conclusion}

In this study, we have found that local and global LV function in SIT and SS were similar, despite essential differences in myocardial structure. Using the same processes of shear-induced myofiber reorientation, both SS and SIT LV structures were estimated by this adaptation mechanism and the structures were continuous. The space of feasible solutions predicted by the model turned out to be larger than the experimentally found variation in structures. This suggests that additional physiological mechanisms exist that regulate myofiber orientation. Large agreement in torsion data between model and experiment suggests that measured interindividual differences in torsion pattern could originate from different locations of the transition from a normal myofiber orientation pattern at the apex to an inverted pattern at the base. 


\section{References}

1. Arts T, Costa KD, Covell JW, and McCulloch AD (2001). "Relating myocardial laminar architecture to shear strain and muscle fiber orientation." Am J Physiol Heart Circ Physiol 280.5, $\mathrm{H} 2222-\mathrm{H} 2229$.

2. Arts T, Veenstra PC, and Reneman RS (1982). "Epicardial deformation and left ventricular wall mechanisms during ejection in the dog." Am J Physiol 243.3, H379-H390.

3. Asami I and Koizumi K (1989). "The vortex cordis is never reversely directed, even in situs inversus and L-loop anomaly". Kaibogaku Zasshi 64, pp. 36-45.

4. Bovendeerd PHM, Kroon W, and Delhaas T (2009). "Determinants of left ventricular shear strain". Am J Physiol - Heart Circ Physiol 297.3, H1058-H1068.

5. Bovendeerd P, Arts T, Delhaas T, Huyghe J, Van Campen D, and Reneman R (1996). "Regional wall mechanics in the ischemic left ventricle: numerical modeling and dog experiments." Am J Physiol 270.1 Pt 2, H398-H410.

6. Bovendeerd P, Arts T, Huyghe J, Campen D van, and Reneman R (1992). “Dependency of local left ventricular wall mechanics on myocardial myofiber orientation: a model study". $J$ Biomech 25, pp. 1129-1135.

7. Delhaas T, Kroon W, Bovendeerd P, and Arts T (2008a). "Left ventricular apical torsion and architecture are not inverted in situs inversus totalis". Prog Biophys Mol Biol 97, pp. 513519 .

8. Delhaas T, Kroon W, Decaluwe W, Rubbens M, Bovendeerd P, and Arts T (2008b). "Structure and torsion of the normal and situs inversus totalis cardiac left ventricle; Part I: experimental data in humans". Am J Physiol - Heart Circ Physiol 295, H197-H201.

9. Dokos S, Smaill BH, Young AA, and LeGrice IJ (2002). "Shear properties of passive ventricular myocardium". Am J Physiol 283, H2650-H2659.

10. Frank LH, Yu Q, Francis R, Tian X, Samtani R, Sahn DJ, Leatherbury L, and Lo CW (2010). "Ventricular rotation is independent of cardiac looping: A study with Situs Inversus Totalis using speckle-tracking echocardiography". Journal of the American Society of Echocardiography 23, pp. 315-323.

11. Geerts-Ossevoort L, Bovendeerd P, Nicolay K, and Arts T (2002). “Characterization of the normal cardiac myofiber field in goat measured with MR-diffusion tensor imaging". Am J Physiol - Heart Circ Physiol 283.1, H139-H145.

12. Geerts-Ossevoort L, Kerckhoffs R, Bovendeerd P, and Arts T (2003). “Towards Patient Specific Models of Cardiac Mechanics: A Sensitivity Study". In: Functional Imaging and Modeling of the Heart. Ed. by I Magnin, J Montagnat, P Clarysse, J Nenonen, and T Katila. Vol. 2674. Lecture Notes in Computer Science. Springer Berlin Heidelberg, pp. 81-90.

13. Gilbert S, Benson A, Li P, and Van Holden A (2007). "Regional localisation of left ventricular sheet structure: integration with current models of cardiac fibre, sheet and band structure". Eur J Cardiothorac Surg 32, pp. 231-249.

14. Greenbaum R, Ho S, Gibson D, Becker A, and Anderson R (1981). "Left ventricular fibre architecture in man". Br Heart J 45, pp. 248-263. 
15. Grimm A, Katele K, and Lin H (1976). "Fiber bundle direction in the mammalian heart. An extension of the 'nested shells' model." Basic Res. Cardiol. 71, pp. 381-388.

16. Janssen PM and Hunter WC (1995). "Force, not sarcomere length, correlates with prolongation of isosarcometric contraction." Am J Physiol 269.2 Pt 2, H676-H685.

17. Kerckhoffs RCP, Bovendeerd PHM, Kotte JCS, Prinzen FW, Smits K, and Arts T (2003).

"Homogeneity of cardiac contraction despite physiological asynchrony of depolarization: a model study." Ann Biomed Eng 31.5, pp. 536-547.

18. Keurs HE ter, Bucx JJ, Tombe PP de, Backx P, and Iwazumi T (1988). "The effects of sarcomere length and $\mathrm{Ca}++$ on force and velocity of shortening in cardiac muscle." Adv Exp Med Biol 226, pp. 581-593.

19. Kroon W, Delhaas T, Arts T, and Bovendeerd P (2009a). “Computational analysis of the myocardial structure: Adaptation of myofiber orientations through deformation in three dimensions". Med Imag Anal 13, pp. 346-353.

20. Kroon W, Delhaas T, Bovendeerd P, and Arts T (2008). "Structure and torsion in the normal and situs inversus totalis cardiac left ventricle; Part II: Modeling cardiac adaptation to mechanical load". Am J Physiol - Heart Circ Physiol 295, H202-H210.

21. Kroon W, Delhaas T, Bovendeerd P, and Arts T (2009b). "Adaptive Reorientation of Cardiac Myofibers: Comparison of Left Ventricular Shear in Model and Experiment". In: Functional Imaging and Modeling of the Heart. Ed. by N Ayache, H Delingette, and M Sermesant. Vol. 5528. Lecture Notes in Computer Science. Springer Berlin Heidelberg, pp. 58-67.

22. LeGrice IJ, Takayama Y, and Covell JW (1995). “Transverse shear along myocardial cleavage planes provides a mechanism for normal systolic wall thickening." Circ Res 77.1, pp. 182193.

23. MacGowan GA, Shapiro EP, Azhari H, Siu CO, Hees PS, Hutchins GM, Weiss JL, and Rademakers FE (1997). "Noninvasive measurement of shortening in the fiber and cross-fiber directions in the normal human left ventricle and in idiopathic dilated cardiomyopathy". Circulation 96 , pp. 535-541.

24. Matsumura H, Aizawa Y, and Kumaki K (1990). "Myocardial architecture in situs inversus vicerum totalis". In: Developmental cardiology: morphogenesis and function. Ed. by E Clark and A Takao. ISBN-13: 978-0-879-93382-1. Futura Pub Co, Mount Kisco, pp. 605-624.

25. Nielsen PM, LeGrice IJ, Smaill BH, and Hunter PJ (1991). "Mathematical model of geometry and fibrous structure of the heart". Am J Physiol - Heart Circ Physiol 260, H1365-H1378.

26. Nikolić S, Yellin EL, Tamura K, Vetter H, Tamura T, Meisner JS, and Frater RW (1988). "Passive properties of canine left ventricle: diastolic stiffness and restoring forces." Circ Res 62.6, pp. 1210-1222.

27. Omens JH, Usyk TP, Li Z, and McCulloch AD (2002). “Muscle LIM protein deficiency leads to alterations in passive ventricular mechanics." Am J Physiol Heart Circ Physiol 282.2, H680-H687.

28. Pluijmert M, Kroon W, Delhaas T, and Bovendeerd P (2012). "Adaptive reorientation of cardiac myofibers: the long-term effect of initial and boundary conditions". Mechanics Research Communications 42, pp. 60-67.

29. Rijcken JM, Bovendeerd PHM, Schoofs AJG, Van Campen DH, and Arts T (1999). "Optimization of cardiac fiber orientation for homogeneous fiber strain during ejection". Ann Biomed Eng 27, pp. 289-297.

30. Rodriguez EK, Omens JH, Waldman LK, and McCulloch AD (1993). "Effect of residual stress on transmural sarcomere length distributions in rat left ventricle". Am J Physiol - Heart Circ Physiol 264, H1048-H1056.

31. Schmid H, O'Callaghan P, Nash MP, Lin W, LeGrice IJ, Smaill BH, Young AA, and Hunter PJ (2008). "Myocardial material parameter estimation: a non-homogeneous finite element study from simple shear tests." Biomech Model Mechanobiol 7.3, pp. 161-173. 
32. Streeter DD and Hanna WT (1973). "Engineering mechanics for successive states in canine left ventricular myocardium." Circ Res 33.6, pp. 639-664.

33. Streeter Jr D, Spotnitz HM, Patel DP, Ross Jr J, and Sonnenblick EH (1969). "Fiber orientation in the canine left ventricle during diastole and systole." Circ Res 24.3, pp. 339-347.

34. Ubbink SWJ, Bovendeerd PHM, Delhaas T, Arts T, and Van de Vosse FN (2006). "Towards model-based analysis of cardiac MR tagging data: Relation between left ventricular shear strain and myofiber orientation". Med Imag Anal 10, pp. 623-641.

35. Yin FC, Strumpf RK, Chew PH, and Zeger SL (1987). "Quantification of the mechanical properties of noncontracting canine myocardium under simultaneous biaxial loading." $J$ Biomech 20.6, pp. 577-589. 
Chapter 3 


\section{Chapter 4}

\section{Effect of activation and triaxial active stress development}

The contents of this chapter are based on:

M. Pluijmert, P.H.M. Bovendeerd, W. Kroon, F.W. Prinzen, T. Delhaas, Effects of Activation Pattern and Active Stress Development on Myocardial Shear in a Model with Adaptive Myofiber Reorientation, American Journal of Physiology - Heart and Circulatory Physiology, 306(4):H538H546, 2014 


\section{Abstract}

It has been hypothesized that myofiber orientation adapts to achieve a preferred mechanical loading state in the myocardial tissue. Earlier studies tested this hypothesis in a combined model of left ventricular (LV) mechanics and remodeling of myofiber orientation in response to fiber cross-fiber shear, assuming synchronous timing of activation and uniaxial active stress development. Differences between computed and measured patterns of circumferential-radial shear strain $E_{c r}$ were assumed to be caused by limitations in either the LV mechanics model or the myofiber reorientation model. Therefore, we extended the LV mechanics model with a physiological transmural and longitudinal gradient in activation pattern and with triaxial active stress development. We investigated effects on myofiber reorientation, LV function, and deformation. The effect on the developed pattern of the transverse fiber angle $\alpha_{t, 0}$ and the effect on global pump function were minor. Triaxial active stress development decreased amplitudes of $E_{c r}$ towards values within the experimental range and resulted in a similar base-to-apex gradient during ejection in model computed and measured $E_{c r}$. Physiological pattern of mechanical activation resulted in better agreement between computed and measured strain in myofiber direction, especially during isovolumic contraction phase and first half of ejection. In addition, remodeling was favorable for LV pump and myofiber function. In conclusion, the outcome of the combined model of LV mechanics and remodeling of myofiber orientation is found to become more physiologic by extending the mechanics model with triaxial active stress development and physiological activation pattern. 


\subsection{Introduction}

Mathematical models of cardiac mechanics can be used to relate abnormal cardiac deformation, as measured noninvasively by ultrasound or magnetic resonance tagging (MRT), to the underlying pathology. As a first step towards this application, one would require the models to be able to correctly predict deformation in the wall of the healthy human heart. Though most finite element (FE) models are able to correctly predict circumferential, longitudinal and radial strains $[6,14,15,19,24,27]$, they are not capable to correctly predict the shearing pattern of the myocardium $[5,14,27]$ as measured in experiments $[2,18,21,26]$. Because myocardial shear is not kinematically coupled to the cavity volume, it would be a more sensitive measure to evaluate model results.

FE modeling results from our group indicate a strong relation between myofiber orientation and strain distribution in the LV wall, in particular shear strain. However, experimental data show that shear strain varies little in between healthy individuals [5], suggesting that variation of cardiac myofiber orientation in between individuals is low as well. Therefore, we hypothesized that myofiber orientation adapts to achieve a preferred mechanical loading state in the tissue [1] and that the myofiber orientation pattern in the normal adult heart can be seen as the result of a successful adaptation process. This hypothesis has been pushed further in a computational model study in which myofiber orientation was allowed to change in a response to mechanical load $[16,22]$. The myofiber orientation field predicted for a normal adult heart agreed favorably with experimental data. In addition, a significant increase in homogeneity of mechanical load was observed after reorientation, along with an increase in pump function. Finally, the level of shear strain was reduced to physiological levels, but differences remained between the patterns of predicted and measured shear strains.

We hypothesized that the differences between predicted and measured shear strains are caused by simplifications in the model of LV mechanics. The simplifications will not only have a direct effect on the deformation pattern of the myocardium, but also an indirect effect, since they affect the process of shear-induced myofiber reorientation. For example, earlier studies that used a perfectly synchronous onset of active stress development [15], also showed synchronous onset of myofiber shortening. However, electrical activation in a healthy heart takes 50-70ms and also onset of myofiber shortening has been shown to be asynchronous [3]. This suggests that active stress development is asynchronous as well. Asynchronous shortening induces shearing deformation of the myocardium and this in turn will affect the hypothesized myofiber reorientation mechanism.

Also, until now active stress was assumed to develop along the myofiber 
direction only. Experimentally it has been shown that a considerable component of active stress may be generated perpendicular to the myofiber direction [17]. Extension from uniaxial to triaxial active stress development in models of cardiac mechanics was found to lead to a reduction of shear amplitudes, due to an increase of transmural mechanical coupling [5, 27, 28].

Therefore, the aim of the current study was to investigate the effects of a physiological, slightly asynchronous, timing of activation and the effects of triaxial active stress development on myofiber reorientation, on LV global pump and on local myofiber function, and on shear deformation, as computed in a cardiac mechanics model with shear-induced myofiber reorientation [16].

\subsection{Methods}

\section{Model of left ventricular mechanics}

Tissue deformations during the cardiac cycle are calculated with a generic finite element (FE) model of LV mechanics (figure 4.1). With respect to geometry, material properties and the circulation in which the LV is embedded, this FE model is identical to the model presented in extenso in [5]. Characteristics important for this paper are repeated here for readability.

In the reference state, defined as the passive stress-free state, a thick-walled ellipsoidal geometry is assumed (figure 4.1A). During the cardiac cycle, myocardial tissue Cauchy stress $\sigma$ is composed of an active component $\sigma_{a}$ and a passive component $\sigma_{p}$ :

$$
\sigma=\sigma_{a}\left[\vec{e}_{f} \vec{e}_{f}+\beta\left(\vec{e}_{s} \vec{e}_{s}+\vec{e}_{n} \vec{e}_{n}\right)\right]+\sigma_{p}
$$

with $\vec{e}_{f}, \vec{e}_{s}$, and $\vec{e}_{n}$ the unit vectors in the current myofiber, sheet and sheetnormal direction in the deformed tissue, respectively. Active stress $\sigma_{a}$ is modeled through a series arrangement of a contractile and a series elastic element. The parameter $\beta$ describes the level of active stress development in the crossfiber direction. The magnitude of $\sigma_{a}$ depends on time elapsed since activation, sarcomere length and sarcomere shortening velocity [15]. Active stress development is initiated with a cycle time of $800 \mathrm{~ms}$. Passive material behavior is assumed nonlinearly elastic, transversely isotropic, and nearly incompressible [5].

In the model, the quasi-static equations of conservation of linear momentum are solved. At the base, essential boundary conditions are defined to suppress rigid body motion and to represent the mechanical effect of structures, e.g. the valvular annulus, left out of the model. In total, the LV wall is represented by 30 triquadratic hexahedral finite elements: 6 elements in radial, 1 in circumferential and 5 in longitudinal direction [23]. Left ventricular 
pressure $p_{l v}$ is determined from the interaction of the $\mathrm{LV}$ with the circulation according to a lumped parameter model [5] (figure 4.1A).

\section{Model of adaptive myofiber orientation}

As opposed to myofiber orientation in the current loaded state, denoted by $\vec{e}_{f}$, myofiber orientation in the mechanically unloaded state is denoted by $\vec{e}_{f, 0}$. In this unloaded state, myofiber orientation is defined with respect to a local cardiac coordinate system $\left\{\vec{e}_{t, 0}, \vec{e}_{l, 0}, \vec{e}_{c, 0}\right\}$ (figure 4.1B). The transmural direction $\vec{e}_{t, 0}$ is defined as the outer normal to the cardiac surfaces. The longitudinal direction $\vec{e}_{l, 0}$ is defined perpendicular to $\vec{e}_{t, 0}$ from apex to base. To obtain a right-handed coordinate system, the circumferential direction $\vec{e}_{c, 0}$ is defined in clockwise direction when viewing the LV in apex-to-base direction.

In the local cardiac coordinate system, myofiber orientation is quantified by two angles. The helix angle $\alpha_{h, 0}$ is defined as the angle between $\vec{e}_{c, 0}$ and the projection of $\vec{e}_{f, 0}$ on the circumferential-longitudinal plane $\left(\vec{e}_{c, 0}, \vec{e}_{l, 0}\right)$. The transverse angle $\alpha_{t, 0}$ is defined as the angle between $\vec{e}_{c, 0}$ and the projection of $\vec{e}_{f, 0}$ on the circumferential-transmural plane $\left(\vec{e}_{c, 0}, \vec{e}_{t, 0}\right)$.

Myofiber orientation in the unloaded state is subject to remodeling, causing a structural reorientation of the myofiber that is quantified by a change in $\vec{e}_{f, 0}$, and, consequently, a change in $\alpha_{h, 0}$ and $\alpha_{t, 0}$. The reorientation process is simulated with the model by Kroon et al. [16]. In this model, it was assumed that structural changes in myofiber orientation occur due to damage and repair of the connections between extra-cellular matrix and myofibers (figure 4.2). The conceptual model was translated into a mathematical model in which the myofiber orientation in the unloaded state $\vec{e}_{f, 0}$ will evolve towards the myofiber orientation in the loaded state corrected for rigid body rotation $\vec{e}_{f}^{*}$ :

$$
\frac{\partial \vec{e}_{f, 0}}{\partial t}=\frac{1}{\kappa}\left(\vec{e}_{f}^{*}-\vec{e}_{f, 0}\right)
$$

with $\kappa$ the adaptation time constant, which is set to $3200 m s . \vec{e}_{f}^{*}$ was derived from the actual myofiber orientation in the deformed tissue $\vec{e}_{f}$ as follows:

$$
\vec{e}_{f}=\frac{\boldsymbol{F} \cdot \vec{e}_{f, 0}}{\lambda_{f}}=\frac{\boldsymbol{R} \cdot \boldsymbol{U} \cdot \vec{e}_{f, 0}}{\lambda_{f}}=\boldsymbol{R} \cdot \vec{e}_{f}^{*} ; \quad \lambda_{f}=\left|\boldsymbol{U} \cdot \vec{e}_{f, 0}\right|
$$

with $\lambda_{f}$ the myofiber stretch ratio and $\boldsymbol{F}$ the deformation tensor that consists of the actual deformation $U$ and rigid body rotation $R$. Since rigid body rotations are not sensed by the tissue, they are considered irrelevant to adaptation. At the endo- and epicardium, the adapted fiber orientation was forced to be parallel with the endo- and epicardial surfaces, respectively, to ensure myofibers do not stick out of these surfaces. 


\section{Simulations performed}

Four simulations of LV wall mechanics and myofiber reorientation were performed (table 4.1). Simulation $U N I+S Y N C$ is the reference simulation with uniaxial stress development $(\beta=0)$ and synchronous development of active stress throughout the LV wall. In simulation $T R I+S Y N C, \beta$ in (5.3) is set to 0.15 , which indicates triaxial active stress development. The level of active stress in cross-fiber direction is equal to $15 \%$ of the level in myofiber direction. In simulation UNI+PHYS, active stress development was uniaxial, but the moment of onset of active stress development was not synchronous. Instead, a delay in transmural direction from endo- to epicardium and a delay in longitudinal direction from apex to base of both $25 \mathrm{~ms}$ were introduced, resulting in a more physiological pattern of activation with a total activation time of $50 \mathrm{~ms}$ for the total LV (figure 4.1C). The combination of including triaxial active stress development and physiological onset of active stress development was simulated in TRI+PHYS.

In all simulations, the initial distribution of the helix angle $\alpha_{h, 0}$ is described by the parameterized distribution in [5]. It varies nonlinearly with the transmural position from endocardium to epicardium (figure 4.3, left). The initial condition for transmural distribution of $\alpha_{t, 0}$ is set to zero.

The first 10 consecutive cardiac cycles were used to reach a hemodynamic steady state and myofiber reorientation was disabled. In subsequent cycles myofiber orientation was adapted per node and the parameterized description of fiber orientation was abandoned. A total of 10 adaptation cycles was simulated, which corresponds with 40 cardiac cycles. The new LV structure was analyzed in terms of the adapted values of $\alpha_{h, 0}$ and $\alpha_{t, 0}$. LV global function was quantified through maximum LV pressure $p_{l v, \text { max }}$, stroke volume $S V$, and stroke work $W_{\text {stroke }}$. Since these global function parameters depend on LV filling pressure, and filling pressure after adaptation differed in between simulations, these parameters were determined from a new simulation at a constant preload of $p_{\text {ven }}=1.8 \mathrm{kPa}$. LV local function was quantified by local myofiber function, expressed through maximum myofiber stress $\sigma_{f, \max }$, and natural myofiber strain during ejection $\epsilon_{f, e j}$, during isovolumic contraction (IC) $\epsilon_{f, i c}$, and during isovolumic relaxation (IR) $\epsilon_{f, i r}$.

Evaluation of the models was performed by comparing predicted fiber orientation and shear strain with experimental data. We used circumferential-radial shear strain data from our own group [5] $(n=3)$, extended with data from the original experiment [8] $(n=9)$. As heart rate varies between subjects, we corrected for differences in duration of the ejection and the filling phase. For determination of the Green-Lagrange strain tensor component $E_{c r}$ from MRT measurements, we refer to [5]. Computed $E_{c r}$ in the final state (after 10 adaptation cycles) was compared with the experimental average $E_{c r}$. In 
A

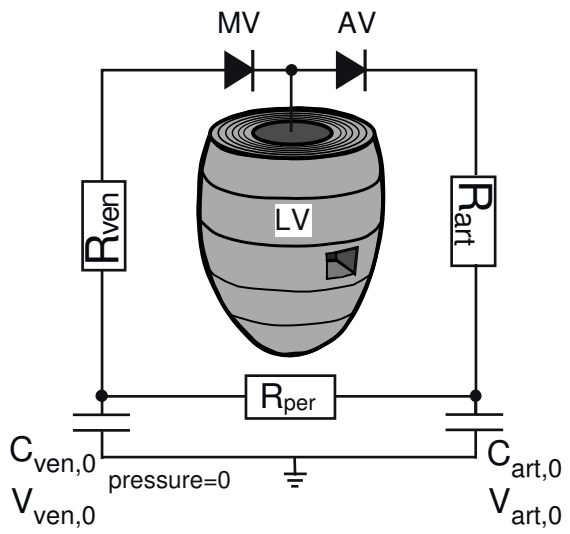

B

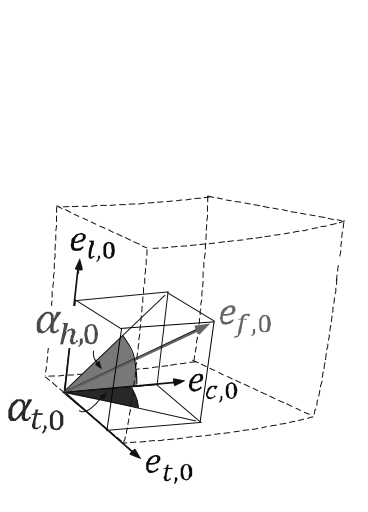

C

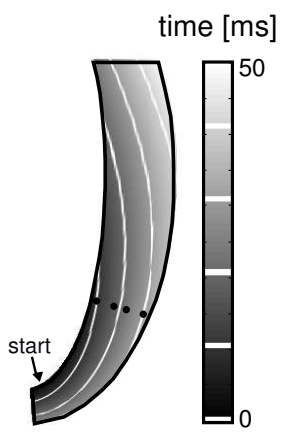

Figure 4.1: Finite element (FE) model of left ventricular (LV) mechanics. A The ellipsoidally shaped FE mesh of the LV consists of 30 elements and is incorporated in a lumped parameter model of the circulation. AV, aortic valve; $C_{a r t}$, arterial compliance; $C_{v e n}$, venous compliance; $\mathrm{MV}$, mitral valve; $R_{\text {art }}$, arterial resistance; $R_{\text {per }}$, peripheral resistance; $R_{v e n}$, venous resistance. B Description of myofiber orientation vector in the unloaded state $\vec{e}_{f, 0}$ by helix angle $\alpha_{h, 0}$ and transverse angle $\alpha_{t, 0}$ using a local cardiac coordinate system $\left\{\vec{e}_{l, 0}, \vec{e}_{t, 0}, \vec{e}_{c, 0}\right\}$. C Mechanical activation pattern as simulated in UNI+PHYS and TRI+PHYS. The pattern is based on measurements from Durrer et al. [10]. Black dots indicate four of the ten locations of the beads from the experiments of Ashikaga et al. [3].

addition, we compared predicted strain with data from other experimental studies [18, 29].

\subsection{Results}

Figure 4.3 shows the structural changes after 10 adaptation cycles in simulations UNI+SYNC,UNI+PHYS, TRI+SYNC, and TRI+PHYS. In all simulations, major characteristics of the transmural distribution of the helix angle $\alpha_{h, 0}$ remained. The transverse angle $\alpha_{t, 0}$ developed a distribution with negative values at the apex and positive values at the base. The pattern is similar in all simulations, but angles are larger with uniaxial (UNI+SYNC and UNI+PHYS) than with triaxial (TRI+SYNC and TRI+PHYS) active stress development, especially towards the endocardial apex.

In all simulations, global LV function improved significantly during reorientation as indicated by the increase in maximum left ventricular pressure $p_{l v, \text { max }}$, stroke volume $S V$, and stroke work $W_{\text {stroke }}$ (table 4.2). After 10 adaptation cycles, all these parameters are largest in UNI+SYNC $(17.2 \mathrm{kPa}, 65.6 \mathrm{ml}$, and $1.01 \mathrm{~J}$, respectively), but differences between simulations are small. 


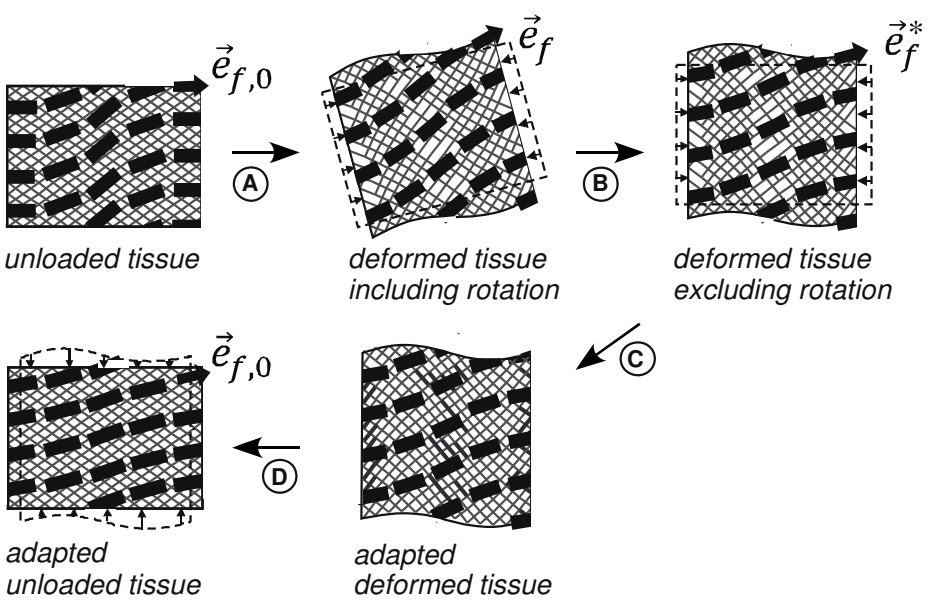

Figure 4.2: Conceptual model on the hypothesis of myofiber reorientation adapted from [16] and expressed in equation 5.1. In the unloaded configuration, the myofiber orientation vector is denoted by $\vec{e}_{f, 0}$. During a cardiac cycle, tissue deformations take place (A). The myofiber orientation vector in the deformed configuration is denoted by $\vec{e}_{f}$. Shear forces as a result of these deformations are assumed to damage the connections between extra-cellular matrix (ECM, raster) and myofibers (thick black lines). Rigid body rotations are not sensed by the tissue and therefore considered irrelevant to adaptation (B). Here, myofiber orientation is denoted by $\vec{e}_{f}^{*}$. Due to tissue turnover, new connections between the ECM and the myofibers are formed continuously $(\mathrm{C})$. When a connection is made, myofibers tend to be fixed within the tissue (D). This results in an adapted myofiber orientation in the unloaded state.

Table 4.1: Overview of the four simulations performed characterized by two settings of active stress development, uniaxial or triaxial, and two settings of timing of onset of active stress development, synchronous or physiological.

\begin{tabular}{cc||cc}
\hline & & \multicolumn{2}{c}{ active stress development } \\
& & uniaxial & triaxial \\
\hline \hline & & & \\
$\begin{array}{c}\text { onset } \\
\text { active stress } \\
\text { development }\end{array}$ & phynchronous & phI+SYNC & TRI+SYNC \\
\hline
\end{tabular}


Local LV function also improved during reorientation as indicated by the absolute increase of maximum myofiber stress $\sigma_{f, \max }$ and myofiber strain during ejection $\epsilon_{f, e j}$ (table 4.3). Simulation UNI+SYNC not only showed largest values for global parameters, but also for local parameters $\sigma_{f, \max }(42.1 \pm 5.2$ $\mathrm{kPa})$ and $\epsilon_{f, e j}(-0.164 \pm 0.012)$.

Absolute values of myofiber strains during the isovolumic phases, $\epsilon_{f, i c}$ and $\epsilon_{f, i r}$, decreased significantly as a result of reorientation (table 4.3). In simulations with physiological onset of active stress development (UNI+PHYS and TRI+PHYS) absolute myofiber strain during IC $\epsilon_{f, i c}$ was larger and less homogeneous, and absolute myofiber strain during ejection $\epsilon_{f, e j}$ was smaller and less homogeneous than in simulations with synchronous onset of active stress development $U N I+S Y N C$ and TRI+SYNC. Myofiber strain during IR $\epsilon_{f, i r}$ was smallest with triaxial active stress development and synchronous timing of activation (TRI+SYNC). Standard deviations were also smallest in simulation TRI+SYNC. Introducing physiological onset of active stress development induced inhomogeneity as reflected by the increased standard deviation in simulation TRI+PHYS.

The results of $E_{c r}$ are presented in figure 4.4. Simulation results are presented in the four graphs on the left. On the top right, averaged $E_{c r}$ (mean+std) of nine healthy subjects is shown. Both simulated and experimental strains are computed with respect to begin-ejection and averaged per MR-slice in circumferential and radial direction. In the cross-section on the bottom right, MR-slices relative to the model geometry are shown.

$E_{c r}$ amplitudes were smaller with triaxial (TRI+SYNC and TRI+PHYS) than with uniaxial (UNI+SYNC and UNI+PHYS) active stress development. When including physiological onset of active stress development (UNI+PHYS and $T R I+P H Y S), E_{c r}$ amplitudes increased. In addition, the simulations with triaxial active stress development showed a more realistic base-to-apex gradient of $E_{c r}$ during ejection. With uniaxial active stress development, the base-to-apex gradient of $E_{c r}$ only resembled experimental results at end ejection. Amplitudes of $E_{c r}$ exceeded experimental amplitudes with uniaxial active stress development (UNI+SYNC and UNI+PHYS). The combination of triaxial active stress and physiological activation pattern (TRI+PHYS) brought values and base-to-apex gradient of $E_{c r}$ in more agreement with experimental results.

To further test the match between computed and experimental strains, we collected strain data from MR tagging [18, 29] and biplane radiography $[2,4]$ studies in table 4.4. In both simulations and measurements, values of normal strains were larger than shear strains. Also, variation of normal strains in between simulations was far less than variation of shear strains. In general, the normal strains lay well within the experimental range. Only radial strain $E_{r r}$ was larger in the simulations. Near the equator (eq), shear strains also fell within the experimental range. In the apical region (apex), only shear strain 


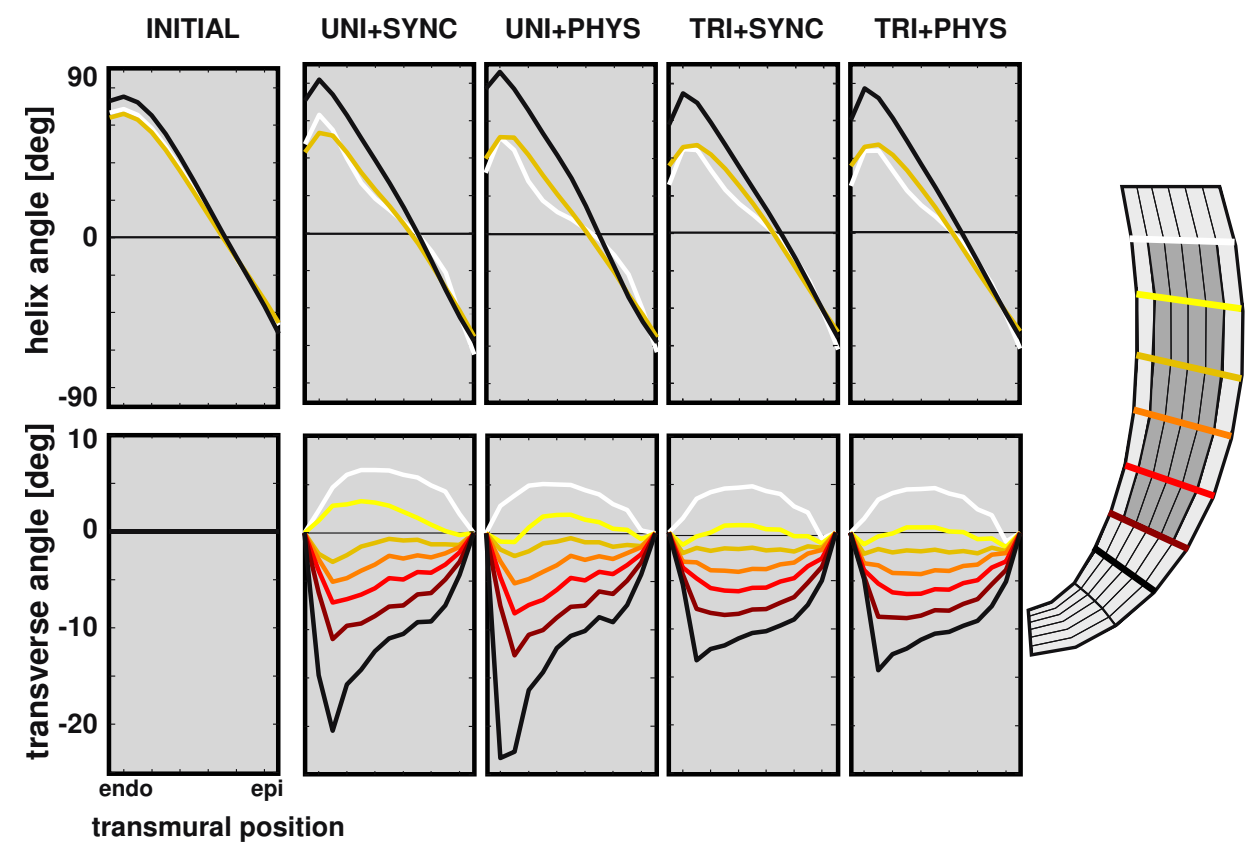

Figure 4.3: Helix $\alpha_{h, 0}$ and transverse $\alpha_{t, 0}$ fiber angles. On the left side, the initial distributions of $\alpha_{h, 0}$ and $\alpha_{t, 0}$ are presented. Thereafter, results of the simulations $U N I+S Y N C, U N I+P H Y S, T R I+S Y N C$, and TRI+PHYS are shown after 10 adaptation cycles. Thin lines represent the initial distribution. Adaptation cycle is defined as time $t$ normalized with respect to adaptation time constant $\kappa(\mathrm{t} / \kappa)$. Numbers refer to levels between base and apex as indicated in the cross-section on the right. The helix angle is shown for levels 1,3 , and 7 .

values in simulation $U N I+P H Y S$ deviated from experimental values. When including triaxial active stress development (TRI+PHYS), shear strain values lay in the experimental range.

In the left four panels of figure 4.5, myofiber strain at 4 transmural locations (figure $4.1 \mathrm{C}$ ) is shown for the simulations during a cardiac cycle after 10 adaptation cycles. With synchronous onset of active stress development, myofibers at the epicardium started to shorten while endocardial myofibers were initially stretched. When onset of active stress development is more physiological, endocardial fibers started to shorten first at the expense of stretching epicardial fibers. Results with physiological activation PHYS resembled experimental data from Ashikaga et al. [3] better (figure 4.5, right). 

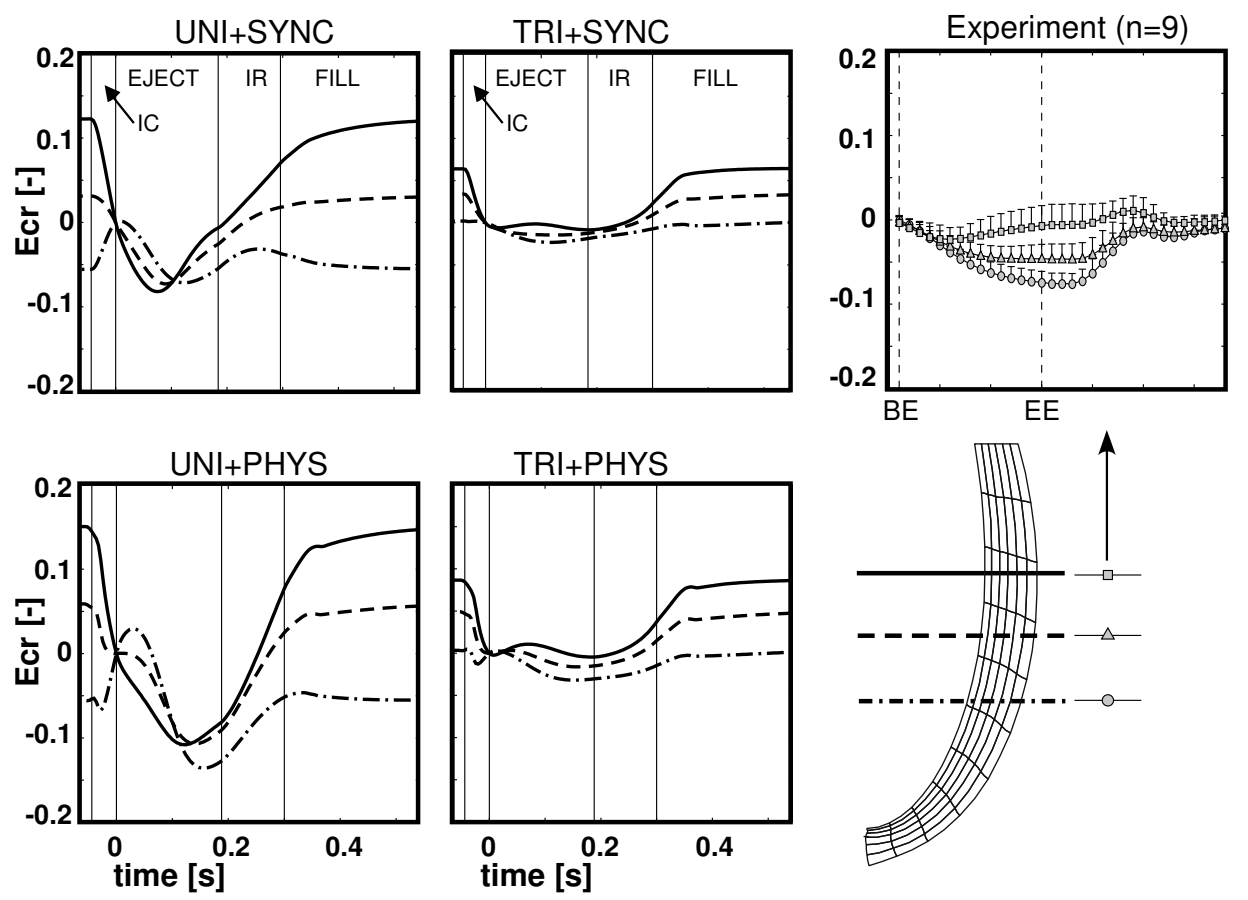

Figure 4.4: Results of circumferential-radial shear $E_{c r}$ after 10 adaptation cycles from the four simulations defined in table 4.1 (left). IC=isovolumic contraction phase; $E J E C T=$ ejection phase; $I R=$ isovolumic relaxation phase; $F I L L=$ filling phase. The location of the MR-slices relative to the model geometry at begin-ejection is shown on the bottom right. On the top right, average $E_{c r}$ from nine healthy subjects as measured with MRT [8] are shown. Begin-ejection $(B E$, time $=0)$ is the reference state and $E_{c r}$ is averaged in circumferential and transmural direction.

Table 4.2: Values of global pump parameters maximum LV pressure $p_{l v, \max }$, stroke volume $S V$, and stroke work $W_{\text {stroke }}$ before (initial) and after 10 adaptation cycles (final). Pump parameters were determined in a simulation where myofiber reorientation was not allowed and with a constant preload of $1.8 \mathrm{kPa}$.

\begin{tabular}{lcccccc}
\hline & \multicolumn{6}{c}{ global } \\
& \multicolumn{2}{c}{$p_{l v, \max }[\mathrm{kPa}]$} & \multicolumn{2}{c}{$S V[\mathrm{ml}]$} & \multicolumn{2}{c}{$W_{\text {stroke }}[\mathrm{J}]$} \\
simulation & initial & final & initial & final & initial & final \\
\hline \hline UNI+SYNC & 15.6 & 17.2 & 57.8 & 65.6 & 0.79 & 1.01 \\
TRI+SYNC & 15.6 & 16.8 & 57.8 & 63.4 & 0.79 & 0.95 \\
UNI+PHYS & 15.4 & 16.9 & 57.2 & 64.1 & 0.76 & 0.96 \\
TRI+PHYS & 15.9 & 16.8 & 58.6 & 62.3 & 0.82 & 0.93 \\
\hline
\end{tabular}



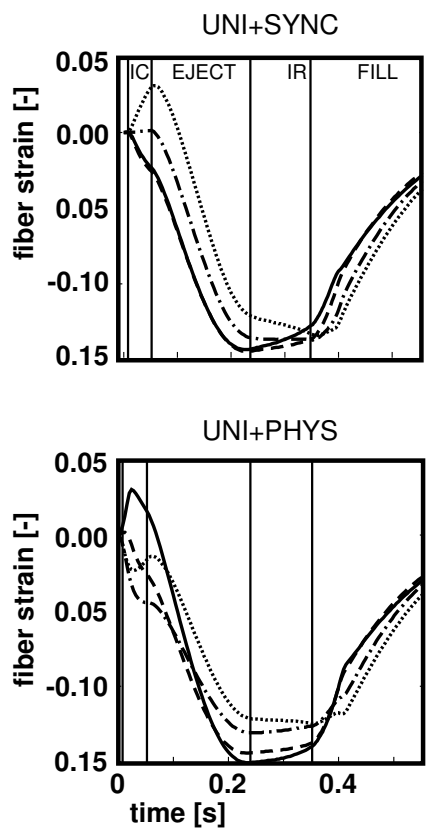

TRI+SYNC

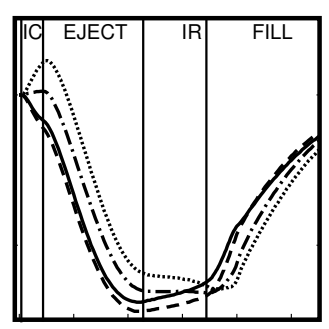

TRI+PHYS

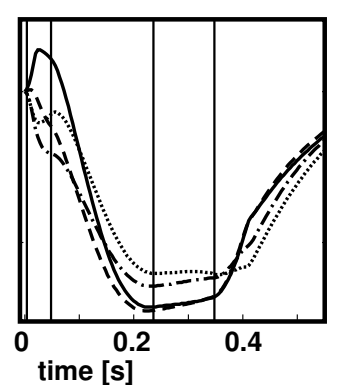

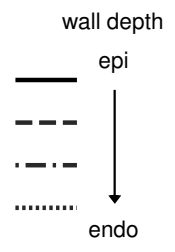

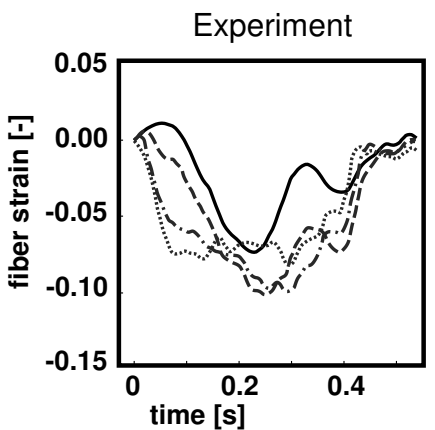

Figure 4.5: Left: Fiber strain $E_{f}$ after 10 adaptation cycles in the four simulations. Linestyles refer to the location between the epicardial and endocardial surface (figure $4.1 \mathrm{C}$ ), assumed to resemble four locations of the original experimental results of [3]. Right: Fiber strains that are measured using transmural bead markers in dogs in vivo (taken from Ashikaga et al. [3]). In the experiment, fiber strains were measured at 10 locations between epi- and endocardium. Here, results are shown at $10 \%$ (solid), $40 \%$ (dashed), $60 \%$ (dashed-dotted), and 90\% (dotted) of the measured wall depth. In both figures, fiber strains are computed with respect to the first moment of onset of myofiber shortening. 

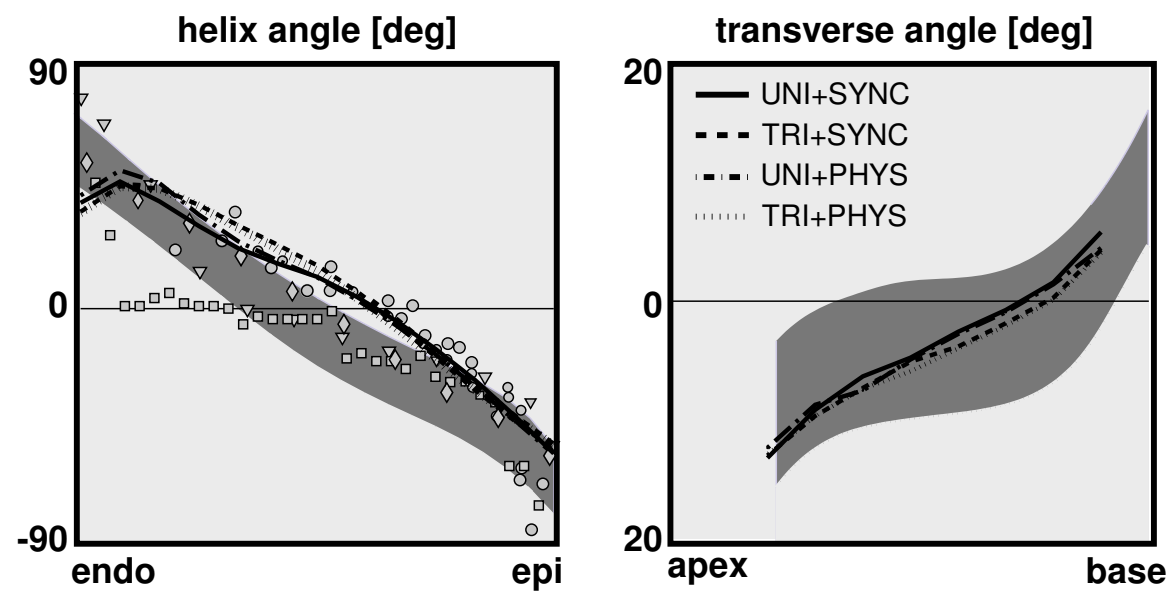

Figure 4.6: Comparison of predicted fiber angles in the four simulations after 10 adaptation cycles with experimental data. Left : Experimental data on the helix angle $\alpha_{h, 0}$ at equatorial level from [3] (diamonds), [11] (band), [13] (squares), [20] (circles), and [25] (triangles). Simulation results at level 2 (see figure 4.3) are shown to represent equatorial level. Right : Experimental data on the transverse angle $\alpha_{t, 0}$ at mid-wall [11]. As in the experiment, the mid-wall region in the mesh was defined as the transmural region with a helix angle close to $0^{\circ}$.

Table 4.3: Values of local tissue loading parameters maximum myofiber stress $\sigma_{f, \max }$, and natural myofiber strain during ejection $\epsilon_{f, e j}, \mathrm{IC} \epsilon_{f, i c}$, and IR $\epsilon_{f, i r}$ before (initial) and after 10 adaptation cycles (final). Local function parameters are presented by mean \pm std calculated from the grey area in Fig 4.2, bottom right.

\begin{tabular}{lcccc}
\hline & \multicolumn{2}{c}{$\sigma_{f, \max }[\mathrm{kPa}]$} & \multicolumn{2}{c}{ local } \\
simulation & initial & final & initial & $\epsilon_{f, e j}[-]$ \\
final \\
\hline \hline UNI+SYNC & $35.6 \pm 8.7$ & $42.1 \pm 5.2$ & $-0.141 \pm 0.014$ & $-0.164 \pm 0.012$ \\
TRI+SYNC & $36.5 \pm 6.1$ & $38.9 \pm 4.7$ & $-0.141 \pm 0.012$ & $-0.160 \pm 0.012$ \\
UNI+PHYS & $33.6 \pm 10.0$ & $37.7 \pm 7.9$ & $-0.103 \pm 0.038$ & $-0.138 \pm 0.043$ \\
TRI+PHYS & $33.8 \pm 7.0$ & $35.7 \pm 7.1$ & $-0.112 \pm 0.035$ & $-0.135 \pm 0.038$ \\
\hline \multicolumn{4}{c}{$\epsilon_{f, i c}[-]$} & \multicolumn{4}{c}{$\epsilon_{f, i r}[-]$} \\
simulation & initial & final & initial & final \\
\hline \hline UNI+SYNC & $-0.038 \pm 0.015$ & $-0.018 \pm 0.010$ & $0.027 \pm 0.010$ & $0.011 \pm 0.007$ \\
TRI+SYNC & $-0.028 \pm 0.011$ & $-0.015 \pm 0.008$ & $0.019 \pm 0.006$ & $0.010 \pm 0.007$ \\
UNI+PHYS & $-0.067 \pm 0.039$ & $-0.038 \pm 0.022$ & $0.032 \pm 0.018$ & $0.018 \pm 0.008$ \\
TRI+PHYS & $-0.054 \pm 0.035$ & $-0.038 \pm 0.025$ & $0.026 \pm 0.015$ & $0.016 \pm 0.007$ \\
\hline
\end{tabular}


Table 4.4: End-ejection strain values computed from the simulation results after 10 adaptation cycles. Strains were taken near the midwall, at $55 \%$ (eq) and at $30 \%$ (apex) of the distance from the apex to the basal plane with the reference at begin-ejection. Experimental data in humans were taken from Moore et al. [18] and Young et al. [29] and are presented as mean (std).

\begin{tabular}{|c|c|c|c|c|c|c|c|c|}
\hline \multicolumn{3}{|c|}{$\begin{array}{l}\operatorname{sim}+\exp \rightarrow \\
\text { strain } \downarrow\end{array}$} & $\begin{array}{l}\text { UNI+ } \\
\text { SYNC }\end{array}$ & $\begin{array}{l}\text { TRI+ } \\
\text { SYNC }\end{array}$ & $\begin{array}{l}\text { UNI+ } \\
\text { PHYS }\end{array}$ & $\begin{array}{l}\text { TRI+ } \\
\text { PHYS }\end{array}$ & $\begin{array}{l}\text { Moore } \\
2000 \text { [18] }\end{array}$ & $\begin{array}{l}\text { Young } \\
1994 \text { [29] }\end{array}$ \\
\hline \multirow{6}{*}{ 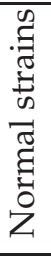 } & \multirow[t]{2}{*}{ 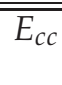 } & $\overline{\text { eq }}$ & 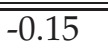 & 年-0.16 & $\begin{array}{c}-0.16 \\
\end{array}$ & 年-0.16 & $\overline{-0.22(0.03)}$ & $\overline{c-0.21(0.02)}$ \\
\hline & & apex & -0.15 & -0.15 & -0.18 & -0.14 & $-0.24(0.04)$ & $-0.22(0.02)$ \\
\hline & \multirow{2}{*}{$E_{r r}$} & eq & 0.54 & 0.44 & 0.59 & 0.46 & $0.38(0.18)$ & $0.21(0.10)$ \\
\hline & & apex & 0.51 & 0.43 & 0.46 & 0.37 & $0.49(0.29)$ & $0.10(0.06)$ \\
\hline & \multirow[t]{2}{*}{$E_{l l}$} & eq & -0.15 & -0.12 & -0.15 & -0.13 & $-0.14(0.04)$ & $-0.16(0.03)$ \\
\hline & & apex & -0.15 & -0.12 & -0.13 & -0.11 & $-0.19(0.03)$ & $-0.18(0.03)$ \\
\hline \multirow{6}{*}{ 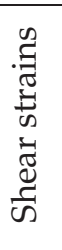 } & \multirow[t]{2}{*}{$\overline{E_{c r}}$} & $\overline{\mathrm{eq}}$ & 0.08 & $\overline{c 0.01}$ & 0.08 & $\begin{array}{l}-0.02 \\
\end{array}$ & $0.05(0.06)$ & $-0.03(0.02)$ \\
\hline & & apex & 0.13 & 0.03 & 0.19 & 0.03 & $0.05(0.08)$ & $-0.05(0.03)$ \\
\hline & \multirow[t]{2}{*}{$E_{l r}$} & eq & 0.03 & 0.01 & 0.08 & 0.04 & $0.07(0.06)$ & $0.02(0.02)$ \\
\hline & & apex & 0.07 & -0.01 & 0.13 & 0.03 & $0.05(0.08)$ & $0.00(0.04)$ \\
\hline & \multirow{2}{*}{$E_{c l}$} & eq & 0.03 & 0.03 & 0.08 & 0.08 & $0.03(0.04)$ & $-0.03(0.01)$ \\
\hline & & apex & 0.06 & -0.01 & 0.09 & 0.04 & $0.04(0.03)$ & $-0.01(0.02)$ \\
\hline
\end{tabular}

\subsection{Discussion}

In this study, we investigated the effects of uniaxial versus triaxial active stress development and of a synchronous versus a physiological activation pattern on myofiber reorientation, LV function and shear deformation in a combined model of LV mechanics with shear-induced myofiber reorientation. The effect on the developed pattern of the transverse fiber angle $\alpha_{t, 0}$ and the effect on global pump function is minor. Patterns of circumferential-radial shear $E_{c r}$ differ between the simulations. Triaxial active stress development decreases amplitudes of $E_{c r}$ towards values that lie in the experimental range and results in a similar base-to-apex gradient during ejection in model computed and measured $E_{c r}$. Physiological pattern of mechanical activation resulted in better agreement between computed and measured myofiber strain, especially during isovolumic contraction phase and first half of ejection. Combining physiological sequence of activation and triaxial active stress development improves agreement between computed and measured $E_{c r}$ and myofiber strain.

In this study, we especially focussed on $E_{c r}$ to make the comparison between computed and experimental results, because our own experimental data contain time courses of $E_{c r}$ in several levels between apex and base. This makes it possible to compare patterns and gradients during the cardiac cycle. According to table 4.4, $E_{c r}$ values differed in between simulations, but all fell 
well within the wide range of available experimental data. Figure 4.4 showed that the range of $E_{c r}$ during a cardiac cycle was too large in simulations with uniaxial active stress development when compared the experimental average.

In addition, introduction of triaxial stress development reduced the amplitudes of $E_{c r}$ during isovolumic contraction (IC) to about 0.10 (figure 4.4). Data during IC are not available from MR tagging experiments [18, 29]. MR tagging images are constructed over a series of cardiac cycles. To avoid blurring of the images because of variations in cycle length that predominantly occur as variations in the duration of diastole, the reference frame is generally made at begin-ejection. Because the MR signal decreases over time due to relaxation of the magnetization in the tissue, data on $E_{c r}$ during IC are not available. Nevertheless, the experimental results in figure 4.4 suggested that $E_{c r}$ was small during IC, because the signals went back to zero. Strain can also be measured by biplane radiography of lead beads implanted in the LV wall. In these experiments end-diastole is usually taken as the reference. The experiments of Ashikaga et al. [2,4] show that deformation occurs during IC, but with amplitudes not statistically different from zero, as is suggested also by the MRT results (figure 4.4). Thus, even the amplitude of $\sim 0.10$ in simulations with triaxial stress development is too high.

The finding that triaxial active stress development decreases $E_{c r}$ amplitudes is in line with results from our previous study [5]. It is explained by the fact that the LV wall is stiffer in cross-fiber direction during the active phase of the cardiac cycle. Less shearing deformation results in a smaller difference between the unloaded myofiber orientation $\vec{e}_{f, 0}$ and the actual myofiber orientation corrected for rigid body rotation $\vec{e}_{f}^{*}$ in equation 5.1. Consequently, the amplitude of myofiber reorientation during the cardiac cycle is smaller and fiber reorientation due to long term remodeling is reduced as well. This effect is reflected by the smaller values of the transverse angle $\alpha_{t, 0}$ in simulations triaxial compared to uniaxial active stress development (figure 4.3).

Remarkably, triaxial active stress development was able to keep $E_{c r}$ values during ejection in the experimental range under physiological sequence of activation (figure 4.4), a condition known to create significant transmural strain differences especially during isovolumic contraction (IC) ([3], figure 4.5). The increased shearing deformation during IC with physiological activation pattern did not result in larger values of $\alpha_{t, 0}$, because myofiber reorientation takes place during the whole cardiac cycle.

Comparison with experimental data $[18,29]$ on other (shear) strain components is performed in table 4.4. In all simulations, radial strain $E_{r r}$ was larger when compared to the experimental results. The experimental results showed a coefficient of variation on the order of $50 \%$, which indicates that $E_{r r}$ cannot be measured as accurately as the other two normal strain components $E_{c c}$ and 
$E_{l l}$. The coefficients of variation of $E_{c c}$ and $E_{l l}$ varied between $10 \%$ and $30 \%$. The small variation in between simulations of both normal strains and global function showed that normal strains are kinematically coupled to the cavity volume. The effects of triaxial active stress development and physiological activation pattern were hardly reflected in the normal strains.

In contrast, shear strain values differed more in between simulations. It indicates that shear strains are a more sensitive measure for evaluating model results. However, the small values and the large variation (coefficients of variation up to and over $100 \%$ ) of shear strains makes it more difficult to accurately measure them. Nevertheless, from table 4.4 we concluded that a physiological activation pattern altered shear strains when compared to a synchronous activation pattern. Including triaxial active stress development decreased shear strain values. Especially shear strain values in the apical region showed more agreement with experimental data when active stress development was triaxial.

In the present study, $\beta$ was set to 0.15 , which means that the amount of active stress in cross-fiber direction was set to equal $15 \%$ of the amount of active stress generated along the myofiber direction. In [5], it was shown that setting $\beta$ to 0.25 and excluding a transverse component in myofiber orientation decreased $E_{c r}$ to realistic values when compared to measured $E_{c r}$. However, a $\beta$ of 0.25 in combination with a transverse component in myofiber orientation that is measured in experiments [11], led to unrealistically small values. We performed additional simulations in which $\beta$ was varied between 0 and 0.2. A higher $\beta$ led to lower amplitudes of $\alpha_{t, 0}$, in agreement with the trend observed when comparing simulations UNI and TRI in figure 4.3. In addition, amplitudes of $E_{c r}$ got smaller when $\beta$ was higher. Finally, we chose to set $\beta$ to 0.15 in simulations TRI+SYNC and TRI+PHYS, because this resulted in $E_{c r}$ values that are in the experimental range. Experimental results that were obtained in steady-state barium contracture, indicate that the amount of active cross-fiber stress is in the range of $40 \%$ [17]. However, it still remains unknown to what extent these results are representative for the situation in the normal beating heart since no further experimental studies have yet been performed.

We used the simple first order equation 5.1 with time constant $\kappa$ in the order of seconds to model the reorientation process. The value we chose for $\kappa$ is much lower than the half-life of ECM collagen or than the time myocytes need to replace the bulk of their internal proteins. However, we did not aim to realistically model the time course of remodeling. Instead, we lowered $\kappa$ to a value that significantly decreased simulation time but had no effect on the final remodeled state.

Maximum LV pressure $p_{l v, \max }$, stroke volume $S V$, and stroke work $W_{\text {stroke }}$ were chosen to quantify global function. Because in a closed loop circulation model, these variables depend on the preload of the LV, we ran simulations 
with constant preload after adaptation to determine global function. The improvement of global function with respect to the initial configuration in all simulations (table 4.2) shows that reorientation, in particular the development of a transverse component in myofiber orientation, is favorable for LV pump function. The small differences in global parameters between the four simulations in their final configuration (table 4.2) indicate that global function was similar.

Restructuring of the LV wall was also favorable for local function, as indicated by the increased local function parameters in table 4.3 with respect to the initial configuration. The decrease in myofiber shortening and lengthening during isovolumic phases indicate a reduction of shearing deformation and an improvement of local myofiber function. These strains do not contribute to changes in cavity volume as the cavity volume is constant in those phases of the cardiac cycle.

Reorientation led to an increase in homogeneity of local myofiber function as indicated by the decreased standard deviations after reorientation (table 4.3). The development of a transverse component in myofiber direction increases mechanical coupling in the transverse direction and therefore, the tissue becomes less anisotropic. Triaxial active stress development also increases mechanical coupling in the transverse direction, which is seen in the smaller standard deviations in simulations with TRI compared to UNI. Nevertheless, complete homogeneity is unrealistic because electrical activation induces inhomogeneities (figure 4.5).

Limitations and recommendations The good match between myofiber strains from the experiment and myofiber strains computed with a physiological sequence of activation was especially present during IC and during the first half of the ejection phase (figure 4.5). Presumably, the match between simulation and experiment will improve by taking into account the experimental finding that relaxation in the endocardium is delayed [3].

The best way to evaluate the model is to compare myofiber orientations predicted by the model with myofiber orientations obtained from experiments. Unfortunately, the accuracy of current measurement techniques, such as magnetic resonance diffusion tensor imaging, is too low to prefer one model solution over the others, see figure 4.6. Regarding the strong relation between myofiber orientation and deformation, a next best option is to evaluate the model indirectly by comparing computed and measured modes of deformation.

The results of the shear-induced myofiber reorientation may have been influenced by the absence of sheets in the transversely isotropic constitutive model. The organization of myocytes in sheets [7] explains the more orthotropic 
behavior observed in simple shear experiments [9]. Extension of the adaptation model by including sheets and reorientation thereof can be a next step.

Geometry and structure of the LV were assumed rotationally symmetric and the interaction of the LV with the right ventricle (RV) was not taken into account. The mechanical interaction will influence the deformation pattern of the myocardium. According to our hypothesis on myofiber reorientation, myofiber orientations will be influenced as well. Indeed, experimental results show differences in myofiber orientation between septum and LV free wall [12]. Restructuring alone does not describe the complete adaptive performance of the heart. Other adaptation mechanisms, such as changes in wall mass and cavity volume in response to pressure and volume overload respectively, are likely to be active as well.

\subsection{Conclusion}

In conclusion, circumferential-radial shear $E_{c r}$ and myofiber strain $E_{f}$, predicted from a combined model of LV mechanics and remodeling of fiber orientation, were found to become more physiologic by extending the mechanics model with physiological sequence of activation and triaxial active stress development. These extensions did hardly affect the pattern of the transverse component in myofiber orientation emerging during restructuring of the LV wall. The development of this transverse component in myofiber orientation is favorable for global pump and local myofiber function. 


\section{References}

1. Arts T, Prinzen FW, Snoeckx LH, Rijcken JM, and Reneman RS (1994). “Adaptation of cardiac structure by mechanical feedback in the environment of the cell: a model study". Biophysical Journal 66, pp. 953-961.

2. Ashikaga H, Omens JH, Ingels Jr NB, and Covell JW (2004). “Transmural mechanics at left ventricular epicardial pacing site". Am J Physiol - Heart Circ Physiol 286, H2401-H2407.

3. Ashikaga H, Coppola BA, Hopenfeld B, Leifer ES, McVeigh ER, and Omens JH (2007). "Transmural dispersion of myofiber mechanics: implications for electrical heterogeneity in vivo." J Am Coll Cardiol 49.8, pp. 909-916.

4. Ashikaga H, Spoel TIG van der, Coppola BA, and Omens JH (2009). "Transmural myocardial mechanics during isovolumic contraction." JACC Cardiovasc Imaging 2.2, pp. 202-211.

5. Bovendeerd PHM, Kroon W, and Delhaas T (2009). "Determinants of left ventricular shear strain". Am J Physiol - Heart Circ Physiol 297.3, H1058-H1068.

6. Bovendeerd P, Arts T, Huyghe J, Campen D van, and Reneman R (1992). “Dependency of local left ventricular wall mechanics on myocardial myofiber orientation: a model study". $J$ Biomech 25, pp. 1129-1135.

7. Caulfield J and Borg T (1979). "The collagen network of the heart". Lab. Invest. 40, pp. 364-372.

8. Delhaas T, Kroon W, Decaluwe W, Rubbens M, Bovendeerd P, and Arts T (2008). "Structure and torsion of the normal and situs inversus totalis cardiac left ventricle; Part I: experimental data in humans". Am J Physiol - Heart Circ Physiol 295, H197-H201.

9. Dokos S, Smaill BH, Young AA, and LeGrice IJ (2002). "Shear properties of passive ventricular myocardium". Am J Physiol 283, H2650-H2659.

10. Durrer D, van Dam RT, Freud GE, Janse MJ, Meijler FL, and Arzbaecher RC (1970). "Total excitation of the isolated human heart." Circulation 41.6, pp. 899-912.

11. Geerts-Ossevoort L, Bovendeerd P, Nicolay K, and Arts T (2002). "Characterization of the normal cardiac myofiber field in goat measured with MR-diffusion tensor imaging". Am J Physiol - Heart Circ Physiol 283.1, H139-H145.

12. Gilbert S, Benson A, Li P, and Van Holden A (2007). "Regional localisation of left ventricular sheet structure: integration with current models of cardiac fibre, sheet and band structure". Eur J Cardiothorac Surg 32, pp. 231-249.

13. Greenbaum R, Ho S, Gibson D, Becker A, and Anderson R (1981). "Left ventricular fibre architecture in man". Br Heart J 45, pp. 248-263.

14. Guccione J, Costa K, and McCulloch A (1995). "Finite element stress analysis of left ventricular mechanics in the beating dog heart." J Biomech 28.10, pp. 1167-1177.

15. Kerckhoffs RCP, Bovendeerd PHM, Kotte JCS, Prinzen FW, Smits K, and Arts T (2003). "Homogeneity of cardiac contraction despite physiological asynchrony of depolarization: a model study." Ann Biomed Eng 31.5, pp. 536-547.

16. Kroon W, Delhaas T, Arts T, and Bovendeerd P (2009). “Computational analysis of the myocardial structure: Adaptation of myofiber orientations through deformation in three di- 
mensions". Med Imag Anal 13, pp. 346-353.

17. Lin D and Yin FC (1998). "A multiaxial constitutive law for mammalian left ventricular myocardium in steady-state barium contracture or tetanus". J Biomech Eng 120(4), 504:517.

18. Moore CC, H LOC, McVeigh ER, and Zerhouni EA (2000). "Three-dimensional systolic strain patterns in the normal human left ventricle: Characterization with tagging MR Imaging". Radiology 214, pp. 453-466.

19. Nash MP and Hunter PJ (2000). "Computational mechanics of the heart". J Elasticity 61, pp. 113-141.

20. Nielsen PM, LeGrice IJ, Smaill BH, and Hunter PJ (1991). “Mathematical model of geometry and fibrous structure of the heart". Am J Physiol - Heart Circ Physiol 260, H1365-H1378.

21. Omens JH, May KD, and McCulloch AD (1991). "Transmural distribution of threedimensional strain in the isolated arrested canine left ventricle." Am J Physiol 261.3 Pt 2, H918H928.

22. Pluijmert M, Kroon W, Delhaas T, and Bovendeerd P (2012a). "Adaptive reorientation of cardiac myofibers: the long-term effect of initial and boundary conditions". Mechanics Research Communications 42, pp. 60-67.

23. Pluijmert M, Kroon W, Rossi AC, Bovendeerd PHM, and Delhaas T (2012b). "Why SIT works: normal function despite typical myofiber pattern in Situs Inversus Totalis (SIT) hearts derived by shear-induced myofiber reorientation." PLoS Comput Biol 8.7, e1002611.

24. Sermesant M, Rhode K, Sanchez-Ortiz GI, Camara O, Andriantsimiavona R, Hegde S, Rueckert D, Lambiase P, Bucknall C, Rosenthal E, Delingette H, Hill DLG, Ayache N, and Razavi R (2005). "Simulation of cardiac pathologies using an electromechanical biventricular model and XMR interventional imaging." Med Image Anal 9.5, pp. 467-480.

25. Streeter Jr D, Spotnitz HM, Patel DP, Ross Jr J, and Sonnenblick EH (1969). “Fiber orientation in the canine left ventricle during diastole and systole." Circ Res 24.3, pp. 339-347.

26. Ubbink SWJ, Bovendeerd PHM, Delhaas T, Arts T, and Van de Vosse FN (2006). "Towards model-based analysis of cardiac MR tagging data: Relation between left ventricular shear strain and myofiber orientation". Med Imag Anal 10, pp. 623-641.

27. Usyk TP, Mazhari R, and McCulloch AD (2000). "Effect of laminar orthotropic myofiber architecture on regional stress and strain in the canine left ventricle". J Elasticity 61, pp. 143164.

28. Waldman LK, Nosan D, Villarreal F, and Covell JW (1988). "Relation between transmural deformation and local myofiber direction in canine left ventricle". Circ Res 63, pp. 550-562.

29. Young A, Kramer C, Ferrari V, Axel L, and Reichek N (1994). “Three-dimensional left ventricular deformation in hypertrophic cardiomyopathy". Circulation 90, pp. 854-867. 


\section{Chapter 5}

\section{Determinants of biventricular cardiac function}

In preparation:

M. Pluijmert, T. Delhaas, A. Flores de la Parra, W. Kroon, F.W. Prinzen, P.H.M. Bovendeerd, Determinants of Biventricular Cardiac Function: a mathematical model study on geometry and myofiber orientation 


\section{Abstract}

Mathematical models of cardiac electromechanics are evolving to a state where they can assist in clinical decision making. Usually, these models combine a patient-specific geometry, a generic myofiber orientation, and a map of myocardial tissue properties tuned to the patient. However, it remains unclear to what extent deviations in myofiber orientation and geometry between model and patient influence predictions on cardiac function from these models. Therefore, the sensitivity of cardiac function for geometry and myofiber orientation was evaluated in a biventricular (BiV) finite element (FE) model of cardiac mechanics. Starting out from a reference geometry in which myofiber orientation had no transmural component, two new geometries were defined with either a $27 \%$ decrease in LV short-to-long axis ratio, or a $16 \%$ decrease of RV length, but identical LV and RV cavity and wall volumes. These variations in geometry caused differences in both local myofiber and global pump work below $7 \%$. Next, we allowed adaptive myofiber reorientation in each geometry. This resulted in an average change in orientation of $\sim 8^{\circ}$, predominantly through the formation of a myofiber component in transmural direction. In all three geometries, the change in orientation was accompanied by a considerable increase in local myofiber work $(\sim 16 \%)$ and in global pump work $(\sim 19 \%)$, differences in work being again small $(<14 \%)$ in between geometries. The findings suggest that implementing a realistic myofiber orientation is at least as important as defining a patient-specific geometry. The currently used model for remodeling of myofiber orientation seems a useful approach to estimate myofiber orientation in absence of accurate patient-specific information. 


\subsection{Introduction}

In the last decade, several biventricular (BiV) finite element (FE) models have been developed as a step towards model-assisted clinical decision making in cardiac disease $[1,6,12,18,24,32,47,51]$. The strength of these models is their ability to describe the relation between local tissue function and global pump function.

BiV FE models take into account the heart's geometry, myofiber orientation, and myocardial tissue properties. Preferably, the model input should be directly based on measurements. Indeed, geometrical characteristics can be captured from imaging data and included into models through sophisticated meshing algorithms [20]. However, determination of local myofiber orientation and material properties is more challenging. While first steps have been made to measure myofiber orientations in vivo using diffusion tensor magnetic resonance imaging (DTMRI) [45], researchers still have to rely on generic data from in vitro DTMRI [36] or histological measurements [21, 26] and morph these data into geometrically correct models [27, 48]. Next, myocardial tissue properties are tuned for maximum agreement between model predicted and clinically measured cardiac function [1, 24]. However, the available clinical information is not sufficient to determine cardiac tissue properties unequivocally.

The assumption of using a generic myofiber orientation becomes more valid when measurements of this generic myofiber orientation can be performed to such an accuracy that the variation of model predicted cardiac function within this measurement error is small. The accuracy of DTMRI is on the order of $\pm 10^{\circ}$ [30], even in the in vitro case [15, 34]. This accuracy seems to be sufficient for modeling cardiac electrophysiology [48]. However, in models of only left ventricular (LV) mechanics it has been shown that variations in the spatial distribution of myofiber orientations of $8^{\circ}$, introduced variations in myofiber stress of $10 \%$ [10]. In addition, shear deformation was only predicted realistically when a transmural component in myofiber orientation of $\pm 10^{\circ}$ was included [5]. As an alternative approach for estimating myofiber orientation, we hypothesized that myofiber orientation is the result of a successful adaptation process [3]. This hypothesis was tested in an LV model that described the adaptive change of myofiber orientation in response to fiber cross-fiber shear and results were promising $[19,28]$.

While sensitivity of LV mechanics for myofiber orientation was found to be high, sensitivity to geometry was low [10]. Similar variations in myofiber stress of $10 \%$ could only be obtained when a more drastic geometrical variation in the short-to-long axis ratio from 1:1 to 1:5 was applied. However, these results might not be valid a $\mathrm{BiV}$ model, among others because of the nonsymmetrical shape of the $\mathrm{BiV}$ and the attachment of right ventricle (RV) and 
Figure 5.1: $\quad \mathrm{BiV}$ geometries that result from parameter values as presented in table 5.1. A: Illustration of geometrical parameters in crosssections of the REF geometry. Short-axis cross-section visualizing radii $R_{i}$, which define parameters $f_{\text {sep }}, f_{R}$, and $f_{T}$ (left). Long-axis cross-section of the BiV geometry visualizing parameters $\theta_{A}$ and $h / Z_{1}$ $\left(f_{h}\right)$ (right). B: FE mesh of simulation REF on the left, and myofiber orientation vector in the unloaded state $\vec{e}_{f, 0}$ on the right. $\quad \vec{e}_{f, 0}$ is quantified by helix angle $\alpha_{h, 0}$ and transverse angle $\alpha_{t, 0}$ using a local cardiac coordinate system $\left\{\vec{e}_{t, 0}, \vec{e}_{l, 0}, \vec{e}_{c, 0}\right\}$. C: Two other FE meshes were created: a more elongated mesh (LONG) and a mesh with a higher attachment of the right to the left ventricle (RVAT). All the three meshes consist of 684 elements.
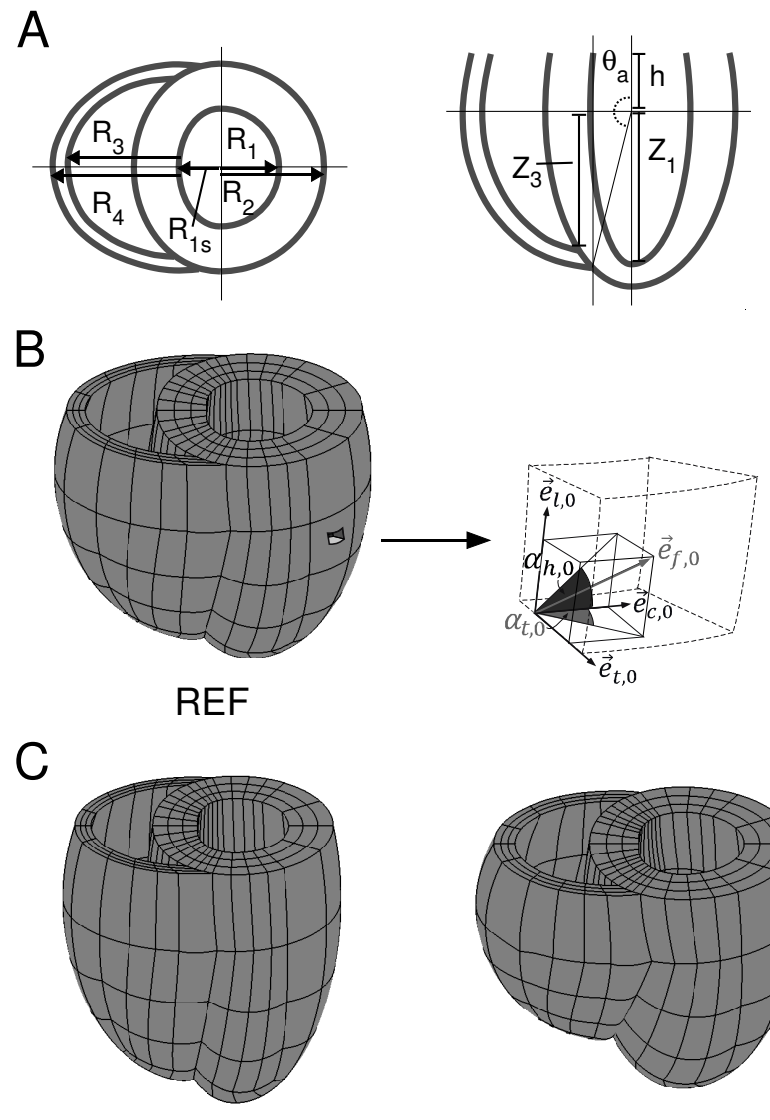

LONG

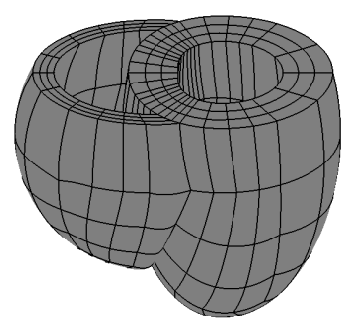

RVAT

LV. It remains unclear to what extent changes in geometry and in myofiber orientation also influence predictions on cardiac function from BiV finite element (FE) models.

The aim of this study was to investigate the sensitivity of cardiac function to geometry and myofiber orientation in a BiV FE model of cardiac mechanics. First, local myofiber function and global pump function were compared between three geometries with an either $27 \%$ difference in LV short-to-long axis ratio or a $16 \%$ difference in RV length, but otherwise no differences between the three geometries with respect to LV and RV cavity and wall volumes, and myofiber orientation. Next, in each of these three models the sensitivity for myofiber orientation was tested by letting the myofiber orientation change according to the reorientation model in [19]. We evaluated the effect of adaptive myofiber reorientation in terms of local and global function and in terms of cardiac deformation. 
Table 5.1: Biventricular geometry input parameter values. See figure 5.1A for definition of $R^{\prime}$ s, $Z^{\prime}$ s, $h$, and $\theta_{A}$

\begin{tabular}{|c|c|c|c|c|}
\hline parameter & definition & value & unit & explanation \\
\hline$\overline{\overline{V_{l v w}}}$ & & 160 & $\overline{[\mathrm{ml}]}$ & "LV wall volume \\
\hline$f_{V}$ & $V_{l v, 0} / V_{l v w}$ & 0.375 & {$[-]$} & $\begin{array}{l}\mathrm{LV} \text { cavity-to-wall volume } \\
\text { ratio in the stress-free } \\
\text { state }\end{array}$ \\
\hline$f_{V R L}$ & $V_{r v, 0} / V_{l v, 0}$ & 1.0 & {$[-]$} & $\begin{array}{l}\text { RV-to-LV cavity volume } \\
\text { ratio }\end{array}$ \\
\hline$f_{R}$ & $R_{1} /\left(h+Z_{1}\right)$ & $0.55(0.40)^{*}$ & {$[-]$} & $\begin{array}{l}\text { Endocardial LV radius-to- } \\
\text { length ratio }\end{array}$ \\
\hline$f_{h}$ & $h / Z_{1}$ & 0.40 & {$[-]$} & $\begin{array}{l}\text { Truncation height above } \\
\text { equator-to-LV endocar- } \\
\text { dial length ratio }\end{array}$ \\
\hline$f_{\text {sep }}$ & $R_{1 s} / R_{1}$ & 0.70 & {$[-]$} & $\begin{array}{l}\text { Septal endocardial radius } \\
\left(R_{1 s}\right) \text {-to-LV endocardial } \\
\text { radius }\left(R_{1}\right) \text { ratio }\end{array}$ \\
\hline$f_{T}$ & $\frac{R_{2}-R_{1}}{R_{4}-R_{3}}$ & $0.33(0.35)^{\dagger}$ & {$[-]$} & $\begin{array}{l}\text { LV-to-RV wall thickness } \\
\text { ratio }\end{array}$ \\
\hline$\theta_{A}$ & & $0.85 \pi(0.75 \pi)^{\dagger}$ & [rad] & $\begin{array}{l}\text { Lowest attachment angle } \\
\text { of RV to LV }\end{array}$ \\
\hline
\end{tabular}

${ }^{*}$ The value between brackets holds for simulation $L O N G .{ }^{+}$The values between brackets hold for simulation RVAT.

\subsection{Methods}

\section{Geometry}

In the passive stress-free state, the biventricular $(\mathrm{BiV})$ geometry was described by two intersecting, truncated ellipsoids (figure 5.1), defined by 8 input parameters (table 5.1). Left ventricular wall volume $V_{\text {lvw }}$ determines the overall size, whereas dimensionless parameters $f_{V}, f_{s e p}, f_{R}, f_{h}, f_{T}$, and $f_{V R L}$ determine shape. Explanation of the parameters can be found in figure 5.1 and table 5.1. $\theta_{A}$ marks the attachment of the right ventricle (RV) epicardial apex to the LV epicardial wall and therewith it determines the location of the center of the RV ellipsoid.

\section{Myofiber orientation}

Myofiber orientation in the unloaded state $\vec{e}_{f, 0}$ was defined in a local cardiac coordinate system $\left\{\vec{e}_{t, 0}, \vec{e}_{l, 0}, \vec{e}_{c, 0}\right\}$ (figure 5.1B). The transmural direction $\vec{e}_{t, 0}$ was defined perpendicular to the endo- and epicardial surfaces. The longitu- 
dinal direction $\vec{e}_{l, 0}$ was defined parallel to these surfaces from apex to base. To obtain a right-handed coordinate system, the circumferential direction $\vec{e}_{c, 0}$ was defined in clockwise direction when viewing the geometry in apex-tobase direction. In the local cardiac coordinate system, $\vec{e}_{f, 0}$ was quantified by two angles. The helix angle $\alpha_{h, 0}$ was defined as the angle between $\vec{e}_{c, 0}$ and the projection of $\vec{e}_{f, 0}$ on the circumferential-longitudinal plane $\left(\vec{e}_{c, 0}, \vec{e}_{l, 0}\right)$. The transverse angle $\alpha_{t, 0}$ was defined as the angle between $\vec{e}_{c, 0}$ and the projection of $\vec{e}_{f, 0}$ on the circumferential-transmural plane $\left(\vec{e}_{c, 0}, \vec{e}_{t, 0}\right)$. In this BiV model, we used the bi-directional spherical linear interpolation method of Bayer et al. [4] to ensure a smooth transition of the coordinate system and myofiber orientation in all regions, including the RV-LV attachment.

During simulation of the cardiac cycle, deformation of the tissue caused a change from the reference orientation $\vec{e}_{f, 0}$ into an actual myofiber orientation $\vec{e}_{f}$ (figure 5.2A). In addition, $\vec{e}_{f, 0}$ was subject to adaptation that caused a structural change in myofiber orientation in the unloaded reference state. Adaptation of $\vec{e}_{f, 0}$ was simulated with the model published by Kroon et al. [19]. The model is explained in detail in figure 5.2 and is described by the following evolution equation:

$$
\frac{\partial \vec{e}_{f, 0}}{\partial t}=\frac{1}{\kappa}\left(\vec{e}_{f}^{*}-\vec{e}_{f, 0}\right)
$$

with an adaptation time constant $\mathcal{k}$ that was set to four times the cardiac cycle time. $\vec{e}_{f}^{*}$ is the actual myofiber orientation in the deformed tissue corrected for rigid body rotation:

$$
\vec{e}_{f}=\frac{\boldsymbol{F} \cdot \vec{e}_{f, 0}}{\lambda_{f}}=\frac{\boldsymbol{R} \cdot \boldsymbol{U} \cdot \vec{e}_{f, 0}}{\lambda_{f}}=\boldsymbol{R} \cdot \vec{e}_{f}^{*} ; \quad \lambda_{f}=\left|\boldsymbol{U} \cdot \vec{e}_{f, 0}\right|
$$

with $\lambda_{f}$ the myofiber stretch ratio and $\boldsymbol{F}$ the deformation gradient tensor that consists of the actual deformation $U$ and rigid body rotation $\boldsymbol{R}$. Since rigid body rotations are not sensed by the tissue, they are not taken into account for adaptation. Notice that myofiber reorientation only occurs in case none of the principal strain directions (eigenvectors of $\boldsymbol{U}$ ) coincides with the unloaded fiber direction $\vec{e}_{f, 0}$, i.e., in case of fiber cross-fiber shear. To ensure that myofibers do not stick out of the endo- and epicardial surfaces, the adapted myofiber orientation was forced to be parallel with these surfaces.

\section{Material properties}

Myocardial tissue Cauchy stress $\sigma$ was composed of a passive component $\sigma_{p}$ and an active component $\sigma_{a}$ :

$$
\sigma=\sigma_{p}+\sigma_{a} \vec{e}_{f} \vec{e}_{f}
$$




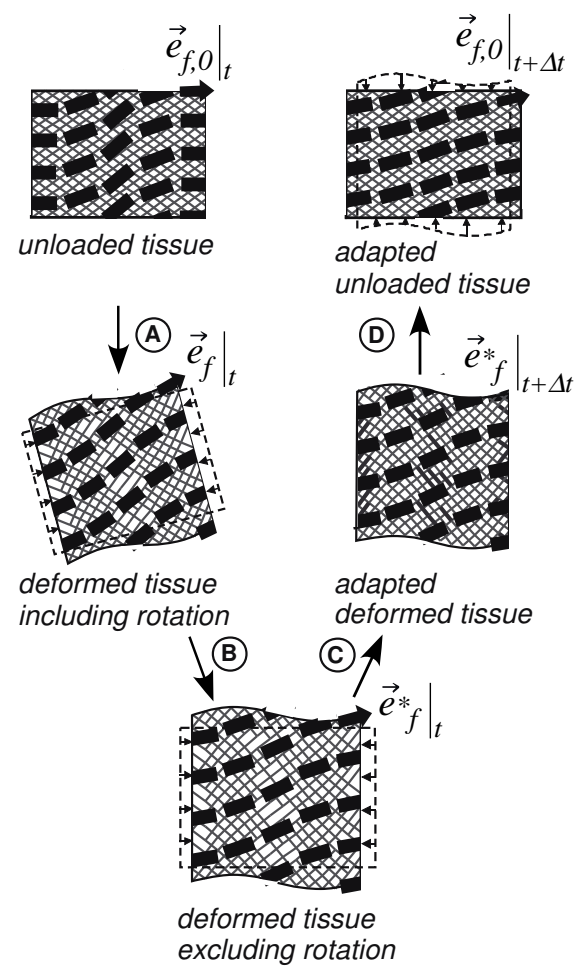

Figure 5.2: Conceptual model for myofiber reorientation adapted from [19] and expressed in equation 5.1. The myofiber orientation vector is denoted by $\vec{e}_{f, 0}$ in the unloaded configuration at time step $t$. During a cardiac cycle, the myocardial tissue deforms (A). The myofiber orientation vector in the deformed configuration is denoted by $\vec{e}_{f}$. It is assumed that shear forces due to deformation of the tissue will damage the connections between extra-cellular matrix (ECM, raster) and myofibers (thick black lines). Rigid body rotations are not sensed by the tissue and therefore excluded from adaptation (B). Rotation-corrected myofiber orientation is denoted by $\vec{e}_{f}^{*}$. New connections between the ECM and the myofibers will be formed continuously (C). When a connection is made, myofibers tend to be fixed within the tissue (D). The adapted myofiber orientation is used as the orientation in the unloaded state at time step $t+\Delta t$. 
with $\vec{e}_{f}$ the current myofiber direction in the deformed tissue. Passive material behavior was assumed nonlinearly elastic, transversely isotropic, and nearly incompressible. It was described by a strain energy density function that consisted of an isotropic, an anisotropic, and a volume part as described in Kerckhoffs et al. [17]. Active stress $\sigma_{a}$ was modeled through a series arrangement of a contractile and a series elastic element. The magnitude of $\sigma_{a}$ depended on time elapsed since activation $t_{a}$, sarcomere length $l_{s}$, and sarcomere shortening velocity. A complete description of the active stress model can be found in [5]. Active stress was initiated simultaneously throughout both ventricles with a cycle time of $800 \mathrm{~ms}$.

\section{Governing equations and boundary conditions}

In the model, the quasi-static equations of conservation of linear momentum were solved:

$$
\vec{\nabla} \cdot \sigma=\overrightarrow{0}
$$

with $\vec{\nabla}$ the spatial gradient operator. At the base, essential boundary conditions were defined to suppress rigid body motion and to represent the mechanical effect of structures left out of the model, e.g., the valvular annulus. The epicardial surface was assumed to be traction free while the RV and LV endocardial surfaces were uniformly subjected to a right and left ventricular pressure, $p_{r v}$ and $p_{l v}$, respectively. During isovolumic contraction (IC) and relaxation (IR) phases of the cardiac cycle, $p_{r v}$ and $p_{l v}$ are determined such that mechanical equilibrium of the myocardial tissue was obtained at a constant end-diastolic or end-systolic RV and LV volume, respectively. During the filling and ejection phase, $p_{r v}$ and $p_{l v}$ were computed from the interaction of the RV and LV with lumped parameter models of the pulmonary and systemic circulation, respectively (figure 5.3). Parameter values of the resistances and compliances were chosen such that they are representative for characteristics of the human circulation $[38,37]$ and are listed in table 5.2.

\section{Numerical implementation}

The equilibrium equations (5.4) were solved numerically with a Galerkin type finite element method using 27-noded hexahedral elements with a tri-quadratic interpolation of the displacement field. The mesh consisted of 684 elements and 19803 degrees of freedom. The timestep was fixed at $1 \mathrm{~ms}$. It took about 2 hours to compute one cardiac cycle on a single core of an Intel Core i7-2600 processor on a personal computer. 


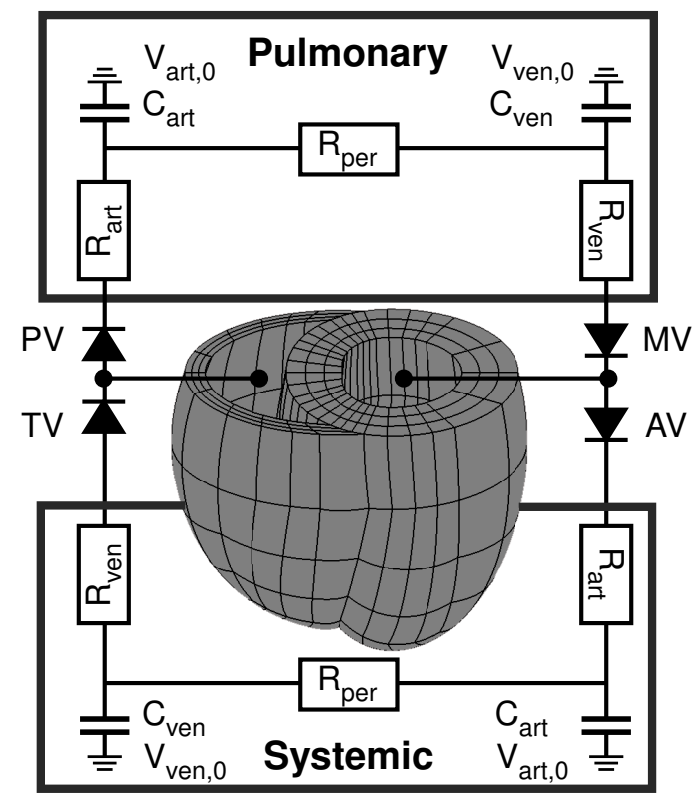

Figure 5.3: The $\mathrm{BiV}$ finite element (FE) mesh is incorporated in a lumped parameter model of the circulation. The valves are modeled as ideal diodes: $\mathrm{AV}$, aortic valve; $\mathrm{MV}$, mitral valve; PV, pulmonary valve; $\mathrm{TV}$, tricuspid valve. Both pulmonary and systemic circulation are modeled using compliances and resistances: $C_{\text {art }}$, arterial compliance; $C_{\text {ven }}$, venous compliance; $R_{\text {art }}$, arterial resistance; $R_{\text {per }}$, peripheral resistance; $R_{\text {ven }}$, venous resistance; $V_{\text {art }, 0}$, zero-pressure arterial volume; $V_{\text {ven, }, 0}$, zero-pressure venous volume. Parameter values are listed in table 5.2.

Table 5.2: Parameter values for the pulmonary and systemic circulation.

\begin{tabular}{lllll}
\hline parameter & definition & unit & systemic & pulmonary \\
\hline \hline$R_{\text {art }}$ & outflow resistance & {$\left[\mathrm{kPa} \cdot \mathrm{s} \cdot \mathrm{ml}^{-1}\right]$} & $4.46 \cdot 10^{-3}$ & $2.48 \cdot 10^{-3}$ \\
$R_{\text {per }}$ & peripheral resistance & {$\left[\mathrm{kPa} \cdot \mathrm{s} \cdot \mathrm{ml}^{-1}\right]$} & $1.49 \cdot 10^{-1}$ & $1.78 \cdot 10^{-2}$ \\
$R_{\text {ven }}$ & inflow resistance & {$\left[\mathrm{kPa} \cdot \mathrm{s} \cdot \mathrm{ml}^{-1}\right]$} & $1.10 \cdot 10^{-3}$ & $2.18 \cdot 10^{-3}$ \\
$C_{\text {art }}$ & arterial compliance & {$\left[\mathrm{ml} \cdot \mathrm{kPa}{ }^{-1}\right]$} & $1.53 \cdot 10^{1}$ & $4.59 \cdot 10^{1}$ \\
$C_{\text {ven }}$ & venous compliance & {$\left[\mathrm{ml} \cdot \mathrm{kPa}{ }^{-1}\right]$} & $4.59 \cdot 10^{1}$ & $1.53 \cdot 10^{1}$ \\
$V_{\text {art }, 0}$ & arterial unstressed volume & {$[\mathrm{ml}]$} & $7.04 \cdot 10^{2}$ & $7.83 \cdot 10^{1}$ \\
$V_{\text {ven }, 0}$ & venous unstressed volume & {$[\mathrm{ml}]$} & $3.00 \cdot 10^{3}$ & $3.13 \cdot 10^{2}$ \\
$V_{\text {tot }}$ & total blood volume & {$[\mathrm{ml}]$} & $5.00 \cdot 10^{3}$ & \\
\hline
\end{tabular}




\section{Simulations performed}

To investigate the sensitivity of cardiac function for geometry, three different geometries were created that all had an LV wall volume of $160 \mathrm{ml}$ and an RV wall volume of $40 \mathrm{ml}$. In the unloaded state, both LV and RV cavity volume was $60 \mathrm{ml}$. First, a reference geometry REF (figure 5.1B) was defined with parameter values listed in table 5.1. By changing parameter $f_{R}$ from 0.55 to 0.40 a more elongated geometry LONG was created (figure 5.1C, left). In the third geometry RVAT, the attachment of the RV was located more towards the equator by changing $\theta_{A}$ from $0.85 \pi$ to $0.75 \pi$ (figure $5.1 \mathrm{C}$, right). To maintain the same RV wall volume, the LV-to-RV wall thickness ratio $f_{T}$ was changed from 0.33 to 0.35. Dimensions of the geometries are listed in table 5.3.

In all geometries, the initial distribution of $\alpha_{h, 0}$ varied nonlinearly with the transmural position from $65^{\circ}$ at the endocardium to $-50^{\circ}$ at the epicardium [5]. The initial condition for $\alpha_{t, 0}$ was set to zero. Still, a transmural component in myofiber orientation is already observed near the attachment regions (see dashed lines in septal long-axis cross-sections in figure 5.5) as a result of the interpolation method [4].

The sensitivity of cardiac function for myofiber orientation was investigated by adaptive reorientation of myofibers. The first 8 consecutive cardiac cycles were used to approximate a hemodynamic steady state while myofiber reorientation was disabled. In the subsequent 30 cardiac cycles myofiber orientation was adapted per node according to the adaptation model (equation 5.1). The emerging structure will be referred to as adapted state. The structure we started with is referred to as initial state.

Results from all three geometries were analyzed in both the initial and adapted state in terms of myofiber orientation $\alpha_{h, 0}$, and $\alpha_{t, 0}$, the angle $\gamma$ between the initial and adapted orientation, global pump function as well as local myofiber function. Global function was quantified as cardiac output (CO), $\mathrm{LV}$ ejection fraction (EF), and stroke work $W$, which was calculated from the area within the pressure-volume loops for both ventricles:

$$
W=\oint p_{c a v} d V_{c a v}
$$

with $p_{c a v}$ and $V_{c a v}$ the cavity pressure and volume, respectively. Local function was quantified as natural myofiber strain during isovolumic contraction $\epsilon_{f, i c}$, during ejection $\epsilon_{f, e j}$, during isovolumic relaxation $\epsilon_{f, i r}$, maximum active myofiber Cauchy stress $\sigma_{f, \max }$, and stroke work density $w_{f}$ calculated from the area within the active myofiber Cauchy stress-natural strain loop:

$$
w_{f}=\oint \sigma_{f} d \epsilon_{f} ; \quad \epsilon_{f}=\ln \lambda_{f}
$$


Table 5.3: Biventricular geometry output parameter values. See figure 5.1A for definition of $R^{\prime}$ s, $Z$ 's, and $h$.

\begin{tabular}{lllll}
\hline & \multicolumn{2}{l}{ Inner radius at equator [mm] } & \multicolumn{2}{c}{ Inner length $[\mathrm{mm}]$} \\
Geometry & $\mathrm{LV}\left(R_{1}\right)$ & $\mathrm{RV}\left(R_{3}\right)$ & $\mathrm{LV}\left(\mathrm{Z}_{1}+h\right)$ & $\mathrm{RV}\left(\mathrm{Z}_{3}+h\right)$ \\
\hline \hline REF & 20.2 & 52.8 & 73.6 & 69.5 \\
LONG & 18.2 & 48.1 & 90.7 & 83.9 \\
RVAT & 20.2 & 56.0 & 73.6 & 58.2 \\
\hline
\end{tabular}

with natural myofiber strain $\epsilon_{f}$ and active myofiber Cauchy stress $\sigma_{f}$. Local function variables are presented as mean and standard deviation (SD) calculated from the nodes indicated in the mesh in figure 5.5. To make sure that function values of the adapted state were determined in the hemodynamic steady state, we reran a simulation for each geometry with adapted myofiber orientation and disabled adaptive reorientation.

To further evaluate the model results, we analyzed various modes of deformation in the REF simulation. First we evaluated global changes during a cardiac cycle of LV inner length, LV inner diameter at equatorial level, and radial wall thickness at equatorial level. In addition, we compared model computed with experimentally determined circumferential-radial shear $E_{c r}$ [28]. See [5] and [8] for further details on the experimental data. Model predicted $E_{c r}$ was computed from a long-axis cross-section in the lateral LV free wall $(\mathrm{LVfw})$ and averaged in transmural direction.

\subsection{Results}

Sensitivity of cardiac function to geometry was investigated by comparing global cardiac function, expressed as LV stroke work $\left(W_{l v}\right)$, RV stroke work $\left(W_{r v}\right)$, cardiac output $(\mathrm{CO}), \mathrm{LV}$ ejection fraction (EF), and RV EF (table 5.4). In the initial state, mean $( \pm \mathrm{SD})$ value from the three geometries $R E F, L O N G$, and RVAT was $0.72(0.04) \mathrm{J}$ for $W_{l v}, 0.21(0.01) \mathrm{J}$ for $W_{r v}, 4.2(0.1) \mathrm{l} / \mathrm{min}$ for CO, 0.42 (0.02) for LV EF, and 0.56 (0.01) for RV EF. Only small difference between geometries were present: SD is about $6 \%$ for $W_{l v}, 3 \%$ for $W_{r v}, 2 \%$ for $\mathrm{CO}, 4 \%$ for LV EF, and $2 \%$ for RV EF. Similarly, differences in mean myofiber function were small (table 5.5): mean $( \pm \mathrm{SD})$ myofiber strain during isovolumic contraction $\epsilon_{f, i c}$ was $-0.03(0.002)$ and varied $\sim 7 \%$, during ejection $\epsilon_{f, e j}$ was -0.13 (0.005) and varied $\sim 4 \%$, during isovolumic relaxation was $0.03(0.002)$ and varied $\sim 7 \%$, maximum myofiber stress $\sigma_{f, \max }$ was $41.8(0.5) \mathrm{kPa}$ and varied 
Table 5.4: Global function presented as LV stroke work $W_{l v}$, RV stroke work $W_{r v}$, cardiac output $\mathrm{CO}$, and $\mathrm{LV}$ and RV ejection fraction EF.

\begin{tabular}{lcccccc}
\hline & \multicolumn{2}{c}{ REF } & \multicolumn{2}{c}{$L O N G$} & \multicolumn{2}{c}{ RVAT } \\
& init & adap & init & adap & init & adap \\
\hline \hline$W_{l v}[\mathrm{~J}]$ & 0.71 & 0.86 & 0.76 & 0.85 & 0.68 & 0.85 \\
$W_{r v}[\mathrm{~J}]$ & 0.21 & 0.24 & 0.22 & 0.24 & 0.21 & 0.24 \\
$\mathrm{CO}[\mathrm{ml} / \mathrm{min}]$ & 4.2 & 4.8 & 4.3 & 4.6 & 4.1 & 4.7 \\
$\mathrm{LV} \mathrm{EF} \mathrm{[-]}$ & 0.42 & 0.53 & 0.44 & 0.52 & 0.41 & 0.52 \\
$\mathrm{RV} \mathrm{EF} \mathrm{[-]}$ & 0.55 & 0.57 & 0.57 & 0.58 & 0.55 & 0.58 \\
\hline
\end{tabular}

Table 5.5: Local function presented as (mean $\pm S D$ ) natural myofiber during isovolumic contraction $\epsilon_{f, i c}$, during ejection $\epsilon_{f, e j}$, during isovolumic relaxation $\epsilon_{f, i r}$, maximum active myofiber Cauchy stress $\sigma_{f, \max }$, and stroke work density $w_{f}$. Mean and SD were calculated from the nodes indicated in figure 5.5.

\begin{tabular}{ccccccc}
\hline & \multicolumn{2}{c}{ REF } & \multicolumn{2}{c}{ LONG } & \multicolumn{2}{c}{ RVAT } \\
& init & adap & init & adap & init & adap \\
\hline \hline$\epsilon_{f, i c}$ & $-0.04(0.02)$ & $-0.01(0.02)$ & $-0.03(0.02)$ & $-0.00(0.02)$ & $-0.03(0.02)$ & $-0.02(0.02)$ \\
$\epsilon_{f, e j}$ & $-0.12(0.02)$ & $-0.14(0.02)$ & $-0.13(0.02)$ & $-0.16(0.02)$ & $-0.12(0.02)$ & $-0.14(0.02)$ \\
$\epsilon_{f, i r}$ & $0.04(0.02)$ & $0.02(0.02)$ & $0.03(0.02)$ & $0.01(0.02)$ & $0.04(0.02)$ & $0.03(0.02)$ \\
$\sigma_{f, \text { max }}$ & $42.3(13.1)$ & $43.8(11.8)$ & $41.4(12.1)$ & $43.7(10.9)$ & $41.6(13.9)$ & $43.8(12.8)$ \\
$w_{f}$ & $5.0(1.8)$ & $5.7(1.7)$ & $5.2(1.7)$ & $6.1(1.6)$ & $4.8(1.8)$ & $5.6(1.6)$ \\
\hline
\end{tabular}

$\sim 1 \%$, stroke work density was $5.0(0.2) \mathrm{mJ} / \mathrm{ml}$ and varied $\sim 4 \%$. Coefficients of variation were $\sim 14 \%$ for $\epsilon_{f, e j}, \sim 31 \%$ for $\sigma_{f, \max }$, and $\sim 35 \%$ for $w_{f}$ (table 5.6). Sensitivity of cardiac function to myofiber orientation was investigated by initiating adaptive myofiber reorientation after 12 initial cardiac cycles, during which hemodynamic equilibrium was reached. The optimal structure was found after 20 cardiac cycles with adaptive myofiber reorientation. Thereafter, no changes in global and local function were observed. In figure 5.4, the change in myofiber orientation in the unloaded configuration $\vec{e}_{f, 0}$ between the initial and adapted state is visualized as the angle $\gamma$. The mean $( \pm \mathrm{SD})$ change was $8.0^{\circ}(6.3)$ in REF, $7.8^{\circ}$ (5.8) in $L O N G$, and $7.9^{\circ}$ (6.6) in RVAT. In $13 \%$ of the nodes, $\gamma$ was $15^{\circ}$ or more and these nodes were typically located at the apical, basal and attachment regions (white regions in the figure).

The change in myofiber orientation was further quantified by the helix 
Table 5.6: Coefficients of variations defined as SD/mean as an indication for homogeneity of myofiber strain during ejection $\left(C_{v, \epsilon f}\right)$, of maximum myofiber stress $\left(C_{v, \sigma f}\right)$, and of myofiber work density $\left(C_{v, w f}\right)$.

\begin{tabular}{lllllll}
\hline & \multicolumn{2}{c}{ REF } & \multicolumn{2}{c}{ LONG } & \multicolumn{2}{c}{ RVAT } \\
& init & adap & init & adap & init & adap \\
\hline \hline$C_{v, \epsilon f}$ & 0.1099 & 0.0817 & 0.1687 & 0.1271 & 0.1447 & 0.1269 \\
$C_{v, \sigma f}$ & 0.3096 & 0.2704 & 0.2923 & 0.2494 & 0.3353 & 0.2922 \\
$C_{v, w f}$ & 0.3546 & 0.2953 & 0.3269 & 0.2623 & 0.3636 & 0.2920 \\
\hline
\end{tabular}

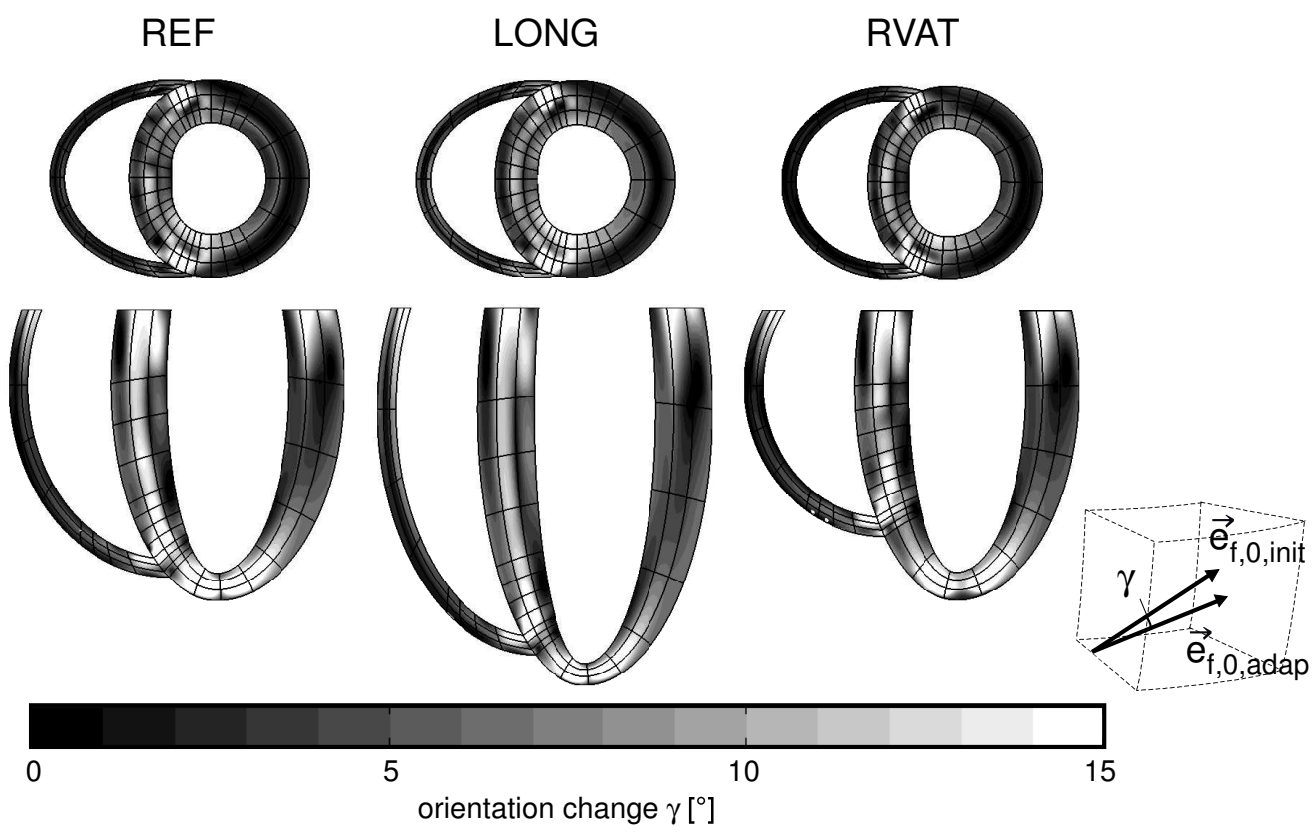

Figure 5.4: Orientation change defined as the angle $\gamma$ between the myofiber orientation in the initial state $\vec{e}_{f, 0, \text { init }}$ and in the final state $\vec{e}_{f, 0, a d a p}$. Top: short-axis crosssections at equatorial level. Bottom: Long-axes cross-sections in geometrical plane of symmetry. Note: For better visualization of spatial differences in $\gamma$ in the midmyocardium, the gray-scale was cut off at $15^{\circ}$. In about $13 \%$ of the nodes, a $\gamma$ of more the $15^{\circ}$ could be observed. These nodes were located in or near the attachment region. 
angle $\alpha_{h, 0}$ and the transverse angle $\alpha_{t, 0}$ (figures 5.5 and 5.6). More attention is paid to $\alpha_{t, 0}$ because the change in orientation took place especially in the transmural direction. Results on $\alpha_{t, 0}$ are only shown in detail from REF in figure 5.5, because adapted patterns for $\alpha_{t, 0}$ were similar in all three geometries REF, LONG, and RVAT. In the LVfw, a gradient in $\alpha_{t, 0}$ developed with negative values near the apex and positive values near the base (figure 5.5). Largest values for $\alpha_{t, 0}$ developed in the septum, especially at the RV endocardial side. In the basal region of the septum, $\alpha_{t, 0}$ was positive at the posterior side, but about zero at the anterior side. To a lesser extent, this asymmetry in myofiber orientation between anterior and posterior side was also seen in the LVfw and RVfw. In the RVfw, $\alpha_{t, 0}$ showed similar patterns as in the LVfw, but angles are smaller compared to the LVfw and septum.

Figure 5.6 shows the predicted distribution of $\alpha_{h, 0}$ and $\alpha_{t, 0}$. Model predicted data on $\alpha_{h, 0}$ was averaged from the anterior and posterior cross-sections of the LVfw, $\mathrm{LVfw}_{\text {ant }}$ and $\mathrm{LVfw}_{\text {post}}$, respectively (left panel). Here, it becomes clear that the distributions of $\alpha_{h, 0}$ remained similar in all three geometries, after reorientation. The right panel of figure 5.6 shows the small differences in $\alpha_{t, 0}$ that are present between the three geometries. Model predicted values of both $\alpha_{h, 0}$ and $\alpha_{t, 0}$ were very well within the range of experimental data for each of the simulations.

Both local and global function increased in all geometries from the initial to the adapted state. $W_{l v}$ increased by $19 \%, W_{r v}$ by $13 \%$, CO by $12 \%$, LV EF by $24 \%$, and RV EF by $4 \%$. Similarly, mean myofiber function increased (table 5.5): $\epsilon_{f, e j}$ decreased by $15 \%, \sigma_{f, \max }$ increased by $5 \%$, and $w_{f}$ increased by $16 \%$. Absolute myofiber strain during the isovolumic phases and coefficients of variation were decreased in the adapted state (table 5.5 and 5.6, respectively). In between geometries with adapted myofiber orientation differences in both local and global cardiac function were smaller than $14 \%$.

The pressure-volume relation of the three geometries $R E F, L O N G$, and RVAT in both the initial and the adapted state are shown in figure 5.7. The increase in $W_{l v}$ and $W_{r v}$ is clearly visible from the increased areas under the loops in the adapted state (black) as compared to the initial state (white). In addition, LV preload decreased, whereas RV preload increased after adaptive myofiber reorientation.

Figure 5.8 shows the temporal variation in global deformation in the initial (dashed) and adapted (solid) state of simulation REF. During ejection, LV longitudinal shortening increased from $8 \%$ (initial) to $10 \%$ (adapted), LV diameter shortening increased from $21 \%$ (initial) to $24 \%$ (adapted), and LV radial thickening increased from $26 \%$ (initial) to $31 \%$ (adapted). Similar trends were observed in LONG and RVAT.

Temporal variations in circumferential-radial shear $E_{c r}$ are shown in figure 5.9. We consider this a more critical parameter to evaluate the model 


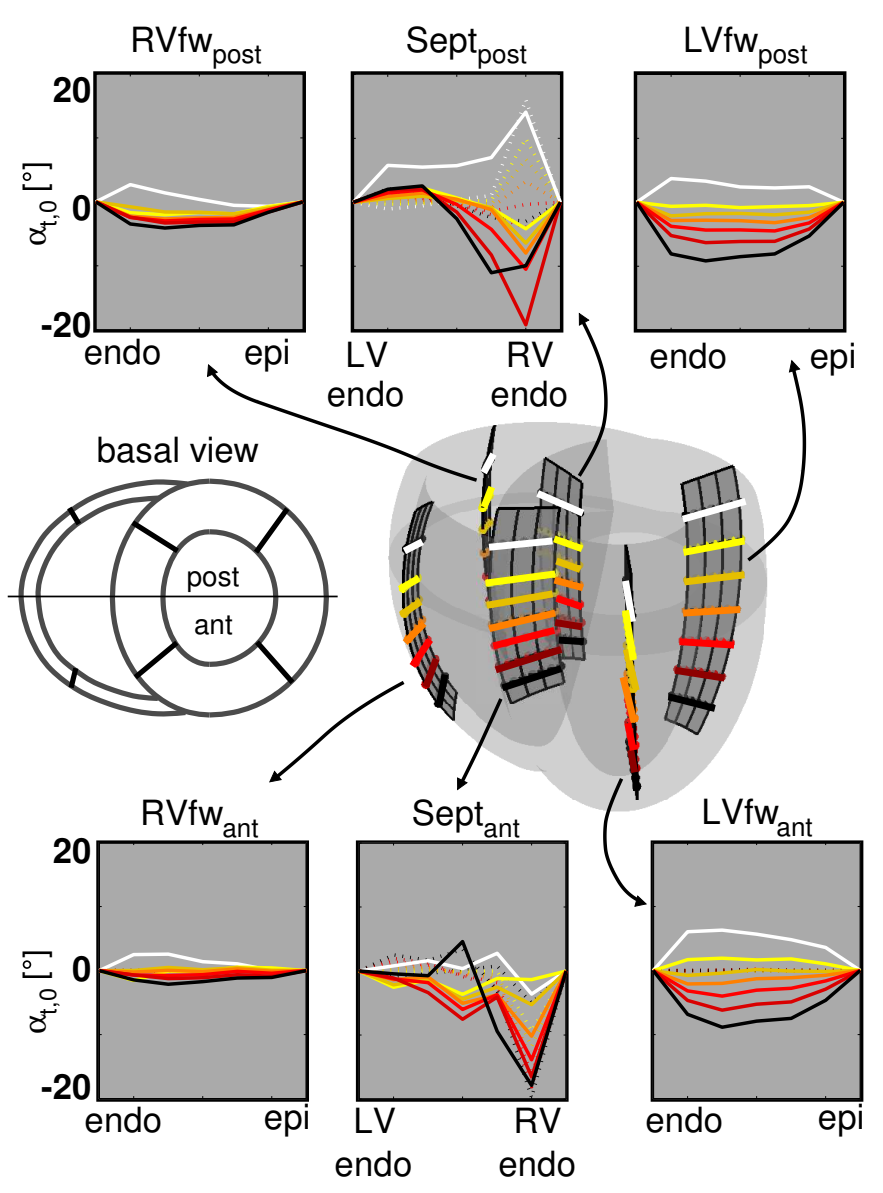

Figure 5.5: Initial (dashed) and adapted (solid) distributions of the transverse angle $\alpha_{t, 0}\left[{ }^{\circ}\right]$. Results are presented for the REF simulation. The middle panel displays the long axis cross sections for which the results are shown: two cross-sections in the LV free wall at the anterior $\left(\mathrm{LVfw}_{\text {ant }}\right)$ and posterior $\left(\mathrm{LVfw}_{\text {post }}\right)$ side, two in the septum (Sept $_{\text {ant }}$ and Sept $\left.t_{\text {post }}\right)$, and two in the RV free wall (RVfw ant and RVfw post). In each sections, results at 7 levels between apex and base are shown. The colors in the mesh corresponds with the colors of the lines in the graphs. 

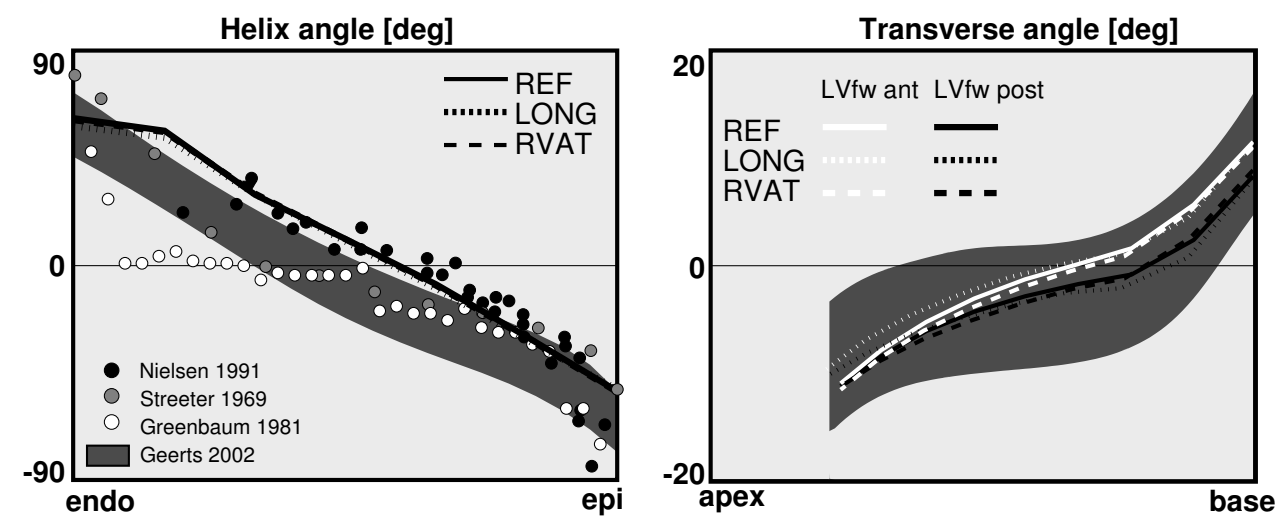

Figure 5.6: Adapted myofiber orientation angles resulting from the simulations (lines) and experimental data taken from [9, 11, 26, 42]. Left: The data on the helix angles $\alpha_{h, 0}$ was averaged from the anterior and posterior cross-sections of the LV free wall at equatorial level (figure 5.5). Differences between the simulations were small. Right: A difference in the transverse angle $\alpha_{t, 0}$ is observed between the posterior and anterior LVfw. Experimental data was only available from [9]. All results from the simulations fall within the measured range.

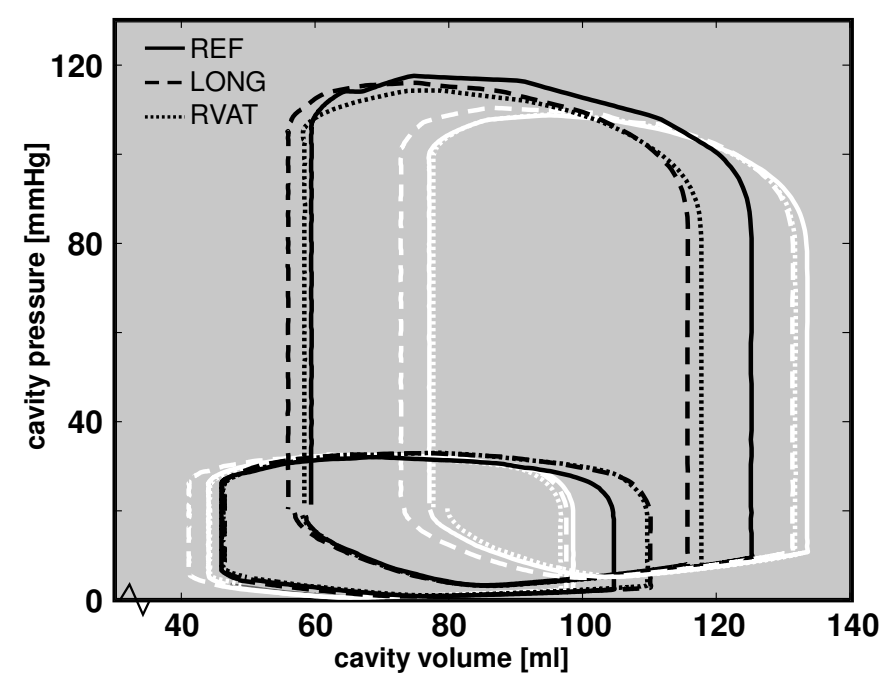

Figure 5.7: Adaptive myofiber reorientation causes an increase in pump function. Pressure-volume loops of the three geometries REF (solid), LONG (dashed), and RVAT (dotted). The white lines are the results for the initial state (init), where no myofiber reorientation has taken place yet. The black lines are the results for the adapted state (adap) after reorientation. 

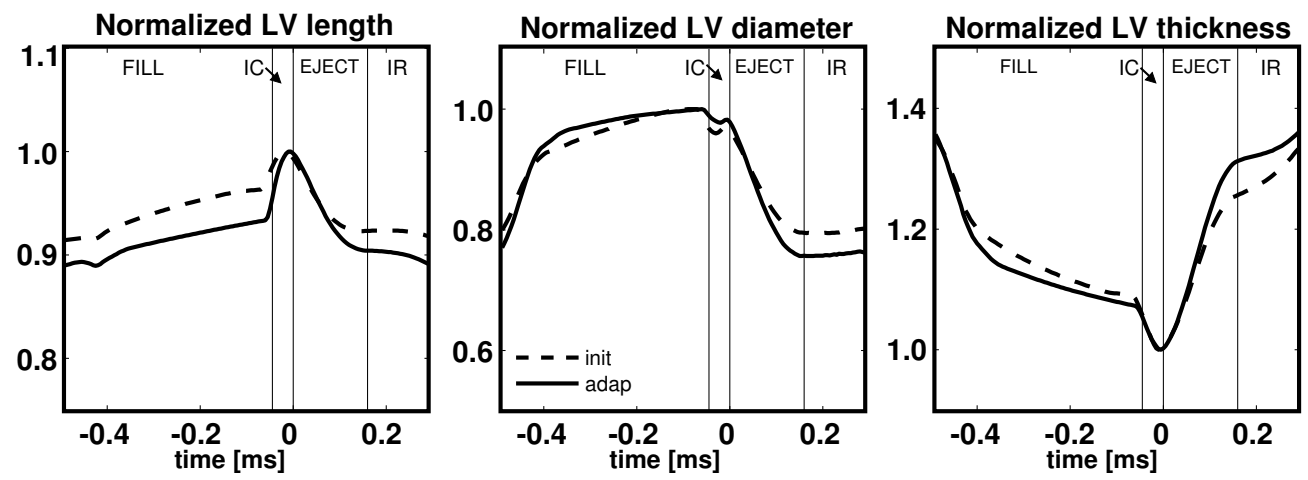

Figure 5.8: Change in LV inner length (left), in LV inner diameter at equatorial height (middle), and in LV wall thickness at equatorial height (right) in simulation REF during a cardiac cycle. During ejection, shortening in both diameter and length and wall thickening was increased in the adapted state (solid) compared to the initial state (dotted). FILL=filling phase; $I C=$ isovolumic contraction phase; $E J E C T=$ ejection phase; $I R=$ isovolumic relaxation phase.

results as compared to evaluation of global deformation, because $E_{c r}$ is not kinematically coupled to the cavity volume. In the experimental results [28], the total amplitude of $E_{c r}$ is $\sim 0.10$ and the gradient in $E_{c r}$ at end-ejection is base-mid-apex. In the initial state, the total amplitude of $E_{c r}$ is $\sim 0.35$ and it reduces to $\sim 0.15$ in the adapted state. In addition, the gradient in Ecr at endejection flips from apex-mid-base in the initial state to base-mid-apex in the adapted state. Again, a similar trend was observed in LONG and RVAT.

\subsection{Discussion}

In this study, we investigated the sensitivity of cardiac function for geometry and myofiber orientation in a BiV FE model of cardiac mechanics. A geometrical variation in the $\mathrm{LV}$ short-to-long axis ratio of $27 \%$ in simulation $L O N G$ resulted in an increase in total pump work of only $4 \%$. A variation in the RV length by $16 \%$ through variation in the location of the attachment of RV to LV in simulation RVAT resulted in a decrease in total pump work of only $2 \%$. On the other hand, a change in myofiber orientation over an average angle of about $8^{\circ}$, as induced by adaptive myofiber reorientation (equation 5.1), caused an increase in total pump work of about $20 \%$ in REF, $11 \%$ in $L O N G$, and $22 \%$ in RVAT. In addition, we found a better agreement between model computed and experimental data on shear deformation when myofiber orientation was adapted.

The increase in pump work upon adaptation caused a redistribution of the total blood volume over the different compartments of the circulation mo- 

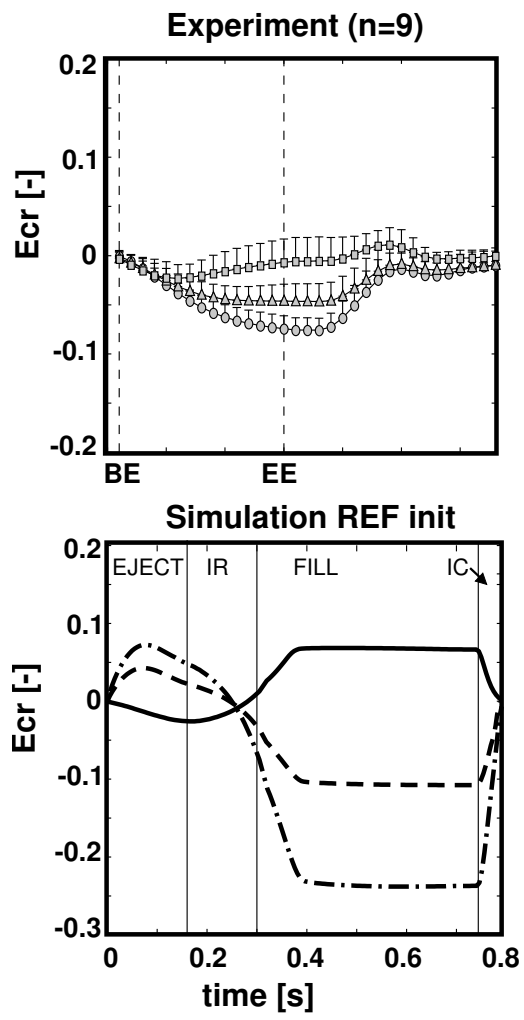
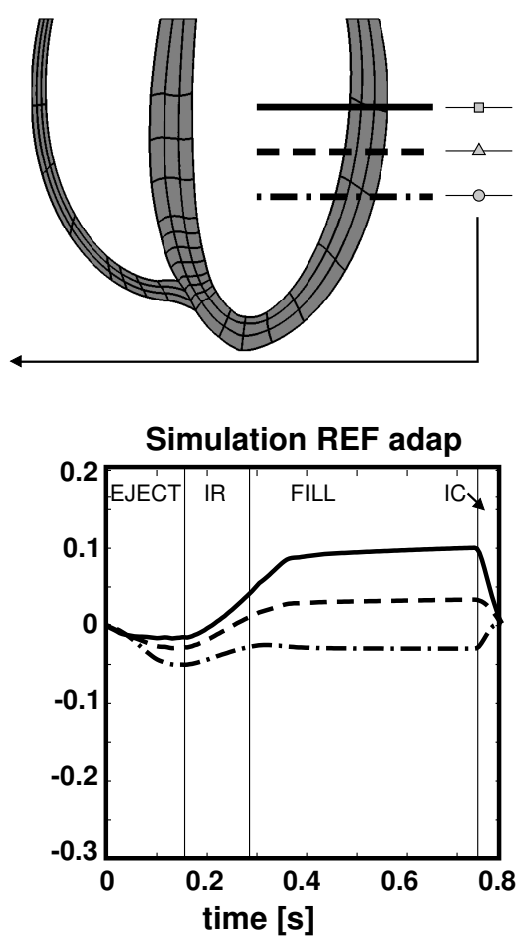

Figure 5.9: Comparison of experimental and model computed patterns of circumferential-radial shear $E_{c r}$. The average $E_{c r}$ (mean+SD) from nine healthy subjects as measured with MRT [8] is shown on the top left. The location of the MRslices relative to the model geometry at begin-ejection is shown on the top right. The bottom row shows model computed $E_{c r}$ of the REF simulation in the initial state (bottom, left) and in the adapted state (bottom, right). $B E=$ begin-ejection; $E E=$ endejection; $E J E C T=$ =jection phase; $I R=$ isovolumic relaxation phase; $F I L L=$ filling phase; $I C=$ isovolumic contraction phase. 
del. Consequently, LV end-diastolic pressure and volume were lower in the adapted state. A reduction in filling volume in itself would cause a reduction of end-diastolic sarcomere length, a reduction of ability to develop active stress ('Frank-Starling effect' [40]) and a decrease in pump function. The fact that pump function increases upon myofiber reorientation, despite the adverse Frank Starling effect, demonstrates the important role of myofiber orientation for efficient pump function.

Cardiac function Values of pump work in the adapted state lay slightly better within the physiological range than the values of pump work in the initial state $[13,33]$, and this was irrespective of the geometry. Similarly, a model predicted value for natural myofiber strain during ejection of about -0.15 (table 5.5) is in good agreement with experimental measurements [7]. Evaluation of active myofiber stress is more difficult because data on active myofiber stress is only available from in vitro isometric measurements [16, 29, 44]. Indirect evaluation of active myofiber stress is possible through evaluation of stroke work density $w_{f}$, calculated from the active myofiber Cauchy stressnatural strain loop. A value for total global pump $\left(W_{l v}+W_{r v}\right)$ of around 1.1 $\mathrm{J}$ is within the physiological range for a total ventricular wall volume of 200 $\mathrm{ml}$ [13]. Since total global pump work is equal to $w_{f}$ integrated over the total ventricular wall volume, we find a volume weighted average about $5.5 \mathrm{~mJ} / \mathrm{ml}$ for $w_{f}$. Note that this value differs slightly from the value in table 5.5 , because the representative volume differs between nodes depending on their location. Together with a myofiber strain of about -0.15 , a value of about $40 \mathrm{kPa}$ is expected to occur in the intact heart. Also, values for local function agreed with predictions from previous models developed in our group [2, 28].

Besides hemodynamic and myofiber measures, also global deformation of the biventricular model was analyzed to evaluate the model results. Longitudinal shortening of $10 \%$ during ejection seems to be in good agreement with experimental data [39]. Longitudinal and diameter shortening and radial thickening increased as a result of myofiber reorientation. This could also be observed by the increased RV and LV EFs. The adapted values for EF were closer to the physiological value for EF of about $60 \%$ [13].

Adaptive myofiber reorientation caused a decrease in myofiber strain during isovolumic contraction $\left(\epsilon_{f, i c}\right)$ and during isovolumic relaxation $\left(\epsilon_{f, i r}\right)$. Thus, the amount of shear deformation indeed decreased, indicating an increased match of the shape of the complete passive and completely activated heart. In addition, local myocardial function became more homogeneously distributed through adaptive myofiber reorientation as indicated by a decrease is most of the standard deviations of local myocardial function. An increased homogeneity of local function was furthermore observed by a decrease of the 
coefficients of variation $C_{v}$ in the adapted state (table 5.6). This increase in homogeneity is in agreement with hypotheses and measurements that local myocardial function is homogeneously distributed throughout the wall [23, 50].

Myofiber orientation The initial distribution for $\alpha_{h, 0}$ was based on a distribution that was already optimized for homogeneity of myofiber strain during ejection in the LV [31]. The start with an optimized distribution explains why changes in $\alpha_{h, 0}$ between the initial and adapted state were small. Experimental data show a similar transmural distribution for $\alpha_{h, 0}$ in the LV and RV [14, $25,26,52]$. Therefore, we used the same initial distribution for $\alpha_{h, 0}$ in the RV.

Critical testing of the distribution of $\alpha_{t, 0}$ is difficult because of the lack of accurate measurements on the spatial distribution of myofiber orientation. A difference in myofiber orientation of $8^{\circ}$ can hardly be detected with in vitro, let alone with in vivo DTMRI $[34,46]$. Nevertheless, adapted distributions for $\alpha_{t, 0}$ were very well within the range of experimental data (figure 5.6). Model predictions of the developed $\alpha_{t, 0}$ in the LV free wall (LVfw) agreed with predictions from our LV only model [28]. In addition, we observe a clear difference in $\alpha_{t, 0}$ in between the anterior and posterior septum (figure 5.5). The asymmetry between posterior and anterior is supported by results from DTMRI studies $[9,14]$.

Values of $\alpha_{t, 0}$ were significantly smaller in the RVfw as compared to the $\mathrm{LVfw}$ and septum. As the RVfw is thin as compared to the $\mathrm{LV}$ wall (ratio $f_{T}$ is 0.33 , see table 5.1), shear deformation will be lower in the RVfw than in the LV wall. This explains the different development of $\alpha_{t, 0}$ between the RVfw and LV wall.

Although experimental data confirm the presence of a transverse component in myofiber orientation [14, 21, 22, 41], many computational studies on cardiac mechanics only include the helical character of myofiber orientations $[18,24,32] . \alpha_{t, 0}$ is often excluded because these angles are considered small. This is indeed true near the equatorial region. However, values for $\alpha_{t, 0}$ increase up to $\sim 10^{\circ}$ in the apical and basal regions. This is still small compared to $\alpha_{h, 0}$, but we again showed that such small values for $\alpha_{t, 0}$ had a large effect on the pump function and circumferential-radial shear strain, and therefore, $\alpha_{t, 0}$ should not be ignored. Since experimental measurement of $\alpha_{t, 0}$ is difficult, reorientation of myofibers in response to fiber cross-fiber shear could serve as a method to refine the myofiber orientation in geometrically correct $\mathrm{BiV}$ models.

More recently, computational studies have been published that included both longitudinal and transmural component in myofiber orientation by mapping DTMRI data onto a mesh. Often a good agreement was reported between 
model computed and experimental data on hemodynamic function $[6,35]$ or electrophysiology [49]. It would be interesting to analyze cardiac deformation in these models, because in our model computed circumferential-radial shear was found to be very sensitive to the choice of myofiber orientation. Small changes in orientation, that might be difficult to detect with DTMRI, influenced especially shear deformation. Our computed results showed better agreement with experimental data both qualitatively (gradient in $E_{c r}$ ) as well as quantitatively (amplitude of $E_{c r}$ ) after adaptive myofiber reorientation.

Study assumptions and limitations Our parameterized description of the BiV geometry enabled well-defined variations of the geometry: the effect of geometrical characteristics was easily investigated by changing one of the parameter values listed in table 5.1. Moreover, wall and cavity volumes were easily kept constant. This was of importance, since it is known that the ratio of cavity-to-wall volume largely determines the mean level of active wall stress [2].

Results of the adapted state were analyzed after 20 cardiac cycles with adaptive myofiber reorientation. At this stage, myofiber reorientation has not yet reached a steady state: myofiber orientation still changed by about $1^{\circ}$ per cardiac cycle. Because local and global function did not change anymore, we considered the situation after 20 cycles of adaptation representative for the effect of adaptation.

Typically, systemic venous compliance $C_{s, v e n}$ is about thirty times higher than systemic arterial compliance $C_{s, a r t}$, while pulmonary venous compliance $C_{p, v e n}$ is about one third of $C_{s, \text { ven }}[38,37]$. With these parameter values, it took over 50 cardiac cycles to reach a hemodynamic steady state. Therefore, $C_{s, \text { ven }}$ and $C_{p \text {,ven }}$ were decreased ten times (see table 5.2), to ensure a faster feedback on a difference between RV and LV stroke volume. In addition, we increased unstressed venous volumes $\left(V_{\text {ven }, 0}\right)$ so that mean pressure levels remained unchanged. Although these parameter values might be outside the physiological range, it led to an on average equal RV and LV stroke volume and end-diastolic pressures remained the same.

In this chapter, a switch was made from the material model of Bovendeerd et al. [5] to the material model of Kerckhoffs et al. [17]. The latter model is more suited to capture the exponential increase of passive stress at sarcomere lengths higher than $2.2 \mu \mathrm{m}$ that has been observed in experiments [43]. Whereas such high sarcomere lengths did not occur in the present study, they might occur in future applications of the model, such as the study of the asynchronously activated heart. To be able to use the present computations as a reference for these future applications, we decided to change the material model already in this study. 


\subsection{Conclusion}

The results from the BiV FE model in this study showed that a geometrical variation of $27 \%$ in the LV short-to-long axis ration and a variation of $16 \%$ in $\mathrm{RV}$ length led to a change in total pump work of about $7 \%$. On the other hand, an average changes in myofiber orientation of about $8^{\circ}$ caused an increase in total pump work of about $18 \%$ in all three geometries. Differences in total pump work between the three geometries with adapted myofiber orientation were below $1 \%$. In addition, circumferential-radial shear strain showed better agreement with experimental data when myofiber orientation was adapted. These findings indicate the importance for a thorough effort to address a realistic myofiber orientation. As current techniques for (in vivo) measurement of myofiber orientation lack sufficient accuracy, the currently used model for remodeling of myofiber orientation seems a useful approach to set myofiber orientations in $\mathrm{BiV}$ FE models, before optimizing myocardial parameters to arrive at a patient-specific model. 


\section{References}

1. Aguado-Sierra J, Krishnamurthy A, Villongco C, Chuang J, Howard E, Gonzales MJ, Omens J, Krummen DE, Narayan S, Kerckhoffs RCP, and McCulloch AD (2011). "Patientspecific modeling of dyssynchronous heart failure: a case study." Prog Biophys Mol Biol 107.1, pp. 147-155.

2. Arts T, Bovendeerd PH, Prinzen FW, and Reneman RS (1991). "Relation between left ventricular cavity pressure and volume and systolic fiber stress and strain in the wall." Biophys J 59.1, pp. 93-102.

3. Arts T, Prinzen FW, Snoeckx LH, Rijcken JM, and Reneman RS (1994). "Adaptation of cardiac structure by mechanical feedback in the environment of the cell: a model study". Biophysical Journal 66, pp. 953-961.

4. Bayer JD, Blake RC, Plank G, and Trayanova NA (2012). "A novel rule-based algorithm for assigning myocardial fiber orientation to computational heart models." Ann Biomed Eng 40.10, pp. 2243-2254.

5. Bovendeerd PHM, Kroon W, and Delhaas T (2009). "Determinants of left ventricular shear strain". Am J Physiol - Heart Circ Physiol 297.3, H1058-H1068.

6. Constantino J, Hu Y, and Trayanova NA (2012). "A computational approach to understanding the cardiac electromechanical activation sequence in the normal and failing heart, with translation to the clinical practice of CRT.". Prog Biophys Mol Biol 110.2-3, pp. 372-379.

7. Delhaas T, Arts T, Bovendeerd P, Prinzen F, and Reneman R (1993). "Subepicardial fiber strain and stress as related to left ventricular pressure and volume". Am J Physiol-Heart Circ Physiol 264, H1548-H1559.

8. Delhaas T, Kroon W, Decaluwe W, Rubbens M, Bovendeerd P, and Arts T (2008). "Structure and torsion of the normal and situs inversus totalis cardiac left ventricle; Part I: experimental data in humans". Am J Physiol - Heart Circ Physiol 295, H197-H201.

9. Geerts-Ossevoort L, Bovendeerd P, Nicolay K, and Arts T (2002). "Characterization of the normal cardiac myofiber field in goat measured with MR-diffusion tensor imaging". Am J Physiol - Heart Circ Physiol 283.1, H139-H145.

10. Geerts-Ossevoort L, Kerckhoffs R, Bovendeerd P, and Arts T (2003). “Towards Patient Specific Models of Cardiac Mechanics: A Sensitivity Study". In: Functional Imaging and Modeling of the Heart. Ed. by I Magnin, J Montagnat, P Clarysse, J Nenonen, and T Katila. Vol. 2674. Lecture Notes in Computer Science. Springer Berlin Heidelberg, pp. 81-90.

11. Greenbaum R, Ho S, Gibson D, Becker A, and Anderson R (1981). "Left ventricular fibre architecture in man". Br Heart J 45, pp. 248-263.

12. Gurev V, Lee T, Constantino J, Arevalo H, and Trayanova NA (2011). "Models of cardiac electromechanics based on individual hearts imaging data: image-based electromechanical models of the heart." Biomech Model Mechanobiol 10.3, pp. 295-306.

13. Guyton AC and Hall JE (2006). Textbook of medical physiology. Elsevier Saunders.

14. Helm P, Beg MF, Miller MI, and Winslow RL (2005). "Measuring and mapping cardiac fiber and laminar architecture using diffusion tensor MR imaging." Ann N Y Acad Sci 1047, 
pp. 296-307.

15. Hsu EW, Muzikant AL, Matulevicius SA, Penland RC, and Henriquez CS (1998). "Magnetic resonance myocardial fiber-orientation mapping with direct histological correlation." Am J Physiol 274.5, H1627-H1634.

16. Janssen PM and Hunter WC (1995). "Force, not sarcomere length, correlates with prolongation of isosarcometric contraction." Am J Physiol 269.2 Pt 2, H676-H685.

17. Kerckhoffs RCP, Bovendeerd PHM, Kotte JCS, Prinzen FW, Smits K, and Arts T (2003).

"Homogeneity of cardiac contraction despite physiological asynchrony of depolarization: a model study." Ann Biomed Eng 31.5, pp. 536-547.

18. Kerckhoffs RCP, Healy SN, Usyk TP, and D MA (2006). "Computational Methods for Cardiac Electromechanics". J PROC IEEE 94.4, pp. 769-783.

19. Kroon W, Delhaas T, Arts T, and Bovendeerd P (2009). "Computational analysis of the myocardial structure: Adaptation of myofiber orientations through deformation in three dimensions". Med Imag Anal 13, pp. 346-353.

20. Lamata P, Sinclair M, Kerfoot E, Lee A, Crozier A, Blazevic B, Land S, Lewandowski AJ, Barber D, Niederer S, and Smith N (2014). "An automatic service for the personalization of ventricular cardiac meshes." J R Soc Interface 11.91, p. 20131023.

21. Legrice IJ, Hunter PJ, and Smaill BH (1997). "Laminar structure of the heart: a mathematical model." Am J Physiol 272.5, H2466-H2476.

22. Lunkenheimer PP, Redmann K, Dietl KH, Cryer C, Richter KD, Whimster WF, and Niederer P (1997). “The heart's fibre alignment assessed by comparing two digitizing systems. Methodological investigation into the inclination angle towards wall thickness." Technol Health Care 5.1-2, pp. 65-77.

23. MacGowan GA, Shapiro EP, Azhari H, Siu CO, Hees PS, Hutchins GM, Weiss JL, and Rademakers FE (1997). "Noninvasive measurement of shortening in the fiber and cross-fiber directions in the normal human left ventricle and in idiopathic dilated cardiomyopathy". Circulation 96, pp. 535-541.

24. Niederer SA, Plank G, Chinchapatnam P, Ginks M, Lamata P, Rhode KS, Rinaldi CA, Razavi R, and Smith NP (2011). "Length-dependent tension in the failing heart and the efficacy of cardiac resynchronization therapy". Cardiovascular Research 89(2), pp. 336-343.

25. Nielsen E, Smerup M, Agger P, Frandsen J, Ringgard S, Pedersen M, Vestergaard P, Nyengaard JR, Andersen JB, Lunkenheimer PP, Anderson RH, and Hjortdal V (2009). "Normal right ventricular three-dimensional architecture, as assessed with diffusion tensor magnetic resonance imaging, is preserved during experimentally induced right ventricular hypertrophy." Anat Rec (Hoboken) 292.5, pp. 640-651.

26. Nielsen PM, LeGrice IJ, Smaill BH, and Hunter PJ (1991). "Mathematical model of geometry and fibrous structure of the heart". Am J Physiol - Heart Circ Physiol 260, H1365-H1378.

27. Peyrat JM, Sermesant M, Pennec X, Delingette H, Xu C, McVeigh ER, and Ayache N (2007). "A computational framework for the statistical analysis of cardiac diffusion tensors: application to a small database of canine hearts." IEEE Trans Med Imaging 26.11, pp. 1500-1514. 28. Pluijmert M, Bovendeerd PHM, Kroon W, Prinzen FW, and Delhaas T (2014). "Effects of activation pattern and active stress development on myocardial shear in a model with adaptive myofiber reorientation." Am J Physiol Heart Circ Physiol 306.4, H538-H546.

29. Pollack GH and Krueger JW (1976). "Sarcomere dynamics in intact cardiac muscle." Eur J Cardiol 4 Suppl, pp. 53-65.

30. Reese TG, Weisskoff RM, Smith RN, Rosen BR, Dinsmore RE, and Wedeen VJ (1995). "Imaging myocardial fiber architecture in vivo with magnetic resonance." Magn Reson Med 34.6, pp. 786-791.

31. Rijcken JM, Bovendeerd PHM, Schoofs AJG, Van Campen DH, and Arts T (1999). “Optimization of cardiac fiber orientation for homogeneous fiber strain during ejection". Ann Biomed Eng 27, pp. 289-297. 
32. Saint-Marie J, Chapelle D, Cimrman R, and Sorine M (2006). "Modeling and estimation of the cardiac electromechanical activity". Comp and Struc 84, pp. 1743-1759.

33. Schlosser T, Pagonidis K, Herborn CU, Hunold P, Waltering KU, Lauenstein TC, and Barkhausen J (2005). “Assessment of left ventricular parameters using 16-MDCT and new software for endocardial and epicardial border delineation." AJR Am J Roentgenol 184.3, pp. 765773.

34. Scollan DF, Holmes A, Winslow R, and Forder J (1998). "Histological validation of myocardial microstructure obtained from diffusion tensor magnetic resonance imaging." Am $J$ Physiol 275.6, H2308-H2318.

35. Sermesant M, Chabiniok R, Chinchapatnam P, Mansi T, Billet F, Moireau P, Peyrat JM, Wong K, Relan J, Rhode K, et al. (2012). "Patient-specific electromechanical models of the heart for the prediction of pacing acute effects in CRT: a preliminary clinical validation." Med Image Anal 16.1, pp. 201-215.

36. Sermesant M, Billet F, Chabiniok R, Mansi T, Chinchapatnam P, Moireau P, Peyrat JM, Rhode K, Ginks M, Lambiase P, et al. (2009). "Personalised Electromechanical Model of the Heart for the Prediction of the Acute Effects of Cardiac Resynchronisation Therapy". In: Functional Imaging and Modeling of the Heart. Ed. by N Ayache, H Delingette, and M Sermesant. Vol. 5528. Lecture Notes in Computer Science. Springer Berlin Heidelberg, pp. 239-248.

37. Shoukas AA (1975). "Pressure-flow and pressure-volume relations in the entire pulmonary vascular bed of the dog determined by two-port analysis." Circ Res 37.6, pp. 809818.

38. Shoukas AA and Sagawa K (1973). “Control of total systemic vascular capacity by the carotid sinus baroreceptor reflex." Circ Res 33.1, pp. 22-33.

39. Simonson JS and Schiller NB (1989). "Descent of the base of the left ventricle: an echocardiographic index of left ventricular function." J Am Soc Echocardiogr 2.1, pp. 25-35.

40. Starling EH (1918). The Linacre Lecture on the Law of the Heart. London, Longmans, Green \& Co.

41. Streeter Jr. DD (1978). “Cardiovascular System Dynamics". In: ed. by J Baan, A Noordergraaf, and J Raines. Cambridge, MA: MIT. chap. Three-dimensional fiber orientation in mammalian left ventricular wall, pp. 73-84.

42. Streeter Jr D, Spotnitz HM, Patel DP, Ross Jr J, and Sonnenblick EH (1969). "Fiber orientation in the canine left ventricle during diastole and systole." Circ Res 24.3, pp. 339-347.

43. Stuyvers BD, McCulloch AD, Guo J, Duff HJ, and Keurs HEDJ ter (2002). "Effect of stimulation rate, sarcomere length and $\mathrm{Ca}(2+)$ on force generation by mouse cardiac muscle." J Physiol 544.Pt 3, pp. 817-830.

44. ter Keurs HE, Rijnsburger WH, van Heuningen R, and Nagelsmit MJ (1980). "Tension development and sarcomere length in rat cardiac trabeculae. Evidence of length-dependent activation." Circ Res 46.5, pp. 703-714.

45. Toussaint N, Sermesant M, Stoeck CT, Kozerke S, and Batchelor PG (2010). "In vivo human 3D cardiac fibre architecture: reconstruction using curvilinear interpolation of diffusion tensor images." Med Image Comput Comput Assist Interv 13.Pt 1, pp. 418-425.

46. Tseng WY, Reese TG, Weisskoff RM, and Wedeen VJ (1999). “Cardiac diffusion tensor MRI in vivo without strain correction." Magn Reson Med 42.2, pp. 393-403.

47. Usyk TP, Mazhari R, and McCulloch AD (2000). "Effect of laminar orthotropic myofiber architecture on regional stress and strain in the canine left ventricle". J Elasticity 61, pp. 143164.

48. Vadakkumpadan F, Arevalo H, Ceritoglu C, Miller M, and Trayanova N (2012). “Imagebased estimation of ventricular fiber orientations for personalized modeling of cardiac electrophysiology." IEEE Trans Med Imaging 31.5, pp. 1051-1060.

49. Vadakkumpadan F, Arevalo H, and Trayanova NA (2013). "Patient-specific modeling of the heart: estimation of ventricular fiber orientations." J Vis Exp 71. 
50. van der Vusse GJ, Arts T, Glatz JF, and Reneman RS (1990). “Transmural differences in energy metabolism of the left ventricular myocardium: fact or fiction." J Mol Cell Cardiol 22.1, pp. 23-37.

51. Xia L, Huo M, Wei Q, Liu F, and Crozier S (2005). “Analysis of cardiac ventricular wall motion based on a three-dimensional electromechanical biventricular model." Phys Med Biol 50.8, pp. 1901-1917.

52. Zhang L, Allen J, Hu L, Caruthers SD, Wickline SA, and Chen J (2013). “Cardiomyocyte architectural plasticity in fetal, neonatal, and adult pig hearts delineated with diffusion tensor MRI.". Am J Physiol Heart Circ Physiol 304.2, H246-H252. 


\section{Chapter 6}

\section{Left ventricular pacing site and response to CRT}

In preparation:

M. Pluijmert, P.H.M. Bovendeerd, J. Lumens, K. Vernooy, T. Delhaas, F.W. Prinzen, How the left ventricular pacing site determines response to cardiac resynchronization therapy: a computational study 


\section{Abstract}

Cardiac resynchronization therapy (CRT) produces clinical benefits in chronic heart failure patients with left bundle branch block (LBBB). The position of the pacing site on the left ventricle (LV) is considered an important determinant of CRT response, but the mechanism how the LV pacing site determines CRT response is not completely understood. Therefore, we investigated the relation between $\mathrm{LV}$ pacing site during biventricular $(\mathrm{BiV})$ pacing and cardiac function in a finite element model of LV and right ventricular electromechanics. Cardiac function, assessed as LV $d p / d t_{\max }$ and stroke work, was evaluated during normal electrical activation, typical LBBB, fascicular blocks and BiV pacing with different $L V$ pacing sites. Cardiac function correlated closely with fast recruitment of $\mathrm{LV}$ activation, low LVfw-septal dyssynchrony, and intra $\mathrm{LV}$ dyssynchrony. The best cardiac function was obtained when pacing the midlevel LV lateral wall. This site was close to the latest activated region (LAR) for the simulation of typical LBBB, but not for the fascicular block simulations. The largest hemodynamic response did not coincide with largest reduction in total activation time (comparable to QRS duration). This study indicates that the best hemodynamic response to CRT is achieved by fast recruitment of LV activation, leading to maximum reduction in inter and intra LV dyssynchrony. This is achieved by pacing a central location on the LV lateral wall. 


\subsection{Introduction}

Cardiac resynchronization therapy (CRT) produces clinical benefits in chronic heart failure patients with reduced left ventricular ejection fraction and left bundle branch block (LBBB) [1,6]. LBBB causes delayed activation of the left ventricular free wall ( $\mathrm{LVfw}$ ) relative to the septum, and consequently, dyssynchronous myocardial contraction and diminished cardiac pump function. In CRT, biventricular (BiV) pacing is employed to resynchronize the electrical activation and thereby to restore a more synchronized contraction of the ventricles.

The position of the pacing site on the LVfw is considered one of the important determinants of CRT response [17, 28, 29, 32]. Current guidelines recommend to place the LV electrode, whenever possible, in a specific anatomical region, i.e. the postero-lateral region and away from the apex [33]. However, some studies report no significant difference in response to CRT when pacing from various circumferential and longitudinal positions on the $\operatorname{LVfw}[9,11$, 18 , 27]. In addition, the region that resulted in the best response is inconsistent between studies [10, 21, 28, 29]. Alternatively, it has been proposed to position the LV electrode in the latest activated region (LAR) during intrinsic activation. Indeed, a positive correlation was found between the activation time in the LAR and response to CRT when pacing from this site [12, 17, 34]. However, most of this evidence has been derived from comparing single pacing sites between patients, rather than comparing the effect of multiple pacing sites in the same patients and if so, only a few sites were accessible [36].

The aim of this study was to investigate the relation between electrical dyssynchrony, and cardiac function in a finite element (FE) model of left and right ventricular electromechanics. We assessed cardiac function during normal electrical activation, different types of LBBB, and BiV pacing with different LV pacing sites. Subsequently, we investigated which LV pacing site resulted in maximum improvement of cardiac function.

\subsection{Methods}

The computational model of cardiac mechanics has been presented in chapter 5 and a summary will be given here.

\section{Model of Cardiac Mechanis}

The right and left ventricle (RV and LV, respectively) were approximated by two intersecting ellipsoids. RV and LV wall volumes were set to $40 \mathrm{ml}$ and 160 $\mathrm{ml}$, respectively (figure 6.1). A realistic myofiber orientation with a longitudinal and transmural component was created (chapter 5) by local adaptation 
of the myofiber orientation in response to fiber cross-fiber shear [19]. Myocardial mechanical tissue properties were described by means of a passive and an active part. The passive myocardium was assumed nonlinearly elastic, transversely isotropic, and nearly incompressible. The passive stress-strain relation was modeled with the strain energy density function as described in [16]. The magnitude of active stress depended on time elapsed since activation, sarcomere length, and sarcomere shortening velocity as described in [3]. Pre- and afterload were simulated by the interaction of the heart with a lumped parameter model of the circulation (figure 6.1). Parameter values for the circulation model can be found in table 6.1.

\section{Model of Electrical Wave Propagation}

The spatial distribution of the electrical activation time $A T$ in each nodal position was determined by solving the Eikonal-diffusion equation in the enddiastolic configuration [16]. Computed ATs were used to initiate development of active stress in the model of cardiac mechanics.

In the normal case (NORM), activation started $(A T=0 \mathrm{~ms})$ from five Purkinje exit points defined at both the RV and LV endocardium (figure 6.2, left). These exit points were based on three-dimensional mapping studies in the human heart [7]. From the exit points, activation propagated throughout the rest of the myocardium. Propagation was assumed anisotropic with a conduction velocity of $0.70 \mathrm{~m} / \mathrm{s}$ along the myofiber direction $\left(c_{f, \text { myo }}\right)$ and $0.30 \mathrm{~m} / \mathrm{s}$ transverse to the myofiber direction $\left(c_{t, m y o}\right)$ [5]. In addition, conduction velocity was assumed higher at the endocardium where it was set to $2.08 \mathrm{~m} / \mathrm{s}$ in myofiber direction $\left(c_{f, e n}\right)$ and to $1.59 \mathrm{~m} / \mathrm{s}$ transverse to the myofiber direction $\left(c_{t, e n}\right)[26,30]$. Velocities decreased linearly from endocardial to myocardial value over a relative transmural depth of $20 \%$.

\section{Numerical implementation}

Computations of biventricular mechanics were performed in a mesh that consisted of 684 27-noded hexahedral elements resulting in an average spatial resolution of $3 \mathrm{~mm}$. The Eikonal-diffusion equations were solved in a denser mesh that consisted of 29760 8-noded hexahedral elements with an average spatial resolution of less than $1 \mathrm{~mm}$. ATs that were computed in the denser mesh were projected on the coarser mesh.

\section{Simulations}

Starting from a reference simulation representing the normal heart (REF simulation in Chapter 5), we simulated three cases of LBBB, and 16 cases of BiV 
pacing each with a different LV electrode position. The heart rate was fixed at 75 beats per minute.

LBBB was modeled by a global $20 \%$ reduction of conduction velocities with respect to NORM [2]. In addition, transmural conduction in the septum was further decreased by $15 \%$ at the RV endocardial side, and by $60 \%$ in the midmyocardium and LV endocardial side [31]. Exit points were only located at the RV endocardium (figure 6.2, middle left). In addition, two cases of incomplete LBBB were created with a more distal block in the left bundle branch. The latter two baseline situations represented a left anterior ( $L A F B)$ and posterior $(L P F B)$ fascicular block by allowing one exit point at the posterior or anterior side of the LV endocardium, respectively.

CRT was simulated by point stimulation at the RV endocardial apex with simultaneous stimulation from one of 16 locations that were distributed over the $\mathrm{LV}$ free wall (LVfw) (figure 6.2, middle right). Thereby, we assumed that during $\mathrm{BiV}$ pacing the ventricular activation is dominated by the activation wavefronts originating from the two pacing sites, i.e., no fusion with intrinsic activation. An example activation pattern during $\mathrm{BiV}$ pacing is presented in the right panel of figure 6.2.

In a first step, we evaluated the electrical activation in terms of cumulative activation of the LVfw, because especially this part of the ventricles is likely to be influenced by the LV electrode position and this metric accurately expressed timing and temporal variation of activation of this wall. In addition, we evaluated total LV $A T\left(T A T_{l v}\right)$ and total $A T$ of both ventricles TAT (i.e., metric for QRS duration).

Next, we investigated the relation between more clinically used measures of electrical dyssynchrony and cardiac function [8, 24]. Therefore, dyssynchrony was defined as inter-wall dyssynchrony (difference in mean AT) between 1) LVfw and septum, and 2) LVfw and RVfw, and intra-wall dyssynchrony (standard deviation (SD) of $A T$ ) in the 3) entire LV and 4) LVfw only. Cardiac function was quantified by maximum rate of rise of LV pressure (LV $\left.d p / d t_{\max }\right)$ and by LV stroke work (SW, area enclosed by the pressure-volume relation). After simulation 10 cycles to allow hemodynamic stabilization, mean and SD of hemodynamic data were computed from the following 10 subsequent cardiac cycles.

\subsection{Results}

Variation in electrical activation caused differences in pump function, as shown in figure 6.3. In the $\angle B B B$ simulation, maximum LV pressure, stroke volume, and thus LV SW decreased with respect to the NORM simulation. BiV pacing with the LV pacing site at the mid-level antero-lateral wall partly restored LV 
Figure 6.1: The ellipsoidally shaped biventricular finite element (FE) mesh geometry for mechanical simulations. It consists of 684 elements and is incorporated in a lumped parameter model of the circulation. The valves are modeled as ideal diodes. Blood flow and storage of blood in both pulmonary and systemic circulation is modeled using resistances and compliances.

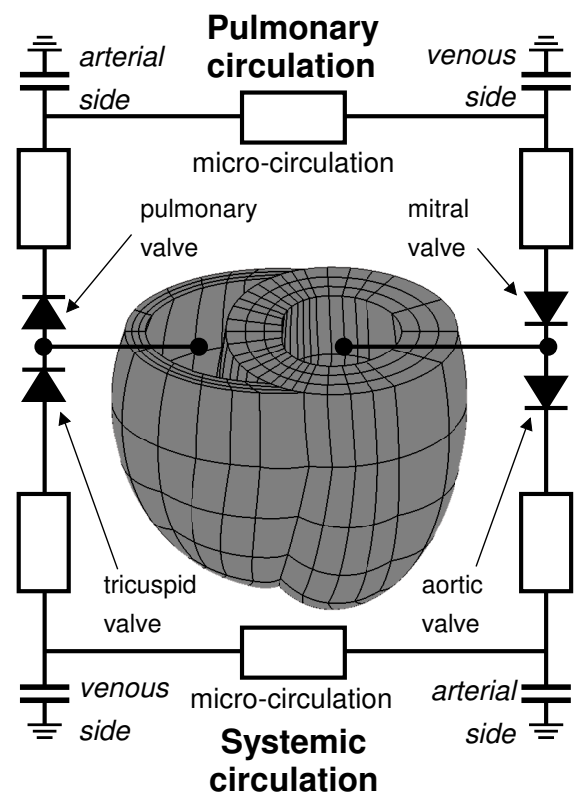

Table 6.1: Parameter values for the pulmonary and systemic circulation.

\begin{tabular}{lllll}
\hline parameter & definition & unit & systemic & pulmonary \\
\hline \hline$R_{\text {art }}$ & outflow resistance & {$\left[\mathrm{kPa} \cdot \mathrm{s} \cdot \mathrm{ml}^{-1}\right]$} & $4.46 \cdot 10^{-3}$ & $2.48 \cdot 10^{-3}$ \\
$R_{\text {per }}$ & peripheral resistance & {$\left[\mathrm{kPa} \cdot \mathrm{s} \cdot \mathrm{ml}^{-1}\right]$} & $1.49 \cdot 10^{-1}$ & $1.78 \cdot 10^{-2}$ \\
$R_{\text {ven }}$ & inflow resistance & {$\left[\mathrm{kPa} \cdot \mathrm{s} \cdot \mathrm{ml}^{-1}\right]$} & $1.10 \cdot 10^{-3}$ & $2.18 \cdot 10^{-3}$ \\
$C_{\text {art }}$ & arterial compliance & {$\left[\mathrm{ml} \cdot \mathrm{kPa}^{-1}\right]$} & $1.53 \cdot 10^{1}$ & $4.59 \cdot 10^{1}$ \\
$C_{\text {ven }}$ & venous compliance & {$\left[\mathrm{ml} \cdot \mathrm{kPa}^{-1}\right]$} & $4.59 \cdot 10^{1}$ & $1.53 \cdot 10^{1}$ \\
$V_{\text {art }, 0}$ & arterial unstressed volume & {$[\mathrm{ml}]$} & $7.04 \cdot 10^{2}$ & $7.83 \cdot 10^{1}$ \\
$V_{\text {ven }, 0}$ & venous unstressed volume & {$[\mathrm{ml}]$} & $3.16 \cdot 10^{3}$ & $5.13 \cdot 10^{2}$ \\
$V_{\text {tot }}$ & total blood volume & {$[\mathrm{ml}]$} & $5.00 \cdot 10^{3}$ & \\
\hline
\end{tabular}




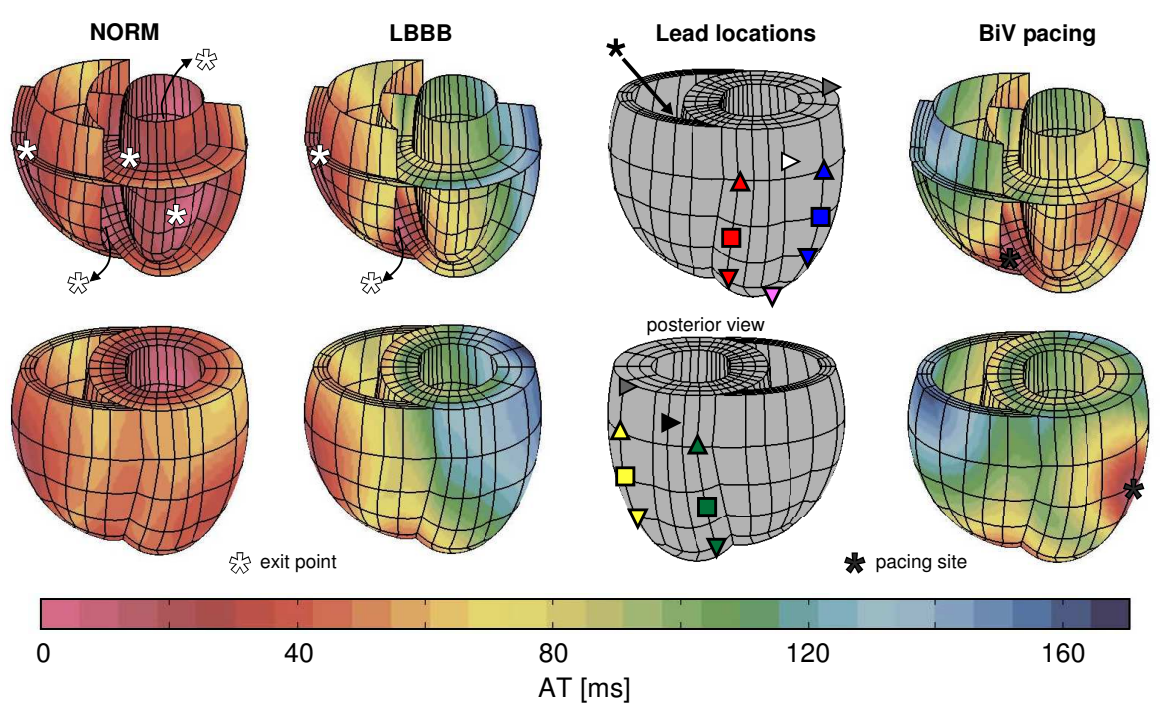

Figure 6.2: Activation times $(A T)$ in the right and left ventricle (RV and LV, respectively). Distributions of $A T$ are shown without (top row) and with (bottom row) epicardium. In the normal situation (NORM, left), activation starts in five exit points (white stars) at both the RV and LV endocardium. During $L B B B$ (middle left) activation only started in the RV. Cardiac resynchronization therapy (CRT) is simulated by point stimulation at the RV endocardial apex and simultaneous stimulation at one of the locations on the LV free wall ( $\mathrm{LVfw}$ ) as indicated by the symbols (middle right). An example of the activation pattern during $\mathrm{BiV}$ pacing with the $\mathrm{LV}$ pacing site at the mid-level antero-lateral wall is shown on the right.

SW towards NORM. The right panels of figure 6.3 show M-mode echocardiographic wall motion patterns. The inward motion of LVfw and septum occurred simultaneously in NORM. During $\angle B B B$, the motion pattern showed early septal inward motion ('septal flash'), which disappeared during CRT.

The advantage of the computer model as compared to measurements in animals and patients is that the onset of contraction can be calculated for all locations within the ventricles. This allowed a comprehensive analysis of the cumulative activation of ventricular myocardium during the various sequences of ventricular activation. The shape of the cumulative activation curve resembles that of a pharmacological dose-response curve and can be analyzed accordingly. The activation was characterized by the activation time of $50 \%$ of the LVfw myocardium $t_{50}$ and the speed of activation recruitment at this point $m_{t 50}$ (figure 6.4).

During normal activation (figure 4: white circles), the direct activation of the LVfw from the exit points of the fast conducting endocardium, resulted in immediate activation in the LVfw with fast increase $\left(t_{50}=30 \mathrm{~ms}\right.$, $m_{t 50}=3.1 \% / \mathrm{ms}$ ). In $\angle B B B$ (figure 4: grey circles), delayed activation of the 
LVfw caused a significantly larger $t_{50}(105 \mathrm{~ms})$. The slow progression of activation within the LVfw due to decreased conduction velocities and absence of Purkinje-system exit points in the LV, caused a more than 50\% reduction of $m_{t 50}(1.4 \% / \mathrm{ms})$. The various modes of $\mathrm{BiV}$ pacing (figure $4:$ squares and triangles) led to lower $t_{50}$ and higher $m_{t 50}$ values, but the extent of these changes depended on the specific site of pacing the LV. Largest reductions in $t_{50}$ were observed when pacing the mid-level of the antero- and postero-lateral wall (AL and PL, respectively). Largest increases in $m_{t 50}$ were observed when pacing the latest activated region, the mid-level AL wall, or the base of the PL wall. Moreover, $t_{50}$ showed a stronger correlation with $\mathrm{LV} d p / d t_{\max }$ than $m_{t 50}$.

When relating $\mathrm{LV} d p / d t_{\max }$ to clinically used indices of electrical dyssynchrony, a strong correlation was observed between $\mathrm{LV} d p / d t_{\max }$ and the LVfwseptum dyssynchrony. The LVfw-RVfw dyssynchrony was small during all $\mathrm{BiV}$ pacing modes, because the RV pacing component generated asynchronous activation within the RVfw with late activated regions in the RV base (see right panel in figure 6.2). As a consequence of the small LVfw-RVfw dyssynchrony during $\mathrm{BiV}$ pacing a poor correlation existed between $\mathrm{LV} d p / d t_{\max }$ and the LVfw-RVfw dyssynchrony (figure 6.5, left column). Fair correlations were found for the relation of intra-LV and intra-LVfw dyssynchrony and LV $d p / d t_{\max }$ (figure 6.5, right column).

Figure 6.6 presents the relation between LV pacing site and change in LV $d p / d t_{\text {max }}, \mathrm{LV} \mathrm{SW}$, and resynchronization, the latter expressed as decrease in $T A T_{l v}$ and as decrease in TAT. The location of LV pacing site that provided maximum hemodynamic effect was the mid-basal LV lateral wall. Importantly, a beneficial effect for LV $d p / d t_{\max }$ was observed at many LV pacing sites. Sites providing better hemodynamic response corresponded with those increasing LV stroke work and resynchronization of the LV, i.e. reducing $T A T_{l v}$, but not with those reducing TAT (metric for QRS duration).

The hypothesis that LV pacing in the LAR is an important determinant for effect of CRT was tested by plotting the relation between change in LV $d p / d t_{\max }$ during $\mathrm{BiV}$ pacing and the activation delay of the $\mathrm{LV}$ pacing site at baseline (figure 6.7). When using $\angle B B B$ as baseline, a statistically significant, but not perfect relation was found $(\mathrm{R}=0.78, \mathrm{p}<0.001)$. It can be observed that, at a given $A T$, the mid-level AL and PL pacing sites performed better than some later activated regions.

To further investigate the above hypothesis, we investigated the relation between change in LV $d p / d t_{\max }$ and the activation delay of the LV pacing site during the two other baseline situations of anterior and posterior fascicular blocks. Under these baseline conditions a very poor relation existed between $A T$ during intrinsic activation and increase in $\mathrm{LV} d p / d t_{\text {max }}$ during BiV pacing. Moreover, the extent of increase was smaller than during $L B B B$ (figure 6.7 middle and left panel). The on average smaller effect of $\mathrm{BiV}$ pacing can be ex- 
plained by the higher baseline LV $d p / d t_{\max }$ during the fascicular blocks. The relation between $A T$ and hemodynamic response was not significant in case of $\operatorname{LAFB}(\mathrm{R}=0.11, \mathrm{p}=0.68)$ and $\operatorname{LPFB}(\mathrm{R}=0.21, \mathrm{p}=0.43)$. In this regard it should be noted that during $\mathrm{BiV}$ pacing the ventricular activation is dominated by the activation wavefronts originating from the two pacing sites, which makes ventricular activation during $\mathrm{BiV}$ pacing independent of the baseline activation sequence.
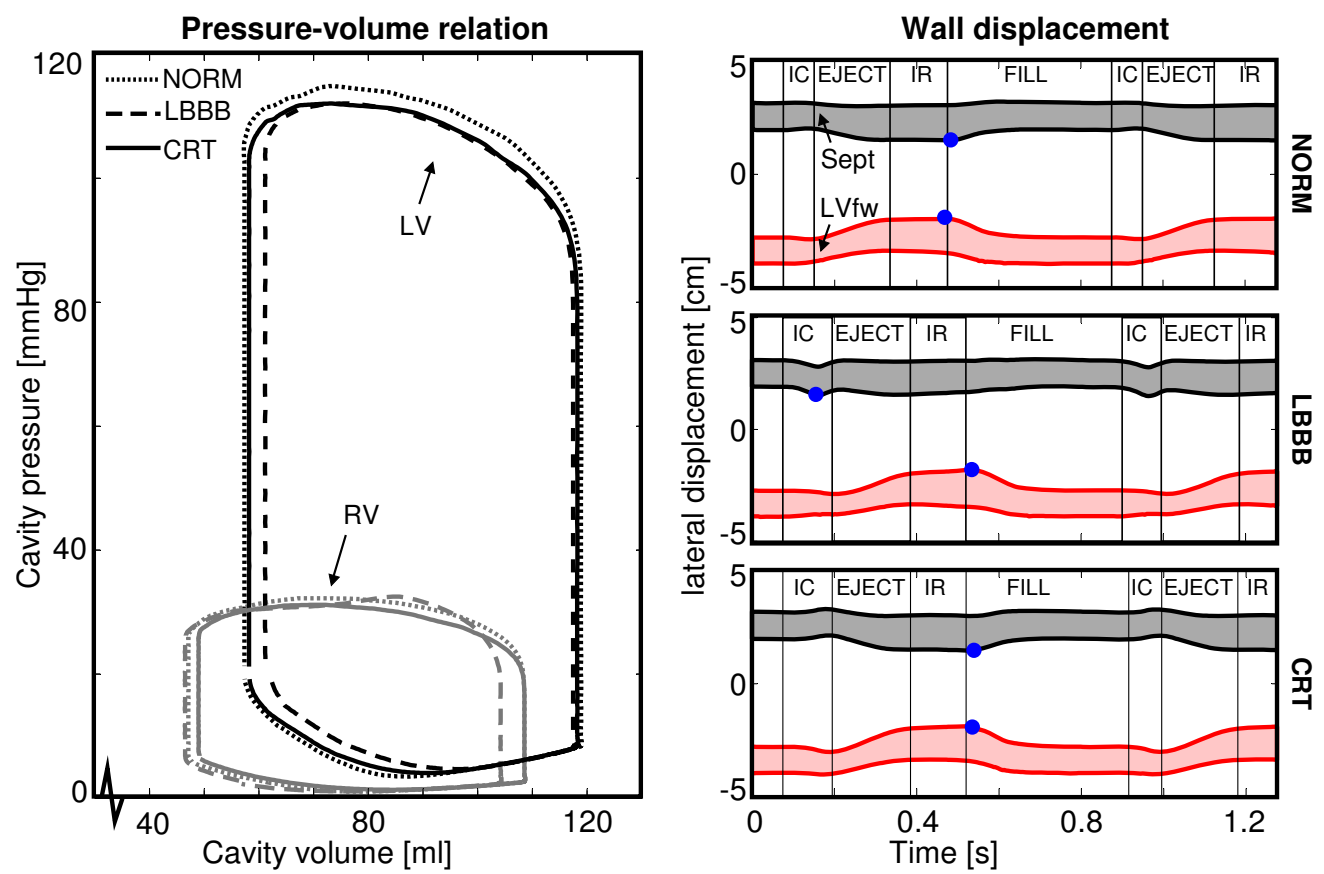

Figure 6.3: Simulated pressure-volume relations (left) and M-mode echocardiographic wall motion patterns (right). Left: LV (black) and RV (grey) pressure-volume relation from NORM (dotted), LBBB ( $L B B B$, dashed), and CRT with the LV pacing site at the mid-level antero-lateral wall are shown. Right: M-mode echocardiographic wall motion pattern of septum (Sept) and LV free wall (LVfw) at equatorial level. Blue dots indicate the moment of maximum inward motion. IC=isovolumic contraction; EJECT=ejection; $I R=$ isovolumic relaxation; FILL=filling 

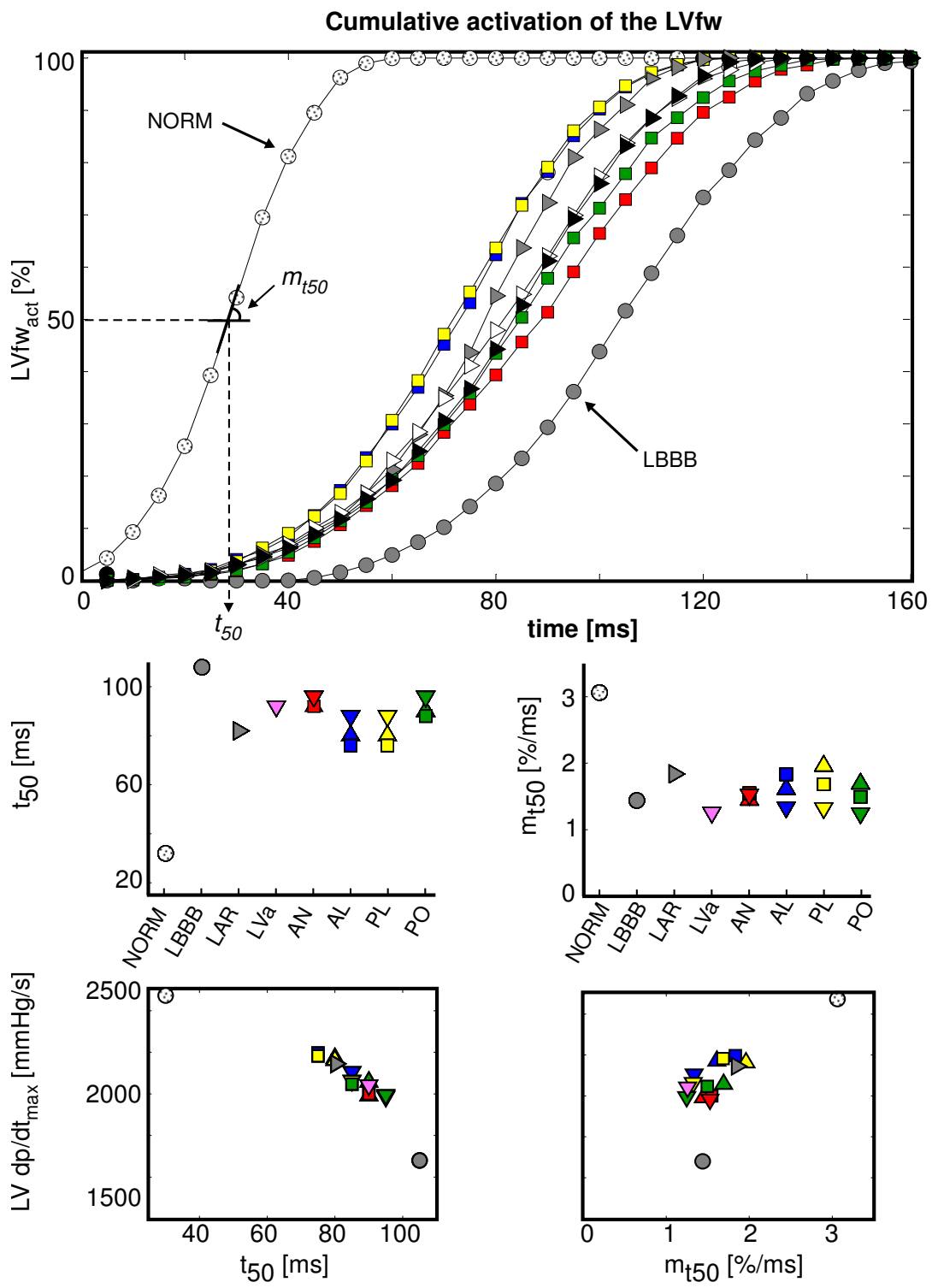

Figure 6.4: Activation patterns are characterized by the cumulative activation of the LVfw as a function of time. The top panel shows results of the NORM (dotted circles), $\angle B B B$ (grey circles), and a selection of the pacing simulations (non-circled symbols, see figure 6.2). The time needed to activate $50 \%$ of the LVfw tissue $\left(t_{50}\right)$ and the speed of activation recruitment at $t_{50}\left(m_{t 50}\right)$ are determined from these graphs. The middle panels show the results of $t_{50}$ (left) and $m_{t 50}$ (right) for all simulations. LAR=latest activated region; $\mathrm{LVa}=\mathrm{LV}$ apex; $\mathrm{AN}=$ anterior; $\mathrm{AL}=$ antero-lateral; $\mathrm{PL}=$ postero-lateral; $\mathrm{PO}=$ posterior. The bottom panels show the relation of both $t_{50}$ and $m_{t 50}$ with LV $d p / d t_{\max }$. 

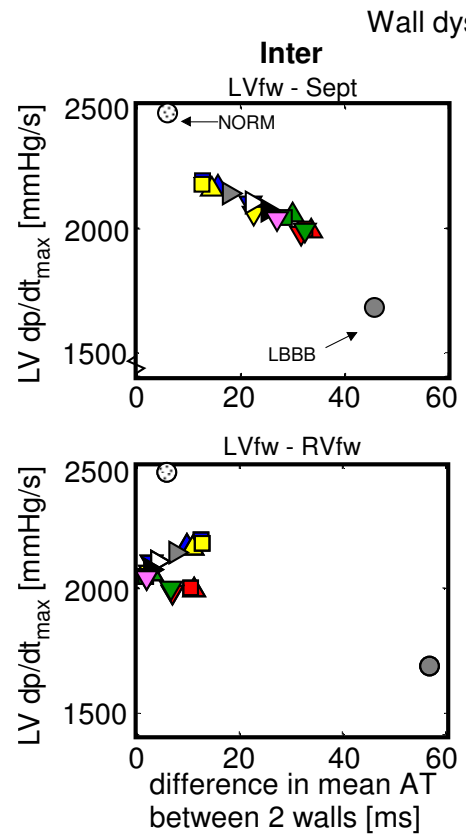
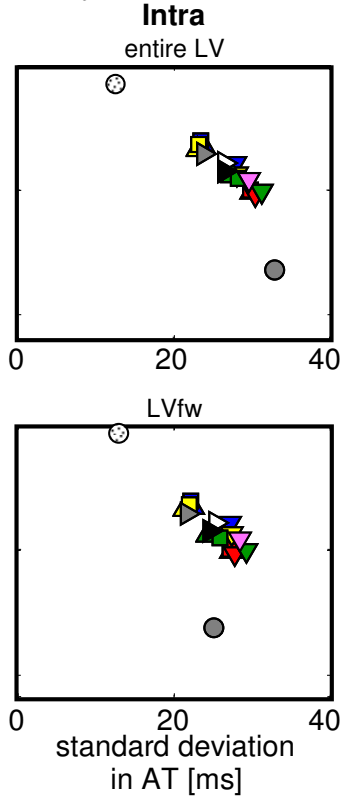

Figure 6.5: Relation between electrical dyssynchrony and LV $d p / d t_{\text {max }}$. Inter wall dyssynchrony (left) was determined between LVfw and septum (top), and LVfw and RVfw (bottom). Intra wall dyssynchrony (right) was determined in the entire LV (top), and the LVfw (bottom).

\subsection{Discussion}

In this study, we investigated the relation between LV electrode position during biventricular pacing and cardiac function in a finite element (FE) model of left and right ventricular electromechanics. The model replicated clinical observations such as the increase of $\mathrm{LV} d p / d t_{\max }$ and stroke work and the disappearance of a septal flash during $\mathrm{BiV}$ pacing. Acute hemodynamic response, expressed as increase in LV $d p / d t_{\max }$ and LV stroke work, was maximum when the LV electrode was positioned at the mid-basal lateral wall. Pacing from this region resulted in best resynchronization of the LV through high speed of activation recruitment, and consequently, low LV dyssynchrony. The LV pacing site that led to largest CRT response co-located with the latest activated region (LAR) for the simulation of $\angle B B B$, but not for the fascicular block simulations, suggesting that the association between LAR and optimal $\mathrm{LV}$ pacing site is based on coincidence rather than on electromechanical mechanisms.

Maximum CRT response by pacing the mid-level lateral wall The observation in our computational study that the fastest recruitment of LV activation and the largest hemodynamic effect was achieved by mid-basal LV lateral wall pacing, is corroborated by data from animal experiments [13] and patient studies [4]. The good performance of the mid-basal lateral wall as pacing site 


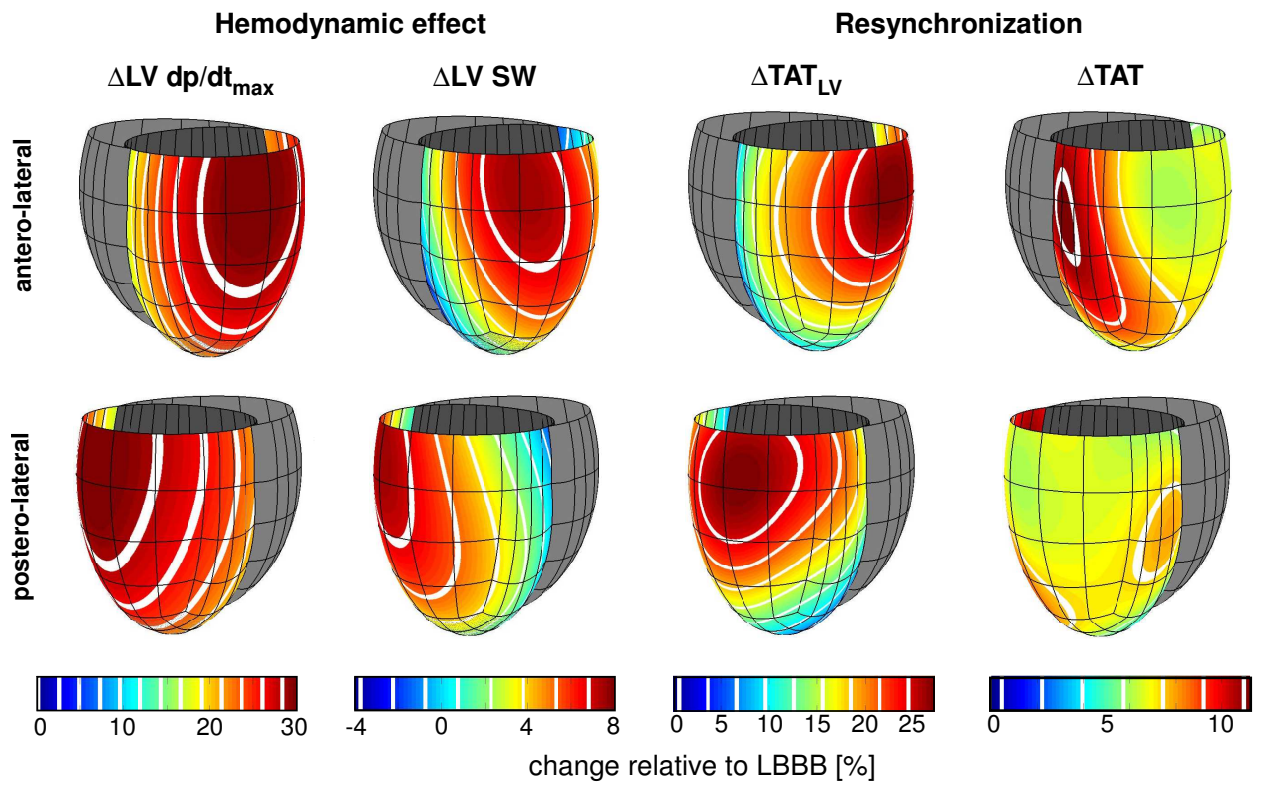

Figure 6.6: Functional epicardial maps showing the hemodynamic effect (change in LV $d p / d t_{\max }$ and LV stroke work), and resynchronization of the LV (decrease of $T A T_{l v}$ ), and of both ventricles (decrease of TAT) as function of the LV pacing site. Each set shows two orientations: antero-lateral (top) and postero-lateral (bottom). The color code reflects the change relative to $\angle B B B$. Baseline values: $\mathrm{LV} d p / d t_{\text {max }}=1680 \mathrm{mmHg} / \mathrm{s}, \mathrm{LV} \mathrm{SW}=0.92 \mathrm{~J}, \mathrm{TAT}=174 \mathrm{~ms}, T A T_{l v}=174 \mathrm{~ms}$. Note the different scales for each function variables.

may be understood easiest by considering that from this site the activation wavefront can move in all directions. In other words, the average distance to the pacing site is smaller for mid-basal LV lateral wall regions than for pure basal, often later activated, regions. Maximum CRT response through fast activation recruitment confirms suggestions from other computational studies $[22,14]$.

Role of anatomical location The relatively large area of the LV wall that can be paced to obtain a substantial hemodynamic response to CRT that we found, has also been corroborated by observations made in canine experiments [13]. This large region with at least reasonable hemodynamical effect might explain why larger clinical studies do not consistently find an effect of the anatomical region on (long-term) response to CRT [9].

Role of latest activated region Recent studies showed that response to CRT was better when baseline $A T$ in the LV pacing site was higher [12, 17, 34, 36]. The present study indicates that in case of typical LBBB the best resynchro- 

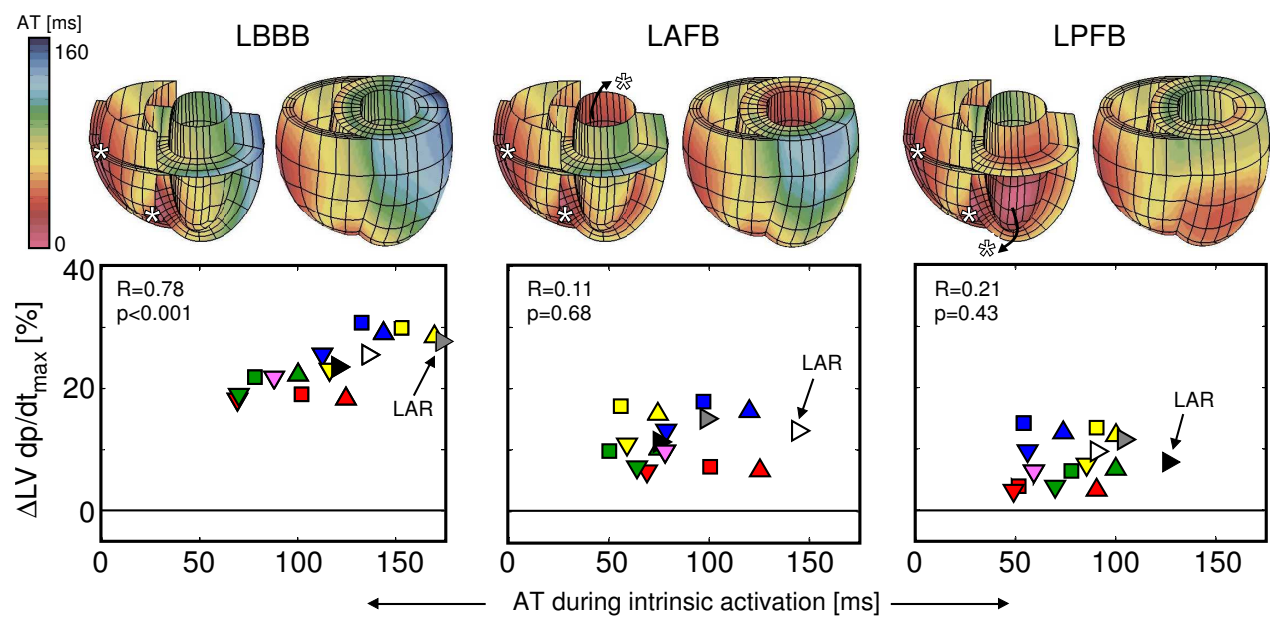

Figure 6.7: Relation between change in LV $d p / d t_{\max }$ and the activation delay of the LV pacing site during three baseline situations: $\angle B B B$ (left), $L A F B$ (middle), and $\angle P F B$ (right). The activation pattern of each baseline situation is shown on top. $\mathrm{LV} d p / d t_{\max }$ values (mean $\pm \mathrm{SD}$ ) were $1680 \pm 15,1863 \pm 72,1923 \pm 50 \mathrm{mmHg} / \mathrm{s}$, respectively.

nization is indeed achieved by pacing the RV in combination with pacing a site that is coincidentally close to the LAR. However, the LARs during fascicular block activation are located further away from the site that gave the best response. This discrepancy can be explained by the fact that in our simulations the ventricular activation during $\mathrm{BiV}$ pacing is determined solely by the activation wavefronts originating from the two pacing sites, and subsequently, baseline activation sequence becomes irrelevant. This is also the most commonly applied mode in clinical practice. Only in case of programming a relatively long AV-delay, intrinsic activation sequence may contribute to the activation sequence in paced heart beats, but this is not common and was therefore not investigated in our study.

The results of clinical studies that advocate the use of the LAR may, in part be explained by the fact that the delay at this location provides information whether this site is later activated than its adjacent regions, as well as that the entire LV is late activated, the latter implying that there is a substrate amenable for resynchronization. The importance of the latter is illustrated from the results in the simulations with fascicular blocks in our study. Altogether, our results indicate that the association between LAR and optimal LV pacing site is based on coincidence rather than on electromechanical mechanisms. 
Reduction of QRS duration It has been hypothesized that reduction of the QRS duration would predict a good response to CRT. However, response to CRT has also been observed in combination without reduction in QRS duration [23, 35] or in $T A T_{l v}$ [20]. These apparently conflicting data might be explained by the RV pacing-induced increase of electrical dyssynchrony in the RVfw while this ventricle was synchronously activated during intrinsic conduction. Very little attention has been paid to RV activation and function in CRT patients so far. The present study indicates that aiming at QRS reduction may even be disadvantageous, because maximum decrease of TAT was obtained when the LV pacing site was in anterior regions, i.e., regions that showed poor increase of hemodynamic response (figure 6.6).

Study assumptions and limitations The computational model is by definition a simplified representation of cardiovascular anatomy and physiology. For example, besides a distinction between endocardial and myocardial conduction velocities, no other regional differences in conduction were taken into account, nor dilated cardiomyopathy with decreased contractility. The two fascicular blocks are merely examples of non-typical LBBB conduction, used to illustrate the independence of the relation between baseline conduction pattern with corresponding location of the latest activated region and CRT response. Finally, ischemic cardiomyopathy was not taken into account.

Clinical implication The simulation results presented in this study give more insight into the possible mechanism for maximizing CRT response by choosing the LV pacing site. The present study provides several clues: 1) the anatomical position can be useful, especially aiming at mid-basal LV lateral wall, 2) measuring the delay in the latest activated region (LAR) can confirm the electrical substrate, but aiming at the LAR is not advisable, 3) aiming at maximum QRS reduction is not advisable. More research is needed to test if the generated hypotheses hold for the real patient.

Another implication of the present study would be that for optimal application of CRT it may be useful to determine, during CRT implantation, the site that provides maximal resynchronization of the LV wall and between LVfw and septum. Measurements such as echocardiographic measurement of LVfw and septal motion [23] or non-invasive ECG-imaging [15, 25] may be useful in this respect, although currently undoubtedly making the implantation procedure longer and more complicated. 


\subsection{Conclusion}

The present computational study provides novel insight in how maximum acute hemodynamic response by BiV pacing can be achieved. Most important factors are fast recruitment of LV activation, leading to maximum reduction in inter and intra LV dyssynchrony. This was achieved by pacing a central location on the LV lateral wall. Coincidentally, this region was near the LAR, but only in case of a typical LBBB activation.

\section{Acknowledgements}

This research was performed within the framework of CTMM, the Center for Translational Molecular Medicine (www.ctmm.nl), project COHFAR (grant 01C-203). Dr. Lumens received a grant within the framework of the Dr E. Dekker program of the Dutch Heart Foundation (NHS-2012T010). 


\section{References}

1. Abraham WT (2002). "Cardiac resynchronization therapy for heart failure: biventricular pacing and beyond." Curr Opin Cardiol 17.4, pp. 346-352.

2. Akar FG, Spragg DD, Tunin RS, Kass DA, and Tomaselli GF (2004). "Mechanisms underlying conduction slowing and arrhythmogenesis in nonischemic dilated cardiomyopathy." Circ Res 95.7, pp. 717-725.

3. Bovendeerd PHM, Kroon W, and Delhaas T (2009). “Determinants of left ventricular shear strain". Am J Physiol - Heart Circ Physiol 297.3, H1058-H1068.

4. Butter C, Auricchio A, Stellbrink C, Fleck E, Ding J, Yu Y, Huvelle E, Spinelli J, and Pacing Therapy for Chronic Heart Failure, Study Group (2001). "Effect of resynchronization therapy stimulation site on the systolic function of heart failure patients." Circulation 104.25, pp. 3026-3029.

5. Caldwell BJ, Trew ML, Sands GB, Hooks DA, LeGrice IJ, and Smaill BH (2009). “Three distinct directions of intramural activation reveal nonuniform side-to-side electrical coupling of ventricular myocytes." Circ Arrhythm Electrophysiol 2.4, pp. 433-440.

6. Cleland JGF, Daubert JC, Erdmann E, Freemantle N, Gras D, Kappenberger L, Tavazzi L, and C A R E-H F Study Investigators (2005). "The effect of cardiac resynchronization on morbidity and mortality in heart failure." N Engl J Med 352.15, pp. 1539-1549.

7. Durrer D, van Dam RT, Freud GE, Janse MJ, Meijler FL, and Arzbaecher RC (1970). "Total excitation of the isolated human heart." Circulation 41.6, pp. 899-912.

8. Fauchier L, Marie O, Casset-Senon D, Babuty D, Cosnay P, and Fauchier JP (2002). “Interventricular and intraventricular dyssynchrony in idiopathic dilated cardiomyopathy: a prognostic study with fourier phase analysis of radionuclide angioscintigraphy." J Am Coll Cardiol 40.11, pp. 2022-2030.

9. Foley PWX, Chalil S, Ratib K, Smith R, Prinzen F, Auricchio A, and Leyva F (2011). "Fluoroscopic left ventricular lead position and the long-term clinical outcome of cardiac resynchronization therapy." Pacing Clin Electrophysiol 34.7, pp. 785-797.

10. Fung JWH, Lam YY, Zhang Q, Yip GWK, Chan WWL, Chan GCP, Chan JYS, and Yu CM (2009). "Effect of left ventricular lead concordance to the delayed contraction segment on echocardiographic and clinical outcomes after cardiac resynchronization therapy." J Cardiovasc Electrophysiol 20.5, pp. 530-535.

11. Gasparini M, Mantica M, Galimberti P, Bocciolone M, Genovese L, Mangiavacchi M, Marchesina UL, Faletra F, Klersy C, Coates R, and Gronda E (2003). "Is the left ventricular lateral wall the best lead implantation site for cardiac resynchronization therapy?" Pacing Clin Electrophysiol 26.1 Pt 2, pp. 162-168.

12. Gold MR, Leman RB, Wold N, Sturdivant JL, and Yu Y (2014). "The effect of left ventricular electrical delay on the acute hemodynamic response with cardiac resynchronization therapy." J Cardiovasc Electrophysiol 25.6, pp. 624-630. 
13. Helm RH, Byrne M, Helm PA, Daya SK, Osman NF, Tunin R, Halperin HR, Berger RD, Kass DA, and Lardo AC (2007). "Three-dimensional mapping of optimal left ventricular pacing site for cardiac resynchronization." Circulation 115.8, pp. 953-961.

14. Huntjens PR, Walmsley J, Ploux S, Bordachar P, Prinzen FW, Delhaas T, and Lumens $\mathrm{J}$ (2014). "Influence of left ventricular lead position relative to scar location on response to cardiac resynchronization therapy: a model study." Europace 16, pp. 62-68.

15. Jia P, Ramanathan C, Ghanem RN, Ryu K, Varma N, and Rudy Y (2006). “Electrocardiographic imaging of cardiac resynchronization therapy in heart failure: observation of variable electrophysiologic responses." Heart Rhythm 3.3, pp. 296-310.

16. Kerckhoffs RCP, Bovendeerd PHM, Kotte JCS, Prinzen FW, Smits K, and Arts T (2003). "Homogeneity of cardiac contraction despite physiological asynchrony of depolarization: a model study." Ann Biomed Eng 31.5, pp. 536-547.

17. Khan FZ, Virdee MS, Palmer CR, Pugh PJ, O'Halloran D, Elsik M, Read PA, Begley D, Fynn SP, and Dutka DP (2012). “Targeted left ventricular lead placement to guide cardiac resynchronization therapy: the TARGET study: a randomized, controlled trial." J Am Coll Cardiol 59.17, pp. 1509-1518.

18. Kronborg MB, Albertsen AE, Nielsen JC, and Mortensen PT (2009). "Long-term clinical outcome and left ventricular lead position in cardiac resynchronization therapy." Europace 11.9, pp. 1177-1182.

19. Kroon W, Delhaas T, Arts T, and Bovendeerd P (2009). “Computational analysis of the myocardial structure: Adaptation of myofiber orientations through deformation in three dimensions". Med Imag Anal 13, pp. 346-353.

20. Lumens J, Ploux S, Strik M, Gorcsan 3rd J, Cochet H, Derval N, Strom M, Ramanathan C, Ritter P, Haïssaguerre M, Jaïs P, Arts T, Delhaas T, Prinzen FW, and Bordachar P (2013). “Comparative electromechanical and hemodynamic effects of left ventricular and biventricular pacing in dyssynchronous heart failure: electrical resynchronization versus left-right ventricular interaction." J Am Coll Cardiol 62, pp. 2395-2403.

21. Merchant FM, Heist EK, McCarty D, Kumar P, Das S, Blendea D, Ellinor PT, Mela T, Picard MH, Ruskin JN, and Singh JP (2010). "Impact of segmental left ventricle lead position on cardiac resynchronization therapy outcomes." Heart Rhythm 7.5, pp. 639-644.

22. Niederer SA, Lamata P, Plank G, Chinchapatnam P, Ginks M, Rhode K, Rinaldi CA, Razavi R, and Smith NP (2012). "Analyses of the redistribution of work following cardiac resynchronisation therapy in a patient specific model." PLoS One 7.8, e43504.

23. Pitzalis MV, Iacoviello M, Romito R, Massari F, Rizzon B, Luzzi G, Guida P, Andriani A, Mastropasqua F, and Rizzon P (2002). "Cardiac resynchronization therapy tailored by echocardiographic evaluation of ventricular asynchrony." J Am Coll Cardiol 40.9, pp. 1615-1622.

24. Ploux S, Lumens J, Whinnett Z, Montaudon M, Strom M, Ramanathan C, Derval N, Zemmoura A, Denis A, De Guillebon M, et al. (2013). “Noninvasive electrocardiographic mapping to improve patient selection for cardiac resynchronization therapy: beyond QRS duration and left bundle branch block morphology." J Am Coll Cardiol 61.24, pp. 2435-2443.

25. Ramanathan C, Jia P, Ghanem R, Ryu K, and Rudy Y (2006). "Activation and repolarization of the normal human heart under complete physiological conditions." Proc Natl Acad Sci U S A 103.16, pp. 6309-6314.

26. Rawling DA, Joyner RW, and Overholt ED (1985). "Variations in the functional electrical coupling between the subendocardial Purkinje and ventricular layers of the canine left ventricle." Circ Res 57.2, pp. 252-261.

27. Rossillo A, Verma A, Saad EB, Corrado A, Gasparini G, Marrouche NF, Golshayan AR, McCurdy R, Bhargava M, Khaykin Y, et al. (2004). "Impact of coronary sinus lead position on biventricular pacing: mortality and echocardiographic evaluation during long-term followup." J Cardiovasc Electrophysiol 15.10, pp. 1120-1125. 
28. Saxon LA, Olshansky B, Volosin K, Steinberg JS, Lee BK, Tomassoni G, Guarnieri T, Rao A, Yong P, Galle E, Leigh J, Ecklund F, and Bristow MR (2009). "Influence of left ventricular lead location on outcomes in the COMPANION study." J Cardiovasc Electrophysiol 20.7, pp. 764768.

29. Singh JP, Klein HU, Huang DT, Reek S, Kuniss M, Quesada A, Barsheshet A, Cannom D, Goldenberg I, McNitt S, Daubert JP, Zareba W, and Moss AJ (2011). "Left ventricular lead position and clinical outcome in the multicenter automatic defibrillator implantation trial-cardiac resynchronization therapy (MADIT-CRT) trial." Circulation 123.11, pp. 1159-1166.

30. Strik M, Rademakers LM, van Deursen CJM, van Hunnik A, Kuiper M, Klersy C, Auricchio A, and Prinzen FW (2012). "Endocardial left ventricular pacing improves cardiac resynchronization therapy in chronic asynchronous infarction and heart failure models." Circ Arrhythm Electrophysiol 5.1, pp. 191-200.

31. Strik M, van Deursen CJM, van Middendorp LB, van Hunnik A, Kuiper M, Auricchio A, and Prinzen FW (2013). "Transseptal conduction as an important determinant for cardiac resynchronization therapy, as revealed by extensive electrical mapping in the dyssynchronous canine heart." Circ Arrhythm Electrophysiol 6.4, pp. 682-689.

32. Thébault C, Donal E, Meunier C, Gervais R, Gerritse B, Gold MR, Abraham WT, Linde C, Daubert JC, and R.E.V.E.R.S.E. Study Group (2012). "Sites of left and right ventricular lead implantation and response to cardiac resynchronization therapy observations from the REVERSE trial." Eur Heart J 33.21, pp. 2662-2671.

33. Tracy CM, Epstein AE, Darbar D, Dimarco JP, Dunbar SB, Estes 3rd NAM, Ferguson Jr TB, Hammill SC, Karasik PE, Link MS, et al. (2012). “2012 ACCF/AHA/HRS Focused Update of the 2008 Guidelines for Device-Based Therapy of Cardiac Rhythm Abnormalities: a report of the American College of Cardiology Foundation/American Heart Association Task Force on Practice Guidelines." Heart Rhythm 9.10, pp. 1737-1753.

34. Ypenburg C, van Bommel RJ, Delgado V, Mollema SA, Bleeker GB, Boersma E, Schalij MJ, and Bax JJ (2008). "Optimal left ventricular lead position predicts reverse remodeling and survival after cardiac resynchronization therapy." J Am Coll Cardiol 52.17, pp. 1402-1409.

35. Yu CM, Fung JWH, Chan CK, Chan YS, Zhang Q, Lin H, Yip GWK, Kum LCC, Kong SL, Zhang Y, and Sanderson JE (2004). "Comparison of efficacy of reverse remodeling and clinical improvement for relatively narrow and wide QRS complexes after cardiac resynchronization therapy for heart failure." J Cardiovasc Electrophysiol 15.9, pp. 1058-1065.

36. Zanon F, Baracca E, Pastore G, Fraccaro C, Roncon L, Aggio S, Noventa F, Mazza A, and Prinzen F (2014). "Determination of the longest intrapatient left ventricular electrical delay may predict acute hemodynamic improvement in patients after cardiac resynchronization therapy." Circ Arrhythm Electrophysiol 7.3, pp. 377-383. 
Chapter 7

General Discussion 


\subsection{Introduction}

This thesis aims to contribute to the procedure of model-assisted diagnosis and treatment selection in cardiac disease. To this end, a generic finite element model of left and right ventricular electromechanics has been developed. As input for some model parameters, such as myofiber orientation, was not available from measurement data with sufficient accuracy, we tested whether input settings for myofiber orientation could be determined through adaptation [2]. Myofiber orientation was adapted in response to mechanical load as suggested by Kroon et al. [33]. Adaptive reorientation of myofibers was found to predict myofiber orientations that were within the range of experimental data. The adaptive myofiber reorientation improved cardiac function in a simplified geometry of the left ventricle (LV) as well as in the more realistic geometry of both LV and right ventricle (RV). Simulation of Cardiac Resynchronization Therapy (CRT) in the biventricular model with adapted myofiber orientation showed that maximum resynchronization led to maximum response and this was achieved by pacing the LV lateral wall.

\subsection{Adaptative reorientation as a tool to estimate my- ofiber orientation}

\section{Main Achievements}

The uniqueness and functionality of the specific myofiber orientation patterns in the heart has been widely accepted [22, 42,52]. The potential of computational models to assist in cardiac disease and the need for a detailed description of the myofiber orientation in these models have been recognized too. A strategy often followed to personalize myofiber orientation in patient-specific geometries, is to map an atlas of a human myofiber orientation pattern onto these geometries [10,48]. Such an atlas provides the average and variability of the myofiber orientation pattern within a population [37]. It is unclear how the mapping procedure affects model outcome, because the specific combination of geometry and myofiber orientation might be important too. A large sensitivity of cardiac function to a variation in myofiber orientation within the anatomical range as reported in [37] has already been shown from studies on computational models of the LV $[6,20,33]$. As there is no reason to assume that sensitivity is lower in BiV models, we did not perform an extended sensitivity analysis.

The hypothesis for the existence of a feedback mechanism from mechanical load to a change in myofiber orientation has been deduced from experiments that showed absence of significant heterogeneity in metabolism and 
myofiber shortening throughout the wall $[38,57]$. Though load-induced reorientation of myofibers is not corroborated by experimental data [19, 25, 60], this does not exclude the existence of this mechanism, considering the limited accuracy of techniques for measuring myofiber orientation [18]. In addition, the promising result of predicting a realistic myofiber orientation together with a decrease in heterogeneity of local mechanical function in [33] motivated us to continue this work.

The choice for the initial myofiber orientation throughout the thesis was based on knowledge from experimental [22, 39, 42, 52] and computational data [45] (chapters 2-5). After reorientation in the normal left ventricle (LV), a pattern for the transmural component in myofiber orientation was found that remained qualitatively similar irrespective of a variation in the initial myofiber orientation field (chapter 2), a variation of boundary conditions (chapter 2 ), a change in myocardial tissue properties (chapter 4), and a variation of geometry (chapter 5). Although the rate of change of myofiber orientation reduced as adaptation progressed, suggesting convergence to a steady-state solution, such a solution was not reached, so quantitative comparison between simulations remained problematic.

A large research area is focussed on improving techniques for measuring the myocardial structure [21,51,54], but a significant step in improving the accuracy has yet to be made. The limited accuracy of techniques for measuring myofiber orientation also limits a critical evaluation of the model predicted myofiber orientation. As it turned out that shear deformation is sensitive to the choice of especially the transmural component in myofiber orientation [6], we used the comparison between model computed and experimentally measured shear strains as a more critical test to evaluate model results. In both the LV and BiV model, agreement between model computed and experimentally measured temporal variations of circumferential-radial shear improved after shear-induced myofiber reorientation (chapters 2, 4, 5). In the SIT LV, the agreement only improved for the torsion data (chapter 3 ). In a first analysis of the results from the BiV model in chapter 5, qualitative differences in torsion were observed between the LV-only and the BiV model. Further investigation is needed to find out where the differences originated from.

\section{Limitations}

Discrepancies between model computed and experimental data and the absence of a steady-state solution for myofiber orientation may result from limitations in the models of mechanics and adaptation. Most important simplifications will be addressed below. 
Model of cardiac mechanics Boundary conditions were applied at the base of the ventricles to account for the interaction of the model with structures left out of the model, e.g., annulus fibrosus, atria, and great vessels. Throughout this thesis we applied simple kinematic boundary conditions. The errors related to this simplification of the true interaction reduce with increasing distance from the basal plane, and are acceptable for assessment of deformation at the mid-level myocardium. During adaptation, however, unphysiological mechanics in the basal region affect myofiber reorientation in the basal region. The unphysiological reoriented myofiber orientation will affect mechanics in the sub-basal region, and subsequently, reorientation of myofiber in the subbasal region. Eventually, regions further away from the base become affected too (chapter 2). For realistic results of adaptation, these boundary conditions need to be reconsidered.

Throughout this thesis we assumed the passive material to behave transversely isotropic [6, 27]. Experimental data showed that the myocardium behaves orthotropic, which is explained from the organization of myocytes into sheets [14, 47]. Sheets are predominantly oriented in transmural direction, and presumably facilitate thickening of the wall during ejection [36, 43]. It has been suggested that the sheet-direction coincides with the principal shear direction. As there are two such directions, oriented at $\pm 45^{\circ}$ to the principal strain direction, and sheet orientation could occur in both these directions $[3,11,24]$, a transversely isotropic behavior seems plausible. Even if the orientation could be measured, ascribing different material properties requires complex three-dimensional testing [14].

Model of cardiac adaptation We only accounted for myofiber reorientation, while other adaptation mechanisms are known as well. Clinically, one of the most evident examples of adaptation is the global change in ventricular wall mass (hypertrophy) and cavity volume (dilation) in response to changes in hemodynamic loading. In addition, ventricular wall mass can even change locally $[58,59]$. Considering the asymmetric hypertrophy as observed in ventricular paced canine hearts in [58,59], growth might for example be important to take into account when predicting the long-term response to CRT.

In a preliminary study, a model of cardiac growth was developed that simulated growth based on the principle of a local stimulus-response relation [15]. Based on the model in [34], growth was simulated with an updated reference configuration to account for the effect of tissue turnover. Local active work density was used as local mechanical stimulus to predict cardiac growth. This growth stimulus combines myofiber stress and strain, which both have been proven to influence growth [7, 23, 49]. This growth model could predict asymmetric ventricular growth in an asynchronously activated 

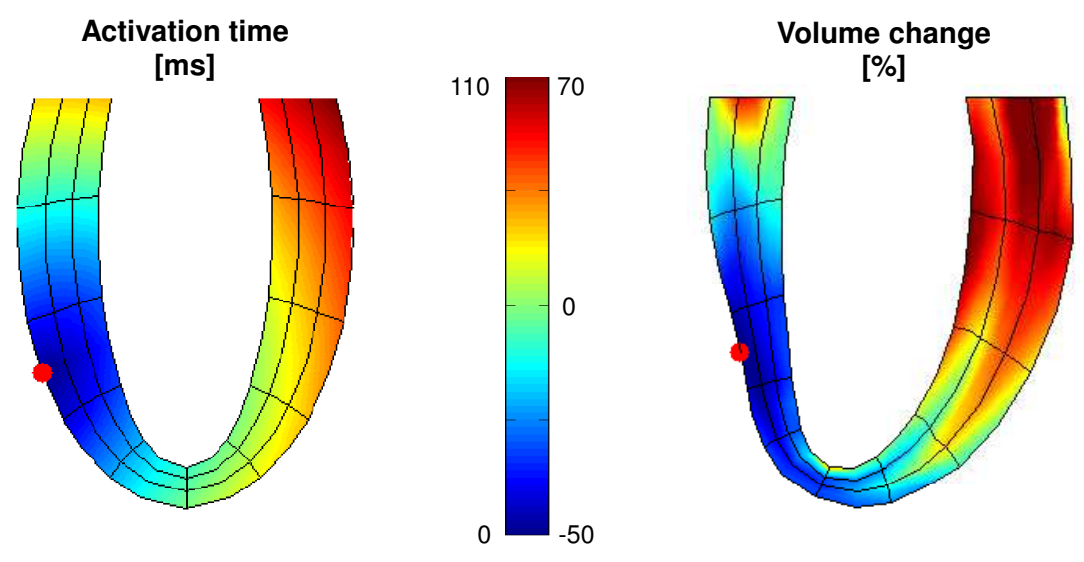

Figure 7.1: Left: Long-axis cross-section of the initial LV, with the sequence of activation as induced by positioning a pacing lead on the septal side of the LV. Right: Longaxis cross-section of the grown LV with the spatial distribution of volume change. The growth stimulus active work density induced shrinkage of early activated and growth of late activated regions. Figure adapted from [15].

LV (figure 7.1). After 14 growth increments, early-activated regions near the pacing site decreased up to $40 \%$, whereas late-activated regions increased up to $70 \%$ of the initial volumes. These preliminary model predictions are promising considering the maximum volume change of $40 \%$ as observed in canine experiments $[58,59]$ and motivate further investigation of this growth model.

\subsection{Towards clinical application of computational mod- els}

\section{Main achievements}

Computational models of both left and right ventricular electromechanics are needed to assist in clinical decision-making in cardiac disease. The first biventricular finite element models were published about a decade ago $[28,56]$. Meanwhile, several BiV FE models have been presented [1, 29, 40, 46, 48, 61]. However, only a few of them showed to be capable of simulating consecutive cardiac cycles $[29,46]$. Because proper evaluation of the results should be done in steady-state, simulating consecutive cardiac cycles is indispensable. In this thesis, we have successfully developed a BiV FE model that was capable of simulating multiple consecutive cardiac cycles. The stability of the simulations was found to depend critically on proper meshing [17] and myofiber orientation (chapter 5). Simulation of one cardiac cycle took about 2 
hours on a single core of an i7 processor, which makes the model feasible for clinical application.

Computational models of biventricular electromechanics can be used to guide hypothesis generation and to test hypotheses for complicated clinical problems. As these models are by definition a simplified representation of cardiovascular physiology, experiments need to be designed to confirm or reject the generated hypotheses. An example of hypothesis testing was presented in chapter 6 where the model was deployed to investigate how maximum response to CRT could be obtained by varying the position of the LV pacing site. Maximum resynchronization of the LV led to maximum increase of cardiac function, and this was obtained by pacing from the mid-level LV lateral wall. A coincidentally strong correlation was found between delay at the pacing site measured during typical left bundle branch block (LBBB) activation and increase of cardiac function. The non-causality of the relation between baseline activation pattern and improvement of cardiac function during biventricular pacing was directly clear from the model setup. Despite the fact that this model setup best mimicked the current understanding of the physiology involved, the non-causality turned out not to be obvious from a clinical point of view. Therefore, such clarifying insights illustrate the added value of computational studies.

\section{Limitations}

To investigate the influence of various imposed electrical activation patterns on pump function in chapter $\mathbf{6}$, the model in chapter 5 was extended with the Eikonal model to compute electrical wave propagation. This model was preferred over the bidomain model because of computational efficiency. In addition, solutions of the Eikonal and bidomain model were reported to match closely [9]. Indeed, model computed normal and left bundle branch block activation patterns matched closely with experimental results $[16,53]$. As the Eikonal model only solves for the arrival time of activation, pathologies at the cellular level, e.g. ion channel pathologies, cannot be simulated with this model. In addition, no electro-mechanical coupling was taken into account. For these purposes, more extended computational models are needed [10, 41, 55].

Asynchronous electrical activation led to changes in local mechanics: earlyactivated regions shorten at low stress, because late-activated regions lengthen passively. Consequently, heterogeneity in local work increases. It has been suggested that local mechanics might induce adaptation of electrophysiology [26]. For modeling long-term effects of pacing, adaptation of electrophysiology through mechanical-electrical feedback might be important to take into account as well [35].

The case of a heart with only LBBB, to which the BIV model was applied 
in chapter 6, represents an idealized situation when compared to heart of a patient visiting the clinician. Patients often have dilated and ischemic cardiomyopathy. Previous studies reported a disproportionate worse outcome when the pacing site was in ischemic myocardium compared to any other pacing site in non-ischemic myocardium $[4,5,12,13,50]$. The question is which LV pacing site gives maximum response to CRT in the presence of ischemia.

In a preliminary study, we investigated the relation between LV pacing site and response to CRT in ischemic cardiomyopathy. Therefore, we simulated two of the more common locations of infarction, i.e., due to occlusion of the left anterior descending artery (LADi) and of the left circumflex artery (LCXi). Ischemic tissue was modeled to have $90 \%$ reduction of conduction velocities, 15 fold increase of passive stiffness, and no active stress development $[30,60]$. Infarct sizes were $16 \%$ for LADi and $21 \%$ for LCXi of the LV wall volume. An infarct borderzone was defined for the transition from ischemic to healthy tissue. Similar to chapter 6 , cardiac function was investigated during normal activation sequence, LBBB, and CRT with different LV pacing site (figure 7.2). In both models of ischemia, biventricular pacing improved LV $d p / d t_{\max }$ compared to intrinsic activation $[8,44]$. In the LADi heart, a maximum response of about $55 \%$ was obtained by pacing the LV lateral wall. In the LCXi heart, however, maximum response was only $28 \%$ and differences in response between the different LV pacing sites were small. Pacing from the antero-lateral wall did not result in better response than pacing from clear anterior or posterior regions. Similar to chapter 6, decrease of LV dyssynchrony was strongly correlated with increase of LV $d p / d t_{\max }$ (see figure 6.5). These preliminary results indicate that gain in resynchronization is reduced for LCXi, but is little or not reduced for LADi.

\subsection{Future perspective}

This thesis contains a small, though important step in the procedure to construct a patient-specific model that can be used to assist in clinical decision making. The first steps have been made to test the feasibility of the model to simulate pathology-specific cases. The fact that a converged solution is obtained, while having large spatial heterogeneities in myocardial tissue properties and mechanical loading indicates that the model is robust and has great potential for clinical application.

A nice example of the work-flow from figure 1.1 for constructing a patientspecific model of cardiac electromechanics has been presented in [48]. Data from magnetic resonance imaging, electrophysiology catheters, and pressure catheters were used to set all the parameter values in a biventricular model of electromechanics. After solving the inverse problem, a match in model pre- 
A
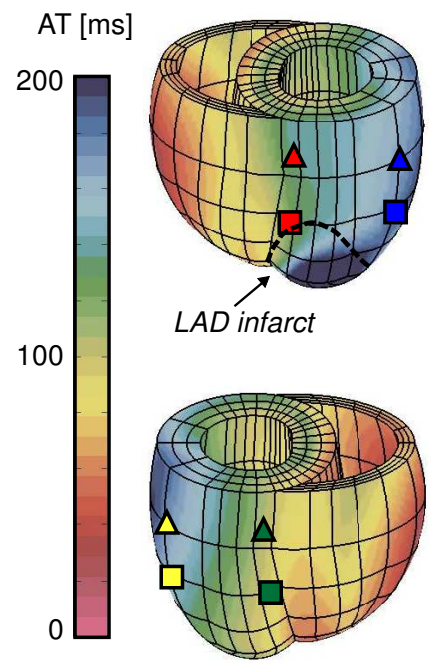

B

Figure 7.2: Response to CRT in the ischemic heart. A: Sequence of activation during LBBB with ischemia due to occlusion of the left anterior descending artery (LADi, left column), and due to occlusion of the left circumflex artery (LCXi, right column). Colored symbols indicate the position of the LV electrode. B: Relation between electrical dyssynchrony and LV $d p / d t_{\max }$. See figure 6.5 for comparison of results without ischemia.

LADi

Intra LV

dyssynchrony
LCXi
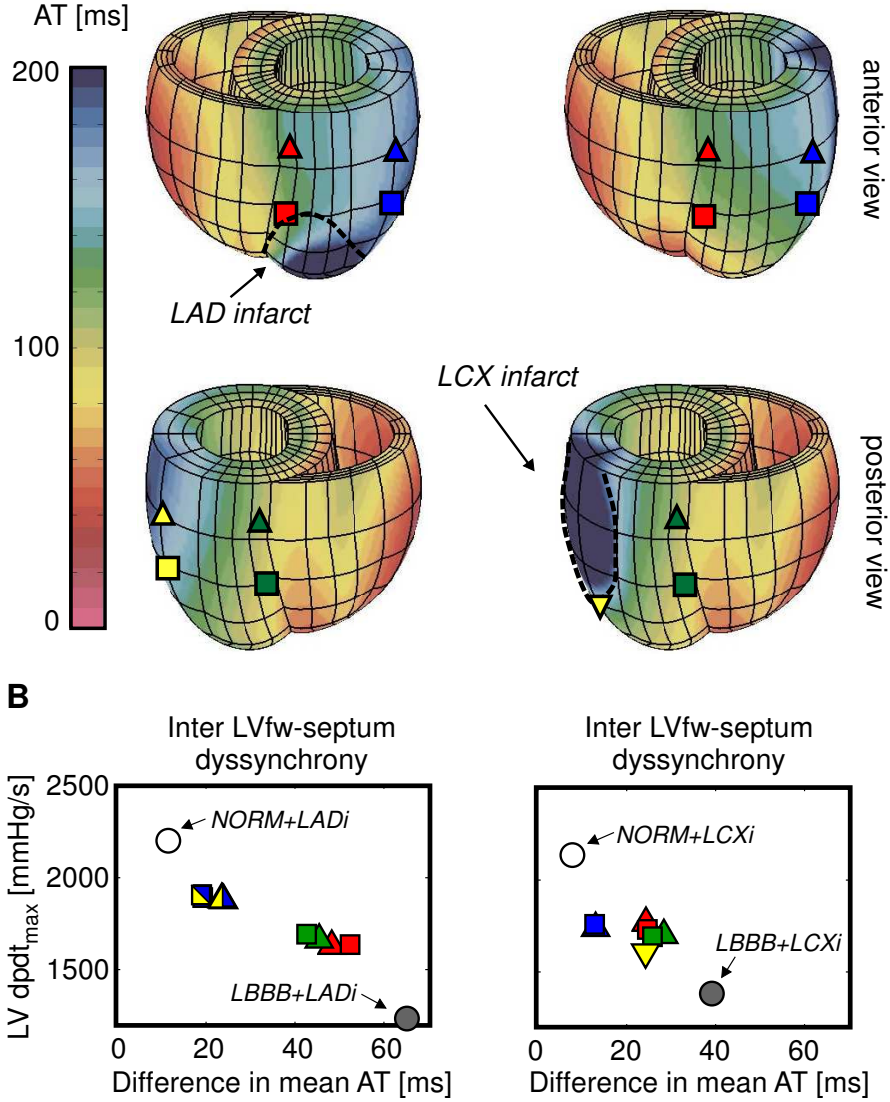

Inter LVfw-septum dyssynchrony
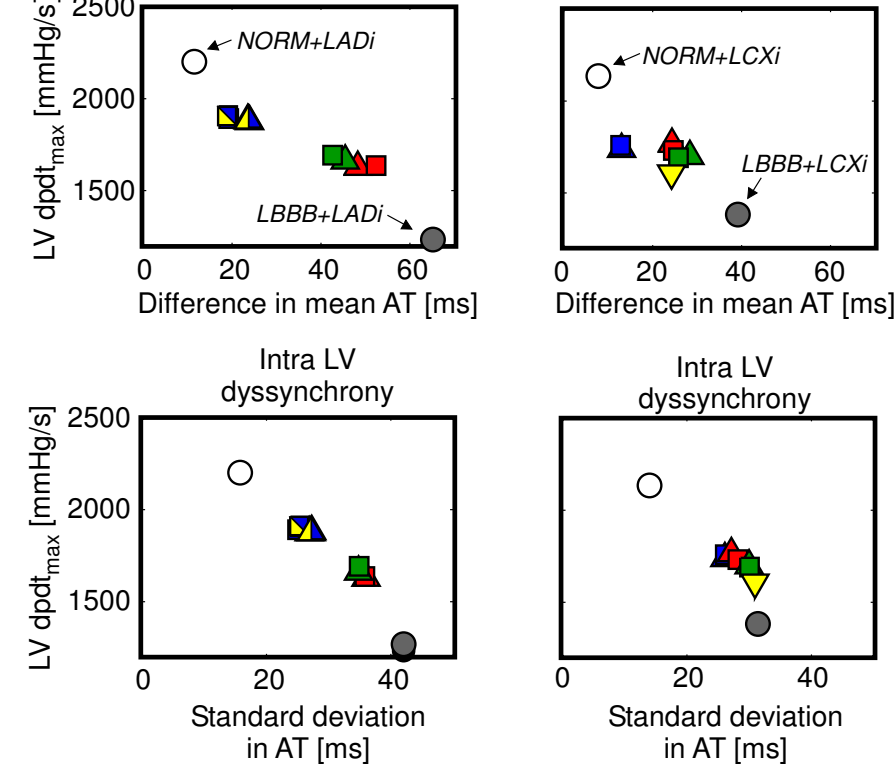

Intra LV dyssynchrony

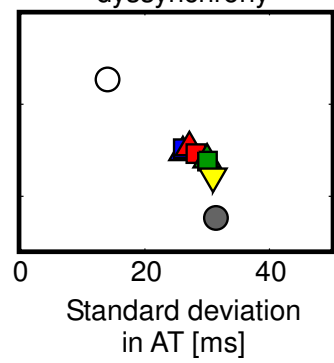


dicted and measured LV $d p / d t_{\max }$ was obtained with a mean error less than $5 \%$. In addition, the computed error between computed and measured radial and circumferential strain was about $2 \mathrm{~mm}$. As was shown in the model of chapter 4, these model outputs varied little at several different model input settings. Therefore, it is recommendable to evaluate the model output with a more critical test to avoid the risk for non-uniqueness of the solution. In addition, we recommend to investigate how sensitive the model output is for variations in myofiber orientation of about 5 to $10^{\circ}$. Considering these difficulties, the construction of a patient-specific model might be far ahead. Still, biventricular models of electromechanics seem to offer great potential to assist in clinical decision making, though the next step might address pathologyspecific rather than patient-specific questions [10, 30, 31, 32, 41].

\subsection{General Conclusions}

In this thesis we developed a computational model of left and right ventricular electromechanics. Myofiber orientation was determined from a submodel, describing adaptive reorientation of myofibers in response to local tissue strain. The pattern for the transmural component in myofiber orientation obtained from the model agreed with experimental observations and was associated with better pump function than in absence of adaptive myofiber orientation. The sensitivity of cardiac pump function to small changes in myofiber orientation advocate the need for techniques that can measure in vivo patient-specific myofiber orientation with high accuracy. In absence of accurate patient-specific information, myofiber reorientation seems a useful approach to estimate myofiber orientation. The developed model with adaptive myofiber orientation was able to increase mechanistic insight in the relation between the site of LV pacing and hemodynamic response in Cardiac Resynchronization Therapy (CRT). Maximum response to CRT was obtained during maximal resynchronization of the LV, which is achieved by pacing at the LV lateral wall. 


\section{References}

1. Aguado-Sierra J, Krishnamurthy A, Villongco C, Chuang J, Howard E, Gonzales MJ, Omens J, Krummen DE, Narayan S, Kerckhoffs RCP, and McCulloch AD (2011). "Patientspecific modeling of dyssynchronous heart failure: a case study." Prog Biophys Mol Biol 107.1, pp. 147-155.

2. Arts T, Bovendeerd P, Delhaas T, and Prinzen F (2003). “Modeling the relation between cardiac pump function and myofiber mechanics". J Biomech 36, pp. 731-736.

3. Arts T, Costa KD, Covell JW, and McCulloch AD (2001). "Relating myocardial laminar architecture to shear strain and muscle fiber orientation." Am J Physiol Heart Circ Physiol 280.5, $\mathrm{H} 2222-\mathrm{H} 2229$.

4. Bilchick KC, Kuruvilla S, Hamirani YS, Ramachandran R, Clarke SA, Parker KM, Stukenborg GJ, Mason P, Ferguson JD, Moorman JR, et al. (2014). “Impact of mechanical activation, scar, and electrical timing on cardiac resynchronization therapy response and clinical outcomes." J Am Coll Cardiol 63.16, pp. 1657-1666.

5. Bleeker GB, Kaandorp TAM, Lamb HJ, Boersma E, Steendijk P, de Roos A, van der Wall EE, Schalij MJ, and Bax JJ (2006). "Effect of posterolateral scar tissue on clinical and echocardiographic improvement after cardiac resynchronization therapy." Circulation 113.7, pp. 969976.

6. Bovendeerd PHM, Kroon W, and Delhaas T (2009). “Determinants of left ventricular shear strain". Am J Physiol - Heart Circ Physiol 297.3, H1058-H1068.

7. Bupha-Intr T, Holmes JW, and Janssen PML (2007). "Induction of hypertrophy in vitro by mechanical loading in adult rabbit myocardium." Am J Physiol Heart Circ Physiol 293.6, H3759-H3767.

8. Chalil S, Foley PWX, Muyhaldeen SA, Patel KCR, Yousef ZR, Smith REA, Frenneaux $\mathrm{MP}$, and Leyva F (2007). "Late gadolinium enhancement-cardiovascular magnetic resonance as a predictor of response to cardiac resynchronization therapy in patients with ischaemic cardiomyopathy." Europace 9.11, pp. 1031-1037.

9. Colli Franzone P and Guerri L (1993). "Spread of excitation in 3-D models of the anisotropic cardiac tissue. I. Validation of the eikonal model". Math Biosci 113, pp. 145-209.

10. Constantino J, Hu Y, and Trayanova NA (2012). "A computational approach to understanding the cardiac electromechanical activation sequence in the normal and failing heart, with translation to the clinical practice of CRT.". Prog Biophys Mol Biol 110.2-3, pp. 372-379.

11. Costa KD, Takayama Y, McCulloch AD, and Covell JW (1999). "Laminar fiber architecture and three-dimensional systolic mechanics in canine ventricular myocardium". Am J Physiol - Heart Circ Physiol 276, pp. 595-607.

12. de Roest GJ, Wu L, de Cock CC, Hendriks ML, Delnoy PPHM, van Rossum AC, and Allaart CP (2014). "Scar tissue-guided left ventricular lead placement for cardiac resynchronization therapy in patients with ischemic cardiomyopathy: an acute pressure-volume loop 
study." Am Heart J 167.4, pp. 537-545.

13. Delgado V, Bommel RJ van, Bertini M, Borleffs CJW, Marsan NA, Arnold CT, Nucifora G, Veire NRL van de, Ypenburg C, Boersma E, Holman ER, Schalij MJ, and Bax JJ (2011). "Relative merits of left ventricular dyssynchrony, left ventricular lead position, and myocardial scar to predict long-term survival of ischemic heart failure patients undergoing cardiac resynchronization therapy." Circulation 123.1, pp. 70-78.

14. Dokos S, Smaill BH, Young AA, and LeGrice IJ (2002). "Shear properties of passive ventricular myocardium". Am J Physiol 283, H2650-H2659.

15. Donk L van der (2014). "Modelling cardiac growth driven by local myofibre work density". MA thesis. Eindhoven University of Technology.

16. Durrer D, van Dam RT, Freud GE, Janse MJ, Meijler FL, and Arzbaecher RC (1970). "Total excitation of the isolated human heart." Circulation 41.6, pp. 899-912.

17. Flores de la Parra A (2012). "A finite element model of biventricular cardiac mechanics". MA thesis. Eindhoven University of Technology.

18. Geerts-Ossevoort L (2002). "Cardiac myofiber reorientation: a mechanism for adaptation?" PhD thesis. Eindhoven University of Technology.

19. Geerts-Ossevoort L, Bovendeerd P, Prinzen F, Arts T, and Nicolay K (2001). "Myofiber orientation in the normal and infarcted heart, assessed with MR-diffusion tensor imaging". In: Computers in Cardiology 2001, pp. 621-624.

20. Geerts-Ossevoort L, Kerckhoffs R, Bovendeerd P, and Arts T (2003). "Towards Patient Specific Models of Cardiac Mechanics: A Sensitivity Study". In: Functional Imaging and Modeling of the Heart. Ed. by I Magnin, J Montagnat, P Clarysse, J Nenonen, and T Katila. Vol. 2674. Lecture Notes in Computer Science. Springer Berlin Heidelberg, pp. 81-90.

21. Gilbert SH, Benoist D, Benson AP, White E, Tanner SF, Holden AV, Dobrzynski H, Bernus O, and Radjenovic A (2012). "Visualization and quantification of whole rat heart laminar structure using high-spatial resolution contrast-enhanced MRI.". Am J Physiol Heart Circ Physiol 302.1, H287-H298.

22. Grimm A, Katele K, and Lin H (1976). "Fiber bundle direction in the mammalian heart. An extension of the 'nested shells' model." Basic Res. Cardiol. 71, pp. 381-388.

23. Grossman W, Jones D, and McLaurin LP (1975). "Wall stress and patterns of hypertrophy in the human left ventricle." J Clin Invest 56.1, pp. 56-64.

24. Helm PA, Tseng HJ, Younes L, McVeigh ER, and Winslow RL (2005). "Ex vivo 3D diffusion tensor imaging and quantification of cardiac laminar structure." Magn Reson Med 54.4, pp. 850-859.

25. Helm PA, Younes L, Beg MF, Ennis DB, Leclercq C, Faris OP, McVeigh E, Kass D, Miller MI, and Winslow RL (2006). "Evidence of structural remodeling in the dyssynchronous failing heart." Circ Res 98.1, pp. 125-132.

26. Jeyaraj D, Wilson LD, Zhong J, Flask C, Saffitz JE, Deschênes I, Yu X, and Rosenbaum DS (2007). "Mechanoelectrical feedback as novel mechanism of cardiac electrical remodeling." Circulation 115.25, pp. 3145-3155.

27. Kerckhoffs RCP, Bovendeerd PHM, Kotte JCS, Prinzen FW, Smits K, and Arts T (2003a). "Homogeneity of cardiac contraction despite physiological asynchrony of depolarization: a model study." Ann Biomed Eng 31.5, pp. 536-547.

28. Kerckhoffs RCP, Bovendeerd PHM, Prinzen FW, Smits K, and Arts T (2003b). "Intraand interventricular asynchrony of electromechanics in the ventricularly paced heart". J Eng Math 47, pp. 201-216.

29. Kerckhoffs RCP, Healy SN, Usyk TP, and D MA (2006). "Computational Methods for Cardiac Electromechanics". J PROC IEEE 94.4, pp. 769-783.

30. Kerckhoffs RCP, McCulloch AD, Omens JH, and Mulligan LJ (2009). "Effects of biventricular pacing and scar size in a computational model of the failing heart with left bundle branch block". Med Im Anal 13, pp. 362-369. 
31. Kerckhoffs RCP, Omens JH, McCulloch AD, and Mulligan LJ (2010). “Ventricular dilation and electrical dyssynchrony synergistically increase regional mechanical nonuniformity but not mechanical dyssynchrony". Circ Heart Fail 3, pp. 528-536.

32. Kerckhoffs RC, Omens JH, and McCulloch AD (2012). "Mechanical discoordination increases continuously after the onset of left bundle branch block despite constant electrical dyssynchrony in a computational model of cardiac electromechanics and growth". Europace 14, pp. v65-v72.

33. Kroon W, Delhaas T, Arts T, and Bovendeerd P (2009a). "Computational analysis of the myocardial structure: Adaptation of myofiber orientations through deformation in three dimensions". Med Imag Anal 13, pp. 346-353.

34. Kroon W, Delhaas T, Arts T, and Bovendeerd PHM (2009b). "Computational modeling of volumetric soft tissue growth: application to the cardiac left ventricle". Biomechanics and Modeling in Mechanobiology 8(4), pp. 301-309.

35. Kuijpers NHL, Eikelder tHMM, Bovendeerd PHM, Verheule S, Arts T, and Hilbers PAJ (2008). "Mechanoelectric feedback as a trigger mechanism for cardiac electrical remodeling: A model study". Ann Biomed Eng 36, pp. 1816-1835.

36. LeGrice IJ, Smaill BH, Chai LZ, Edgar SG, Gavin JB, and Hunter PJ (1995). “Laminar structure of the heart: ventricular myocyte arrangement and connective tissue architecture in the dog." Am J Physiol 269.2 Pt 2, H571-H582.

37. Lombaert H, Peyrat JM, Croisille P, Rapacchi S, Fanton L, Cheriet F, Clarysse P, Magnin I, Delingette H, and Ayache N (2012). "Human atlas of the cardiac fiber architecture: study on a healthy population." IEEE Trans Med Imaging 31.7, pp. 1436-1447.

38. MacGowan GA, Shapiro EP, Azhari H, Siu CO, Hees PS, Hutchins GM, Weiss JL, and Rademakers FE (1997). "Noninvasive measurement of shortening in the fiber and cross-fiber directions in the normal human left ventricle and in idiopathic dilated cardiomyopathy". Circulation 96, pp. 535-541.

39. Matsumura H, Aizawa Y, and Kumaki K (1990). "Myocardial architecture in situs inversus vicerum totalis". In: Developmental cardiology: morphogenesis and function. Ed. by E Clark and A Takao. ISBN-13: 978-0-879-93382-1. Futura Pub Co, Mount Kisco, pp. 605-624.

40. Niederer SA, Plank G, Chinchapatnam P, Ginks M, Lamata P, Rhode KS, Rinaldi CA, Razavi R, and Smith NP (2011). "Length-dependent tension in the failing heart and the efficacy of cardiac resynchronization therapy". Cardiovascular Research 89(2), pp. 336-343.

41. Niederer SA, Lamata P, Plank G, Chinchapatnam P, Ginks M, Rhode K, Rinaldi CA, Razavi R, and Smith NP (2012). "Analyses of the redistribution of work following cardiac resynchronisation therapy in a patient specific model." PLoS One 7.8, e43504.

42. Nielsen PM, LeGrice IJ, Smaill BH, and Hunter PJ (1991). "Mathematical model of geometry and fibrous structure of the heart". Am J Physiol - Heart Circ Physiol 260, H1365-H1378.

43. Omens JH, Usyk TP, Li Z, and McCulloch AD (2002). "Muscle LIM protein deficiency leads to alterations in passive ventricular mechanics." Am J Physiol Heart Circ Physiol 282.2, H680-H687.

44. Rademakers LM, van Kerckhoven R, van Deursen CJM, Strik M, van Hunnik A, Kuiper M, Lampert A, Klersy C, Leyva F, Auricchio A, Maessen JG, and Prinzen FW (2010). "Myocardial infarction does not preclude electrical and hemodynamic benefits of cardiac resynchronization therapy in dyssynchronous canine hearts." Circ Arrhythm Electrophysiol 3.4, pp. 361368.

45. Rijcken JM, Bovendeerd PHM, Schoofs AJG, Van Campen DH, and Arts T (1999). “Optimization of cardiac fiber orientation for homogeneous fiber strain during ejection". Ann Biomed Eng 27, pp. 289-297.

46. Saint-Marie J, Chapelle D, Cimrman R, and Sorine M (2006). "Modeling and estimation of the cardiac electromechanical activity". Comp and Struc 84, pp. 1743-1759. 
47. Schmid H, O'Callaghan P, Nash MP, Lin W, LeGrice IJ, Smaill BH, Young AA, and Hunter PJ (2008). "Myocardial material parameter estimation: a non-homogeneous finite element study from simple shear tests." Biomech Model Mechanobiol 7.3, pp. 161-173.

48. Sermesant M, Chabiniok R, Chinchapatnam P, Mansi T, Billet F, Moireau P, Peyrat JM, Wong K, Relan J, Rhode K, et al. (2012). "Patient-specific electromechanical models of the heart for the prediction of pacing acute effects in CRT: a preliminary clinical validation." Med Image Anal 16.1, pp. 201-215.

49. Simpson DG, Majeski M, Borg TK, and Terracio L (1999). "Regulation of cardiac myocyte protein turnover and myofibrillar structure in vitro by specific directions of stretch." Circ Res 85.10, e59-e69.

50. Spragg DD, Dong J, Fetics BJ, Helm R, Marine JE, Cheng A, Henrikson CA, Kass DA, and Berger RD (2010). “Optimal left ventricular endocardial pacing sites for cardiac resynchronization therapy in patients with ischemic cardiomyopathy." J Am Coll Cardiol 56.10, pp. 774-781.

51. Stephenson RS, Boyett MR, Hart G, Nikolaidou T, Cai X, Corno AF, Alphonso N, Jeffery N, and Jarvis JC (2012). “Contrast enhanced micro-computed tomography resolves the 3-dimensional morphology of the cardiac conduction system in mammalian hearts." PLoS One 7.4, e35299.

52. Streeter Jr D, Spotnitz HM, Patel DP, Ross Jr J, and Sonnenblick EH (1969). "Fiber orientation in the canine left ventricle during diastole and systole." Circ Res 24.3, pp. 339-347.

53. Strik M, van Middendorp LB, and Vernooy K (2012). "Animal models of dyssynchrony." J Cardiovasc Transl Res 5.2, pp. 135-145.

54. Toussaint N, Stoeck CT, Schaeffter T, Kozerke S, Sermesant M, and Batchelor PG (2013). "In vivo human cardiac fibre architecture estimation using shape-based diffusion tensor processing." Med Image Anal.

55. Trayanova NA, Constantino J, and Gurev V (2010). "Models of stretch-activated ventricular arrhythmias." J Electrocardiol 43.6, pp. 479-485.

56. Usyk TP, LeGrice IJ, and McCulloch AD (2002). "Computational model three dimensional cardiac electromechanics". Comp Visual Sci 4, pp. 249-257.

57. van der Vusse GJ, Arts T, Glatz JF, and Reneman RS (1990). "Transmural differences in energy metabolism of the left ventricular myocardium: fact or fiction." J Mol Cell Cardiol 22.1, pp. 23-37.

58. van Oosterhout MF, Prinzen FW, Arts T, Schreuder JJ, Vanagt WY, Cleutjens JP, and Reneman RS (1998). "Asynchronous electrical activation induces asymmetrical hypertrophy of the left ventricular wall." Circulation 98.6, pp. 588-595.

59. Vernooy K, Verbeek XAAM, Peschar M, Crijns HJGM, Arts T, Cornelussen RNM, and Prinzen FW (2005). "Left bundle branch block induces ventricular remodelling and functional septal hypoperfusion." Eur Heart J 26.1, pp. 91-98.

60. Walker JC, Ratcliffe MB, Zhang P, Wallace AW, Fata B, Hsu EW, Saloner D, and Guccione JM (2005). "MRI-based finite-element analysis of left ventricular aneurysm." Am J Physiol Heart Circ Physiol 289.2, H692-H700.

61. Xia L, Huo M, Wei Q, Liu F, and Crozier S (2005). "Analysis of cardiac ventricular wall motion based on a three-dimensional electromechanical biventricular model." Phys Med Biol 50.8, pp. 1901-1917. 



\section{Summary}

Clinical diagnosis of cardiac pathologies and treatment selection is based on statistical interpretation of data measured on the patient and using the experience and knowledge of a cardiologist. In fact, it results in a best diagnosis and treatment for an average patient. Ideally, the diagnosis and treatment selection are tailored to the patient at hand, so that success of treatment can be guaranteed. To this end, direct assessment of the myocardial tissue properties would be needed, because clinical measurements can only give indirect information about the state of the heart. However, such direct assessment would be highly invasive and a time-consuming task.

Mathematical models could assist in tailored clinical diagnosis and treatment selection by providing spatial maps of tissue properties of a particular patient. In an iterative process, model parameters have to be adjusted to obtain maximum agreement between model predicted and clinically observed cardiac function. The obtained maps of tissue properties could be used as an extra source of information by the cardiologist to arrive at a patient-specific diagnosis and treatment selection. The aim of this thesis is to contribute to the procedure of model-assisted diagnosis and treatment selection. To this purpose, a mathematical model of biventricular (BiV) electromechanics was developed and applied to a clinically relevant case.

The inverse analysis for setting the model parameters, however, introduces a risk for non-uniqueness of the solution that may be decreased by increasing the number of clinical observations (chapter 1). Unfortunately, methods to assess relevant information, e.g. myofiber orientation, are limited in accuracy. An alternative approach to overcome such limitations is to use knowledge on cardiac adaptation. Therefore, in the model development, we specifically focussed on determination of myofiber orientation through adaptation of myofiber orientation in response to mechanical load.

This thesis builds upon an existing model of shear-induced myofiber reorientation. We started with a simpler model of the left ventricle (LV) only, to investigate the long-term effect of initial conditions and boundary conditions at the base of the LV on myofiber reorientation (chapter 2). The choice of boundary conditions influenced long-term development of myofiber ori- 
entation while the effect of initial setting of myofiber orientation disappeared. Besides directly comparing model-predicted and measured myofiber orientations, model results were evaluated by comparing model-computed and measured cardiac deformations. This provides a more critical test, because measurement data on myofiber orientation lack sufficient accuracy. Adaptive myofiber reorientation reduced differences between model-predicted and experimental observations of deformation.

Besides the normal LV, we also consider the LV in a mirror-imaged position of organs, i.e. Situs Inversus Totalis (SIT). The SIT LV has quite an abnormal myofiber orientation pattern with respect to the normal LV. As the SIT LV consists of the same type of cells as the normal LV, a similar stimulus-response relation is expected. In chapter 3 , we used the SIT LV as an extreme test case to explore whether myofiber orientations of the SIT LV can also be predicted by shear-induced myofiber reorientation. Indeed, agreement improved after myofiber reorientation between model-predicted and measured data on deformation in the SIT LV.

Although myofiber reorientation improved agreement between computed and measured deformation patterns during a cardiac cycle, a complete match was not obtained. Therefore, we investigated in chapter 4 whether the remaining differences were caused by limitations in the LV mechanics model. By extending the model with a physiological sequence of activation and active stress development in not only fiber but also cross-fiber direction, computed deformation patters were found to become more physiologic.

To assist in clinical decision-making, mathematical models need to contain both a right and left ventricle. Therefore, a $\mathrm{BiV}$ model of cardiac mechanics was presented in chapter 5 . The sensitivity of cardiac function to geometry and myofiber orientation was investigated. The results showed that a small average change in myofiber orientation of about $8^{\circ}$ already led to a significant increase in cardiac pump work of about $18 \%$. These findings indicate the importance of a thorough effort to address a realistic myofiber orientation. The model of myofiber reorientation seemed a useful approach to estimate myofiber orientation in absence of accurate patient-specific information.

In chapter 6, the model was applied to the clinical case of cardiac resynchronization therapy (CRT). This therapy aims to resynchronize abnormal activation between the right and left ventricle, and subsequently myocardial contraction to improve pump function in heart failure patients. Therefore, the ventricles are artificially stimulated by a lead positioned in the right ventricle and a lead on the LV free wall. The model with adapted myofiber orientation of chapter $\mathbf{5}$ was combined with a model for electrical wave propagation to investigate the relation between LV lead position and cardiac function. The results showed that improvement of cardiac pump function was maximal by pacing a central location on the LV lateral wall. 
In conclusion, this thesis contributed to the procedure of model-assisted diagnosis and treatment selection by developing a mathematical model of right and left ventricular electromechanics. Model-computed cardiac function turned out to be sensitive for the choice of myofiber orientation within the reported range of experimental data. The sub-model of mechanicallyinduced adaptation of myofiber orientation seemed a useful approach to estimate patient-specific myofiber orientation in absence of accurate experimental data. The developed model increased mechanistic insight in the relation between the site of LV pacing and the hemodynamic response in CRT. 



\section{Samenvatting}

Het klinisch diagnosticeren van een hartziekte en de keuze voor een bijpassende behandeling berusten op statistische interpretatie van data gemeten bij de patiënt, waarbij de cardioloog gebruik maakt van zijn kennis en ervaring. Het resultaat van deze procedure is eigenlijk een beste diagnose en behandeling die afgestemd is op de gemiddelde patiënt. Idealiter zouden de diagnose en behandeling toegespitst worden op de patiënt in kwestie, zodat het succes van de behandeling gegarandeerd kan worden. Hiertoe zouden de weefseleigenschappen van het hart direct gemeten moeten worden, aangezien klinische metingen slechts indirecte informatie over de weefseleigenschappen geven. Het is echter een onbegonnen zaak om weefseleigenschappen direct te meten.

Wiskundige modellen kunnen de procedure voor patiënt-specifiek diagnosticeren en behandelkeuze ondersteunen door de weefseleigenschappen van de betreffende patiënt in kaart te brengen. Dit gebeurt tijdens een iteratief proces, waarbij modelparameters worden aangepast totdat de verschillen tussen cardiale functie zoals voorspeld door het model en zoals gemeten bij de patiënt zo klein mogelijk zijn. De voorspelde weefseleigenschappen kunnen vervolgens dienen als een extra bron van informatie voor de cardioloog bij het stellen van de patiënt-specifieke diagnose en behandelkeuze. Het doel van dit proefschrift is dan ook het bijdragen aan de procedure van modelgestuurde diagnose en behandelkeuze. Om dit doel te bereiken is een wiskundig model van de elektromechanische eigenschappen van het hart ontwikkeld en toegepast op een klinisch relevante situatie.

Het instellen van modelparameters via de inverse analyse gaat echter gepaard met het risico dat er geen unieke oplossing bestaat. Dit risico kan verkleind worden wanneer het aantal klinische observaties toeneemt (hoofdstuk 1). Helaas kan echter niet alle relevante informatie, zoals spiervezeloriëntatie, met voldoende nauwkeurigheid gemeten worden. Een andere manier om toch over deze informatie te kunnen beschikken, is gebruik te maken van kennis over cardiale adaptatie. In de ontwikkeling van het model is dan ook speciaal aandacht besteed aan het bepalen van de spiervezeloriëntatie via adaptatie van de oriëntatie als gevolg van mechanische belasting. 
Dit proefschrift bouwt voort op een bestaand model voor heroriëntatie van spiervezels als gevolg van afschuiving van de spiervezel ten opzichte van het omliggende weefsel. In hoofdstuk 2 wordt een simpeler model van alleen de linker ventrikel (LV) gebruikt om uit te zoeken hoe de heroriëntatie op lange termijn wordt beïnvloed door de keuze van de beginconditie van de vezeloriëtatie en de keuze van randvoorwaarden aan de basis van de LV. Hieruit is gebleken dat de heroriëntatie wel wordt beïnvloed door de keuze van de randvoorwaarden, maar niet door de keuze van de beginconditie van de spiervezeloriëntatie. Naast het één-op-één vergelijken van de modelvoorspelde en experimenteel gemeten spiervezeloriëntatie, is ook de modelvoorspelde cardiale deformatie vergeleken met gemeten deformatie. Dit laatste wordt gezien als een kritische test voor de evaluatie van het model, omdat de spiervezeloriëntatie momenteel nog niet met voldoende nauwkeurigheid gemeten kan worden. Na heroriëntatie van spiervezels kwamen modelvoorspelde en gemeten cardiale deformatie beter overeen.

Naast de normale LV, wordt ook de LV in situs inversus totalis (SIT) beschouwd. Bij deze aandoening is de positie van de organen links-rechts gespiegeld. De SIT LV heeft een essentieel andere spiervezeloriëntatie vergeleken met de normale LV. Omdat de SIT LV wel uit hetzelfde type cellen bestaat als in de normale LV, is een vergelijkbare stimulus-respons relatie te verwachten. In hoofdstuk 3 is de SIT LV gebruikt als extreme proef om uit te zoeken of spiervezeloriëntatie ook in de SIT LV voorspeld kon worden door het algoritme voor heroriëntatie. Ook in de SIT LV leidde adaptieve heroriëntatie van de spiervezels tot een betere overeenstemming van modelvoorspelde en gemeten deformatie.

Hoewel na heroriëntatie van de spiervezels de overeenstemming tussen berekende en gemeten deformatiepatronen verbeterde, bleven er verschillen bestaan. In hoofdstuk 4 is daarom onderzocht of de overgebleven verschillen worden veroorzaakt door beperkingen in het LV mechanicamodel. Hiertoe werd het model uitgebreid met een fysiologische opeenvolging van activatie en met de ontwikkeling van actieve spanning in niet alleen de spiervezelrichting maar ook in de richtingen loodrecht hierop. Met deze toevoegingen werden de overeenkomsten tussen modelvoorspelde en gemeten deformatiepatronen weer een stukje beter.

Voor het gebruik van wiskundige modellen ter ondersteuning van de klinische besluitvorming, dienen deze modellen te bestaan uit zowel een LV als een rechter ventrikel (RV). In hoofdstuk 5 wordt het biventriculaire (BiV) mechanicamodel gepresenteerd. In dit hoofdstuk is onderzocht hoe gevoelig de berekende cardiale functie was voor geometrie en spiervezeloriëntatie. De resultaten lieten zien dat een gemiddelde verandering in vezeloriëntatie van slechts $8^{\circ}$ leidde tot een toename van $18 \%$ in de pompfunctie van het hart. Deze bevinding geeft aan hoe belangrijk is het om spiervezeloriëntatie met 
hoge nauwkeurigheid te kunnen meten. Het model voor spiervezelheroriëntatie lijkt een nuttig alternatief te zijn voor het schatten van spiervezeloriëntatie bij afwezigheid van voldoende nauwkeurige patiënt-specifieke informatie.

Hoofdstuk 6 beschrijft de toepassing van het model voor de klinische situatie tijdens cardiale resynchronizatie therapie (CRT). Deze therapie heeft als doel abnormale ventriculaire impulsgeleiding bij patiënten met hartfalen te corrigeren, zodat de pompfunctie verbetert. De ventrikels worden kunstmatig gestimuleerd door een elektrode in de RV en een elektrode op de vrije wand van de LV. Om dit te simuleren is het model met geadapteerde spiervezeloriëntatie uit hoofdstuk 5 gecombineerd met een model voor elektrische impuls geleiding. Dit gecombineerde model is gebruikt om de relatie tussen positie van de LV elektrode en cardiale functie te onderzoeken. Hieruit bleek dat de toename van LV pompfunctie maximaal was wanneer de LV gestimuleerd werd vanaf een centrale positie op de LV laterale wand.

Tot slot kan er geconcludeerd worden dat het onderzoek zoals gepresenteerd in dit proefschrift bijdraagt aan de procedure voor modelgestuurde diagnose en behandelkeuze door het ontwikkelen van een wiskundig model van RV en LV elektromechanica (hoofdstuk 7). De modelvoorspelde cardiale functie bleek erg gevoelig voor de keuze van spiervezeloriëntatie binnen de gerapporteerde spreiding op experimentele data. Het algoritme voor mechanischgestuurde adaptatie van spiervezeloriëntatie bleek een nuttige, alternatieve methode voor het afschatten van patiënt-specifieke vezeloriëntatie bij afwezigheid van nauwkeurige experimentele data. Met behulp van het ontwikkelde model werd mechanistisch inzicht verkregen in de relatie tussen de locatie van stimulatie op de vrije wand van de LV en de verandering van hemodynamische functie tijdens CRT. 



\section{Valorisatie}

\section{Inleiding}

Het hart is de pomp in onze bloedsomloop en zorgt ervoor dat het bloed onophoudelijk door ons lichaam stroomt. Het ritme waarin het hart pompt wordt geregeld door een groepje cellen (sinusknoop) in één van de vier kamers van het hart, de rechter boezem. Deze cellen geven zo'n 60 à 70 keer per minuut een elektrische puls af. Deze puls verspreidt zich via snel geleidende cellen over de hele hartspier waarna de spiercellen samentrekken.

In een gezond hart trekken de rechter en linker kamer nagenoeg tegelijkertijd samen. Het synchroon samentrekken van de hartspier zorgt voor een efficiënte pompfunctie. In patiënten met hartfalen is deze pompfunctie verslechterd en in ongeveer $25 \%$ van de gevallen wordt dit veroorzaakt doordat de gecoördineerde samentrekking verstoord is. Vaak hebben deze patiënten een blokkade in het snelle geleidingssysteem, waardoor de ene kamer eerder samentrekt dan de andere. Dit wordt ook wel dyssynchroon hartfalen (DHF) genoemd.

Patiënten met dyssynchroon hartfalen worden behandeld met cardiale resynchronizatie therapie (CRT). Bij deze therapie wordt een pacemaker geplaatst met een elektrode in zowel de rechter als linker kamer (biventriculair). De elektrodes geven tegelijkertijd een prikkel zodat de kamers weer synchroon samentrekken.

$\mathrm{Al}$ aan het eind van de jaren '90 werd bekend dat biventriculair pacen de pompfunctie van patiënten met DHF verbetert. De eerste implantaties bij mensen werden rond de eeuwwisseling verricht en sindsdien is het aantal implantaties alleen maar gestegen. Momenteel worden er in Nederland jaarlijks zo'n 600 pacemakers per 1 miljoen inwoners geïmplanteerd. Dit aantal ligt rond het Europese gemiddelde aantal implantaties per miljoen inwoners. In landen als Duitsland en België worden maar liefst twee keer zo veel implantaties verricht. 


\section{Relevantie en doelgroepen}

Na circa 15 jaar van implantaties is er uit verschillende klinische studies veel informatie beschikbaar gekomen over de effectiviteit van CRT. Uit resultaten van meerdere klinische trials blijkt dat bij zeker $30 \%$ van de patiënten het implanteren van een CRT device niet of nauwelijks leidt tot een verbetering van de pompfunctie. Dit wijst erop dat de onderliggende ziekte die het hartfalen veroorzaakt nog niet voldoende begrepen wordt. Om het slagingspercentage van CRT te verhogen, dient meer kennis verkregen te worden van het (dys)functioneren van het hart. Deze kennis is soms moeilijk te verkrijgen vanuit de kliniek, omdat niet alle weefseleigenschappen tot in het kleinste detail gemeten kunnen worden.

Zoals beschreven in dit proefschrift, kunnen computermodellen assisteren bij het bepalen van de pathologie. Deze modellen bevatten namelijk de weefseleigenschappen die anders lastig te meten zijn. Via de voorwaartse relatie die is beschreven met fysische wetten, kan vanuit de weefseleigenschappen een voorspelling worden gedaan van de functie van het hart. Op deze manier kan de kennis en het inzicht verkregen worden die nodig is voor het stellen van de juiste dianose. Dit is in eerste instantie relevant voor artsen. Zij hebben naast de gangbare anamnese en hun ervaring een extra bron van informatie ter beschikking.

Naast het bepalen van de pathologie, kunnen modellen ook het effect van een ingreep simuleren om te bepalen welke behandeling het best geschikt zou zijn voor de betreffende patiënt. Hoofdstuk 6 van dit proefschrift toont hier een voorbeeld van. Hierin laten we namelijk zien dat de mate waarin de pompfunctie toeneemt, afhangt van de locatie van stimulatie op de vrije wand van de linker kamer. Het kiezen van de optimale plek voor maximale toename van pompfunctie is één van de strategieën om het slagingspercentage voor response op CRT te vergroten, wat zal leiden tot beter welzijn van de patiënt. Daarnaast kan patiënten een ingrijpende interventie bespaard blijven wanneer zij in de voorspelling geen baat zullen hebben bij de therapie. Vanuit nieuwe kennis en inzichten kunnen deze patiënten op een andere manier geholpen worden.

Tot slot draagt modelgestuurde diagnose bij aan een efficiëntere werkwijze in de gezondheidszorg wat leidt tot een reductie van de kosten in het ziekenhuis. Deze kostenreductie vertaalt zich door naar de maatschappij. Zorgverzekeraars kunnen zorg tegen scherpere prijzen bij de ziekenhuizen inkopen, waardoor de premie omlaag kan. 


\section{Activiteiten en Producten}

Dit onderzoek beschrijft de eerste stappen naar de ontwikkeling van softwarepakketten voor klinisch gebruik. Het beschreven model in dit proefschrift kan worden doorontwikkeld tot een gebruiksvriendelijke tool voor artsen. De arts heeft via de anamnese en aanvullend onderzoek enkele kenmerken van het hart van de patiënt gemeten. Deze data dient vervolgens als input voor het model, zodat het model patiënt-specifiek wordt gemaakt. Vervolgens wordt door het simuleren van het effect van verschillende locaties van de elektrodes op de pompfunctie bepaald wat de optimale plaats van elektrische stimulatie is voor deze patiënt. Deze informatie wordt meegenomen in de planning van de interventie.

In de toekomst zal mogelijk elk ziekenhuis zijn uitgerust met een ingenieursbureau waar met deze software wordt gewerkt. De technici van het ingenieursbureau construeren met de input data van de arts het patiënt-specifieke model. De voorspellingen van het model worden teruggekoppeld naar de arts, waarop de arts een patiënt-specifieke diagnose en behandelkeuze formuleert.

\section{Planning en Realisatie}

Om het gebruik van computermodellen voor klinisch gebruik te realiseren, zal het huidige model eerst verder ontwikkeld moeten worden. In dit proefschrift is slechts een generiek model gepresenteerd. Een volgend PhD-traject zou zich kunnen richten op het patiënt-specifiek maken van het model. Het model biedt namelijk de mogelijkheid om globale patiënt-specifieke verschillen zoals, wandvolumes, kamerafmetingen, en spatiële variatie in weefseleigenschappen mee te geven. De eerste stappen naar het patiënt-specifiek maken van het model kunnen bestaan uit het indelen van patiënten in groepen met of zonder ischemische gebieden, of in groepen met een bepaalde mate van geleidingsstoornis. Patiënten met ischemie kunnen weer verder onderverdeeld worden in plaats en grootte van het ischemische gebied. De resultaten van het model kunnen dan geëvalueerd worden met de karakteristieke data per patiëntengroep.

De volgende stap is het gebruiksvriendelijk maken van de software. Hierbij kunnen bedrijven worden ingeschakeld die geïnteresseerd zijn in het aanbieden van deze software. Bedrijven als Pie Medical of Philips kunnen hier interesse voor hebben. Daarnaast zouden pacemakerfabrikanten als Medtronic, St. Jude Medical of Boston Scientific geïnteresseerd kunnen zijn in deze software om zo de ontwikkelingen in hun afzetmarkt onder controle te houden. Via deze software kunnen namelijk nieuwe ideeën ontstaan voor nieuwe type 
pacemakers en wellicht wordt er inzicht verkregen in welke types pacemakers op den duur van de markt zullen verdwijnen. Deze informatie is van belang bij het bepalen van de strategieën van pacemakerfabrikanten. 


\section{Dankwoord}

De afgelopen vier jaar zijn voorbij gevlogen. Van net afgestudeerde tot zelfstandig onderzoeker, met alle ups en downs die bij een PhD-traject horen. De ups zijn toch wel het meeste bijgebleven. Het mag dan wel zo zijn dat mijn naam de voorkant van dit proefschrift staat, maar dit proefschrift was nooit tot stand gekomen zonder hulp, inzet en kennis van vele anderen in welke vorm dan ook. Daarom sluit ik dit werk af met een woord van dank.

Allereerst wil ik mijn promotoren, Tammo en Frits, en mijn copromotor Peter bedanken. Jullie zijn een multidisciplinair team en hierdoor werd ik uitgedaagd om alle drie de 'talen' te spreken. Dit was soms een hels karwei, maar ik heb er ontzettend veel van geleerd en daar ga ik in de toekomst de vruchten van plukken. Ook wil ik jullie complimenteren voor de manier waarop jullie begeleiden. Jullie zijn toegankelijk, geven de student veel ruimte om zelf creatief te zijn en manuscripten worden altijd kritisch bestudeerd. Ik mag van geluk spreken met jullie als begeleidingsteam. Tammo, ik waardeer je gedrevenheid in het jezelf eigen maken van biomechanica. Het is knap hoe gemakkelijk jij vele PhD trajecten begeleidt met uiteenlopende onderwerpen. Frits, jij hebt ervoor gezorgd dat ik mijn weg heb kunnen vinden in de wereld van CRT. Ik vind het bijzonder om te zien hoe groot jou netwerk is en hoe zeer mensen jou waarderen in hetgeen je doet. Peter, jou diepgaande kennis over continuümmechanica heeft ervoor gezorgd dat ik altijd kritisch naar mijn resultaten bleef kijken en hierdoor zijn sommige foutjes gelukkig altijd boven komen drijven. Zonder jou was het model nooit geworden zoals het nu is.

De leden van de beoordelingscommissie, prof. Uli Schotten, prof. HansPeter Brunner-La Rocca, prof. Ralf Peeters, prof. Nico Pijls en prof. Frans van de Vosse, wil ik bedanken voor het beoordelen van het proefschrift op zijn wetenshappelijke waarde. Prof. Schotten, bedankt voor het vervullen van de voorzittersrol. De overige leden en prof. Chris Evelo dank ik voor het deelnemen aan de oppositie. I would also like to thank dr. Steve Niederer for participating in my public defense.

Bij BMT Maastricht heb ik het erg naar mijn zin gehad. Daarom wil ik mijn collega's Bart (paranimf), Jeire, Alex, Wouter D., Georgina, en Lauren ontzettend bedanken voor de leuke tijd op het lab. Wat zijn we toch eigenlijk 
een raar clubje: iedereen doet zulk verschillend onderzoek. En toch konden we mekaar helpen. Naast de PhD's wil ik ook Joost, John, Koen, Wouter H., Arnold, Theo, Claire en Jeroen bedanken.

Inmiddels zijn er ook collega's vertrokken bij BMT die ik graag nog even noem. Yvette en Nico, mijn treinbuddies destijds, en Alessandro, Jort en Evelien, ook met jullie was het de eerste jaren goed toeven bij BMT. De laatste, maar eigenlijk de belangrijkste, die ik in dit rijtje wil noemen is Wilco. Dit werk is tot stand gekomen met ontzettend veel hulp, kennis en inzicht van jou. Ik bewonder het gemak waarmee jij allerlei programmaatjes in elkaar draaide. Je was altijd bereid om mij te helpen als ik weer eens ergens niet uitkwam. Het was een hele uitdaging voor mij om op zelfstandige voet verder te gaan toen jij in eerste instantie naar Lugano vertrok. Die uitdaging werd alleen maar groter toen je besloot de wetenschap te verlaten. Het is gelukt, met dit proefschrift als resultaat. Heel erg bedankt voor al je hulp in dit traject.

Mijn collega's zaten niet alleen bij de afdeling BMT in Maastricht, maar ook bij BMT Eindhoven en met name de PhD studenten in 4.14. Het initiatief om ongeveer eens per maand met 4.14 te gaan eten was echt een goed idee. Ik zag jullie ten slotte maar 1 dag per week en op deze manier heb ik jullie allemaal beter leren kennen. De stafleden van BMT Eindhoven wil ik ook bedanken. Met name Frans bedankt voor mijn nul-aanstelling, hierdoor kon ik ook van de leuke uitjes en het goede kerstpakket van de TU/e profiteren. Ook heb ik een aantal afstudeerders van de TU/e begeleid. Adrián, Loes en Marloes heel erg bedankt voor jullie inzet. Loes, jou werk sprong er wel echt bovenuit en een deel ervan is in hoofdstuk 7 terecht gekomen.

In het laatste jaar van mijn promotie-traject heb ik de mensen bij Fysiologie Maastricht beter leren kennen. Het vroege opstaan op woensdag om bij de werkbespreking te kunnen zijn, was het altijd meer dan waard. Jullie fysiologische (Frans, Lars, Elien) en klinische (Kevin, Masih) inzichten waren altijd meer dan welkom.

Dan is er ook een groep mensen die niet zo zeer een bijdrage hebben geleverd aan de wetenschappelijke inhoud van dit proefschrift, maar die wel een hele belangrijke rol spelen in mijn leven buiten de universiteit. Allereerst mijn vriendinnen van de middelbare school Manouk, Karlijn, Marthe, Laurie, Melissa, Evelyn en Bregje. Wat is het heerlijk om met elkaar een weekendje weg te gaan en gek te doen. Ookal is een aantal van ons al moeder (in spé), ik hoop dat we nog eens een vakantie zoals in Rome gaan meemaken. Gelukkig heb ik ook vriendinnen wat dichter bij huis. Elise, ik waardeer je attentheid en het is heerlijk om samen te genieten van het leven. Laten we ervoor zorgen dat we in ons 'nieuwe leven' ook nog wat plaats houden voor ons 'oude leven'. Steffie, Anne en Marije, ook met jullie is het altijd gezellig en ik vind dat we gezellige uitjes maken. Rianne, ik vind het super leuk om weer met 
jou te roeien. En natuurlijk ook de rest van mijn roeiploeg bedankt voor alle ontspanning, competitie en gezelligheid buiten het werkende leven.

Pap en mam, bedankt dat jullie er altijd zijn en mij altijd vrij hebben gelaten in de keuzes die ik heb gemaakt. Niek en Romanée, we wonen ver uit elkaar en we zien mekaar dan niet heel vaak, maar toch is er een goede klik. Ik vind het leuk dat jullie allebei een belangrijke rol hebben in deze mijlpaal. Niek jij als paranimf en Romanée heel erg bedankt voor het ontwerpen van de kaft. Han en An, het is altijd heerlijk genieten van jullie kookkunsten. Bedankt dat jullie zo geïnteresseerd zijn geweest in mijn werk.

Als laatste en allerbelangrijkste, mijn kersverse echtgenoot Teun. Wat hebben we het heerlijk samen en wat zijn we een bijzonder jaar aan het beleven. Van het ene geluk in het ander, de ene mijlpaal na de ander. Het was hard werken om dit proefschrift af te hebben voor onze bruiloft en jij hebt me daar ontzettend veel bij gesteund. Ik kijk ernaar uit om samen nog vele mooie reizen te maken, lekker samen te sporten, te eten, te drinken, kortom, te genieten van het leven. 
About the author 


\section{Curriculum Vitae}

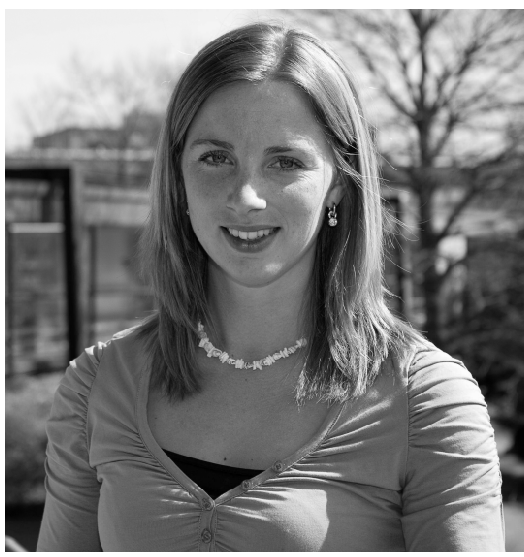

Marieke Pluijmert was born on the 3rd of September 1985 in Woerden, The Netherlands. She attended secondary education at the Minkema College in Woerden. After obtaining her atheneum (pre-university) diploma, she started her academic education in 2004 at the Eindhoven University of Technology. In April 2011 she obtained her degree Master of Science in Medical Engineering. The Master's program included an internship at the Bioengineering Institute of the University of Auckland in New Zealand (September-December 2009) under the supervision of prof. Alistair A. Young and assoc. prof. Brett Cowan. She worked on the detection of myocardial hypertrophy in the electrocardiogram. In her final Masters project, she investigated the effect of remodeling of myofiber orientations on cardiac function in the normal and situs inversus totalis left ventricle. This project was under supervision of assis. prof. Peter H.M. Bovendeerd and prof. Tammo Delhaas. From May 2011 until May 2015, she worked as a PhD candidate at Cardiovascular Research Institute Maastricht (CARIM) of the Maastricht University at the Departments of Biomedical Engineering and Physiology under supervision of prof. Tammo Delhaas and prof. Frits W. Prinzen. Assis. prof. Peter H.M. Bovendeerd was co-supervisor in this project. During her PhD project, she developed a biventricular computational model using the finite element (FE) method and applied this FE model for Cardiac Resynchronization Therapy (CRT). As of May 2015, she is working as a postdoctoral research tellow at the Departments of Physiology and Biomedical Engineering at the Maastricht University. 


\section{List of publications}

M. Pluijmert, F.W. Prinzen, A. Flores de la Parra, W. Kroon, T. Delhaas, P. Bovendeerd, How to choose myofiber orientation in a biventricular finite element model?, In: Functional Imaging and Modeling of the Heart. Ed. by H. van Assen, P. Bovendeerd, T. Delhaas, Vol. 9126, Lecture Notes in Computer Science, Springer Berlin Heidelberg, pp 373-381, 2015

M. Pluijmert, J. Lumens, M. Potse, T. Delhaas, A. Auricchio, F.W. Prinzen, Computer Modelling for Better Diagnosis and Therapy of Patients by Cardiac Resynchronisation Therapy, Arrhythmia \& Electrophysiology Review, 4(1):62-7, 2015

A.C. Rossi, M. Pluijmert, P.H.M. Bovendeerd, W. Kroon, T. Arts, T. Delhaas, Assessment and comparison of left ventricular shear in normal and situs inversus totalis hearts by means of magnetic resonance tagging, American Journal of Physiology - Heart and Circulatory Physiology, 308(5):H416-H423, 2014

M. Pluijmert, P.H.M. Bovendeerd, W. Kroon, F.W. Prinzen, T. Delhaas, Effects of Activation Pattern and Active Stress Development on Myocardial Shear in a Model with Adaptive Myofiber Reorientation, American Journal of Physiology - Heart and Circulatory Physiology, 306(4):H538-H546, 2014

M. Pluijmert, P. Bovendeerd, W. Kroon, T. Delhaas, The Effect of Active CrossFiber Stress on Shear-Induced Myofiber Reorientation, In: Functional Imaging and Modeling of the Heart. Ed. by S. Ourselin, D. Rueckert, N. Smith, Vol. 7945, Lecture Notes in Computer Science, Springer Berlin Heidelberg, pp 3545,2013

M. Pluijmert, W. Kroon, A.C. Rossi, P.H.M. Bovendeerd, T. Delhaas, Why SIT Works: Normal Function Despite Typical Myofiber Pattern in Situs Inversus Totalis (SIT) Hearts Derived by Shear-induced Myofiber Reorientation, PLoS Computational Biology, 8(7):e1002611, 2012

M. Pluijmert, W. Kroon, T. Delhaas, P.H.M. Bovendeerd, Adaptive reorientation of cardiac myofibers: the long-term effect of initial and boundary conditions, Mechanics Research Communications, 42:60-67, 2012 